\title{
B.R.N.O. CONTRIBUTIONS \#41 - TIMES OF MINIMA
}

LehkÝ, M. ${ }^{4,5}$, Hoňková, K. ${ }^{1}$; Šmelcer, L. ${ }^{1,3}$; Souza de Joode, M. ${ }^{36 *}$; BíleK ${ }^{6}$, F.; Mašek, M. ${ }^{1,7}$; Urbaník, M. ${ }^{8}$; Walter, F. ${ }^{1,9}$; Dienstbier, V. ${ }^{1,2}$; Bragagnolo, U. ${ }^{10}$; Nosál', P. ${ }^{11}$; Červinka, L. ${ }^{12}$; MazAnec, J. ${ }^{13}$; VRAŠŤÁK, M. ${ }^{14}$; Lomoz, F. ${ }^{15}$; HAnŽL, D. ${ }^{16}$; SERGEy, I. ${ }^{17}$; Gudmundsson, S. ${ }^{18}$; Jacobsen, J. ${ }^{19}$; Ehrenberger, R. ${ }^{20}$; Hladík, B. ${ }^{21}$; MAgris, M. ${ }^{22}$; Tylšar, M. ${ }^{23}$; Persha, G. ${ }^{24}$; Š́olník, V. ${ }^{25}$; Smolka, M. ${ }^{26}$; Audejean, M. ${ }^{27}$; Trnka, J. ${ }^{28}$; Medulka, T. ${ }^{29}$; Šuchañ, J. ${ }^{30}$; Salvaggio, F. ${ }^{31}$; Papini, R. ${ }^{31}$; Marchini, A. ${ }^{32}$; Colaco, C. ${ }^{32}$; Vala, J. ${ }^{436}$; Starck, M. ${ }^{32}$; Quiñones, C. ${ }^{32}$; Auer, R. F. ${ }^{33}$; Melia, R. ${ }^{34}$; Ruocco, N. ${ }^{35}$; Kalášek, J. ${ }^{9}$; Jíra, S. ${ }^{36}$; Versari, L. ${ }^{37}$; Girardici, C. ${ }^{32}$; Tornatore, M. ${ }^{32}$; Boková, S. ${ }^{9}$; Malinak, J. ${ }^{9}$; Novotný, P. ${ }^{9}$; Mokrý, A. ${ }^{9}$; Banfi, M. ${ }^{38}$; Castillo, M. ${ }^{35}$; Durantini, L. ${ }^{32}$; Hrádek, L. ${ }^{9}$; Kubica, T. ${ }^{39}$; Lamberská, I. ${ }^{36}$; López, O. ${ }^{40}$; Lyachová, K. ${ }^{9}$; MrŇÁK, P. ${ }^{1,36}$; Pavlíková, E. ${ }^{9}$; Vilchis, E. ${ }^{35}$; ZaČAl, M. ${ }^{9}$

1) Variable Star and Exoplanet Section of the Czech Astronomical Society, Fričova 298, CZ-251 65 Ondřejov, Czech Republic

2) Astronomical Institute, The Czech Academy of Sciences, Fričova 298, CZ-251 65 Ondřejov, Czech Republic

3) Valašské Meziříčí Observatory, Vsetínská 78, CZ-757 01 Valašské Meziříčí, Czech Republic

4) Astronomical Institute, Faculty of Mathematics and Physics, Charles University in Prague, V Holešovičkách 2, CZ-180 00 Prague, Czech Republic

5) Hvězdárna v Úpici, U Lipek 160, CZ-542 32 Úpice, Czech Republic

6) TS Observatory, Trocnovská 1188, CZ-374 01 Trhové Sviny, Czech Republic

7) FZU - Institute of Physics of the Czech Academy of Sciences, Na Slovance 1999/2, CZ-182 21, Praha, Czech Republic

8) Kysuce Observatory, Dolinský potok 1278, SK-024 01 Kysucké Nové Mesto, Slovak Republic

9) Štefánik Observatory, Strahovská 205, CZ-118 00 Prague, Czech Republic

10) Via Belludi, 3 - 35012 Camposampiero (PD), Italy

11) Zvolenská 129/4, SK-962 02 Vígl'aš, Slovak Republic

12) Svojsíkova 1370, Mladá Boleslav, Czech Republic

13) Hvězdárna Vyškov, Kroměřížská 721, CZ-682 01 Vyškov, Czech Republic

14) Kl'účiny 457, 03401 Liptovská Štiavnica, Slovak Republic

15) Švermova 441, CZ-264 01 Sedlčany, Czech Republic

16) Úvoz 118, CZ-602 00 Brno, Czech Republic

17) Polyani, Molodehno, Belarus

18) Nesjar Observatory, Hraunholl 5, 781 Hofn in Hornafjordur, Island

19) Egeskov Observatory, Syrenvej 6, 7000 Fredericia, Denmark

20) Vranová 183, CZ-679 62 Křetín, Czech Republic

21) Borečkova 1422, Praha 9, CZ-198 00, Czech Republic

22) Ostello scout Alpe Adria, Circolo Culturale Astrofili Trieste, Trieste, Italy

23) Hvězdárna Prostějov, Riegrova 3348, CZ-796 01 Prostějov, Czech Republic

24) Optec, Lowell, Michigan, USA

25) Broumov NMO, Hesseliova 343, CZ-550 01 Broumov, Czech Republic

26) Staničná 597, Trenčianska Turná SK-913 21, Slovak Republic

27) Astronomie en Chinonais Mairie, F-375 00, Chinon, France

28) City Observatory Slaný, Nosačická 1713, CZ-274 01 Slaný, Czech Republic

29) Vihorlatská hvězdáreň, Mierová 4, 06001 Humenné, Slovak Republic

30) Kraskova 12, Polomka, SK-976 66, Slovak Republic

Corresponding author, marco.souzadejoode@gmail.com 
31) WBRO (K49) - San Casciano in Val di Pesa (FI) - Italy

32) Observatorio Astronómico Córdoba, Laprida 854, Córdoba, Argentina

33) South-Moravian-Observatory, CZ-664 71 Chudčice 273, Czech Republic

34) UTRGV - Brownsville - Texas USA

35) Osservatorio Astronomico "Nastro Verde", MPC C82, Via Nastro Verde, Sorrento, Napoli, Italy

36) Astronomical expedition in Upice Observatory, U Lipek 160, CZ-542 32 Úpice, Czech Republic

37) A25 Nova Milanese Observatory, Via Battisti 26, Nova Milanese, Milano, Italy

38) Osservatorio Astronomico "Liceo Iris Versari", Felizzano, Alessandria, Italy

39) Planetárium Ostrava, K Planetáriu 502, CZ-725 26 Ostrava-Krásné Pole, Czech Republic

40) Cabudare, Edo Lara, Venezuela

\begin{abstract}
This paper presents 2109 times of minima for 965 objects acquired by 59 members and cooperating observers of the Variable Star and Exoplanet Section of the Czech Astronomical Society (B.R.N.O. Observing project). These observations were submitted to the website of the Variable Star and Exoplanet Section of the Czech Astronomical Society between November 2016 and March 2018.
\end{abstract}

\title{
1 Introduction
}

We introduce 2109 times of minima of 965 eclipsing binary objects. There are 1873 CCDbased observations, 217 DSLR-based and 19 observations were obtained with the use of a photoelectric photometer. These timings were uploaded to the website of the Variable Star and Exoplanet Section of the Czech Astronomical Society via the B.R.N.O. online protocols by 59 observers from around the world during 2016 - 2018. Observers are members and collaborators of the Variable Star and Exoplanet Section of the Czech Astronomical Society (VSES) - B.R.N.O. project ("Brno Regional Network of Observers" group; hereafter B.R.N.O.).

This paper contains only new, previously unpublished observations.

CCD and DSLR frames were mostly reduced by C-MuniPack code (Motl, 2007), the well-known adaptation of MuniPack code (Hroch, 1998), based on DaoPhot routines (Stetson, 1987, 1991). Specifically, the observers used the C-MuniPack versions from 2.1.13 (released in 2016) to 2.1.24 (released in 2018).

The times of minima were determined by the method which uses phenomenological description of the eclipse shape (Mikulášek, 2015; Brát et al., 2012; Cagaš \& Pejcha, 2012; Chrastina et al., 2014). There is an on-line fitting tool implemented to minima protocols in the var2.astro.cz. The uncertainty of the minimum time (one sigma significance level, column 2 in Table 1), was derived using bootstrap method (Brát et al., 2012).

All observations were first submitted to the on-line protocols of B.R.N.O. project* This submission is done by observers themselves. Subsequently, each observation is visually validated by the database administrators (K. Hoňková, L. Šmelcer, V. Dienstbier) and only observations with a well defined minimum are accepted to the on-line database.

\footnotetext{
${ }^{*}$ http://var2.astro.cz/EN/brno/protokoly.php
} 
Before publication, each minimum is marked as primary or secondary, according to the $\mathrm{O}-\mathrm{C}$ gateway $^{\dagger}$ (Paschke \& Brát, 2006). If the minimum is not included in the $\mathrm{O}-\mathrm{C}$ gateway, it is manually processed by the administrator.

\section{Description of the paper}

Table 1 contains the times of minima in HJD based on UTC. Each published observation can be viewed in detail following the link under HJD, where both the light curve and the reference frame are available, and notes made by observers can be found there.

We would like to point out that the character of a minimum (primary/secondary) depends only on used light ephemeris - especially for EW binaries. All light ephemeris used for character of minima determination were taken from the $\mathrm{O}-\mathrm{C}$ gateway. If the ephemeris were not available from the $\mathrm{O}-\mathrm{C}$ gateway, they were determined manually by the administrator on an individual basis using period analysis.

The 5th column in the Table 1 contains an independent indicator of the timing determination accuracy - the light curve minimum coverage factor - given in fractional form as a ratio between all acquired data points and data points covering decreasing branch of a minimum.

Table 2 contains list of observers with their statistics.

\section{Legend}

- Table 1 - Times of minima of eclipsing binaries (p. 5-180)

- Table 2 - List of observers (p. 181-182)

\section{Description of the Table 1}

- Column 0 - objects designation.

- Column 1 - HJD of observed minimum (JDhel - 2400 000) based on coordinated universal time UTC. Five decimal places in HJD are needed for functionality of the hyperlinks.

- Column 2 - uncertainty of minimum time determination in days.

- Column 3 - identification of primary (I) or secondary (II) minimum.

- Column 4 - used detector type and photometric band.

- Column 5 - total number of measurements/number of data on decreasing branch of light curve.

- Column 6 - observer's identification.

- Column 7 - equipment used for observation.

\footnotetext{
${ }^{\dagger}$ http://var2.astro.cz/ocgate/index.php?lang $=$ en
} 
Table 1: Times of minima of eclipsing binaries

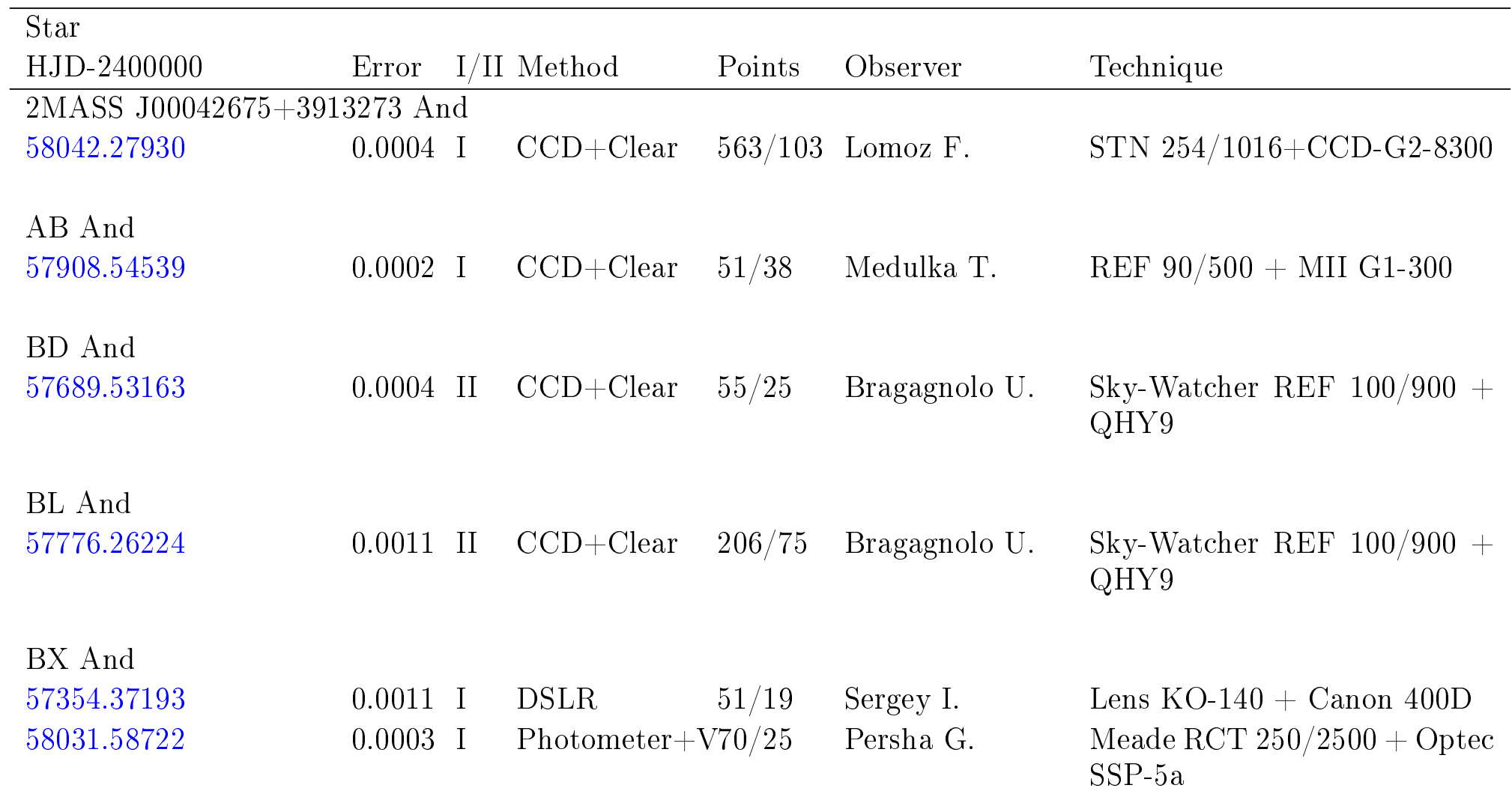

CN And

58043.42255

$0.0001 \mathrm{I} \quad \mathrm{CCD}+\mathrm{R} \quad 350 / 126$ Šmelcer $\mathrm{L}$.

Sky-Watcher NWT 254/1200 + MII G2-402

CZ And

58004.56090

$0.0003 \mathrm{I} \quad \mathrm{CCD}+\mathrm{R} \quad 94 / 50 \quad$ Vrašták M

NWT 355/1600+MII G2-1600, pointer $80 / 400+$ G1-0301

58004.56108

$0.0003 \mathrm{I} \quad \mathrm{CCD}+\mathrm{V} \quad 92 / 48 \quad$ Vrašták $\mathrm{M}$

NWT 355/1600+MII G2-1600, 80/400+G1-0301

DO And

57981.53803

0.0003 II $\quad \mathrm{CCD}+\mathrm{V} \quad$ 84/51 Vrašták M.

NWT 355/1600+MII G2-1600, 57981.53837

0.0003 II $\mathrm{CCD}+\mathrm{R} \quad 87 / 51 \quad$ Vrašták M. $80 / 400+$ G1-0301

NWT 355/1600+MII G2-1600, $80 / 400+$ G1-0301

EP And 58024.59177

0.0001 I CCD + Clear $\quad 149 / 53 \quad$ Bragagnolo U.

Sky-Watcher REF 100/900 + QHY9

GSC $\quad 02781-00387$

And 


\begin{tabular}{|c|c|c|c|c|c|c|}
\hline $\begin{array}{l}\text { Star } \\
\text { HJD-2400000 }\end{array}$ & Error & & Method & Points & Observer & Technique \\
\hline $\begin{array}{l}\text { GSC } \quad 02837-01343 \\
\text { And }\end{array}$ & & & & & & \\
\hline 57690.54456 & 0.0003 & I & CCD + Clear & $142 / 69$ & Bragagnolo U. & $\begin{array}{l}\text { Sky-Watcher REF } 100 / 900+ \\
\text { QHY9 }\end{array}$ \\
\hline GZ And & & & & & & \\
\hline 58053.55541 & 0.0002 & I & $\mathrm{CCD}+$ Clear & $174 / 35$ & Bragagnolo U. & $\begin{array}{l}\text { Sky-Watcher REF } 100 / 900+ \\
\text { QHY9 }\end{array}$ \\
\hline $\begin{array}{l}\text { KN And } \\
57714.32268\end{array}$ & 0.0004 & I & CCD+Clear & $123 / 68$ & Urbaník M. & $\begin{array}{l}\text { Sky-Watcher NWT 150/750 + } \\
\text { MII G1-0300 }\end{array}$ \\
\hline LO And & & & & & & \\
\hline 57644.38606 & 0.0001 & II & $\mathrm{CCD}+$ Clear & $171 / 132$ & Cervinka L. & R102/500 + Atik 314L + \\
\hline 58035.48029 & 0.0002 & II & CCD +Clear & $227 / 105$ & Bragagnolo U. & $\begin{array}{l}\text { Sky-Watcher REF 100/900 + } \\
\text { QHY9 }\end{array}$ \\
\hline 58091.40491 & 0.0001 & II & $\mathrm{CCD}+$ Clear & $285 / 151$ & Bragagnolo U. & $\begin{array}{l}\text { Sky-Watcher REF 100/900 + } \\
\text { QHY9 }\end{array}$ \\
\hline
\end{tabular}

NSVS 3962974 And 57707.37493

57776.32367

NSVS 6195117 And 57776.31676

NSVS 9001093 And 57984.51611

0.0004 I DSLR

187/121 Nosál' P.

TYC 2781-2154-1 And 57706.29114 58042.42126

0.0003 I $\quad C C D+$ Clear $680 / 183$ Lomoz F. 0.0005 I $\mathrm{CCD}+$ Clear 558/426 Lomoz F.

V0469 And 57981.57583 57981.57587 V0483 And 58043.47286
0.0004 I $\quad C C D+$ Clear 54/47 Urbaník M. 0.0003 I CCD+Clear 158/126 Urbaník M.

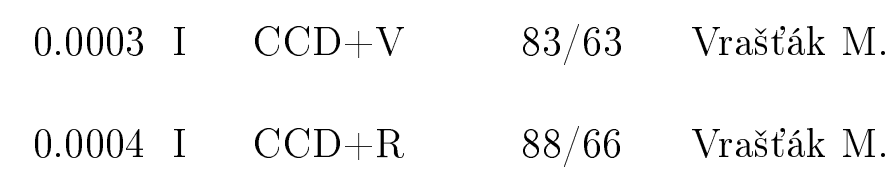

Sky-Watcher REF ED 80/600, red $0.85+$ MII G2-830 Sky-Watcher REF 80/520 + MII G2-8300

Sky-Watcher REF 100/900 + QHY9

NWT 150/600 + Canon 20D + MPCC mk.III

STN 254/1016+G2-8300

STN 254/1016+CCD-G2-8300
NWT 355/1600+MII G2-1600, 80/400+G1-0301

NWT 355/1600+MII G2-1600, 80/400+G1-0301 
Star

H.JD-2400000

Error I/II Method

Points Observer

Technique

V0488 And

57973.54566

$0.0005 \mathrm{I} \quad \mathrm{CCD}+\mathrm{R} \quad$ 154/93 Lehky M.

HK25, NWT 250/1000 + SBIG ST-7 + BVRcIc

V0489 And 57332.32951

V0512 And 57980.54769

57980.54940

57996.57279

V0518 And 58025.50719

V0523 And 58064.36444

V0527 And 57783.36689

58026.49567

58094.45570

V0530 And 57781.35653

V0546 And 57658.32787

57753.31442

57776.29530
0.0005 II $\quad$ CCD + Clear $92 / 50 \quad$ Ehrenberger R.

$0.0003 \mathrm{I} \quad \mathrm{CCD}+\mathrm{R} \quad 49 / 30 \quad$ Lehky $\mathrm{M}$.

$0.0006 \mathrm{I} \quad \mathrm{CCD}+\mathrm{V} \quad 46 / 29 \quad$ Lehky $\mathrm{M}$.

0.0008 II $\quad \mathrm{CCD}+\mathrm{R} \quad 151 / 114$ Lehky $\mathrm{M}$.

0.0003 I CCD $+\mathrm{R} \quad 130 / 48$ Hanžl D.

0.0002 I DSLR $\quad 169 / 54$ Nosál P.

0.0007 I CCD + Clear 69/52 Urbaník M.

$0.0002 \mathrm{I} \quad \mathrm{CCD}+$ Clear $405 / 292$ Walter F.

0.0003 I CCD + Clear 397/283 Bragagnolo U.

0.0005 I CCD + Clear 304/226 Urbaník M.

0.0002 I CCD + Clear $\quad 145 / 38$ Jacobsen J.

0.0002 I CCD + Clear 189/110 Urbaník M.

0.0002 I CCD + Clear 158/84 Urbaník M.
Quadruplet LNS 65/420+MII G2-8300

HK25, NWT 250/1000 + SBIG ST-7 + BVRcIc

HK25, NWT 250/1000 + SBIG ST-7 + BVRcIc HK25, NWT 250/1000 + SBIG ST-7 + BVRcIc

DATEL, NWT 200/800 + MII G2 8300

NWT $150 / 600+$ Canon 20D + MPCC mk.III

Sky-Watcher NWT 150/750 + MII G2-8300

$\mathrm{CZ}$ Kometensucher REF $200 / 1370$ + SBIG S7

Sky-Watcher REF 100/900 + QHY9

Sky-Watcher NWT 150/750 + MII G2-8300

EQ6, 80 mm refractor, SXVRH16

Sky-Watcher REF 80/520 + MII G2-8300

Sky-Watcher REF 80/520 + MII G2-8300 


\begin{tabular}{|c|c|c|c|c|c|}
\hline Star & & & & & \\
\hline HJD-2400000 & Error & I/II Method & Points & Observer & Technique \\
\hline 57700.43394 & 0.0014 & I $\quad$ DSLR & $183 / 84$ & Nosál' P. & $\begin{array}{l}\text { NWT 150/600 + Canon 20D + } \\
\text { MPCC mk.III }\end{array}$ \\
\hline 58041.38002 & 0.0005 & CCD + Clear & $90 / 44$ & Ehrenberger R. & NWT 150/600+MII G2-8300 \\
\hline 58042.35898 & 0.0007 & CCD + Clear & $119 / 43$ & Ehrenberger R. & NWT 150/600+MII G2-8300 \\
\hline 58077.37107 & 0.0003 & $\mathrm{CCD}+$ Clear & $103 / 42$ & $\begin{array}{l}\text { Gudmundsson } \\
\text { S. }\end{array}$ & $\begin{array}{l}400 \mathrm{~mm} \text { LX200 SCT/SBIG } \\
\text { STL11k CCD }\end{array}$ \\
\hline
\end{tabular}

V0566 And

58068.49622

58071.41943

58072.39305

58074.34552

V0579 And 57984.51407

V0600 And 58004.40728

58004.40745

V0625 And 57924.48409

57924.48433

V0683 And 57971.48249

V0725 And 57995.37571

57995.37597

57995.37667
$0.0003 \mathrm{I} \quad \mathrm{CCD}+\mathrm{V} \quad 93 / 74$

$0.0001 \mathrm{I} \quad \mathrm{CCD}+\mathrm{V} \quad 65 / 54$

$0.0004 \mathrm{I} \quad \mathrm{CCD}+\mathrm{V}$

$0.0002 \mathrm{I} \quad \mathrm{CCD}+\mathrm{V}$

0.0006 II DSLR

0.0002 II $\mathrm{CCD}+\mathrm{V}$

40/12 Vrašták M

0.0002 II $\mathrm{CCD}+\mathrm{R}$

45/17 Vrašták M
Gudmundsson $\mathrm{S}$.

Gudmundsson S.

$71 / 12$

$116 / 33$

Gudmundsson $\mathrm{S}$.

Gudmundsson $\mathrm{S}$.

186/119 Nosál P.
$400 \mathrm{~mm}$ LX200 SCT/SBIG STL11k CCD

$400 \mathrm{~mm}$ LX200 SCT/SBIG STL11k CCD

$400 \mathrm{~mm}$ LX200 SCT/SBIG STL11k CCD

$400 \mathrm{~mm}$ LX200 SCT/SBIG STL11k CCD

NWT $150 / 600+$ Canon 20D + MPCC mk.III

NWT 355/1600+MII G2-1600, 80/400+G1-0301

NWT 355/1600+MII G2-1600, 80/400+G1-0301

NWT 355/1600+MII G2-1600, 80/400+G1-0301

NWT 355/1600+MII G2-1600, 80/400+G1-0301

Sky-Watcher NWT 250/1200 + Algol 0402

NWT 355/1600+MII G2-1600, 80/400+G1-0301

NWT 355/1600+MII G2-1600, 80/400+G1-0301

NWT 355/1600+MII G2-1600, 80/400+G1-0301 


\begin{tabular}{|c|c|c|c|c|c|}
\hline Star & & & & & \\
\hline HJD-2400000 & Error & I/II Method & Points & Observer & Technique \\
\hline 57725.21290 & 0.0005 & I $\quad$ DSLR & $116 / 17$ & Nosál' P. & $\begin{array}{l}\text { NWT } 150 / 600+\text { Canon 20D + } \\
\text { MPCC mk.III }\end{array}$ \\
\hline 57725.34445 & 0.0004 & DSLR & $116 / 106$ & Nosál' P. & $\begin{array}{l}\text { NWT } 150 / 600+\text { Canon } 20 \mathrm{D}+ \\
\text { MPCC mk.III }\end{array}$ \\
\hline
\end{tabular}

VSX J235743.9+361213 And

$\begin{array}{lllllll}58057.27807 & 0.0001 & \text { I } & \text { CCD }+ \text { Clear } & 240 / 59 & \text { Lomoz F. } & \text { STN 254/1016+CCD-G2-8300 } \\ \text { WZ And } & & & & & & \\ 57692.32243 & 0.0001 & \text { II } & \text { CCD }+ \text { Clear } & 250 / 124 & \text { Červinka L. } & \text { R102/500+ Atik 314L+ }+ \\ 57732.32358 & 0.0002 & \text { I } & \text { CCD }+ \text { Clear } & 71 / 51 \quad \text { Banfi M. } & \begin{array}{l}\text { MEADE LX200R, 25cm f } / 5, \\ \text { CCD SBIG ST7E NABG }\end{array} \\ 58064.50708 & 0.001 & \text { II } & \text { DSLR } & 180 / 156 & \text { Nosál P. } & \begin{array}{l}\text { NWT 150/600 + Canon 20D + } \\ \text { MPCC mk.III }\end{array}\end{array}$

ASAS J101302-2925.2 Ant

$\begin{array}{lllllll}57759.80668 & 0.0004 \text { I } & \text { CCD }+ \text { R } & 64 / 52 & \text { Mašek M. } & \begin{array}{l}\text { FRAM, Nikkor LNS 106/300 } \\ + \text { G4-16000 }\end{array} \\ 57759.80670 & 0.0005 \text { I } & \text { CCD }+ \text { Clear } & 64 / 52 & \text { Mašek M. } & \begin{array}{l}\text { FRAM, Nikkor LNS 106/300 } \\ + \text { G4-16000 }\end{array}\end{array}$

ASAS J200326+1448.5 Aql

57925.46969

0.0004 I CCD+Clear 194/132 Urbaník M.

Sky-Watcher NWT 150/750 + MII G2-8300

GDS J1842057-024311 Aql

57930.53251

0.0012 I $\quad$ CCD + Clear $\quad 54 / 34$

Salvaggio

F., $\quad$ MK-C $12 \mathrm{f} / 5.6+$ GM-2000 + Marchini

A., STL-6303

Papini R.

GSC 05165-00276 Aql

57659.32557

0.0002 I CCD + Clear $368 / 177$ Lomoz F.

SNT 254/1016+G2-8300

OO Aql

57995.41550

$0.0001 \mathrm{I} \quad \mathrm{CCD}+\mathrm{V} \quad$ 295/126 Šmelcer L.

Sky-Watcher NWT 254/1200

57995.41550

$0.0001 \mathrm{I} \quad \mathrm{CCD}+\mathrm{R} \quad 284 / 121 \quad$ Šmelcer L.

+ MII G2-402

Sky-Watcher NWT 254/1200

+ MII G2-402

SvkV081 Aql

57892.51768

0.0003 II $\quad \mathrm{CCD}+\mathrm{V} \quad 52 / 22 \quad$ Smolka M.

NWT $250 / 1000+$ ATIK $314 \mathrm{~L}++$ MPCC 


\begin{tabular}{|c|c|c|c|c|c|}
\hline \multicolumn{6}{|l|}{ Star } \\
\hline HJD-2400000 & Error & I/II Method & Points & Observer & Technique \\
\hline $\begin{array}{l}\text { V0346 Aql } \\
58012.63349\end{array}$ & 0.0001 & Photom & V56/33 & Persha G. & Meade RCT $250 / 2500+$ Optec \\
\hline
\end{tabular}

V0479 Aql 57975.42643

V0609 Aql 57934.45917

V0879 Aql 57975.37155

57975.50663

V1096 Aql

57934.43176

V1355 Aql

57939.39789

V1692 Aql

57968.43340
0.0002 I CCD +Clear 273/126 Urbaník M.

0.0003 I CCD+Clear 124/67 Urbaník M.

0.0001 II CCD+Clear 269/46 Urbaník M.

0.0004 I CCD+Clear 269/234 Urbaník M.

0.0008 I CCD+Clear 148/61 Urbaník M.

0.0001 I CCD+Clear 238/78 Urbaník M.

0.0014 I CCD +Clear 186/128 Nosál P.
Sky-Watcher NWT 150/750 + MII G2-8300

Sky-Watcher NWT 150/750 + MII G2-8300

Sky-Watcher NWT 150/750 + MII G2-8300

Sky-Watcher NWT 150/750 + MII G2-8300

Sky-Watcher NWT 150/750 + MII G2-8300

Sky-Watcher NWT 150/750 + MII G2-8300

REF $102 / 500+$ ATIK 16 IC mono

STN 254/1016+CCD-G2-8300

STN 254/1016+CCD-G2-8300

DD Aqr 57995.53360

0.0002 I $\quad$ CCD + Clear $416 / 123$ Červinka L.
STN 254/1016+CCD-G2-8300

0.0003 I $\quad \mathrm{CCD}+\mathrm{R} \quad 473 / 351$ Lomoz F.

R102/500 + Atik 320E mono, $0.5 \mathrm{x} F$ 


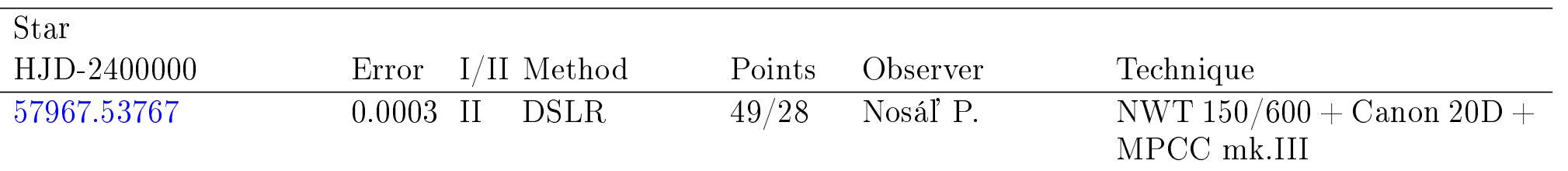

V0338 Aqr 57984.45158

V0350 Aqr 57986.44801

V0351 Aqr 57995.60523

57995.60558

KO Ara 57892.76925
0.0001 I CCD+Clear 208/122 Nosál' P.

0.0004 I CCD +Clear 198/90 Nosál' P.

0.0008 II CCD + Clear 209/174 Červinka L.

0.001 II CCD + Clear 406/345 Červinka L.
REF 102/600 + ATIK 16 IC mono

REF $102 / 500+$ ATIK 16 IC mono

R102/500 + Atik 320E mono, $0.5 \mathrm{x}$ FC

R102/500 + Atik 320E mono, $0.5 \mathrm{x} \mathrm{FC}$

BQ Ari

58043.46523

0.0001 I $\quad$ CCD + Clear $\quad 99 / 45$

Girardini

C., Meade LX200 SC + CCD

Colazo

C.,

Starck M.

58043.60764

0.0008 I DSLR 266/88 Školník V.

Tamron LNS 44/300 + Canon 600D

266/227 Školník V.

Tamron LNS 44/300 + Canon 600D

CSS J030933.9+300228 Ari

57659.46441

0.0006 I $\quad C C D+$ Clear 359/102 Lomoz F.

SNT 254/1016+G2-8300

CSS J030950.6+302419 Ari

57659.52546

0.0004 I CCD + Clear 337/147 Lomoz F.

STN 254/1016+G2-8300

CSS J031204.2+302609 Ari

57659.57243

0.0003 I CCD + Clear 285/240 Lomoz F.

STN 254/1016+G2-8300

HAT 259-0010571 Ari

58080.36489

0.0006 I $\mathrm{CCD}+$ Clear $\quad 92 / 16 \quad$ Mašek M.

NWT 150/600 + CCD MII G2-1600 


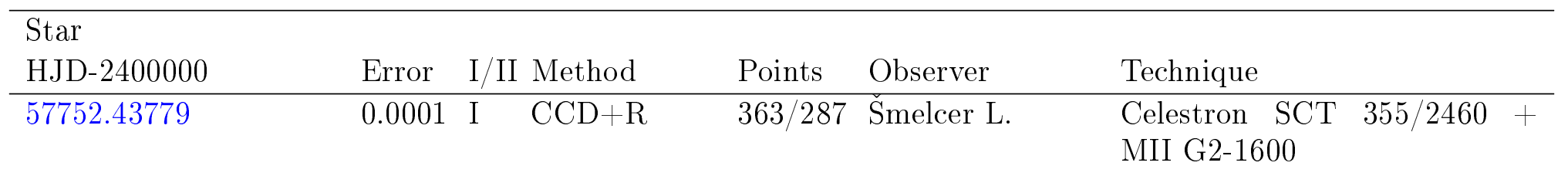

RX Ari

57996.53297

57996.53318

57996.53358

SS Ari

57646.49049

57720.78446

SZ Ari

57727.44404

57727.45330

58080.39354

AH Aur

57762.32721

57828.28988

AP Aur

58091.69244

58122.44668

CG Aur

57720.49340

57720.49347

57720.49398
0.0002 I $\quad \mathrm{CCD}+\mathrm{B} \quad 52 / 12 \quad$ Vrašták M.

0.0002 I $\quad \mathrm{CCD}+\mathrm{V} \quad 40 / 14 \quad$ Vrašták M.

0.0003 I $\quad \mathrm{CCD}+\mathrm{R} \quad 56 / 15 \quad$ Vrašták M.

0.0001 I CCD + Clear 551/229 Červinka L.

$0 \quad$ I $\quad \mathrm{CCD}+$ Clear

1117/779 Melia R., Colazo C., Vilchis

E.

$\begin{array}{lllll}0.0027 & \text { II } & \text { CCD }+\mathrm{I} & 21 / 9 & \text { Mašek M. } \\ 0.0015 & \text { II } & \text { CCD }+ \text { R } & 20 / 10 & \text { Mašek M. } \\ 0.0003 \text { I } & \text { CCD +Clear } & 64 / 32 & \text { Mašek M. }\end{array}$

NWT 150/600 + CCD MII G2-1600 + comacorector NWT 150/600 + CCD MII G2-1600 + comacorector NWT 150/600 + CCD MII G2-1600

NWT 355/1600+MII G2-1600, 80/400+G1-0301

NWT 355/1600+MII G2-1600, $80 / 400+$ G1-0301

NWT 355/1600+MII G2-1600, 80/400+G1-0301

$\mathrm{R} 102 / 500+$ Atik $314 \mathrm{~L}+$

Telesc $16+\mathrm{CCD}$
Sky-Watcher NWT 250/1200 + Algol 0402

Sky-Watcher NWT 254/1200 + MII G2-402 


\begin{tabular}{|c|c|c|c|c|c|}
\hline Star & & & & & \\
\hline HJD-2400000 & Error & I/II Method & Points & Observer & Technique \\
\hline 57739.38488 & 0.0002 & I $\quad \mathrm{CCD}+$ Clear & $92 / 35$ & Šmelcer L. & $\begin{array}{l}\text { Celestron SCT } 280 / 1765+ \\
\text { MII G2-4000 }\end{array}$ \\
\hline 57739.38531 & 0.0003 & $\mathrm{CCD}+\mathrm{R}$ & $85 / 32$ & Šmelcer L. & $\begin{array}{l}\text { Celestron SCT } 280 / 1765+ \\
\text { MII G2-4000 }\end{array}$ \\
\hline
\end{tabular}

CSS J070856.3+353755 Aur

$\begin{array}{llllll}57776.55244 & 0.0008 & \text { I } & \text { DSLR } & 80 / 12 & \text { Walter F. } \\ 57776.55396 & 0.0003 & \text { I } & \text { CCD }+ \text { R } & 175 / 24 & \text { Walter F. } \\ 57776.55406 & 0.0002 & \text { I } & \text { CCD }+ \text { Clear } & 179 / 27 & \text { Walter F. } \\ 57776.55442 & 0.0001 & \text { I } & \text { CCD }+ \text { I } & 170 / 25 & \text { Walter F. }\end{array}$

CzeV1066 Aur 57722.34157 0.0005 II DSLR

159/100 Červinka L.

Skywatcher REF 102/600, Canon $450 \mathrm{~d}$

MARK SCT 406/4060, SBIG ST10XME

MARK SCT 406/4060, SBIG ST10XME

MARK SCT 406/4060, SBIG ST10XME

DN Aur 57722.36970 0.0025 I DSLR

147/113 Červinka L.

R102/500 + Canon 1000D

EM Aur 57735.25470 57773.51525 57773.51582 58112.40619

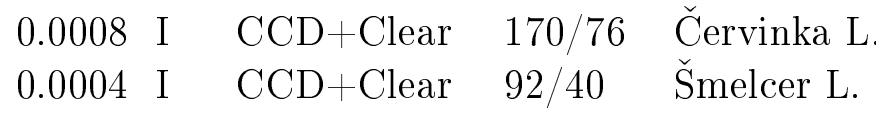
R102/500 + Atik 314L + Celestron SCT 280/1765 + MII G2-4000 0.0005 I $\quad \mathrm{CCD}+\mathrm{R} \quad 89 / 40 \quad \check{\text { Śmelcer } \mathrm{L} .}$ Celestron SCT 280/1765 MII G2-4000 $0.0006 \mathrm{I} \quad \mathrm{CCD}+\mathrm{V} \quad 154 / 83 \quad$ Magris M. Achromatic refractor 120/600, CCD Orion Starshoo

EP Aur 57776.43753 0.0002 I CCD+Clear 139/60 Urbaník M. Sky-Watcher REF 80/520 + MII G2-8300

HL Aur 58080.41656 58080.41656 58080.41679 58080.41711

$\begin{array}{lllll}0.0002 & \text { II } & \text { CCD }+ \text { V } & 32 / 17 & \text { Lehky M. } \\ 0.0002 & \text { II } & \text { CCD }+ \text { I } & 33 / 16 & \text { Lehky M. } \\ 0.0002 & \text { II } & \text { CCD }+ \text { R } & 33 / 17 & \text { Lehky M. } \\ 0.0003 & \text { II } & \text { CCD }+ \text { B } & 31 / 16 & \text { Lehky M. }\end{array}$
JST, NWT $400 / 2000+$ MII G2-1600 + BVRcIc JST, NWT 400/2000 + MII G2-1600 + BVRcIc JST, NWT 400/2000 + MII G2-1600 + BVRcIc JST, NWT 400/2000 + MII G2-1600 + BVRcIc 


\begin{tabular}{|c|c|c|c|c|c|}
\hline $\begin{array}{l}\text { Star } \\
\text { HJD-2400000 }\end{array}$ & Error & I/II Method & Points & Observer & Technique \\
\hline HW Aur & & & & & \\
\hline 58186.30208 & 0.0003 & $\mathrm{CCD}+$ Clear & 199/101 & Urbaník M. & $\begin{array}{l}\text { Sky-Watcher NWT 150/750 + } \\
\text { MII G2-8300 }\end{array}$ \\
\hline
\end{tabular}

IM Aur 58203.32364

IU Aur 57800.38495

KU Aur 57751.36480

NN Aur 57632.53002

NSVS 4312408 Aur 57799.34526

NSVS 6859986 Aur 57722.34066

NSVS 6868895 Aur 58117.30842

NSVS 7006595 Aur 57781.33033

NSVS 7217296 Aur 57776.57860

SERIV 21 Aur 57799.32837

SERIV 22 Aur 57799.28161 SERIV 23 Aur 57799.34588 57752.39090

0.0001 I CCD +Clear 558/259 Urbaník M.

0.0006 II CCD + Clear 208/100 Červinka L. $0.0002 \mathrm{I} \quad \mathrm{CCD}+\mathrm{R} \quad 1170 / 555$ Šmelcer L.

0.0009 II $\mathrm{CCD}+$ Clear $360 / 227$ Červinka L.

0.0001 I CCD +Clear 130/63 Medulka T.

0.0013 I DSLR

0.0002 I $\quad$ CCD + Clear 504/321 Červinka L.

0.0007 I $\quad C C D+$ Clear $\quad 374 / 182$ Červinka L.

0.0003 I CCD + Clear $\quad 788 / 536$ Červinka L.

R102/500 + Atik 314L+

0.0022 I DSLR 64/22 Walter F.

0.0003 I DSLR

88/32 Sergey I.

Lens KO-140 + Canon 400D

SvkV034 Aur
0.0034 I DSLR

104/39 Sergey I.

Lens Jupiter-21 + Canon 400D
0.0015 I DSLR 115/81 I.Sergey Lens Jupiter-21 + Canon 400D

Sky-Watcher REF $80 / 520+$ MII G2-8300

$\mathrm{R} 102 / 500+$ Atik $314 \mathrm{~L}+$ Sky-Watcher NWT 254/1200 + MII G2-402

$\mathrm{R} 102 / 500+$ Atik $314 \mathrm{~L}+$

REF 90/500 + MII G1-300

Lens Jupiter-21 + Canon 400D

Tamron 55-200@135mm, Atik $320 \mathrm{E}$ mono

Skywatcher REF 102/600, Canon $450 \mathrm{~d}$ 


\begin{tabular}{|c|c|c|c|c|c|c|}
\hline $\begin{array}{l}\text { Star } \\
\text { HJD-2400000 }\end{array}$ & Error & $\mathrm{I} / \mathrm{Il}$ & Method & Points & Observer & Technique \\
\hline 56008.32292 & 0.0005 & II & $\mathrm{CCD}+$ Clear & $34 / 15$ & Urbaník M. & $\begin{array}{l}\text { Sky-Watcher REF } 80 / 520+ \\
\text { MII G1-0300 }\end{array}$ \\
\hline 56009.30796 & 0.0003 & II & CCD + Clear & $37 / 15$ & Urbaník M. & $\begin{array}{l}\text { Sky-Watcher REF } 80 / 520+ \\
\text { MII G1-0300 }\end{array}$ \\
\hline 56010.28939 & 0.0009 & II & CCD + Clear & $62 / 12$ & Urbaník M. & $\begin{array}{l}\text { Sky-Watcher REF } 80 / 520+ \\
\text { MII G1-0300 }\end{array}$ \\
\hline 56011.39520 & 0.0006 & I & $\mathrm{CCD}+$ Clear & $92 / 68$ & Urbaník M. & $\begin{array}{l}\text { Sky-Watcher NWT 150/750 + } \\
\text { MII G2-8300 }\end{array}$ \\
\hline 56269.47669 & 0.0003 & II & CCD + Clear & $123 / 88$ & Urbaník M. & $\begin{array}{l}\text { Sky-Watcher SCT 102/1200 + } \\
\text { MII G1-0300 }\end{array}$ \\
\hline 56271.32182 & 0.0009 & I & $\mathrm{CCD}+$ Clear & $69 / 32$ & Urbaník M. & $\begin{array}{l}\text { Sky-Watcher SCT 102/1200+ } \\
\text { MII G1-0300 }\end{array}$ \\
\hline 57728.41818 & 0.0003 & I & $\mathrm{CCD}+$ Clear & $98 / 75$ & Urbaník M. & $\begin{array}{l}\text { Sky-Watcher REF } 80 / 520+ \\
\text { MII G1-0300 }\end{array}$ \\
\hline 57772.27172 & 0.0004 & II & CCD + Clear & $65 / 28$ & Urbaník M. & $\begin{array}{l}\text { Sky-Watcher REF } 80 / 520+ \\
\text { MII G2-8300 }\end{array}$ \\
\hline 57799.41898 & 0.0003 & I & CCD + Clear & $110 / 26$ & Urbaník M. & $\begin{array}{l}\text { Sky-Watcher NWT 150/750 + } \\
\text { MII G2-8300 }\end{array}$ \\
\hline 58113.26982 & 0.0004 & II & $\mathrm{CCD}+$ Clear & $136 / 98$ & Urbaník M. & $\begin{array}{l}\text { Sky-Watcher NWT 150/750 + } \\
\text { MII G2-8300 }\end{array}$ \\
\hline 58134.27448 & 0.0002 & I & CCD + Clear & $158 / 91$ & Urbaník M. & $\begin{array}{l}\text { Sky-Watcher NWT 150/750 + } \\
\text { MII G2-8300 }\end{array}$ \\
\hline
\end{tabular}

T Aur

58041.41492

$0.0005 \mathrm{I} \quad \mathrm{CCD}+\mathrm{R}$

137/17 Mazanec J.

ORION， NWT 500/1500 + MII G2 402

58041.41543

$0.0011 \mathrm{I} \quad \mathrm{CCD}+\mathrm{V}$

114/14 Mazanec J.

ORION, NWT 500/1500 + MII G2 402

UCAC2 42376345 Aur 58041.48563

$0.0003 \mathrm{I} \quad \mathrm{CCD}+\mathrm{R}$

129/79 Mazanec J.

ORION, NWT 500/1500 MII G2 402

58041.48614

0.0003 I $\quad \mathrm{CCD}+\mathrm{I}$

131/76 Mazanec J.

ORION， NWT 500/1500 + MII G2 402

58041.48621

0.0004 I $\quad \mathrm{CCD}+\mathrm{V}$

132/76 Mazanec J.

ORION, NWT 500/1500 + MII G2 402

V0364 Aur

58117.27511

0.0006 I $\quad \mathrm{CCD}+\mathrm{V}$

155/74 Magris M.

REF $120 / 600+$ Orion 3G $\mathrm{CCD}+0.5 \mathrm{x}$ reducer

V0404 Aur

57722.42004

$0.0002 \mathrm{I} \quad \mathrm{CCD}+\mathrm{V}$

41/21 Lehky M.

JST, NWT 400/2000 + MII

G2-1600 + BVRcIc

57722.42039

$0.0002 \mathrm{I} \quad \mathrm{CCD}+\mathrm{R} \quad 39 / 20 \quad$ Lehky M.

JST, NWT $400 / 2000+$ MII

G2-1600 + BVRcIc 
Star

HJD-2400000

Error I/II Method

Points Observer

Technique

V0417 Aur 58117.28958

V0449 Aur 57829.43521

V0459 Aur 57755.33676

V0495 Aur 57841.39495

57841.39613

V0534 Aur 57762.29442 57777.28390

V0535 Aur 58186.38916

58186.39010

58186.39096

V0591 Aur 57628.50980 57727.26992

V0607 Aur 58175.29273 58179.42685
0.0003 I CCD + Clear $226 / 124$ Červinka L.

0.0004 I DSLR

0.0006 I $\quad C C D+V \quad 46 / 15 \quad \check{\text { Suchaň J. }}$

$0.0003 \mathrm{I} \quad \mathrm{CCD}+\mathrm{V} \quad 158 / 81 \quad \check{\text { Šmelcer L }}$.

$0.0002 \mathrm{I} \quad \mathrm{CCD}+\mathrm{R} \quad 177 / 92 \quad$ Šmelcer L.
Tamron 55-200@135mm, Atik 320E mono

Lens Jupiter-21 + Canon 400D

Sky-Watcher NWT 250/1200 + Algol 0402

Sky-Watcher NWT 254/1200 + MII G2-402

Sky-Watcher NWT 254/1200 + MII G2-402

Sky-Watcher NWT 250/1200 + Algol 0402

Sky-Watcher REF 80/520 + MII G2-8300

ORION, NWT 500/1500 + MII G2 402

ORION, NWT 500/1500 + MII G2 402

ORION, NWT 500/1500 + MII G2 402

REF 90/500 + MII G1-300

Sky-Watcher REF 80/520 + MII G2-8300

Sky-Watcher NWT 150/750+ MII G2-8300

Sky-Watcher NWT 150/750 + MII G2-8300 


\begin{tabular}{|c|c|c|c|c|c|}
\hline \multicolumn{6}{|l|}{ Star } \\
\hline HJD-2400000 & Error & I/II Method & Points & Observer & Technique \\
\hline 58149.38098 & 0.0001 & I $\quad \mathrm{CCD}+\mathrm{I}$ & $99 / 60$ & Mazanec J. & $\begin{array}{l}\text { ORION, NWT } 500 / 1500 \\
\text { MII G2 } 402\end{array}$ \\
\hline 58149.38120 & 0.0001 & $\mathrm{CCD}+\mathrm{V}$ & $95 / 56$ & Mazanec J. & $\begin{array}{l}\text { ORION, NWT } 500 / 1500+ \\
\text { MII G2 } 402\end{array}$ \\
\hline 58149.38173 & 0.0001 & $\mathrm{CCD}+\mathrm{R}$ & $102 / 63$ & Mazanec J. & $\begin{array}{l}\text { ORION, NWT } 500 / 1500+ \\
\text { MII G2 } 402\end{array}$ \\
\hline
\end{tabular}

V0620 Aur 57740.23725

V0623 Aur 57826.36984

57829.38553

V0636 Aur 57798.29727 57799.31987

V0640 Aur 58043.53069 58080.43258

V0641 Aur 57713.39898

57753.28410

57753.28413

57753.28428

V0803 Aur 57776.41646

ZZ Aur

57720.34449 57735.37459
0.001 I CCD +Clear 144/48 Urbaník M.

0.0006 I CCD + Clear 141/80 Bragagnolo U.

0.0005 I CCD + Clear $\quad 116 / 78$ Bragagnolo U.

Sky-Watcher REF 100/900 + QHY9

Sky-Watcher REF 100/900 QHY9

0.0006 I CCD + Clear $431 / 276$ Červinka L.

0.0006 I $\mathrm{CCD}+$ Clear $407 / 328$ Červinka L.

$\mathrm{R} 102 / 500+$ Atik 314L +

$\mathrm{R} 102 / 500+$ Atik $314 \mathrm{~L}+$

$\begin{array}{llll}0.0005 \text { I } & \text { CCD }+ \text { R } & 157 / 93 & \text { Lehky M. } \\ 0.0004 \text { II } & \text { CCD }+ \text { R } & 104 / 62 & \text { Lehky M. }\end{array}$

HK25， NWT 250/1000 + SBIG ST-7 + BVRcIC HK25, NWT 250/1000 + SBIG ST-7 + Rc

0.0001 I CCD + Clear 128/51 Urbaník M.

$0.0001 \mathrm{I} \quad \mathrm{CCD}+\mathrm{R} \quad 195 / 119$ Mazanec J.

$0.0001 \mathrm{I} \quad \mathrm{CCD}+\mathrm{V} \quad 203 / 124$ Mazanec J.

$0.0001 \mathrm{I} \quad \mathrm{CCD}+\mathrm{I} \quad 202 / 124$ Mazanec J.

Sky-Watcher NWT 150/750 + MII G2-8300

ORION, NWT 500/1500 + MII G2 402

ORION, NWT 500/1500 + MII G2 402

ORION, NWT 500/1500 + MII G2 402

Sky-Watcher REF 80/520 +

0.0007 I CCD +Clear 138/38 Urbaník M.

\section{G2-8300}

$\mathrm{R} 102 / 500+$ Atik $314 \mathrm{~L}+$

0.0004 II CCD + Clear 220/137 Červinka L.

Sky-Watcher REF 80/520 + MII G2-8300

Sky-Watcher REF 80/520 MII G2-8300 


\begin{tabular}{|c|c|c|c|c|c|c|}
\hline Star & & & & & & \\
\hline HJD-2400000 & Error & $\mathrm{I} / \mathrm{I}$ & Method & Points & Observer & Technique \\
\hline 58093.69765 & 0.0004 & II & $\mathrm{CCD}+$ Clear & $173 / 93$ & Bragagnolo U. & $\begin{array}{l}\text { Sky-Watcher REF } 100 / 900+ \\
\text { QHY9 }\end{array}$ \\
\hline 58113.23959 & 0.0001 & I & $\mathrm{CCD}+$ Clear & $129 / 62$ & Urbaník M. & $\begin{array}{l}\text { Sky-Watcher NWT 150/750 + } \\
\text { MII G2-8300 }\end{array}$ \\
\hline 58134.28246 & 0.0001 & I & $\mathrm{CCD}+$ Clear & $148 / 94$ & Urbaník M. & $\begin{array}{l}\text { Sky-Watcher NWT 150/750 + } \\
\text { MII G2-8300 }\end{array}$ \\
\hline
\end{tabular}

AC Boo

\begin{tabular}{|c|c|c|c|c|c|}
\hline 57841.51175 & $0.0001 \mathrm{I}$ & $\mathrm{CCD}+\mathrm{I}$ & $95 / 62$ & Vrašták M & $\begin{array}{l}\text { NWT 280/1500+MII G2-1600, } \\
80 / 400+\text { G1-0301 }\end{array}$ \\
\hline 57841.51194 & $0.0001 \mathrm{I}$ & $\mathrm{CCD}+\mathrm{R}$ & $94 / 62$ & Vrašták M & $\begin{array}{l}\text { NWT } 280 / 1500+\text { MII G2-1600, } \\
80 / 400+\text { G1-0301 }\end{array}$ \\
\hline 57841.51213 & $0.0002 \mathrm{I}$ & $\mathrm{CCD}+\mathrm{V}$ & $95 / 62$ & Vrašták M & $\begin{array}{l}\text { NWT } 280 / 1500+\text { MII G2-1600, } \\
80 / 400+\text { G1-0301 }\end{array}$ \\
\hline 57841.51235 & $0.0002 \mathrm{I}$ & $\mathrm{CCD}+\mathrm{B}$ & $95 / 62$ & Vrašták M & $\begin{array}{l}\text { NWT } 280 / 1500+\text { MII G2-1600, } \\
80 / 400+\text { G1-0301 }\end{array}$ \\
\hline 57989.36373 & $0.0002 \mathrm{I}$ & $\mathrm{CCD}+$ Clear & $226 / 136$ & Bragagnolo U. & $\begin{array}{l}\text { Sky-Watcher REF 100/900 + } \\
\text { QHY9 }\end{array}$ \\
\hline
\end{tabular}

AQ Boo

57839.41363

0.0002 II CCD + Clear 66/23 Lehky M.

HK25, NWT 250/1000

SBIG ST-7 + C

57844.57788

0.0003 I CCD + Clear 74/41 Mašek M.

NWT $150 / 600+$ CCD MII

57844.57791

$0.0005 \mathrm{I} \quad \mathrm{CCD}+\mathrm{R} \quad 96 / 29 \quad$ Lehky M.

G2-1600 + comacorector

HK25, NWT 250/1000 + SBIG ST-7 + Rc

CzeV614 Boo

57839.51046

0.0014 I DSLR 198/98 Nosál' P.

NWT 150/600 + Canon 20D + MPCC mk.III

EF Boo

57798.52043

0.0001 II CCD + Clear 478/256 Lehky M.

HK25， NWT 250/1000 + SBIG ST-7 + C

EL Boo

57892.40793

0.0009 II CCD + Clear 102/31 Hladík B.

LNS 38/135 + ATIK 320E MuniWin

EQ Boo

57853.49283

0.0009 II DSLR

228/159 Nosál' P.

REF 102/500 + Canon 20D

EW Boo 


\begin{tabular}{|c|c|c|c|c|c|c|}
\hline \multicolumn{7}{|l|}{ Star } \\
\hline HJD-2400000 & Error & I/II & Method & Points & Observer & Technique \\
\hline 57800.56652 & 0.0004 & I & $\mathrm{CCD}+\mathrm{B}$ & $82 / 48$ & Lehky M. & $\begin{array}{l}\text { JST, NWT } 400 / 2000+\text { MII } \\
\text { G2-1600 + BVRcIc }\end{array}$ \\
\hline 57800.56669 & 0.0003 & I & $\mathrm{CCD}+\mathrm{V}$ & $75 / 47$ & Lehky M. & $\begin{array}{l}\text { JST, NWT } 400 / 2000+\text { MII } \\
\text { G2-1600 + BVRcIc }\end{array}$ \\
\hline 57800.56682 & 0.0002 & I & $\mathrm{CCD}+\mathrm{I}$ & $84 / 50$ & Lehky M. & $\begin{array}{l}\text { JST, NWT } 400 / 2000+\text { MII } \\
\text { G2-1600 + BVRcIc }\end{array}$ \\
\hline \multicolumn{7}{|l|}{ GG Boo } \\
\hline 57800.60263 & 0.0003 & I & CCD + Clear & $237 / 139$ & Lehky M. & $\begin{array}{l}\text { HK25, NWT 250/1000 + } \\
\text { SBIG ST-7 + C }\end{array}$ \\
\hline \multicolumn{7}{|l|}{ GK Boo } \\
\hline 57799.64206 & 0.0001 & II & CCD + Clear & $212 / 59$ & Lehky M. & $\begin{array}{l}\text { HK25, NWT 250/1000 + } \\
\text { SBIG ST-7 + C }\end{array}$ \\
\hline 57825.44125 & 0.0001 & II & $\mathrm{CCD}+$ Clear & $445 / 186$ & Červinka L. & $\mathrm{R} 102 / 500+$ Atik $314 \mathrm{~L}+$ \\
\hline 57853.39060 & 0.0001 & I & CCD + Clear & $283 / 164$ & Urbaník M. & $\begin{array}{l}\text { Sky-Watcher NWT 150/750 + } \\
\text { MII G2-8300 }\end{array}$ \\
\hline 57853.39089 & 0.0001 & I & CCD + Clear & $83 / 62$ & $\begin{array}{l}\text { Mašek M., Tyl- } \\
\text { šar M. }\end{array}$ & $\begin{array}{l}\text { NWT } 250 / 1000+\text { CCD MII } \\
\text { G2-8300 }\end{array}$ \\
\hline
\end{tabular}

GM Boo

57878.45581

GN Boo

57905.41465

57905.41498

57905.41543

GR Boo

57876.39012

57876.39013

57876.39056

57892.39807

57892.39844

57892.39870
0.0003 II CCD+Clear 472/337 Vraštták M.

39/21 Lehky M.

0.0003 I $\quad \mathrm{CCD}+\mathrm{I}$

0.0002 I $\quad \mathrm{CCD}+\mathrm{R}$

$0.0004 \mathrm{I} \quad \mathrm{CCD}+\mathrm{V}$

37/19 Lehky M.

37/20 Lehky M.
NWT 150/750+G1-0301

HK25, NWT 250/1000 SBIG ST-7 + BVRcIc HK25, NWT 250/1000 SBIG ST-7 + BVRcIc HK25, NWT 250/1000 SBIG ST-7 + BVRcIc

HK25, NWT 250/1000 SBIG ST-7 + BVRcIc HK25, NWT 250/1000 SBIG ST-7 + BVRcIc HK25, NWT 250/1000 SBIG ST-7 + BVRcIc HK25, NWT 250/1000 SBIG ST-7 + BVRcIc HK25, NWT 250/1000 SBIG ST-7 + BVRcIC HK25, NWT 250/1000 SBIG ST-7 + BVRcIc 


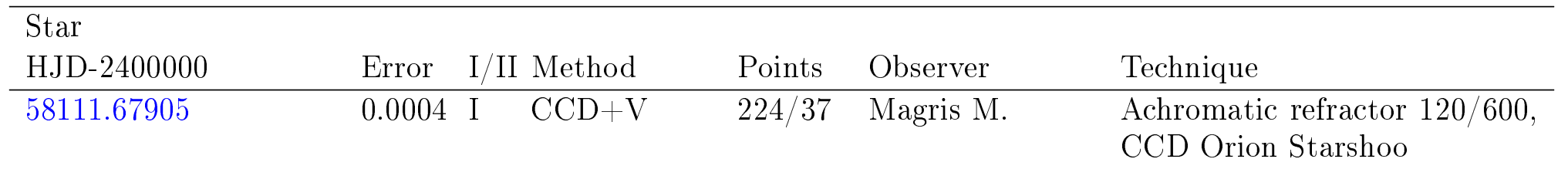

GU Boo

57868.34820

57868.34823

57868.59347

57868.59362

57929.43906

57929.43934

57929.43967

57941.41505

57948.49982

57954.36488

GV Boo

57839.52626

57844.48729

HH Boo

57844.36730

58163.51374

I Boo

57906.63004

IL Boo

57800.62231

57800.62246

57839.35838
$0.0001 \mathrm{I} \quad \mathrm{CCD}+\mathrm{R} \quad 167 / 24 \quad$ Šmelcer L.

$0.0002 \mathrm{I} \quad \mathrm{CCD}+\mathrm{V} \quad 159 / 22 \quad$ Śmelcer $\mathrm{L}$.

0.0001 II $\quad \mathrm{CCD}+\mathrm{R} \quad 65 / 51 \quad$ Lehky M.

0.0001 II $\quad \mathrm{CCD}+\mathrm{V} \quad 63 / 50 \quad$ Lehky M.

$0.0001 \mathrm{I} \quad \mathrm{CCD}+\mathrm{V} \quad 211 / 137$ Šmelcer L.

$0.0001 \mathrm{I} \quad \mathrm{CCD}+\mathrm{V} \quad 84 / 29 \quad$ Lehky M.

$0.0001 \mathrm{I} \quad \mathrm{CCD}+\mathrm{R} \quad 88 / 30 \quad$ Lehky M.

0.0004 II $\quad \mathrm{CCD}+\mathrm{V} \quad 143 / 97 \quad$ Šmelcer L.

0.0001 I CCD +V 195/145 Šmelcer L.

0.0001 I $\quad C C D+V \quad 254 / 38 \quad$ Šmelcer L.

0.0003 II CCD + Clear 112/65 Lehky M.

$0.0003 \mathrm{I} \quad \mathrm{CCD}+\mathrm{R} \quad 125 / 57 \quad$ Lehky M.

0.0001 I CCD + Clear 149/57 Červinka L.

0.0003 II CCD + Clear 63/48 Walter

Pavlíková

Mašek M.

F., CZ Kometensucher REF

E., $\quad 200 / 1370+$ CCD SBIG ST7

HK25， NWT 250/1000 + SBIG ST-7 + C

HK25, NWT 250/1000 + SBIG ST-7 + Rc

Sky-Watcher NWT 254/1200

G2-402

+ MII G2-402

JST, NWT 400/2000 + MII

G2-1600 + BVRcIc

JST, NWT 400/2000 + MII

G2-1600 + BVRcIc

Sky-Watcher NWT 254/1200

+ MII G2-402

JST, NWT 400/2000 + MII

G2-1600 + BVRcIc

JST, NWT 400/2000 + MII

G2-1600 + BVRcIc

Sky-Watcher NWT 254/1200

+ MII G2-402

Sky-Watcher NWT 254/1200

+ MII G2-402

Sky-Watcher NWT 254/1200

+ MII G2-402 


\section{Star \\ IO Boo}

HJD-2400000

57864.37385

57864.37469

57864.37482

57864.37502

57864.50768

57864.50781

57864.50856

57864.50861

IX Boo

57844.49736

KK Boo

57829.48290

57905.40754

57905.40801

57905.54847

57905.54921

KP Boo

57904.50528
Error I/II Method

Points Observer

86/16 Mazanec J.

95/16 Mazanec J.

0.0002 II $\mathrm{CCD}+\mathrm{R}$

0.0002 II $\mathrm{CCD}+\mathrm{I}$

84/14 Mazanec J.

0.0002 II $\mathrm{CCD}+\mathrm{V}$

0.0003 I $\mathrm{CCD}+\mathrm{R}$

0.0005 I $\mathrm{CCD}+\mathrm{B}$

0.0004 I $\quad \mathrm{CCD}+\mathrm{I}$

0.0005 I $\quad \mathrm{CCD}+\mathrm{V}$

$83 / 70$

Mazanec J.

83/16 Mazanec J.

95/76 Mazanec J.

86/75 Mazanec J.

84/71 Mazanec J.
Technique

ORION, NWT 500/1500 +

MII G2 402

ORION, NWT 500/1500 +

MII G2 402

ORION, NWT 500/1500 +

MII G2 402

ORION, NWT 500/1500 +

MII G2 402

ORION, NWT 500/1500 MII G2 402

ORION, NWT 500/1500 +

MII G2 402

ORION, NWT 500/1500 + MII G2 402

ORION, NWT 500/1500+ MII G2 402

NWT $150 / 600+$ Canon 20D + MPCC mk.III

MARK SCT 406/4060, SBIG ST10XME

JST, NWT 400/2000 + MII G2-1600 + BVRcIc

JST, NWT $400 / 2000+$ MII G2-1600 + BVRcIc

JST, NWT 400/2000 + MII G2-1600 + BVRcIc

JST, NWT 400/2000 + MII G2-1600 + BVRcIc

\section{LINEAR 13643051}

Boo

57840.54030

57841.46090

0.0003 I CCD + Clear $\quad 355 / 200$ Bragagnolo U.

Sky-Watcher REF 100/900 + QHY9

0.0007 I CCD + Clear $\quad$ 173/110 Urbaník M.

0.0008 I CCD + Clear 223/48 Urbaník M.
Sky-Watcher NWT 150/750 + MII G2-8300

Sky-Watcher NWT 150/750 + MII G2-8300 


\begin{tabular}{llllllll}
\hline Star & & & & & & \\
HJD-2400000 & Error & I/II Method & Points & Observer & Technique & \\
\hline 57825.50355 & 0.0004 & I & CCD + Clear & $323 / 83$ & Smolka M. & NWT 250/1000 & + ATIK \\
& & & & & & $314 \mathrm{~L}++$ ACC &
\end{tabular}

LINEAR 14714767

Boo

57839.43446

57839.56899

MN Boo

57829.45873

NSVS 2800224 Boo

57841.45217

57844.53580

NSVS 5168364 Boo

57839.42058

57839.59179

NX Boo

57837.49305

57837.49317

57837.49348

57837.49371

57846.53569

NY Boo

57447.30978

OP Boo

57471.49530
0.0011 I DSLR

0.0014 II DSLR

0.0008 I DSLR

0.0003 I CCD+Clear 233/37 Urbaník M.

0.0002 I CCD +Clear 280/147 Urbaník M.

0.0005 II DSLR 198/33 Nosál' P.

0.0006 I DSLR

0.0003 II $\quad \mathrm{CCD}+\mathrm{V} \quad 137 / 74 \quad$ Mazanec J.

0.0002 II $\quad \mathrm{CCD}+\mathrm{R} \quad 139 / 76 \quad$ Mazanec J.

0.0004 II $\quad \mathrm{CCD}+\mathrm{B} \quad 129 / 71 \quad$ Mazanec J.

0.0004 II $\quad \mathrm{CCD}+\mathrm{I}$

0.0004 II DSLR

0.0003 I CCD + Clear $\quad 210 / 73 \quad$ Jacobsen J.
NWT 150/600 + Canon 20D + MPCC mk.III

NWT 150/600 + Canon 20D + MPCC mk.III
198/157 Nosál P.

Sky-Watcher NWT 150/750 + MII G2-8300

Sky-Watcher NWT 150/750 + MII G2-8300

NWT 150/600 + Canon 20D + MPCC mk.III

NWT 150/600 + Canon 20D + MPCC mk.III

ORION, NWT 500/1500 + MII G2 402

ORION, NWT 500/1500 + MII G2 402

ORION, NWT 500/1500 + MII G2 402

138/75 Mazanec J.

110/32 Nosál P.
ORION, NWT 500/1500 + MII G2 402

NWT 150/600 + Canon 20D + MPCC mk.III

\section{OQ Boo}




\begin{tabular}{|c|c|c|c|c|c|}
\hline Star & & & & & \\
\hline HJD-2400000 & Error & I/II Method & Points & Observer & Technique \\
\hline 57888.39163 & 0.0007 & II $\mathrm{CCD}+$ Clear & $336 / 44$ & Audejean M. & $\begin{array}{l}0.32-\mathrm{m} \mathrm{f} / 6 \text { Newtonian reflector } \\
+\mathrm{CCD}\end{array}$ \\
\hline 57917.44941 & 0.0003 & $\mathrm{CCD}+$ Clear & $397 / 197$ & Audejean M. & $\begin{array}{l}0.32-\mathrm{m} \mathrm{f} / 6 \text { Newtonian reflector } \\
+\mathrm{CCD}\end{array}$ \\
\hline
\end{tabular}

OS Boo

57876.43388

57892.42708

57892.42722

57892.42937

PS Boo

57878.45989

57927.45728

PT Boo

57825.56970

57893.45951

PU Boo

57907.49095

PY Boo

57827.43895

57863.44773

QQ Boo

57839.43690

57839.57559

57841.51234

57841.64699

57844.55336
0.0001 I CCD + Clear 91/51 Audejean M.

$0.0003 \mathrm{I} \quad \mathrm{CCD}+\mathrm{V} \quad 74 / 48 \quad$ Mazanec J.

$0.0002 \mathrm{I} \quad \mathrm{CCD}+\mathrm{R} \quad 79 / 47 \quad$ Mazanec J.

$0.0003 \mathrm{I} \quad \mathrm{CCD}+\mathrm{I} \quad 78 / 46 \quad$ Mazanec J. 0.32-m f/6 Newtonian reflector $+\mathrm{CCD}$

ORION， NWT 500/1500 + MII G2 402

ORION, NWT 500/1500 MII G2 402

ORION, NWT 500/1500 + MII G2 402

$$
\begin{array}{lllll}
0.0001 \text { II } & \text { CCD }+ \text { Clear } 155 / 103 \text { Audejean M. } & \begin{array}{l}
0.32-\mathrm{m} \mathrm{f} / 6 \text { Newtonian reflector } \\
+\mathrm{CCD}
\end{array} \\
0.0002 \text { II } & \text { CCD }+ \text { Clear } & 341 / 165 \text { Audejean M. } \begin{array}{l}
0.32-\mathrm{m} \mathrm{f} / 6 \text { Newtonian reflector } \\
+\mathrm{CCD}
\end{array}
\end{array}
$$

NWT $250 / 1000+$ ATIK $314 \mathrm{~L}++\mathrm{ACC}$

0.0007 I CCD +Clear 94/71 Ehrenberger R. Quadruplet LNS 65/420+MII G2-8300

Zeiss MCT 350/3300 + Canon $450 \mathrm{~d}$

$300 \mathrm{~mm}$ LX200 SCT/SBIG STL11k CCD

$0.32-\mathrm{m} \mathrm{f} / 6$ Newtonian reflector $+\mathrm{CCD}$ S.

0.0001 II CCD + Clear 98/48 Audejean M. 


\begin{tabular}{|c|c|c|c|c|c|}
\hline \multicolumn{6}{|l|}{ Star } \\
\hline HJD-2400000 & Error & $\mathrm{I} / \mathrm{II}$ & Method & Points & Observer \\
\hline 57891.41503 & 0.0002 & $\mathrm{I}$ & CCD + Clear & $114 / 62$ & Ehrenberge \\
\hline \multicolumn{6}{|l|}{ QW Boo } \\
\hline 57844.46231 & 0.0001 & II & $\mathrm{CCD}+\mathrm{R}$ & $86 / 14$ & Lehky M. \\
\hline 57844.46232 & 0.0002 & II & $\mathrm{CCD}+\mathrm{I}$ & $81 / 14$ & Lehky M. \\
\hline 57844.46252 & 0.0002 & II & $\mathrm{CCD}+\mathrm{V}$ & $80 / 14$ & Lehky M. \\
\hline 57844.60678 & 0.0002 & I & $\mathrm{CCD}+\mathrm{I}$ & $81 / 62$ & Lehky M. \\
\hline 57844.60698 & 0.0001 & I & $\mathrm{CCD}+\mathrm{V}$ & $80 / 61$ & Lehky M. \\
\hline 57844.60705 & 0.0001 & I & $\mathrm{CCD}+\mathrm{R}$ & $86 / 64$ & Lehky M. \\
\hline 57853.33212 & 0.0003 & $\mathrm{I}$ & $\mathrm{CCD}+\mathrm{I}$ & $118 / 5$ & Lehky M. \\
\hline 57853.33276 & 0.0004 & I & $\mathrm{CCD}+\mathrm{V}$ & $117 / 5$ & Lehky M. \\
\hline 57853.33330 & 0.0002 & I & $\mathrm{CCD}+\mathrm{R}$ & $111 / 7$ & Lehky M. \\
\hline 57853.47982 & 0.0002 & II & $\mathrm{CCD}+\mathrm{V}$ & $117 / 61$ & Lehky M. \\
\hline 57853.48006 & 0.0002 & II & $\mathrm{CCD}+\mathrm{R}$ & $111 / 58$ & Lehky M. \\
\hline 57853.48022 & 0.0002 & II & $\mathrm{CCD}+\mathrm{I}$ & $118 / 58$ & Lehky M. \\
\hline 57853.62346 & 0.0001 & I & $\mathrm{CCD}+\mathrm{V}$ & $117 / 112$ & Lehky M. \\
\hline 57853.62369 & 0.0002 & I & $\mathrm{CCD}+\mathrm{R}$ & $111 / 105$ & Lehky M. \\
\hline 57853.62413 & 0.0004 & $\mathrm{I}$ & $\mathrm{CCD}+\mathrm{I}$ & $118 / 112$ & Lehky M. \\
\hline 57874.42147 & 0.0002 & II & $\mathrm{CCD}+\mathrm{R}$ & $104 / 35$ & Lehky M. \\
\hline 57874.42188 & 0.0001 & II & $\mathrm{CCD}+\mathrm{V}$ & $103 / 34$ & Lehky M. \\
\hline 57874.42201 & 0.0001 & II & $\mathrm{CCD}+\mathrm{I}$ & $105 / 35$ & Lehky M. \\
\hline 57874.56667 & 0.0002 & I & $\mathrm{CCD}+\mathrm{R}$ & $105 / 89$ & Lehky M. \\
\hline 57874.56667 & 0.0002 & I & $\mathrm{CCD}+\mathrm{I}$ & $105 / 89$ & Lehky M. \\
\hline 57874.56733 & 0.0001 & I & $\mathrm{CCD}+\mathrm{V}$ & $103 / 88$ & Lehky M. \\
\hline
\end{tabular}

JST, NWT $400 / 2000+$ MII G2-1600 + BVRcIc

JST, NWT 400/2000 + MII G2-1600 + BVRcIc

JST, NWT 400/2000 + MII G2-1600 + BVRcIc

JST, NWT 400/2000 + MII G2-1600 + BVRcIc

JST, NWT 400/2000 + MII G2-1600 + BVRcIc

JST, NWT 400/2000 + MII G2-1600 + BVRcIc

JST, NWT 400/2000 + MII G2-1600 + BVRcIc

JST, NWT 400/2000 + MII G2-1600 + BVRcIc

JST, NWT $400 / 2000+$ MII G2-1600 + BVRcIc

JST, NWT 400/2000 + MII G2-1600 + BVRcIc

JST, NWT 400/2000 + MII G2-1600 + BVRcIc

JST, NWT 400/2000 + MII G2-1600 + BVRcIc

JST, NWT 400/2000 + MII G2-1600 + BVRcIc

JST, NWT 400/2000 + MII G2-1600 + BVRcIc

JST, NWT 400/2000 + MII G2-1600 + BVRcIc

JST, NWT 400/2000 + MII G2-1600 + BVRcIc

JST, NWT 400/2000 + MII G2-1600 + BVRcIc

JST, NWT 400/2000 + MII G2-1600 + BVRcIc

JST, NWT 400/2000 + MII G2-1600 + BVRcIc

JST, NWT 400/2000 + MII G2-1600 + BVRcIc

JST, NWT 400/2000 + MII G2-1600 + BVRcIc 


\begin{tabular}{|c|c|c|c|c|c|}
\hline \multicolumn{6}{|l|}{ Star } \\
\hline HJD-2400000 & Error & I/II Method & Points & Observer & Technique \\
\hline 57884.38513 & 0.0001 & I $\quad \mathrm{CCD}+\mathrm{V}$ & $107 / 56$ & Mazanec J. & $\begin{array}{l}\text { ORION, NWT } 500 / 1500+ \\
\text { MII G2 402 }\end{array}$ \\
\hline 57884.38540 & 0.0001 & $\mathrm{CCD}+\mathrm{R}$ & $109 / 59$ & Mazanec J. & $\begin{array}{l}\text { ORION, NWT } 500 / 1500+ \\
\text { MII G2 } 402\end{array}$ \\
\hline 57884.38569 & 0.0002 & $\mathrm{CCD}+\mathrm{I}$ & $106 / 56$ & Mazanec J. & $\begin{array}{l}\text { ORION, NWT } 500 / 1500 \\
\text { MII G2 } 402\end{array}$ \\
\hline
\end{tabular}

QY Boo

57860.54169

57896.43981

57908.40202

SU Boo

57800.55857

TU Boo

57840.62107

TY Boo

57798.62137

57798.62188

57798.62192

57841.43553

57846.50993

TZ Boo

57844.47709

57929.46581

V0373 Boo

57874.44807

VW Boo

57837.37246

$\begin{array}{lllll}0.0007 \text { I } & \text { DSLR } & 117 / 59 & \text { Nosál P. } & \begin{array}{l}\text { NWT 150/600 + Canon 20D + } \\ \text { MPCC mk.III }\end{array} \\ 0.0001 \text { I } & \text { CCD }+ \text { Clear } & 359 / 184 \text { Audejean M. } & \begin{array}{l}0.32-m \text { f } / 6 \text { Newtonian reflector } \\ + \text { CCD }\end{array} \\ 0.0003 \text { I } & \text { CCD }+ \text { R } & 467 / 90 & \text { Kubica T. } & \begin{array}{l}\text { Meade SCT } 300 / 3000+\text { CCD } \\ \text { SBIG ST-8 }\end{array}\end{array}$

0.002 II $\quad$ CCD + Clear $\quad 433 / 146$ Červinka L. $\quad$ R102 $/ 500+$ Atik 314L +

0.0001 II $\quad \mathrm{CCD}+\mathrm{U} \quad 182 / 90 \quad$ Bragagnolo $\mathrm{U}$.

Sky-Watcher REF 100/900 + QHY9

\begin{tabular}{|c|c|c|c|c|c|}
\hline 0.0003 & II & $\mathrm{CCD}+$ Clear & $73 / 39$ & $\begin{array}{l}\text { Walter F., Hla- } \\
\text { dík B. }\end{array}$ & $\begin{array}{l}\text { REF200/1370 + ST7 }+ \text { Mu- } \\
\text { niWin }\end{array}$ \\
\hline 0.0003 & II & DSLR & $184 / 62$ & $\begin{array}{l}\text { Walter F., Hla- } \\
\text { dík B. }\end{array}$ & $\begin{array}{l}\text { CZ Mirotar LNS 180/1000 + } \\
\text { Canon 350D + MuniWin }\end{array}$ \\
\hline 0.0003 & II & DSLR & $186 / 61$ & $\begin{array}{l}\text { Walter F., Hla- } \\
\text { dík B. }\end{array}$ & $\begin{array}{l}\text { CZ Mirotar 180/1000 + Canon } \\
350 \mathrm{D}+\text { MuniWin }\end{array}$ \\
\hline 0.0002 & II & CCD + Clear & $202 / 110$ & Vrašták M & RF 80/400+G1-0301 \\
\hline 0.0002 & II & DSLR & $113 / 14$ & Nosál' P. & $\begin{array}{l}\text { NWT } 150 / 600+\text { Canon 20D + } \\
\text { MPCC mk.III }\end{array}$ \\
\hline
\end{tabular}

0.0001 I $\quad$ CCD + Clear $505 / 131$ Červinka L. $\quad$ R102/500 + Atik 314L +

0.0003 I DSLR 76/42 Walter F., No- CZ Mirotar LNS 180/1000 + votný P., Mali- Canon 350d nak J.

0.0004 I DSLR $\quad 155 / 82 \quad$ Nosál P.

REF $102 / 500+$ Canon 20D

XY Boo 


\begin{tabular}{|c|c|c|c|c|c|}
\hline \multicolumn{6}{|l|}{ Star } \\
\hline HJD-2400000 & Error & I/II Method & Points & Observer & Technique \\
\hline 57825.58836 & 0.0003 & I $\quad \mathrm{CCD}+\mathrm{R}$ & $200 / 181$ & Šmelcer L. & $\begin{array}{l}\text { Celestron SCT } 355 / 2460+ \\
\text { MII G2-1600 }\end{array}$ \\
\hline 57828.55117 & 0.0001 & $\mathrm{CCD}+\mathrm{R}$ & $349 / 280$ & Šmelcer L. & $\begin{array}{l}\text { Sky-Watcher NWT 254/1200 } \\
+ \text { MII G2-402 }\end{array}$ \\
\hline
\end{tabular}

ASAS J042052-3945.7 Cae

$57735.73691 \quad 0.0008$ I $\quad C C D+R \quad 193 / 125$ Mašek M.

ASAS J042851-4035.3 Cae

$57735.74888 \quad 0.0008$ I $\quad$ CCD + R $\quad 193 / 139$ Mašek M.

AO Cam

57735.31290

57735.31333

57735.31351

57735.31359

AW Cam

57619.46920

CD Cam

57754.34405

CP Cam

57810.39291

58041.43882

CV Cam

57739.34477

58044.47812

58044.47940

$\begin{array}{lllll}0.0002 & \text { I } & \text { CCD }+ \text { V } & 48 / 30 & \text { Lehky M. } \\ 0.0001 & \text { I } & \text { CCD }+B & 48 / 28 & \text { Lehky M. } \\ 0.0001 & \text { I } & \text { CCD }+ \text { I } & 55 / 32 & \text { Lehky M. } \\ 0.0001 & \text { I } & \text { CCD }+ \text { R } & 47 / 29 & \text { Lehky M. }\end{array}$

0.0001 I CCD +Clear 484/240 Medulka T.

0.0004 I CCD + Clear 626/181 Magris M.

0.0002 I CCD + Clear 576/400 Bragagnolo U.

0.0004 I DSLR

290/116 Školník V.
FRAM, Nikkor LNS 106/300

+ G4- 16000

FRAM, Nikkor LNS 106/300 + G4-16000
JST, NWT 400/2000 + MII

G2-1600 + BVRcIc

JST, NWT 400/2000 + MII G2-1600 + BVRcIc

JST, NWT 400/2000 + MII

G2-1600 + BVRcIc

JST, NWT 400/2000 + MII G2-1600 + BVRcIc

REF 90/500 + MII G1-300

Achromatic refractor 120/600, CCD Orion Starshoo 
Star
HJD-2400000
CzeV1249 Cam

58026.54621

CzeV1250 Cam

58026.55383

CzeV1254 Cam

58026.55249

CzeV663 Cam

57839.43287

57839.57205

57840.41221

57840.55283

57841.39013

57841.52940

57844.32550

57844.46599

57844.60585

57853.41361

57853.55305

CzeV863 Cam

57884.37970

\section{DN Cam}

58046.36758

LR Cam

57764.42878
0.0018 I $\quad \mathrm{CCD}+\mathrm{V} \quad 433 / 347$ Červinka L.

0.0016 I $\quad \mathrm{CCD}+\mathrm{V} \quad 347 / 299$ Červinka L.

0.0019 I $\quad \mathrm{CCD}+\mathrm{V} \quad 429 / 354$ Červinka L.

0.0005 I CCD + Clear 305/125 Bílek F.

0.0005 II CCD+Clear 305/239 Bílek F.

0.0007 II CCD+Clear 330/124 Bílek F.

0.0005 I CCD +Clear 330/249 Bílek F.

0.0006 I CCD+Clear 330/112 Bílek F.

0.0007 II CCD+Clear 330/239 Bílek F.

0.0007 II CCD+Clear 340/44 Bílek F.

0.0004 I CCD +Clear 340/172 Bílek F.

0.0007 II CCD+Clear 340/303 Bílek F.

0.0009 I CCD +Clear 331/121 Bílek F.

0.0014 II CCD + Clear 331/251 Bílek F.

0.0005 I $\quad$ CCD + Clear $233 / 65 \quad$ Šmelcer L.

Celestron SCT 280/1765 + MII G2-4000

0.0003 II CCD + Clear 149/80 Vrašták M. Pentacon 1.8/50+MII G2-1600
R102/500 + Atik 320E mono, $0.5 \mathrm{x} \mathrm{FC}$

R102/500 + Atik 320E mono, $0.5 \mathrm{x}$ FC

R102/500 + Atik 320E mono, $0.5 \mathrm{x}$ FC

NWT 200/860 + CCD Atik $314 \mathrm{~L}+$

NWT 200/860 + CCD Atik $314 \mathrm{~L}+$

NWT 200/860 + CCD Atik $314 \mathrm{~L}+$

NWT 200/860 + CCD Atik $314 \mathrm{~L}+$

NWT 200/860 + CCD Atik $314 \mathrm{~L}+$

NWT 200/860 + CCD Atik $314 \mathrm{~L}+$

NWT 200/860 + CCD Atik $314 \mathrm{~L}+$

NWT 200/860 + CCD Atik $314 \mathrm{~L}+$

NWT 200/860 + CCD Atik $314 \mathrm{~L}+$

NWT 200/860 + CCD Atik $314 \mathrm{~L}+$

NWT 200/860 + CCD Atik $314 \mathrm{~L}+$ 


\begin{tabular}{llllllll}
\hline Star & & & & & & \\
HJD-2400000 & Error & I/II Method & Points & Observer & Technique & \\
\hline 57884.47053 & 0.0001 & II & CCD + Clear & $274 / 188$ & Śmelcer L. & $\begin{array}{l}\text { Celestron SCT } \\
\text { MII G2-4000 }\end{array}$ & \\
& & & & & & MII G0/1765 & +
\end{tabular}

NR Cam

57775.53351

57777.32423

57777.45206

57800.61012

NSVS 01031772 Cam 57839.38875

57839.57263

57840.30904

57840.49315

57841.41355

57841.59747

57844.35857

57844.54268

57853.37808

57853.56204

57868.47155

57884.48575

57891.48054

57892.40098

57895.34624

57895.53008

57901.42027
0.0001 I CCD + Clear 196/130 Bragagnolo U.

$0.0001 \mathrm{I} \quad \mathrm{CCD}+\mathrm{R} \quad 342 / 158$ Šmelcer L.

0.0001 II $\mathrm{CCD}+\mathrm{R} \quad 342 / 329$ Šmelcer L.

0.0001 I DSLR

302/147 Walter F.

$0 \quad$ I $\quad$ CCD + Clear 369/119 Bílek F.

0.0001 II CCD +Clear 369/291 Bílek F.

0.0001 II CCD + Clear 325/36 Bílek F.

0.0001 I $\quad$ CCD + Clear 325/190 Bílek F.

0.0001 II CCD+Clear 346/139 Bílek F.

0.0001 I CCD+Clear 346/310 Bílek F.

0.0001 II CCD+Clear 349/82 Bílek F.

0.0001 I CCD +Clear 349/254 Bílek F.

0.0001 I CCD + Clear 330/88 Bílek F.

0.0001 II CCD+Clear 330/259 Bílek F.

$0.0003 \mathrm{I} \quad \mathrm{CCD}+\mathrm{B} \quad 244 / 53 \quad$ Šmelcer L.

0.0001 II $\quad$ CCD + R 291/223 Šmelcer L.

0.0001 I CCD+Clear 244/143 Bílek F.

0.0001 I CCD+Clear 236/64 Bílek F.

0.0001 I $\quad$ CCD +Clear 236/17 Bílek F.

0.0001 II CCD+Clear 236/187 Bílek F.

0.0001 I CCD + Clear 225/77 Bílek F.
Sky-Watcher REF 100/900 + QHY9

Celestron SCT 355/2460

MII G2-1600

Celestron SCT $355 / 2460+$

MII G2-1600

CZ MCT 350/3300, Canon $350 \mathrm{~d}$

NWT 200/860 + CCD Atik $314 \mathrm{~L}+$

NWT 200/860 + CCD Atik $314 \mathrm{~L}+$

NWT 200/860 + CCD Atik $314 \mathrm{~L}+$

NWT 200/860 + CCD Atik $314 \mathrm{~L}+$

NWT 200/860 + CCD Atik $314 \mathrm{~L}+$

NWT 200/860 + CCD Atik $314 \mathrm{~L}+$

NWT 200/860 + CCD Atik $314 \mathrm{~L}+$

NWT 200/860 + CCD Atik $314 \mathrm{~L}+$

NWT 200/860 + CCD Atik $314 \mathrm{~L}+$

NWT 200/860 + CCD Atik $314 \mathrm{~L}+$

Celestron SCT 280/1765 +

MII G2-4000

Celestron SCT 355/2460 + MII G2-1600

NWT 200/860 + CCD Atik $314 \mathrm{~L}+$

NWT 200/860 + CCD Atik $314 \mathrm{~L}+$

NWT 200/860 + CCD Atik $314 \mathrm{~L}+$

NWT 200/860 + CCD Atik $314 \mathrm{~L}+$

NWT 200/860 + CCD Atik $314 \mathrm{~L}+$ 


\begin{tabular}{|c|c|c|c|c|c|c|}
\hline \\
\hline \multirow{2}{*}{$\begin{array}{l}\text { HJD-2400000 } \\
57902.34045\end{array}$} & \multirow{2}{*}{$\begin{array}{l}\text { Error } \\
0.0001\end{array}$} & \multicolumn{2}{|c|}{ I/II Method } & \multirow{2}{*}{$\begin{array}{l}\text { Points } \\
280 / 7\end{array}$} & \multirow{2}{*}{$\begin{array}{l}\text { Observer } \\
\text { Śmelcer L. }\end{array}$} & \multirow{2}{*}{$\begin{array}{l}\text { Technique } \\
\text { Celestron SCT } 280 / 1765 \quad+ \\
\text { MII G2-4000 }\end{array}$} \\
\hline & & $\mathrm{I}$ & CCD + Clear & & & \\
\hline 57902.52407 & 0.0001 & II & CCD + Clear & $280 / 245$ & Šmelcer L. & $\begin{array}{l}\text { Celestron SCT } 280 / 1765+ \\
\text { MII G2-4000 }\end{array}$ \\
\hline 57902.52478 & 0.0001 & II & CCD + Clear & $226 / 177$ & Bílek F. & $\begin{array}{l}\text { NWT } 200 / 860+\text { CCD Atik } \\
314 \mathrm{~L}+\end{array}$ \\
\hline 57923.50886 & 0.0001 & II & CCD + Clear & $180 / 142$ & Bílek F. & $\begin{array}{l}\text { NWT 200/860 + CCD Atik } \\
314 \mathrm{~L}+\end{array}$ \\
\hline 57924.42928 & 0 & I & CCD + Clear & $185 / 72$ & Bílek F. & $\begin{array}{l}\text { NWT 200/860 + CCD Atik } \\
314 \mathrm{~L}+\end{array}$ \\
\hline 57929.39937 & 0 & II & $\mathrm{CCD}+\mathrm{R}$ & $158 / 29$ & Šmelcer L. & $\begin{array}{l}\text { Celestron SCT } 355 / 2460+ \\
\text { MII G2-1600 }\end{array}$ \\
\hline 57931.42397 & 0.0001 & I & CCD + Clear & $121 / 64$ & Bílek F. & $\begin{array}{l}\text { NWT 200/860 + CCD Atik } \\
314 \mathrm{~L}+\end{array}$ \\
\hline 57938.41860 & 0.0001 & I & $\mathrm{CCD}+\mathrm{R}$ & $175 / 79$ & Šmelcer L. & $\begin{array}{l}\text { Celestron SCT } 355 / 2460+ \\
\text { MII G2-1600 }\end{array}$ \\
\hline 57939.52302 & 0.0001 & I & CCD + Clear & $198 / 160$ & Bílek F. & $\begin{array}{l}\text { NWT 200/860 + CCD Atik } \\
\text { 314L+ }\end{array}$ \\
\hline 57941.36489 & 0.0001 & I & $\mathrm{CCD}+\mathrm{V}$ & $148 / 24$ & Šmelcer L. & $\begin{array}{l}\text { Celestron SCT } 355 / 2460+ \\
\text { MII G2-1600 }\end{array}$ \\
\hline 57948.35847 & 0.0001 & I & CCD + Clear & $224 / 15$ & Bílek F. & $\begin{array}{l}\text { NWT 200/860 }+ \text { CCD Atik } \\
314 \mathrm{~L}+\end{array}$ \\
\hline 57948.54193 & 0.0001 & II & CCD + Clear & $224 / 187$ & Bílek F. & $\begin{array}{l}\text { NWT 200/860 + CCD Atik } \\
314 \mathrm{~L}+\end{array}$ \\
\hline 57954.43284 & 0.0001 & I & $\mathrm{CCD}+\mathrm{V}$ & $216 / 84$ & Šmelcer L. & $\begin{array}{l}\text { Celestron SCT } 355 / 2460 \\
\text { MII G2-1600 }\end{array}$ \\
\hline 57956.45778 & 0.0001 & I & $\mathrm{CCD}+\mathrm{V}$ & $169 / 63$ & Šmelcer L. & $\begin{array}{l}\text { Celestron SCT } 355 / 2460 \\
\text { MII G2-1600 }\end{array}$ \\
\hline 57974.49619 & 0.0001 & I & $\mathrm{CCD}+\mathrm{V}$ & $229 / 176$ & Šmelcer L. & $\begin{array}{l}\text { Celestron SCT } 355 / 2460 \\
\text { MII G2-1600 }\end{array}$ \\
\hline 57980.38745 & 0.0001 & I & $\mathrm{CCD}+\mathrm{V}$ & $276 / 87$ & Šmelcer L. & $\begin{array}{l}\text { Celestron SCT } 355 / 2460 \\
\text { MII G2-1600 }\end{array}$ \\
\hline 57981.49129 & 0.0001 & I & $\mathrm{CCD}+\mathrm{R}$ & $314 / 229$ & Šmelcer L. & $\begin{array}{l}\text { Celestron SCT } 355 / 2460 \\
\text { MII G2-1600 }\end{array}$ \\
\hline 57995.48055 & 0.0001 & I & $\mathrm{CCD}+\mathrm{V}$ & $320 / 166$ & Šmelcer L. & $\begin{array}{l}\text { Celestron SCT } 355 / 2460 \\
\text { MII G2-1600 }\end{array}$ \\
\hline 58027.32442 & 0.0001 & II & $\mathrm{CCD}+\mathrm{V}$ & $367 / 89$ & Šmelcer L. & $\begin{array}{l}\text { Celestron SCT } 355 / 2460 \\
\text { MII G2-1600 }\end{array}$ \\
\hline 58027.50750 & 0.0003 & $\mathrm{I}$ & $\mathrm{CCD}+\mathrm{V}$ & $367 / 317$ & Šmelcer L. & $\begin{array}{l}\text { Celestron SCT } 355 / 2460 \\
\text { MII G2-1600 }\end{array}$ \\
\hline 58040.39366 & 0.0001 & $\mathrm{I}$ & $\mathrm{CCD}+\mathrm{V}$ & $239 / 156$ & Šmelcer L. & $\begin{array}{l}\text { Celestron SCT } 355 / 2460 \\
\text { MII G2-1600 }\end{array}$ \\
\hline 58042.23419 & 0.0001 & I & $\mathrm{CCD}+\mathrm{V}$ & $274 / 24$ & Šmelcer L. & $\begin{array}{l}\text { Celestron SCT } 355 / 2460 \\
\text { MII G2-1600 }\end{array}$ \\
\hline 58042.41875 & 0.0001 & II & $\mathrm{CCD}+\mathrm{V}$ & $274 / 245$ & Šmelcer L. & $\begin{array}{l}\text { Celestron SCT } 355 / 2460 \\
\text { MII G2-1600 }\end{array}$ \\
\hline 58063.40168 & 0.0003 & II & $\mathrm{CCD}+\mathrm{V}$ & $97 / 33$ & Šmelcer L. & $\begin{array}{l}\text { Celestron SCT } 355 / 2460 \\
\text { MII G2-1600 }\end{array}$ \\
\hline
\end{tabular}




\begin{tabular}{|c|c|c|c|c|c|c|}
\hline $\begin{array}{l}\text { Star } \\
\text { HJD-2400000 }\end{array}$ & Error & $\mathrm{I} / \mathrm{II}$ & Method & Points & Observer & Technique \\
\hline 58149.36338 & 0.0001 & I & $\mathrm{CCD}+\mathrm{V}$ & $542 / 214$ & Šmelcer L. & $\begin{array}{l}\text { Celestron SCT } 355 / 2460 \quad+ \\
\text { MII G2-1600 }\end{array}$ \\
\hline 58149.54777 & 0.0001 & II & $\mathrm{CCD}+\mathrm{V}$ & $542 / 444$ & Šmelcer L. & $\begin{array}{l}\text { Celestron SCT } 355 / 2460+ \\
\text { MII G2-1600 }\end{array}$ \\
\hline 58178.44566 & 0.0001 & I & $\mathrm{CCD}+\mathrm{V}$ & $399 / 151$ & Šmelcer L. & $\begin{array}{l}\text { Celestron SCT } 355 / 2460+ \\
\text { MII G2-1600 }\end{array}$ \\
\hline 58178.62987 & 0.0002 & II & $\mathrm{CCD}+\mathrm{V}$ & $399 / 379$ & Šmelcer L. & $\begin{array}{l}\text { Celestron SCT } 355 / 2460+ \\
\text { MII G2-1600 }\end{array}$ \\
\hline 58179.36560 & 0.0002 & II & $\mathrm{CCD}+\mathrm{V}$ & $182 / 161$ & Šmelcer L. & $\begin{array}{l}\text { Celestron SCT } 355 / 2460+ \\
\text { MII G2-1600 }\end{array}$ \\
\hline 58182.31121 & 0.0001 & II & CCD + Clear & $173 / 67$ & Bílek F. & $\begin{array}{l}\text { NWT 200/860 + CCD Atik } \\
314 \mathrm{~L}+\end{array}$ \\
\hline 58182.31123 & 0.0001 & II & $\mathrm{CCD}+\mathrm{V}$ & $435 / 100$ & Šmelcer L. & $\begin{array}{l}\text { Celestron SCT } 355 / 2460+ \\
\text { MII G2-1600 }\end{array}$ \\
\hline 58182.49524 & 0.0001 & I & $\mathrm{CCD}+\mathrm{V}$ & $435 / 338$ & Šmelcer L. & $\begin{array}{l}\text { Celestron SCT } 355 / 2460+ \\
\text { MII G2-1600 }\end{array}$ \\
\hline 58186.36069 & 0.0001 & II & CCD + Clear & $210 / 111$ & Bílek F. & $\begin{array}{l}\text { NWT } 200 / 860+\text { CCD Atik } \\
314 \mathrm{~L}+\end{array}$ \\
\hline 58198.32562 & 0.0001 & I & CCD +Clear & $135 / 64$ & Bílek F. & $\begin{array}{l}\text { NWT 200/860 + CCD Atik } \\
314 \mathrm{~L}+\end{array}$ \\
\hline 58199.42995 & 0.0001 & I & CCD + Clear & $375 / 159$ & Bílek F. & $\begin{array}{l}\text { NWT 200/860 + CCD Atik } \\
314 \mathrm{~L}+\end{array}$ \\
\hline 58199.61385 & 0.0001 & II & CCD + Clear & $375 / 331$ & Bílek F. & $\begin{array}{l}\text { NWT 200/860 + CCD Atik } \\
314 \mathrm{~L}+\end{array}$ \\
\hline 58202.37509 & 0.0001 & I & CCD + Clear & $340 / 106$ & Bílek F. & $\begin{array}{l}\text { NWT 200/860 + CCD Atik } \\
314 \mathrm{~L}+\end{array}$ \\
\hline 58202.55900 & 0.0001 & II & CCD + Clear & $340 / 277$ & Bílek F. & $\begin{array}{l}\text { NWT } 200 / 860+\text { CCD Atik } \\
314 \mathrm{~L}+\end{array}$ \\
\hline
\end{tabular}

NSVS 547680 Cam 57852.35496

NSVS 609693 Cam 57822.44178

NSVS 755884 Cam 57780.64166

OQ Cam 58117.47291
0.0009 II $\quad$ CCD + Clear $\quad$ 168/94 Bragagnolo U.

Sky-Watcher REF 100/900 + QHY9

Sky-Watcher REF 100/900 + QHY9

0.0003 I CCD + Clear 309/252 Bragagnolo U.

0.0003 I CCD + Clear 241/149 Bragagnolo U.

Sky-Watcher REF 100/900 + QHY9

REF $120 / 600+$ Orion 3G $\mathrm{CCD}+0.5 \mathrm{x}$ reducer 


\begin{abstract}
Star
HJD-2400000

QU Cam

57775.41423

57884.43040

SV Cam

58047.40926

UU Cam
\end{abstract}

57844.34436

V0335 Cam

58065.33487

V0337 Cam

57728.26290

58042.32438

V0345 Cam

57728.28624

V0352 Cam

58073.41337

V0353 Cam

58073.29289

57803.36692

58093.57480
58080.41368

V0355 Cam

$\begin{array}{lllll}0.0001 & \text { I } & \text { CCD }+ \text { Clear } & 230 / 112 & \text { Walter F. } \\ 0.0001 \text { II } & \text { CCD }+ \text { Clear } & 168 / 79 & \text { Tylšar M. }\end{array}$

0.0009 II CCD + Clear 110/57 Vrašták M.

0.0002 I CCD + Clear 273/143 Bragagnolo U.

Error I/II Method

Points Observer

Technique

0.0005 I $\quad$ CCD + Clear $\quad 67 / 26$

Tylšar M., Ma- NWT250/1000,G2-8300 šek M.

0.0002 I CCD + Clear $\quad 78 / 43 \quad$ Urbaník M.

0.0003 I DSLR

210/54 Školník V.

0.0007 I CCD + Clear 274/186 Urbaník M.

Sky-Watcher REF 80/520 + MII G2-8300

Sky-Watcher REF $80 / 520+$ MII G2-8300

Tamron LNS 44/300 + Canon 600D

Sky-Watcher REF 100/900 + QHY9

DATEL, NWT 200/800 + MII G2 8300

DATEL, NWT 200/800 + MII G2 8300

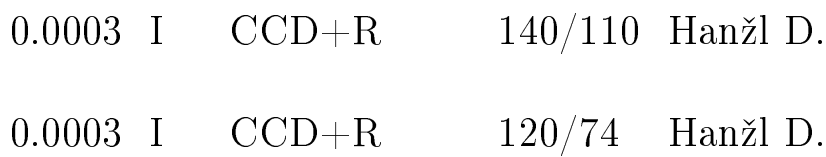

134/29 Hanžl D.

DATEL, NWT 200/800 + MII G2 8300

0.0004 II CCD+Clear 368/177 Bragagnolo U. Sky-Watcher REF 100/900 QHY9

0.0005 I CCD + Clear 266/129 Bragagnolo U. Sky-Watcher REF 100/900 + QHY9

V0356 Cam

58073.33727
$0.0003 \mathrm{I} \quad \mathrm{CCD}+\mathrm{R} \quad 141 / 65 \quad$ Hanžl D.
DATEL, NWT 200/800 + MII

G2 8300 


\begin{tabular}{|c|c|c|c|c|c|}
\hline Star & & & & & \\
\hline HJD-2400000 & Error & I/II Method & Points & Observer & Technique \\
\hline 58080.38326 & 0.0003 & I $\quad \mathrm{CCD}+\mathrm{R}$ & $119 / 54$ & Hanžl D. & $\begin{array}{l}\text { DATEL, NWT } 200 / 800+\text { MII } \\
\text { G2 } 8300\end{array}$ \\
\hline
\end{tabular}

V0368 Cam 58026.40967

V0382 Cam 57755.53674

V0383 Cam 57822.34391

V0386 Cam 57778.63622 57823.34477 V0389 Cam 57852.36228 V0394 Cam 58113.32078 V0396 Cam 58113.31932

V0406 Cam 57801.37298 58027.41069 58028.28272 58028.43002 58084.35601
0.0003 I $\quad$ CCD + R 139/116 Hanžl D.

0.0002 I CCD + Clear 209/116 Bragagnolo U.
DATEL, NWT 200/800 + MII G2 8300

Sky-Watcher REF 100/900 QHY9

Sky-Watcher REF 100/900 + QHY9

$\begin{array}{lll}0.0003 \text { I CCD }+ \text { Clear } 277 / 195 \text { Bragagnolo U. } \begin{array}{l}\text { Sky-Watcher REF 100/900 + } \\ \text { QHY9 }\end{array} & \end{array}$ 0.0003 I CCD+Clear 403/111 Bragagnolo U. Sky-Watcher REF 100/900 + QHY9

0.0003 II CCD +Clear 174/124 Bragagnolo U. Sky-Watcher REF 100/900 + QHY9

0.0002 II $\quad \mathrm{CCD}+\mathrm{R} \quad 120 / 71 \quad$ Hanžl D.

DATEL, NWT 200/800 + MII G2 8300

DATEL, NWT 200/800 + MII G2 8300
Sky-Watcher REF 100/900 + QHY9
DATEL, NWT 200/800 + MII G2 8300
DATEL, NWT 200/800 + MII G2 8300
DATEL, NWT 200/800 + MII G2 8300
0.0004 I $\quad C C D+$ Clear $\quad 174 / 123$ Bragagnolo U.
$0.0003 \mathrm{I} \quad \mathrm{CCD}+\mathrm{R} \quad 160 / 89 \quad$ Hanžl D.
186/22 Hanžl D.
0.0003 II $\mathrm{CCD}+\mathrm{R} \quad$ 186/123 Hanžl D.

DATEL, NWT 200/800 + MII 


\begin{tabular}{lcllll}
\hline $\begin{array}{l}\text { Star } \\
\text { HJD-2400000 }\end{array}$ & Error & I/II Method & Points & Observer & Technique \\
\hline $\begin{array}{l}\text { V0452 Cam } \\
57814.31579\end{array}$ & 0.0005 I & CCD +Clear & $370 / 71$ & Bragagnolo U. & $\begin{array}{l}\text { Sky-Watcher REF } 100 / 900+ \\
\text { QHY9 }\end{array}$ \\
57814.51858 & 0.0008 II & CCD +Clear & $370 / 296$ & Bragagnolo U. & $\begin{array}{l}\text { Sky-Watcher REF } 100 / 900+ \\
\text { QHY9 }\end{array}$
\end{tabular}

V0456 Cam 57746.58706

57852.37912

V0459 Cam 57841.53486

V0461 Cam 57722.36974 57722.53181 V0466 Cam 58089.38939

V0470 Cam 58046.39296 58046.44110 58046.48857 V0473 Cam 57744.54507 57744.69755 57821.24458 V0474 Cam 57760.66859 57760.66888
0.0005 I $\quad$ CCD + Clear

339/107 Bragagnolo U. 0.0006 I CCD + Clear $80 / 16$ Audejean M.

0.0002 I CCD + Clear 466/377 Bragagnolo U.

Sky-Watcher REF 100/900 QHY9

$\begin{array}{lllll}0.0003 & \text { I } & \text { CCD }+\mathrm{R} & 311 / 87 & \text { Hanžl D. } \\ 0.0008 & \text { II } & \mathrm{CCD}+\mathrm{R} & 311 / 196 & \text { Hanžl D. }\end{array}$

DATEL, NWT 200/800 + MII G2 8300

DATEL, NWT 200/800 + MII G2 8300

DATEL, NWT 200/800 + MII G2 8300

0.0003 I $\quad \mathrm{CCD}+\mathrm{R} \quad 133 / 102$ Hanžl D.

DATEL, NWT 200/800 + MII G2 8300

DATEL, NWT 200/800 + MII G2 8300

DATEL, NWT 200/800 + MII G2 8300

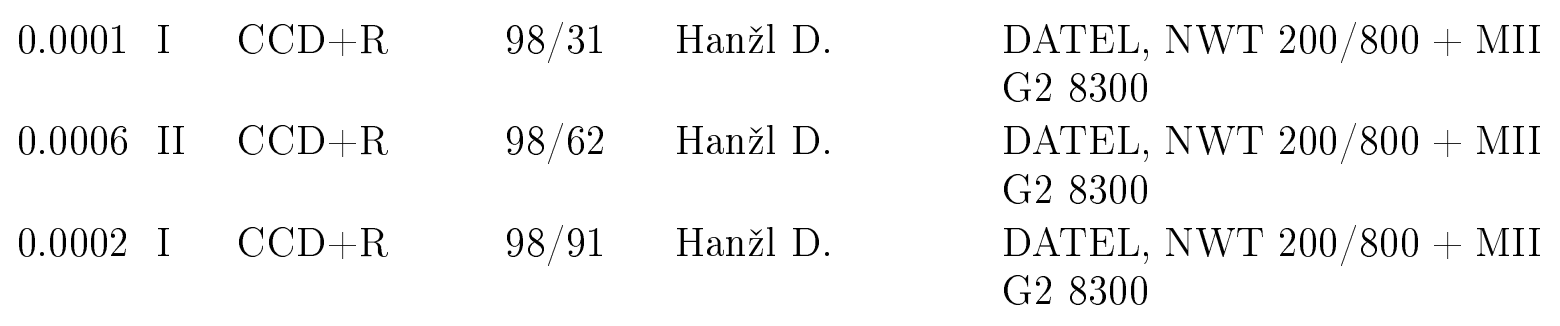

0.0003 II CCD + Clear 286/51 Bragagnolo U. Sky-Watcher REF 100/900 + QHY9

0.0002 I CCD + Clear 286/253 Bragagnolo U.

Sky-Watcher REF 100/900 + QHY9

0.0002 II CCD+Clear 154/22 Urbaník M.

Sky-Watcher NWT 150/750 + MII G2-8300

Kometensucher Zeiss REF $200 / 1370+$ SBIG ST7

CZ Mirotar SCT 180/1000, Canon 350d 


\begin{tabular}{llllllll}
\hline $\begin{array}{l}\text { Star } \\
\text { HJD-2400000 }\end{array}$ & Error & I/II Method & Points & Observer & Technique & \\
\hline 57764.44222 & 0.0002 & I & DSLR & $356 / 101$ & Walter F. & $\begin{array}{l}\text { CZ Mirotar SCT } \\
\text { Canon 350d }\end{array}$ \\
57764.44344 & 0.0002 & II & CCD + Clear & $218 / 42$ & Walter F. & $\begin{array}{l}\text { Kometensucher Zeiss } \\
\text { 200/1370 + SBIG ST7 }\end{array}$
\end{tabular}

V0478 Cam 57821.31409

V0479 Cam 58117.47801

V0488 Cam 57775.50975

V0490 Cam 58026.65365

V0503 Cam 57780.61062

V0505 Cam 57773.32757

V0511 Cam 57843.35578

V466 Cam 57405.35655

WW Cam 58095.39859 58113.59278
0.0005 I CCD + Clear 153/125 Urbaník M.

$0.0003 \mathrm{I} \quad \mathrm{CCD}+\mathrm{R} \quad 116 / 98$ Hanžl D.

0.0007 II $\quad$ CCD + Clear 193/77 Bragagnolo U.

0.0003 I $\quad$ CCD + Clear $\quad 80 / 70 \quad$ Walter F.

0.0002 I $\quad$ CCD + Clear 242/105 Bragagnolo U.

Sky-Watcher REF 100/900 + QHY9

0.0008 I $\quad$ CCD + Clear $102 / 33$ Walter F.

0.0004 I CCD + Clear 587/295 Bragagnolo U.

Sky-Watcher REF 100/900 + QHY9

MARK SCT 406/4060, SBIG ST10XME

DATEL, NWT 200/800 + MII G2 8300

Sky-Watcher REF 100/900 + QHY9

MARK SCT 406/4060, SBIG ST10XME

AB Cas 57773.43748
0.0001 I CCD + Clear $\quad 175 / 78$ Bragagnolo U. Sky-Watcher REF 100/900 + QHY9 


\begin{tabular}{|c|c|c|c|c|c|}
\hline Star & & & & & \\
\hline HJD-2400000 & Error & I/II Method & Points & Observer & Technique \\
\hline 57780.27185 & 0.0001 & I $\quad$ CCD + Clear & $163 / 62$ & Bragagnolo U. & $\begin{array}{l}\text { Sky-Watcher REF } 100 / 900+ \\
\text { QHY9 }\end{array}$ \\
\hline
\end{tabular}

BS Cas

57740.29144

57811.42588

57811.42604

57811.64733

58028.35513

CW Cas

58054.53159

CzeV590 Cas 57627.43655

57628.42988

57631.40662

57632.39907

57632.56292

57633.39160

57634.38367

57634.54884

57639.34562

57639.51064

57640.33891

57640.50324

57642.32352

57642.48787
0.0001 II $\mathrm{CCD}+\mathrm{R}$

$0.0003 \mathrm{I} \quad \mathrm{CCD}+\mathrm{V} \quad 119 / 56 \quad$ Walter F.

0.0003 I $\quad$ CCD + Clear $\quad 176 / 59$ Walter F.

0.0005 II CCD + Clear 176/133 Walter F.

0.0001 II DSLR

0.0001 I CCD + Clear 156/53 Bragagnolo U.
NWT $250 / 1000+$ ATIK $314 \mathrm{~L}++\mathrm{MPCC}$

Set sekce, NWT 150/750 + G2-1600

Set sekce, NWT 150/750 + G2-1600

Set sekce, NWT 150/750 G2-1600

NWT 150/600 + Canon 20D + MPCC mk.III

Sky-Watcher REF 100/900 + QHY9

NWT 200/860 + CCD Atik $314 \mathrm{~L}+$

NWT 200/860 + CCD Atik $314 \mathrm{~L}+$

NWT 200/860 + CCD Atik $314 \mathrm{~L}+$

NWT 200/860 + CCD Atik $314 \mathrm{~L}+$

NWT 200/860 + CCD Atik $314 \mathrm{~L}+$

NWT 200/860 + CCD Atik $314 \mathrm{~L}+$

NWT 200/860 + CCD Atik $314 \mathrm{~L}+$

NWT 200/860 + CCD Atik $314 \mathrm{~L}+$

NWT 200/860 + CCD Atik $314 \mathrm{~L}+$

NWT 200/860 + CCD Atik $314 \mathrm{~L}+$

NWT 200/860 + CCD Atik $314 \mathrm{~L}+$

NWT 200/860 + CCD Atik $314 \mathrm{~L}+$

NWT 200/860 + CCD Atik $314 \mathrm{~L}+$

NWT 200/860 + CCD Atik $314 \mathrm{~L}+$ 


\begin{tabular}{|c|c|c|c|c|c|c|}
\hline $\begin{array}{l}\text { Star } \\
\text { HJD-2400000 }\end{array}$ & Error & & Method & Points & Observer & Technique \\
\hline 57644.47262 & 0.0006 & II & CCD + Clear & $337 / 193$ & Bílek F. & $\begin{array}{l}\text { NWT } 200 / 860+\text { CCD Atik } \\
314 \mathrm{~L}+\end{array}$ \\
\hline 57646.45883 & 0.0006 & II & $\mathrm{CCD}+$ Clear & $337 / 177$ & Bílek F. & $\begin{array}{l}\text { NWT } 200 / 860+\text { CCD Atik } \\
314 \mathrm{~L}+\end{array}$ \\
\hline 57656.38245 & 0.0006 & II & $\mathrm{CCD}+$ Clear & $313 / 127$ & Bílek F. & $\begin{array}{l}\text { NWT } 200 / 860+\text { CCD Atik } \\
314 \mathrm{~L}+\end{array}$ \\
\hline 57658.53244 & 0.0005 & I & $\mathrm{CCD}+$ Clear & $343 / 240$ & Bílek F. & $\begin{array}{l}\text { NWT 200/860 }+ \text { CCD Atik } \\
314 \mathrm{~L}+\end{array}$ \\
\hline 57661.34464 & 0.0006 & II & CCD + Clear & $368 / 97$ & Bílek F. & $\begin{array}{l}\text { NWT } 200 / 860+\text { CCD Atik } \\
314 \mathrm{~L}+\end{array}$ \\
\hline 57661.51069 & 0.0005 & I & $\mathrm{CCD}+$ Clear & $368 / 249$ & Bílek F. & $\begin{array}{l}\text { NWT 200/860 + CCD Atik } \\
\text { 314L }+\end{array}$ \\
\hline 57714.27417 & 0.0008 & II & CCD + Clear & $192 / 73$ & Bílek F. & $\begin{array}{l}\text { NWT } 200 / 860+\text { CCD Atik } \\
314 \mathrm{~L}+\end{array}$ \\
\hline 57715.59842 & 0.0006 & I & $\mathrm{CCD}+\mathrm{R}$ & $274 / 239$ & Vrašták M & $\begin{array}{l}\text { NWT } 240 / 1200+\text { MII G2-1600, } \\
80 / 400+\text { G1-0300 }\end{array}$ \\
\hline 57722.38004 & 0.0004 & I & $\mathrm{CCD}+\mathrm{R}$ & $376 / 217$ & Lehky M. & $\begin{array}{l}\text { BlueEye600, RiLA RCT } \\
600 / 3000+\text { MII G4-9000CE } \\
+\end{array}$ \\
\hline 57722.38018 & 0.0005 & I & $\mathrm{CCD}+$ Clear & $463 / 182$ & Bílek F. & $\begin{array}{l}\text { NWT } 200 / 860+\text { CCD Atik } \\
314 \mathrm{~L}+\end{array}$ \\
\hline 57722.54514 & 0.0005 & II & $\mathrm{CCD}+$ Clear & $463 / 331$ & Bílek F. & $\begin{array}{l}\text { NWT } 200 / 860+\text { CCD Atik } \\
314 \mathrm{~L}+\end{array}$ \\
\hline 57725.35737 & 0.0005 & I & $\mathrm{CCD}+$ Clear & $517 / 170$ & Bílek F. & $\begin{array}{l}\text { NWT 200/860 + CCD Atik } \\
\text { 314L+ }\end{array}$ \\
\hline 57725.52184 & 0.0006 & II & $\mathrm{CCD}+$ Clear & $517 / 324$ & Bílek F. & $\begin{array}{l}\text { NWT } 200 / 860+\text { CCD Atik } \\
314 \mathrm{~L}+\end{array}$ \\
\hline 57725.68786 & 0.0006 & I & $\mathrm{CCD}+$ Clear & $517 / 477$ & Bílek F. & $\begin{array}{l}\text { NWT } 200 / 860+\text { CCD Atik } \\
314 \mathrm{~L}+\end{array}$ \\
\hline 57726.35012 & 0.0006 & I & $\mathrm{CCD}+$ Clear & $402 / 156$ & Bílek F. & $\begin{array}{l}\text { NWT } 200 / 860+\text { CCD Atik } \\
314 \mathrm{~L}+\end{array}$ \\
\hline 57726.51446 & 0.0009 & II & CCD + Clear & $402 / 304$ & Bílek F. & $\begin{array}{l}\text { NWT } 200 / 860+\text { CCD Atik } \\
314 \mathrm{~L}+\end{array}$ \\
\hline 57733.29637 & 0.0005 & I & CCD + Clear & $456 / 109$ & Bílek F. & $\begin{array}{l}\text { NWT } 200 / 860+\text { CCD Atik } \\
314 \mathrm{~L}+\end{array}$ \\
\hline 57733.46173 & 0.0006 & II & CCD + Clear & $456 / 256$ & Bílek F. & $\begin{array}{l}\text { NWT 200/860 + CCD Atik } \\
314 \mathrm{~L}+\end{array}$ \\
\hline 57733.62772 & 0.0007 & I & CCD + Clear & $456 / 400$ & Bílek F. & $\begin{array}{l}\text { NWT } 200 / 860+\text { CCD Atik } \\
314 \mathrm{~L}+\end{array}$ \\
\hline 57739.25277 & 0.0005 & I & CCD + Clear & $370 / 73$ & Bílek F. & $\begin{array}{l}\text { NWT } 200 / 860+\text { CCD Atik } \\
314 \mathrm{~L}+\end{array}$ \\
\hline 57753.47699 & 0.0005 & I & CCD + Clear & $505 / 268$ & Bílek F. & $\begin{array}{l}\text { NWT } 200 / 860+\text { CCD Atik } \\
314 \mathrm{~L}+\end{array}$ \\
\hline 57753.64125 & 0.0005 & II & CCD + Clear & $505 / 419$ & Bílek F. & $\begin{array}{l}\text { NWT 200/860 + CCD Atik } \\
314 \mathrm{~L}+\end{array}$ \\
\hline 57754.30344 & 0.0005 & II & CCD + Clear & $470 / 115$ & Bílek F. & $\begin{array}{l}\text { NWT } 200 / 860+\text { CCD Atik } \\
314 \mathrm{~L}+\end{array}$ \\
\hline
\end{tabular}




\begin{tabular}{|c|c|c|c|c|c|c|}
\hline \multicolumn{7}{|l|}{ Star } \\
\hline HJD-2400000 & Error & I/II & Method & Points & Observer & Technique \\
\hline 57754.46812 & 0.0006 & I & $\mathrm{CCD}+$ Clear & $470 / 262$ & Bílek F. & $\begin{array}{l}\text { NWT 200/860 + CCD Atik } \\
314 \mathrm{~L}+\end{array}$ \\
\hline 57754.63335 & 0.0005 & II & $\mathrm{CCD}+$ Clear & $470 / 415$ & Bílek F. & $\begin{array}{l}\text { NWT } 200 / 860+\text { CCD Atik } \\
314 \mathrm{~L}+\end{array}$ \\
\hline 57755.29610 & 0.0005 & II & CCD + Clear & $450 / 97$ & Bílek F. & $\begin{array}{l}\text { NWT 200/860 + CCD Atik } \\
314 \mathrm{~L}+\end{array}$ \\
\hline 57755.46098 & 0.0005 & I & $\mathrm{CCD}+$ Clear & $450 / 248$ & Bílek F. & $\begin{array}{l}\text { NWT } 200 / 860+\text { CCD Atik } \\
314 \mathrm{~L}+\end{array}$ \\
\hline 57755.62721 & 0.0005 & II & $\mathrm{CCD}+$ Clear & $450 / 399$ & Bílek F. & $\begin{array}{l}\text { NWT } 200 / 860+\text { CCD Atik } \\
314 \mathrm{~L}+\end{array}$ \\
\hline 57772.33367 & 0.0005 & I & CCD + Clear & $312 / 139$ & Bílek F. & $\begin{array}{l}\text { NWT } 200 / 860+\text { CCD Atik } \\
314 \mathrm{~L}+\end{array}$ \\
\hline 57773.32586 & 0.0008 & I & $\mathrm{CCD}+\mathrm{V}$ & $306 / 103$ & Lehky M. & $\begin{array}{l}\text { BlueEye600, RiLA RCT } \\
600 / 3000+\text { MII G4-9000EC } \\
+\end{array}$ \\
\hline 57773.32600 & 0.0005 & I & CCD + Clear & $462 / 131$ & Bílek F. & $\begin{array}{l}\text { NWT } 200 / 860+\text { CCD Atik } \\
314 \mathrm{~L}+\end{array}$ \\
\hline 57773.49092 & 0.0007 & II & CCD + Clear & $462 / 298$ & Bílek F. & $\begin{array}{l}\text { NWT } 200 / 860+\text { CCD Atik } \\
314 \mathrm{~L}+\end{array}$ \\
\hline 57773.49388 & 0.0009 & II & $\mathrm{CCD}+\mathrm{V}$ & $306 / 244$ & Lehky M. & $\begin{array}{l}\text { BlueEye600, RiLA RCT } \\
600 / 3000+\text { MII G4-9000EC } \\
+\end{array}$ \\
\hline 57774.31899 & 0.0006 & I & $\mathrm{CCD}+$ Clear & $454 / 124$ & Bílek F. & $\begin{array}{l}\text { NWT 200/860 }+ \text { CCD Atik } \\
314 \mathrm{~L}+\end{array}$ \\
\hline 57774.48314 & 0.0005 & II & $\mathrm{CCD}+$ Clear & $454 / 294$ & Bílek F. & $\begin{array}{l}\text { NWT } 200 / 860+\text { CCD Atik } \\
314 \mathrm{~L}+\end{array}$ \\
\hline 57775.31023 & 0.0004 & I & CCD + Clear & $400 / 120$ & Bílek F. & $\begin{array}{l}\text { NWT } 200 / 860+\text { CCD Atik } \\
314 \mathrm{~L}+\end{array}$ \\
\hline 57775.31136 & 0.0007 & I & $\mathrm{CCD}+$ Clear & $551 / 131$ & Šmelcer L. & $\begin{array}{l}\text { Celestron SCT } 280 / 1765+ \\
\text { MII G2- } 4000\end{array}$ \\
\hline 57775.47664 & 0.0012 & II & $\mathrm{CCD}+$ Clear & $551 / 294$ & Šmelcer L. & $\begin{array}{l}\text { Celestron SCT } 280 / 1765+ \\
\text { MII G2-4000 }\end{array}$ \\
\hline 57775.64020 & 0.0007 & I & CCD + Clear & $551 / 473$ & Šmelcer L. & $\begin{array}{l}\text { Celestron SCT 280/1765 + } \\
\text { MII G2-4000 }\end{array}$ \\
\hline 57776.46802 & 0.0006 & II & $\mathrm{CCD}+$ Clear & $422 / 249$ & Bílek F. & $\begin{array}{l}\text { NWT } 200 / 860+\text { CCD Atik } \\
314 \mathrm{~L}+\end{array}$ \\
\hline 57798.30222 & 0.0006 & II & $\mathrm{CCD}+$ Clear & $373 / 72$ & Bílek F. & $\begin{array}{l}\text { NWT } 200 / 860+\text { CCD Atik } \\
314 \mathrm{~L}+\end{array}$ \\
\hline 57799.29472 & 0.0006 & II & CCD + Clear & $400 / 68$ & Bílek F. & $\begin{array}{l}\text { NWT } 200 / 860+\text { CCD Atik } \\
314 \mathrm{~L}+\end{array}$ \\
\hline 57800.28718 & 0.0006 & II & CCD + Clear & $398 / 63$ & Bílek F. & $\begin{array}{l}\text { NWT } 200 / 860+\text { CCD Atik } \\
314 \mathrm{~L}+\end{array}$ \\
\hline 57800.45222 & 0.0005 & I & CCD + Clear & $398 / 211$ & Bílek F. & $\begin{array}{l}\text { NWT } 200 / 860+\text { CCD Atik } \\
314 \mathrm{~L}+\end{array}$ \\
\hline 57801.27935 & 0.0004 & II & CCD + Clear & $228 / 54$ & Bílek F. & $\begin{array}{l}\text { NWT } 200 / 860+\text { CCD Atik } \\
314 \mathrm{~L}+\end{array}$ \\
\hline 57812.36204 & 0.0004 & I & $\mathrm{CCD}+$ Clear & $244 / 118$ & Bílek F. & $\begin{array}{l}\text { NWT } 200 / 860+\text { CCD Atik } \\
314 \mathrm{~L}+\end{array}$ \\
\hline
\end{tabular}




\begin{tabular}{llllll}
\hline $\begin{array}{l}\text { Star } \\
\text { HJD-2400000 }\end{array}$ & Error & I/II Method & Points & Observer \\
\hline 57829.39942 & 0.0006 & II & CCD + Clear & $311 / 139$ & Bílek F. \\
57838.33146 & 0.0006 & II & CCD + Clear & $120 / 62$ & Bílek F. \\
57987.53254 & 0.0005 & II & CCD + Clear & $75 / 57$ & Šmelcer L. \\
57989.51480 & 0.0004 & II & CCD + Clear & $154 / 47$ & Šmelcer L. \\
57991.49953 & 0.0005 & II & CCD + Clear & $311 / 174$ & Šmelcer L. \\
57995.46964 & 0.0005 & II & CCD + Clear & $325 / 166$ & Šmelcer L. \\
58027.39154 & 0.0006 & I & CCD + Clear & $298 / 133$ & Šmelcer L. \\
58042.27794 & 0.0004 & I & CCD + Clear & $268 / 61$ & Šmelcer L. \\
58043.27133 & 0.0007 & I & CCD + Clear & $379 / 76$ & Šmelcer L. \\
58043.43690 & 0.0004 & II & CCD + Clear & $379 / 277$ & Šmelcer L.
\end{tabular}

CzeV772 Cas 57627.40304

57628.41000

57631.44239

57632.45023

57633.46082

57634.47029

57639.52224

57640.53168

57642.35230

57642.55197

57644.37053

57644.57412

57645.58339
0.0006 I $\quad$ CCD + Clear 318/105 Bílek F

0.001 II CCD+Clear 312/111 Bílek F.

0.0008 I $\quad$ CCD + Clear 321/149 Bílek F.

0.0008 II CCD + Clear 335/161 Bílek F.

0.0007 I CCD+Clear 219/161 Bílek F.

0.0008 II CCD + Clear $\quad 320 / 180$ Bílek F .

0.0007 I CCD + Clear 334/230 Bílek F.

0.0007 II CCD + Clear 338/238 Bílek F.

0.0009 I CCD + Clear 342/76 Bílek F

0.0007 II CCD + Clear 342/258 Bílek F.

0.0008 I CCD+Clear 336/101 Bílek F.

0.0009 II CCD + Clear 336/283 Bílek F.

0.0006 I CCD+Clear 343/299 Bílek F.
Technique

NWT 200/860 + CCD Atik $314 \mathrm{~L}+$

NWT 200/860 + CCD Atik $314 \mathrm{~L}+$

Celestron SCT 280/1765 +

MII G2-4000

Celestron SCT 280/1765 +

MII G2-4000

Celestron SCT 280/1765

MII G2-4000

Celestron SCT 280/1765 +

MII G2-4000

Celestron SCT 280/1765

MII G2-4000

Celestron SCT 280/1765 +

MII G2-4000

Celestron SCT 280/1765 +

MII G2-4000

Celestron SCT 280/1765 +

MII G2-4000

NWT 200/860 + CCD Atik $314 \mathrm{~L}+$

NWT 200/860 + CCD Atik $314 \mathrm{~L}+$

NWT 200/860 + CCD Atik $314 \mathrm{~L}+$

NWT 200/860 + CCD Atik $314 \mathrm{~L}+$

NWT 200/860 + CCD Atik $314 \mathrm{~L}+$

NWT 200/860 + CCD Atik $314 \mathrm{~L}+$

NWT 200/860 + CCD Atik $314 \mathrm{~L}+$

NWT 200/860 + CCD Atik $314 \mathrm{~L}+$

NWT 200/860 + CCD Atik $314 \mathrm{~L}+$

NWT 200/860 + CCD Atik $314 \mathrm{~L}+$

NWT 200/860 + CCD Atik $314 \mathrm{~L}+$

NWT 200/860 + CCD Atik $314 \mathrm{~L}+$

NWT 200/860 + CCD Atik $314 \mathrm{~L}+$ 


\begin{tabular}{|c|c|c|c|c|c|c|}
\hline $\begin{array}{l}\text { Star } \\
\text { HJD-2400000 }\end{array}$ & Error & $\mathrm{I} / \mathrm{Il}$ & Method & Points & Observer & Technique \\
\hline 57656.49309 & 0.0007 & I & CCD + Clear & $299 / 230$ & Bílek F. & $\begin{array}{l}\text { NWT } 200 / 860+\text { CCD Atik } \\
314 \mathrm{~L}+\end{array}$ \\
\hline 57658.51327 & 0.0006 & I & $\mathrm{CCD}+$ Clear & $338 / 220$ & Bílek F. & $\begin{array}{l}\text { NWT } 200 / 860+\text { CCD Atik } \\
314 \mathrm{~L}+\end{array}$ \\
\hline 57661.34262 & 0.0008 & I & CCD + Clear & $370 / 94$ & Bílek F. & $\begin{array}{l}\text { NWT } 200 / 860+\text { CCD Atik } \\
314 \mathrm{~L}+\end{array}$ \\
\hline 57661.54352 & 0.0007 & II & $\mathrm{CCD}+$ Clear & $370 / 278$ & Bílek F. & $\begin{array}{l}\text { NWT } 200 / 860+\text { CCD Atik } \\
314 \mathrm{~L}+\end{array}$ \\
\hline 57714.27590 & 0.0007 & I & CCD + Clear & $193 / 75$ & Bílek F. & $\begin{array}{l}\text { NWT } 200 / 860+\text { CCD Atik } \\
314 \mathrm{~L}+\end{array}$ \\
\hline 57722.35811 & 0.0006 & I & CCD + Clear & $468 / 163$ & Bílek F. & $\begin{array}{l}\text { NWT } 200 / 860+\text { CCD Atik } \\
314 \mathrm{~L}+\end{array}$ \\
\hline 57722.36001 & 0.0007 & I & $\mathrm{CCD}+\mathrm{R}$ & $373 / 191$ & Lehky M. & $\begin{array}{l}\text { BlueEye600, RiLA RCT } \\
600 / 3000+\text { MII G4-9000CE } \\
+\end{array}$ \\
\hline 57722.55709 & 0.0009 & II & CCD + Clear & $468 / 346$ & Bílek F. & $\begin{array}{l}\text { NWT 200/860 }+ \text { CCD Atik } \\
314 \mathrm{~L}+\end{array}$ \\
\hline 57725.38344 & 0.0008 & II & CCD + Clear & $506 / 189$ & Bílek F. & $\begin{array}{l}\text { NWT } 200 / 860+\text { CCD Atik } \\
314 \mathrm{~L}+\end{array}$ \\
\hline 57725.59044 & 0.0006 & I & $\mathrm{CCD}+$ Clear & $506 / 374$ & Bílek F. & $\begin{array}{l}\text { NWT 200/860 }+ \text { CCD Atik } \\
314 \mathrm{~L}+\end{array}$ \\
\hline 57726.39685 & 0.0008 & I & CCD + Clear & $404 / 203$ & Bílek F. & $\begin{array}{l}\text { NWT } 200 / 860+\text { CCD Atik } \\
314 \mathrm{~L}+\end{array}$ \\
\hline 57733.26485 & 0.0008 & I & CCD + Clear & $466 / 82$ & Bílek F. & $\begin{array}{l}\text { NWT } 200 / 860+\text { CCD Atik } \\
314 \mathrm{~L}+\end{array}$ \\
\hline 57733.46864 & 0.001 & II & CCD + Clear & $466 / 269$ & Bílek F. & $\begin{array}{l}\text { NWT } 200 / 860+\text { CCD Atik } \\
314 \mathrm{~L}+\end{array}$ \\
\hline 57739.32612 & 0.0008 & I & CCD + Clear & $368 / 141$ & Bílek F. & $\begin{array}{l}\text { NWT } 200 / 860+\text { CCD Atik } \\
314 \mathrm{~L}+\end{array}$ \\
\hline 57753.46817 & 0.0005 & I & CCD + Clear & $500 / 261$ & Bílek F. & $\begin{array}{l}\text { NWT } 200 / 860+\text { CCD Atik } \\
314 \mathrm{~L}+\end{array}$ \\
\hline 57753.66802 & 0.0008 & II & CCD + Clear & $500 / 442$ & Bílek F. & $\begin{array}{l}\text { NWT } 200 / 860+\text { CCD Atik } \\
314 \mathrm{~L}+\end{array}$ \\
\hline 57754.27805 & 0.0006 & I & CCD + Clear & $470 / 93$ & Bílek F. & $\begin{array}{l}\text { NWT } 200 / 860+\text { CCD Atik } \\
314 \mathrm{~L}+\end{array}$ \\
\hline 57754.47709 & 0.0007 & II & CCD + Clear & $470 / 271$ & Bílek F. & $\begin{array}{l}\text { NWT } 200 / 860+\text { CCD Atik } \\
314 \mathrm{~L}+\end{array}$ \\
\hline 57755.28590 & 0.0006 & II & CCD + Clear & $456 / 94$ & Bílek F. & $\begin{array}{l}\text { NWT } 200 / 860+\text { CCD Atik } \\
314 \mathrm{~L}+\end{array}$ \\
\hline 57755.48990 & 0.0008 & I & CCD + Clear & $456 / 282$ & Bílek F. & $\begin{array}{l}\text { NWT 200/860 }+ \text { CCD Atik } \\
314 \mathrm{~L}+\end{array}$ \\
\hline 57773.26169 & 0.0008 & I & $\mathrm{CCD}+\mathrm{V}$ & $318 / 51$ & Lehky M. & $\begin{array}{l}\text { BlueEye600, RiLA RCT } \\
600 / 3000+\text { MII G4-9000EC } \\
+\end{array}$ \\
\hline 57773.46778 & 0.0013 & II & CCD + Clear & $460 / 273$ & Bílek F. & $\begin{array}{l}\text { NWT } 200 / 860+\text { CCD Atik } \\
314 \mathrm{~L}+\end{array}$ \\
\hline
\end{tabular}




\begin{tabular}{|c|c|c|c|c|c|}
\hline Star & & & & & \\
\hline HJD-2400000 & Error & $\mathrm{I} / \mathrm{I}$ & Method & Points & Observer \\
\hline 57773.47005 & 0.0013 & II & $\mathrm{CCD}+\mathrm{V}$ & $318 / 228$ & Lehky M. \\
\hline 57774.27751 & 0.0009 & II & CCD + Clear & $460 / 79$ & Bílek F. \\
\hline 57774.47893 & 0.0009 & I & CCD + Clear & $460 / 291$ & Bílek F. \\
\hline 57775.28902 & 0.0012 & $\mathrm{I}$ & CCD + Clear & $532 / 96$ & Šmelcer L. \\
\hline 57775.49026 & 0.0013 & II & CCD + Clear & $532 / 317$ & Šmelcer L. \\
\hline 57776.29873 & 0.0009 & II & CCD + Clear & $424 / 92$ & Bílek F. \\
\hline 57776.50093 & 0.0008 & $\mathrm{I}$ & CCD + Clear & $424 / 280$ & Bílek F. \\
\hline 57798.31980 & 0.0007 & $\mathrm{I}$ & CCD + Clear & $382 / 86$ & Bílek F. \\
\hline 57799.33042 & 0.0007 & II & $\mathrm{CCD}+$ Clear & $395 / 97$ & Bílek F. \\
\hline 57800.34075 & 0.0006 & $\mathrm{I}$ & CCD + Clear & $387 / 106$ & Bílek F. \\
\hline 57801.35163 & 0.0008 & II & $\mathrm{CCD}+$ Clear & $227 / 118$ & Bílek F. \\
\hline 57829.43352 & 0.0011 & $\mathrm{I}$ & CCD + Clear & $321 / 171$ & Bílek F. \\
\hline 57838.32286 & 0.0015 & $\mathrm{I}$ & CCD + Clear & $124 / 57$ & Bílek F. \\
\hline 57991.46288 & 0.0009 & $\mathrm{I}$ & CCD + Clear & $262 / 100$ & Šmelcer L. \\
\hline 57995.50473 & 0.0007 & $\mathrm{I}$ & CCD + Clear & $304 / 206$ & Šmelcer L. \\
\hline 58027.42600 & 0.0008 & I & CCD + Clear & $251 / 135$ & Šmelcer L. \\
\hline
\end{tabular}

CzeV837 Cas 57642.48950

57656.31253

57658.61656

57725.42805

57739.25050

57755.37837
0.0001 I CCD+Clear 349/206 Bílek F.

0.0001 I CCD + Clear 303/65 Bílek F .

0.0002 I CCD + Clear 344/319 Bílek F.

0.0001 I CCD + Clear 521/236 Bílek F.

$0.0001 \mathrm{I} \quad \mathrm{CCD}+$ Clear $\quad 374 / 69 \quad$ Bílek F

0.0001 I CCD +Clear 468/185 Bílek F.
Technique

BlueEye600, RiLA RCT

$600 / 3000+$ MII G4-9000EC $+$

NWT 200/860 + CCD Atik $314 \mathrm{~L}+$

NWT 200/860 + CCD Atik $314 \mathrm{~L}+$

Celestron SCT 280/1765

MII G2-4000

Celestron SCT 280/1765 +

MII G2-4000

NWT 200/860 + CCD Atik $314 \mathrm{~L}+$

NWT 200/860 + CCD Atik $314 \mathrm{~L}+$

NWT 200/860 + CCD Atik $314 \mathrm{~L}+$

NWT 200/860 + CCD Atik $314 \mathrm{~L}+$

NWT 200/860 + CCD Atik $314 \mathrm{~L}+$

NWT 200/860 + CCD Atik $314 \mathrm{~L}+$

NWT 200/860 + CCD Atik $314 \mathrm{~L}+$

NWT 200/860 + CCD Atik $314 \mathrm{~L}+$

Celestron SCT 280/1765 +

MII G2-4000

Celestron SCT 280/1765 -

MII G2-4000

Celestron SCT 280/1765 + MII G2-4000

NWT 200/860 + CCD Atik $314 \mathrm{~L}+$

NWT 200/860 + CCD Atik $314 \mathrm{~L}+$

NWT 200/860 + CCD Atik $314 \mathrm{~L}+$

NWT 200/860 + CCD Atik $314 \mathrm{~L}+$

NWT 200/860 + CCD Atik $314 \mathrm{~L}+$

NWT 200/860 + CCD Atik $314 \mathrm{~L}+$ 


\begin{tabular}{|c|c|c|c|c|c|c|}
\hline \multirow{3}{*}{$\begin{array}{l}\text { Star } \\
\text { HJD-2400000 } \\
57764.59408\end{array}$} & \multirow{3}{*}{$\begin{array}{l}\text { Error } \\
0.0002\end{array}$} & \multirow{2}{*}{\multicolumn{2}{|c|}{ I/II Method }} & \multirow{3}{*}{$\begin{array}{l}\text { Points } \\
530 / 445\end{array}$} & \multirow{3}{*}{$\begin{array}{l}\text { Observer } \\
\text { Bílek F. }\end{array}$} & \multirow[b]{2}{*}{ Technique } \\
\hline & & & & & & \\
\hline & & I & CCD + Clear & & & $\begin{array}{l}\text { NWT 200/860 + CCD Atik } \\
314 \mathrm{~L}+\end{array}$ \\
\hline 57838.31743 & 0.0002 & I & $\mathrm{CCD}+$ Clear & $121 / 52$ & Bílek F. & $\begin{array}{l}\text { NWT } 200 / 860+\text { CCD Atik } \\
314 \mathrm{~L}+\end{array}$ \\
\hline 58043.35762 & 0.0001 & I & CCD+Clear & 409/134 & Bílek F. & $\begin{array}{l}\text { NWT } 200 / 860+\text { CCD Atik } \\
314 \mathrm{~L}+\end{array}$ \\
\hline 58073.30876 & 0.0001 & I & $\mathrm{CCD}+$ Clear & $248 / 117$ & Bílek F. & $\begin{array}{l}\text { NWT } 200 / 860+\text { CCD Atik } \\
314 \mathrm{~L}+\end{array}$ \\
\hline 58080.22018 & 0.0002 & I & CCD + Clear & $444 / 33$ & Bílek F. & $\begin{array}{l}\text { NWT 200/860 + CCD Atik } \\
314 \mathrm{~L}+\end{array}$ \\
\hline 58112.47425 & 0.0001 & I & CCD+Clear & $528 / 278$ & Bílek F. & $\begin{array}{l}\text { NWT 200/860 + CCD Atik } \\
314 \mathrm{~L}+\end{array}$ \\
\hline
\end{tabular}

EG Cas

58042.47607

EP Cas

57773.29032

GJ 3236 Cas 57627.59310

57628.36405

57631.44901

57632.60541

57633.37622

57634.53443

57639.54686

57640.31783

57644.56071

57645.33275

57646.48798

57656.51426

57658.44337
0.0005 I $\quad$ CCD + R 188/105 Lehky M.

0.0004 II CCD + Clear $\quad 313 / 159$ Bragagnolo U.

Sky-Watcher REF 100/900 + QHY9

HK25， NWT 250/1000 + SBIG ST-7 + BVRcIc

NWT 200/860 + CCD Atik $314 \mathrm{~L}+$

NWT 200/860 + CCD Atik $314 \mathrm{~L}+$

NWT 200/860 + CCD Atik $314 \mathrm{~L}+$

NWT 200/860 + CCD Atik $314 \mathrm{~L}+$

NWT 200/860 + CCD Atik $314 \mathrm{~L}+$

NWT 200/860 + CCD Atik $314 \mathrm{~L}+$

NWT 200/860 + CCD Atik $314 \mathrm{~L}+$

NWT 200/860 + CCD Atik $314 \mathrm{~L}+$

NWT 200/860 + CCD Atik $314 \mathrm{~L}+$

NWT 200/860 + CCD Atik $314 \mathrm{~L}+$

NWT 200/860 + CCD Atik $314 \mathrm{~L}+$

NWT 200/860 + CCD Atik $314 \mathrm{~L}+$

NWT 200/860 + CCD Atik $314 \mathrm{~L}+$ 


\begin{tabular}{|c|c|c|c|c|c|c|}
\hline \multicolumn{7}{|l|}{ Star } \\
\hline HJD-2400000 & Error & I/II & Method & Points & Observer & Technique \\
\hline 57661.52831 & 0.0001 & II & $\mathrm{CCD}+$ Clear & $345 / 271$ & Bílek F. & $\begin{array}{l}\text { NWT 200/860 + CCD Atik } \\
314 \mathrm{~L}+\end{array}$ \\
\hline 57714.35776 & 0.0002 & $\mathrm{I}$ & $\mathrm{CCD}+$ Clear & $190 / 147$ & Bílek F. & $\begin{array}{l}\text { NWT } 200 / 860+\text { CCD Atik } \\
314 \mathrm{~L}+\end{array}$ \\
\hline 57715.51734 & 0.0001 & I & $\mathrm{CCD}+\mathrm{R}$ & $379 / 235$ & Vrašták M & $\begin{array}{l}\text { NWT 240/1200+MII G2-1600, } \\
80 / 400+\text { G1-0300 }\end{array}$ \\
\hline 57722.45755 & 0.0002 & II & $\mathrm{CCD}+$ Clear & $461 / 254$ & Bílek F. & $\begin{array}{l}\text { NWT } 200 / 860+\text { CCD Atik } \\
314 \mathrm{~L}+\end{array}$ \\
\hline 57722.45787 & 0.0005 & II & $\mathrm{CCD}+\mathrm{R}$ & $386 / 330$ & Lehky M. & $\begin{array}{l}\text { BlueEye600, RiLA RCT } \\
600 / 3000+\text { MII G4-9000CE } \\
+\end{array}$ \\
\hline 57725.54262 & 0.0002 & II & $\mathrm{CCD}+$ Clear & $516 / 339$ & Bílek F. & $\begin{array}{l}\text { NWT } 200 / 860+\text { CCD Atik } \\
314 \mathrm{~L}+\end{array}$ \\
\hline 57726.31325 & 0.0002 & II & $\mathrm{CCD}+$ Clear & $410 / 127$ & Bílek F. & $\begin{array}{l}\text { NWT } 200 / 860+\text { CCD Atik } \\
314 \mathrm{~L}+\end{array}$ \\
\hline 57733.25487 & 0.0002 & II & $\mathrm{CCD}+$ Clear & $473 / 71$ & Bílek F. & $\begin{array}{l}\text { NWT } 200 / 860+\text { CCD Atik } \\
314 \mathrm{~L}+\end{array}$ \\
\hline 57739.42518 & 0.0002 & II & $\mathrm{CCD}+$ Clear & $377 / 232$ & Bílek F. & $\begin{array}{l}\text { NWT } 200 / 860+\text { CCD Atik } \\
314 \mathrm{~L}+\end{array}$ \\
\hline 57753.69393 & 0.0002 & I & $\mathrm{CCD}+$ Clear & $516 / 478$ & Bílek F. & $\begin{array}{l}\text { NWT } 200 / 860+\text { CCD Atik } \\
314 \mathrm{~L}+\end{array}$ \\
\hline 57755.23474 & 0.0001 & I & $\mathrm{CCD}+$ Clear & $464 / 51$ & Bílek F. & $\begin{array}{l}\text { NWT } 200 / 860+\text { CCD Atik } \\
314 \mathrm{~L}+\end{array}$ \\
\hline 57755.62123 & 0.0001 & II & $\mathrm{CCD}+$ Clear & $464 / 407$ & Bílek F. & $\begin{array}{l}\text { NWT } 200 / 860+\text { CCD Atik } \\
314 \mathrm{~L}+\end{array}$ \\
\hline 57764.48938 & 0.0002 & I & $\mathrm{CCD}+$ Clear & $520 / 310$ & Bílek F. & $\begin{array}{l}\text { NWT } 200 / 860+\text { CCD Atik } \\
314 \mathrm{~L}+\end{array}$ \\
\hline 57773.35818 & 0.0016 & II & $\mathrm{CCD}+\mathrm{V}$ & $349 / 152$ & Šmelcer L. & $\begin{array}{l}\text { Celestron SCT } 355 / 2460+ \\
\text { MII G2-1600 }\end{array}$ \\
\hline 57773.35894 & 0.0003 & II & $\mathrm{CCD}+\mathrm{V}$ & $316 / 134$ & Lehky M. & $\begin{array}{l}\text { BlueEye600, RiLA RCT } \\
600 / 3000+\text { MII G4-9000EC } \\
+\end{array}$ \\
\hline 57773.35999 & 0.0002 & II & $\mathrm{CCD}+$ Clear & $469 / 167$ & Bílek F. & $\begin{array}{l}\text { NWT } 200 / 860+\text { CCD Atik } \\
314 \mathrm{~L}+\end{array}$ \\
\hline 57774.51586 & 0.0002 & I & $\mathrm{CCD}+$ Clear & $466 / 331$ & Bílek F. & $\begin{array}{l}\text { NWT } 200 / 860+\text { CCD Atik } \\
314 \mathrm{~L}+\end{array}$ \\
\hline 57775.28714 & 0.0003 & I & $\mathrm{CCD}+\mathrm{V}$ & $196 / 87$ & Lomoz F. & Newton $300 / 1200+$ ST2000XM \\
\hline 57775.28732 & 0.0002 & I & $\mathrm{CCD}+$ Clear & $651 / 110$ & Šmelcer L. & $\begin{array}{l}\text { Celestron SCT 280/1765 + } \\
\text { MII G2-4000 }\end{array}$ \\
\hline 57775.28732 & 0.0001 & I & $\mathrm{CCD}+\mathrm{I}$ & $541 / 39$ & Šmelcer L. & $\begin{array}{l}\text { Celestron SCT } 355 / 2460+ \\
\text { MII G2-1600 }\end{array}$ \\
\hline 57775.28769 & 0.0001 & I & CCD + Clear & $472 / 96$ & Bílek F. & $\begin{array}{l}\text { NWT } 200 / 860+\text { CCD Atik } \\
314 \mathrm{~L}+\end{array}$ \\
\hline 57775.28806 & 0.0001 & I & $\mathrm{CCD}+$ Clear & $126 / 91$ & Lomoz F. & STN 254/1016+G2-8300 \\
\hline 57775.67383 & 0.0002 & II & $\mathrm{CCD}+\mathrm{I}$ & $541 / 489$ & Šmelcer L. & $\begin{array}{l}\text { Celestron SCT } 355 / 2460+ \\
\text { MII G2-1600 }\end{array}$ \\
\hline 57775.67401 & 0.0003 & II & $\mathrm{CCD}+$ Clear & $651 / 585$ & Šmelcer L. & $\begin{array}{l}\text { Celestron SCT 280/1765 + } \\
\text { MII G2-4000 }\end{array}$ \\
\hline
\end{tabular}




\begin{tabular}{|c|c|c|c|c|c|c|}
\hline $\begin{array}{l}\text { Star } \\
\text { HJD-2400000 }\end{array}$ & Error & $\mathrm{I} / \mathrm{I}$ & Method & Points & Observer & Technique \\
\hline 57776.44525 & 0.0001 & II & CCD + Clear & $415 / 225$ & Bílek F. & $\begin{array}{l}\text { NWT 200/860 + CCD Atik } \\
314 \mathrm{~L}+\end{array}$ \\
\hline 57798.42480 & 0.0002 & $\mathrm{I}$ & CCD + Clear & $397 / 189$ & Bílek F. & $\begin{array}{l}\text { NWT 200/860 + CCD Atik } \\
314 \mathrm{~L}+\end{array}$ \\
\hline 57799.58164 & 0.0006 & II & CCD + Clear & $402 / 337$ & Bílek F. & $\begin{array}{l}\text { NWT } 200 / 860+\text { CCD Atik } \\
314 \mathrm{~L}+\end{array}$ \\
\hline 57800.35439 & 0.0002 & II & CCD + Clear & $395 / 121$ & Bílek F. & $\begin{array}{l}\text { NWT 200/860 + CCD Atik } \\
314 \mathrm{~L}+\end{array}$ \\
\hline 57812.30800 & 0.0001 & $\mathrm{I}$ & CCD + Clear & $246 / 69$ & Bílek F. & $\begin{array}{l}\text { NWT 200/860 + CCD Atik } \\
314 \mathrm{~L}+\end{array}$ \\
\hline 57829.27542 & 0.0002 & $\mathrm{I}$ & CCD + Clear & $334 / 24$ & Bílek F. & $\begin{array}{l}\text { NWT 200/860 + CCD Atik } \\
314 \mathrm{~L}+\end{array}$ \\
\hline 57994.32503 & 0.0003 & $\mathrm{I}$ & CCD + Clear & $320 / 37$ & Bílek F. & $\begin{array}{l}\text { NWT 200/860 + CCD Atik } \\
314 \mathrm{~L}+\end{array}$ \\
\hline 57995.48175 & 0.0002 & II & CCD + Clear & $323 / 185$ & Bílek F. & $\begin{array}{l}\text { NWT 200/860 + CCD Atik } \\
314 \mathrm{~L}+\end{array}$ \\
\hline 57995.48192 & 0.0002 & II & CCD + Clear & $350 / 197$ & Šmelcer L. & $\begin{array}{l}\text { Celestron SCT } 280 / 1765+ \\
\text { MII G2-4000 }\end{array}$ \\
\hline 58005.50807 & 0.0004 & II & CCD + Clear & $349 / 227$ & Bílek F. & $\begin{array}{l}\text { NWT 200/860 + CCD Atik } \\
314 \mathrm{~L}+\end{array}$ \\
\hline 58026.33229 & 0.0002 & II & CCD + Clear & $400 / 92$ & Bílek F. & $\begin{array}{l}\text { NWT 200/860 + CCD Atik } \\
314 \mathrm{~L}+\end{array}$ \\
\hline 58027.48843 & 0.0001 & $\mathrm{I}$ & CCD + Clear & $404 / 237$ & Bílek F. & $\begin{array}{l}\text { NWT } 200 / 860+\text { CCD Atik } \\
314 \mathrm{~L}+\end{array}$ \\
\hline 58027.48916 & 0.0002 & $\mathrm{I}$ & CCD + Clear & $332 / 258$ & Šmelcer L. & $\begin{array}{l}\text { Celestron SCT } 280 / 1765+ \\
\text { MII G2-4000 }\end{array}$ \\
\hline 58043.29944 & 0.0002 & II & CCD + Clear & $409 / 80$ & Bílek F. & $\begin{array}{l}\text { NWT 200/860 + CCD Atik } \\
314 \mathrm{~L}+\end{array}$ \\
\hline 58043.30021 & 0.0002 & II & CCD + Clear & $394 / 117$ & Šmelcer L. & $\begin{array}{l}\text { Celestron SCT } 280 / 1765+ \\
\text { MII G2-4000 }\end{array}$ \\
\hline 58046.38482 & 0.0002 & II & CCD + Clear & $379 / 165$ & Bílek F. & $\begin{array}{l}\text { NWT 200/860 + CCD Atik } \\
314 \mathrm{~L}+\end{array}$ \\
\hline 58061.42434 & 0.0005 & $\mathrm{I}$ & CCD + Clear & $475 / 217$ & Bílek F. & $\begin{array}{l}\text { NWT 200/860 + CCD Atik } \\
314 \mathrm{~L}+\end{array}$ \\
\hline 58073.37865 & 0.0002 & II & CCD + Clear & $238 / 182$ & Bílek F. & $\begin{array}{l}\text { NWT 200/860 + CCD Atik } \\
314 \mathrm{~L}+\end{array}$ \\
\hline 58080.32014 & 0.0001 & II & CCD + Clear & $442 / 125$ & Bílek F. & $\begin{array}{l}\text { NWT 200/860 + CCD Atik } \\
314 \mathrm{~L}+\end{array}$ \\
\hline 58081.47651 & 0.0002 & $\mathrm{I}$ & CCD + Clear & $457 / 271$ & Bílek F. & $\begin{array}{l}\text { NWT 200/860 + CCD Atik } \\
314 \mathrm{~L}+\end{array}$ \\
\hline 58094.20298 & 0.0003 & II & CCD + Clear & $485 / 26$ & Bílek F. & $\begin{array}{l}\text { NWT 200/860 + CCD Atik } \\
314 \mathrm{~L}+\end{array}$ \\
\hline 58094.58753 & 0.0002 & $\mathrm{I}$ & CCD + Clear & $485 / 386$ & Bílek F. & $\begin{array}{l}\text { NWT 200/860 + CCD Atik } \\
314 \mathrm{~L}+\end{array}$ \\
\hline 58095.35821 & 0.0003 & $\mathrm{I}$ & $\mathrm{CCD}+\mathrm{V}$ & $185 / 100$ & Šmelcer L. & $\begin{array}{l}\text { Celestron SCT } 355 / 2460+ \\
\text { MII G2-1600 }\end{array}$ \\
\hline 58095.35845 & 0.0003 & $\mathrm{I}$ & CCD + Clear & $366 / 150$ & Bílek F. & $\begin{array}{l}\text { NWT 200/860 }+ \text { CCD Atik } \\
314 \mathrm{~L}+\end{array}$ \\
\hline
\end{tabular}




\begin{tabular}{|c|c|c|c|c|c|c|}
\hline \multirow{3}{*}{$\begin{array}{l}\text { Star } \\
\text { HJD-2400000 } \\
58112.32607\end{array}$} & \multirow{3}{*}{$\begin{array}{l}\text { Error } \\
0.0001\end{array}$} & \multirow{2}{*}{\multicolumn{2}{|c|}{ I/II Method }} & \multirow{3}{*}{$\begin{array}{l}\text { Points } \\
528 / 140\end{array}$} & \multirow{3}{*}{$\begin{array}{l}\text { Observer } \\
\text { Bílek F. }\end{array}$} & \multirow[b]{2}{*}{ Technique } \\
\hline & & & & & & \\
\hline & & I & $\mathrm{CCD}+$ Clear & & & $\begin{array}{l}\text { NWT 200/860 + CCD Atik } \\
314 \mathrm{~L}+\end{array}$ \\
\hline 58112.71239 & 0.0005 & II & CCD + Clear & $528 / 499$ & Bílek F. & $\begin{array}{l}\text { NWT 200/860 + CCD Atik } \\
\text { 314L+ }\end{array}$ \\
\hline 58113.48058 & 0.0003 & II & $\mathrm{CCD}+\mathrm{V}$ & $389 / 256$ & Šmelcer L. & $\begin{array}{l}\text { Celestron SCT } 355 / 2460+ \\
\text { MII G2-1600 }\end{array}$ \\
\hline 58113.48397 & 0.0002 & II & CCD + Clear & $510 / 288$ & Bílek F. & $\begin{array}{l}\text { NWT } 200 / 860+\text { CCD Atik } \\
314 \mathrm{~L}+\end{array}$ \\
\hline 58134.30814 & 0.0002 & II & CCD + Clear & $141 / 97$ & Bílek F. & $\begin{array}{l}\text { NWT } 200 / 860+\text { CCD Atik } \\
314 \mathrm{~L}+\end{array}$ \\
\hline 58149.34652 & 0.0002 & I & CCD + Clear & $360 / 99$ & Šmelcer L. & $\begin{array}{l}\text { Celestron SCT } 280 / 1765+ \\
\text { MII G2- } 4000\end{array}$ \\
\hline 58175.57009 & 0.0002 & I & CCD + Clear & $422 / 319$ & Bílek F. & $\begin{array}{l}\text { NWT } 200 / 860+\text { CCD Atik } \\
314 \mathrm{~L}+\end{array}$ \\
\hline 58178.26960 & 0.0002 & II & CCD + Clear & $424 / 33$ & Bílek F. & $\begin{array}{l}\text { NWT } 200 / 860+\text { CCD Atik } \\
314 \mathrm{~L}+\end{array}$ \\
\hline 58178.26987 & 0.0003 & I & CCD + Clear & $455 / 46$ & Šmelcer L. & $\begin{array}{l}\text { Celestron SCT 280/1765 + } \\
\text { MII G2-4000 }\end{array}$ \\
\hline 58179.42543 & 0.0004 & I & CCD + Clear & $259 / 199$ & Šmelcer L. & $\begin{array}{l}\text { Celestron SCT 280/1765 + } \\
\text { MII G2-4000 }\end{array}$ \\
\hline
\end{tabular}

IR Cas

57989.54150

58121.25442

IT Cas

57726.21323

MT Cas

58005.51313

MW Cas

58005.51884

0.0006 I $\quad C C D+$ Clear $\quad 162 / 76 \quad$ Urbaník M.

0.0003 II $\quad$ CCD + Clear $\quad 189 / 34 \quad$ Bragagnolo U.

$0.0001 \mathrm{I} \quad \mathrm{CCD}+\mathrm{V} \quad 186 / 60 \quad$ Magris $\mathrm{M}$.

0.0001 I $\mathrm{CCD}+$ Clear $86 / 50 \quad$ Červinka L.

NWT 200/1000 + Atik 314L +

0.0002 I CCD+Clear 175/72 Urbaník M.

Sky-Watcher NWT 150/750 + MII G2-8300

NSVS 3686195 Cas

57974.45236

57977.39007

0.0014 I DSLR

0.0008 II DSLR

NT Cas

57984.35566
0.0003 I CCD + Clear $\quad 180 / 45$ Urbaník M.
Sky-Watcher REF 100/900 + QHY9

REF $120 / 600+$ Orion 3G $\mathrm{CCD}+0.5 \mathrm{x}$ reducer

Sky-Watcher NWT 150/750 + MII G2-8300
$74 / 44 \quad$ Sergey I.

$47 / 26 \quad$ Sergey I.
Newton 200 mm (1:5) + Canon 400D

Newton 200 mm (1:5) + Canon 400D 
Star

HJD-2400000

Error I/II Method

Points Observer

Technique

OR Cas

58076.37998

QQ Cas

57764.35162

RZ Cas

58045.35243

SERIV 19 Cas

57729.32120

V0337 Cas

57938.41959

V0359 Cas

57646.34730

V0366 Cas

57775.28571

57956.51949

57980.58644

57980.58651

57980.58652

V0375 Cas

58023.64444

V0381 Cas

57705.46097
0.0001 I CCD + Clear 221/88 Bragagnolo U.

Sky-Watcher REF 100/900 + QHY9

$0.0002 \mathrm{I} \quad \mathrm{CCD}+\mathrm{R} \quad 514 / 182$ Šmelcer L.

Celestron SCT 355/2460 + MII G2-1600

Pentacon $1.8 / 50+$ MII G2$1600,80 / 400+\mathrm{G} 1-0$

Newton $200 \mathrm{~mm}(1: 5)+$ Canon 400D

Sky-Watcher NWT 150/750 + MII G2-8300

0.0003 I $\quad$ CCD + Clear $100 / 65$ Červinka L. R102/500 + Atik 314L +

0.0002 I CCD +Clear 190/129 Bragagnolo U. Sky-Watcher REF 100/900 QHY9

0.0001 II CCD +Clear 184/141 Vrašták M.

NWT 240/1200+MII G2-1600, $80 / 400+$ G1-0300

0.0003 II $\quad$ CCD $+\mathrm{I} \quad 48 / 37 \quad$ Lehky M.

JST, NWT 400/2000 + MII

G2-1600 + BVRcIc

0.0003 II $\mathrm{CCD}+\mathrm{V} \quad 46 / 37 \quad$ Lehky M.

JST, NWT 400/2000 + MII

G2-1600 + BVRcIc

0.0002 II $\quad \mathrm{CCD}+\mathrm{R} \quad 48 / 37 \quad$ Lehky $\mathrm{M}$.

JST, NWT 400/2000 + MII

G2-1600 + BVRcIc
Sky-Watcher REF 100/900 + QHY9

0.0004 I CCD + Clear 150/72 Bragagnolo U. Sky-Watcher REF 100/900 + QHY9 


\begin{tabular}{|c|c|c|c|c|c|}
\hline $\begin{array}{l}\text { Star } \\
\text { HJD-2400000 }\end{array}$ & Error & \multicolumn{2}{|c|}{ I/II Method } & Points & Observer \\
\hline $\begin{array}{l}\text { V0445 Cas } \\
58027.34270\end{array}$ & 0.0004 & I & $\mathrm{CCD}+\mathrm{R}$ & $72 / 29$ & Lehky M. \\
\hline 58027.34384 & 0.0006 & $\mathrm{I}$ & $\mathrm{CCD}+\mathrm{V}$ & $75 / 33$ & Lehky M. \\
\hline 58027.34535 & 0.0008 & I & $\mathrm{CCD}+\mathrm{I}$ & $75 / 31$ & Lehky M. \\
\hline $\begin{array}{l}\text { V0523 Cas } \\
58137.28975\end{array}$ & 0.0001 & I & $\mathrm{CCD}+\mathrm{I}$ & $81 / 53$ & Walter F. \\
\hline 58137.28980 & 0.0001 & $\mathrm{I}$ & $\mathrm{CCD}+\mathrm{R}$ & $102 / 77$ & Walter F. \\
\hline 58137.28982 & 0 & I & $\mathrm{CCD}+\mathrm{Clear}$ & $106 / 77$ & Walter F. \\
\hline
\end{tabular}

V0541 Cas 58091.50601

V0952 Cas 57995.37147 V0959 Cas 57735.30962 V1007 Cas 57966.49886 57966.49890 57966.49953 57996.54734 58025.43095 58025.43101 58025.43153 58052.31992 58052.32006 0.0004 I CCD + Clear 202/134 Lehky M.

0.0001 I CCD +Clear 252/111 Vrašták M.

NWT 240/1200+MII G2-1600, $80 / 400+$ G1-0300

HK25, NWT 250/1000 + SBIG ST-7 + Rc

ORION, NWT 500/1500 + MII G2 402

ORION, NWT 500/1500 + MII G2 402

ORION, NWT 500/1500 + MII G2 402

Sky-Watcher REF 100/900 + QHY9

ORION, NWT 500/1500 + MII G2 402

ORION, NWT 500/1500+ MII G2 402

ORION, NWT 500/1500 + MII G2 402

MARK SCT 406/4060, SBIG ST10XME

MARK SCT 406/4060, SBIG ST10XME 


\begin{tabular}{|c|c|c|c|c|c|}
\hline Star & & & & & \\
\hline HJD-2400000 & Error & I/II Method & Points & Observer & Technique \\
\hline 58052.32056 & 0.0003 & I $\quad \mathrm{CCD}+\mathrm{R}$ & $52 / 41$ & Walter F. & $\begin{array}{l}\text { MARK SCT } 406 / 4060, \text { SBIG } \\
\text { ST10XME }\end{array}$ \\
\hline
\end{tabular}

V1030 Cas 57979.37633

57979.37637

57979.37668

58044.32339

58044.32352

V1060 Cas

57966.53682

57966.53751

57966.53851

V1063 Cas

58058.46235

V1069 Cas

58024.35147

58024.55363

V1070 Cas 57984.46336

V1094 Cas 57811.33792

57811.33821

57811.59519

58028.37056

$\begin{array}{lllll}0.0002 & \text { I } & \text { CCD }+V & 104 / 57 & \text { Mazanec J. } \\ 0.0003 \text { I } & \text { CCD }+ \text { I } & 102 / 54 & \text { Mazanec J. } \\ 0.0001 & \text { I } & \text { CCD }+R & 108 / 60 & \text { Mazanec J. } \\ 0.0002 & \text { II } & \text { CCD }+V & 77 / 46 & \text { Vrašták M. } \\ 0.0002 \text { II } & \text { CCD }+R & 79 / 47 & \text { Vrašták M. } \\ & & & & \\ 0.0005 \text { I } & \text { CCD }+V & 50 / 24 & \text { Lehky M. } \\ 0.0004 \text { I } & \text { CCD }+R & 52 / 24 & \text { Lehky M. } \\ 0.0005 \text { I } & \text { CCD }+\mathrm{I} & 50 / 22 & \text { Lehky M. }\end{array}$

0.0003 I CCD + Clear 303/51 Bragagnolo U.

Sky-Watcher REF 100/900 QHY9

ORION, NWT 500/1500 + MII G2 402

ORION, NWT 500/1500 + MII G2 402

ORION, NWT 500/1500 + MII G2 402

NWT 355/1600+MII G2-1600, 80/400+G1-0301

NWT 355/1600+MII G2-1600, 80/400+G1-0301

HK25, NWT 250/1000 + SBIG ST-7 + BVRcIc

HK25， NWT 250/1000 SBIG ST-7 + BVRcIc HK25, NWT 250/1000 SBIG ST-7 + BVRcIc

DATEL, NWT 200/800 + MII G2 8300

DATEL, NWT 200/800 + MII G2 8300

NWT 240/1200+MII G2-1600, 80/400+G1-0300

Set sekce, NWT 150/750 + G2-1600

Set sekce, NWT $150 / 750+$ G2-1600

Set sekce, NWT 150/750 + G2-1600

NWT 150/600 + Canon 20D +

MPCC mk.III 


\begin{tabular}{|c|c|c|c|c|c|}
\hline $\begin{array}{l}\text { Star } \\
\text { HJD-2400000 }\end{array}$ & Error & $\mathrm{I} / \mathrm{II}$ & Method & Points & Observer \\
\hline $\begin{array}{l}\text { V1106 Cas } \\
58095.24934\end{array}$ & 0.0003 & I & $\mathrm{CCD}+\mathrm{R}$ & $79 / 37$ & Lehky M. \\
\hline $\begin{array}{l}\text { V1112 Cas } \\
57750.52206\end{array}$ & 0.0007 & I & CCD + Clear & $187 / 69$ & Bragagnolo \\
\hline 57780.30834 & 0.0003 & I & $\mathrm{CCD}+$ Clear & $134 / 57$ & Lehky M. \\
\hline 57997.45940 & 0.0002 & I & $\mathrm{CCD}+\mathrm{V}$ & $39 / 19$ & Šuchaň J. \\
\hline $\begin{array}{l}\text { V1175 Cas } \\
58043.34177\end{array}$ & 0.0004 & $\mathrm{I}$ & $\mathrm{CCD}+\mathrm{R}$ & $78 / 56$ & Lehky M. \\
\hline 58043.34519 & 0.0009 & $\mathrm{I}$ & $\mathrm{CCD}+\mathrm{V}$ & $54 / 45$ & Lehky M. \\
\hline 58043.34596 & 0.0007 & I & $\mathrm{CCD}+\mathrm{I}$ & $68 / 41$ & Lehky M. \\
\hline $\begin{array}{l}\text { V1179 Cas } \\
57715.48870\end{array}$ & 0.0001 & I & $\mathrm{CCD}+\mathrm{R}$ & 289/192 & Vrašták M \\
\hline 58043.37722 & 0.0013 & II & CCD + Clear & $338 / 168$ & Šmelcer L. \\
\hline 58144.33539 & 0.0002 & I & $\mathrm{CCD}+\mathrm{R}$ & $422 / 150$ & Šmelcer L. \\
\hline 58149.34161 & 0.0001 & I & CCD + Clear & $443 / 126$ & Šmelcer L. \\
\hline 58178.53979 & 0.0026 & II & CCD + Clear & $367 / 295$ & Šmelcer L. \\
\hline 58179.37704 & 0.0003 & I & CCD + Clear & $219 / 158$ & Šmelcer L. \\
\hline
\end{tabular}

V1261 Cas 58076.42442

0.0003 II CCD + Clear 215/172 Bragagnolo U.

NWT 240/1200+MII G2-1600, 80/400+G1-0300

Celestron SCT 280/1765 + MII G2-4000

Celestron SCT $355 / 2460+$ MII G2-1600

Celestron SCT 280/1765 + MII G2-4000

Celestron SCT 280/1765 + MII G2-4000

Celestron SCT 280/1765 + MII G2-4000

Sky-Watcher REF 100/900 QHY9

VSX J235957.2+513349 Cas

57969.38982 0.0004 I $\quad$ CCD + Clear $\quad 131 / 39 \quad$ Tylšar M. NWT250/1000,G2-8300

ZZ Cas

58050.64801

0.0003 I CCD+Clear 309/186 Bragagnolo U.

Sky-Watcher REF 100/900 + QHY9 


\begin{tabular}{|c|c|c|c|c|c|}
\hline Star & & & & & \\
\hline HJD-2400000 & Error & I/II Method & Points & Observer & Technique \\
\hline ASAS J11334 & & & & & \\
\hline 57753.78998 & 0.0009 & I $\quad \mathrm{CCD}+\mathrm{R}$ & $26 / 21$ & Mašek M. & $\begin{array}{l}\text { FRAM, Nikkor LNS } 106 / 300 \\
+ \text { G4-16000 }\end{array}$ \\
\hline
\end{tabular}

ASAS J113728-5235.3 Cen
57753.74424
$0.0015 \mathrm{I} \quad \mathrm{CCD}+\mathrm{R} \quad 28 / 15 \quad$ Mašek M.
FRAM, Nikkor LNS 106/300 + G4-16000

ASAS J113941-5412.5 Cen

\begin{tabular}{|c|c|c|c|c|c|c|}
\hline 57753.71768 & 0.0014 & I & $\mathrm{CCD}+\mathrm{R}$ & $29 / 12$ & Mašek M. & $\begin{array}{l}\text { FRAM, Nikkor LNS } 106 / 300 \\
+ \text { G4- } 16000\end{array}$ \\
\hline \multicolumn{7}{|c|}{ ASAS J114914-5447.6 Cen } \\
\hline 57753.70882 & 0.0005 & I & $\mathrm{CCD}+\mathrm{R}$ & $28 / 10$ & Mašek M. & $\begin{array}{l}\text { FRAM, Nikkor LNS } 106 / 300 \\
+ \text { G4- } 16000\end{array}$ \\
\hline \multicolumn{7}{|l|}{ V0508 Cen } \\
\hline 57872.67855 & 0 & II & $\mathrm{CCD}+$ Clear & $1310 /:$ & 9 Durantini L. & $\begin{array}{l}\text { Celestron, SCT, } 350 / 3572+ \\
\text { SBIG ST- } 7+\text { reducer } 0\end{array}$ \\
\hline 57872.87528 & 0.0001 & I & $\mathrm{CCD}+$ Clear & $1310 /$ & 9Durantini L. & $\begin{array}{l}\text { Celestron, SCT, } 350 / 3572+ \\
\text { SBIG ST- } 7+\text { reducer } 0\end{array}$ \\
\hline
\end{tabular}

V0637 Cen

57830.68770

0.0002 I $\quad$ CCD + Clear $\quad 146 / 80 \quad$ Tornatore M.

Schmidt-Cassegrain $14+$ CCD Sbig ST7 + Reductor

NSVS 3325547 Cep

57885.44732

0.0005 I DSLR

123/72 Sergey I.

Newton 200 mm (1:5) + Canon 400D

2MASS J21545261+6245282 Cep

57967.40964

0.0001 I CCD+Clear 287/111 Urbaník M.

Sky-Watcher NWT 150/750 + MII G2-8300

CzeV1098 Cep

57590.51864

0.0013 I DSLR

0.0015 II DSLR

169/134 Walter F.

160/39 Walter F.

NWT 150/750 + Canon 350 D

57607.40861

0.0017 I DSLR

141/40 Walter F.

Skywatcher REF 102/600 +

Canon $350 \mathrm{~d}$

57608.46840

0.0017 II DSLR

159/118 Walter F.

Skywatcher REF 102/600 +

Canon $350 \mathrm{~d}$

57627.49592

0.0011 II DSLR

157/62 Walter F.

Tamron SP LNS 62.5/500 + Canon $450 \mathrm{D}$

57639.46870

0.0011 II DSLR $\quad 157 / 62 \quad$ Walter F.

Skywatcher REF 102/600

Canon $450 \mathrm{~d}$ 


\begin{tabular}{|c|c|c|c|c|c|}
\hline Star & & & & & \\
\hline HJD-2400000 & Error & I/II Method & Points & Observer & Technique \\
\hline 57725.40377 & 0.0019 & II $\quad$ DSLR & $105 / 64$ & Walter F. & $\begin{array}{l}\text { Skywatcher REF } 102 / 600 \text {, Ca- } \\
\text { non } 450 \mathrm{D}\end{array}$ \\
\hline
\end{tabular}

CzeV1234 Cep

58004.48469

58008.59162

CzeV153 Cep

57966.43270

57974.51058

CzeV821 Cep

57725.25019

CzeV880 Cep

57725.50430

GW Cep

57853.45933

LP Cep

58022.31562

LY Cep

57967.38853

NSVS 156985 Cep

57893.41696

0.0005 I DSLR

94/31 Sergey I.

0.0001 I $\quad \mathrm{CCD}+\mathrm{R} \quad 325 / 166$ Šmelcer L.

Celestron SCT 355/2460 + MII G2-1600

Skywatcher REF 102/600 + Canon 450 D

Sky-Watcher NWT 150/750 + MII G2-8300

Sky-Watcher NWT 150/750 + MII G2-8300

Skywatcher REF 102/600 + Canon 450 D

49/18 Sergey I.

Newton 200 mm (1:5) + Canon 400D

0.0007 I CCD+Clear 286/79 Urbaník M.

Sky-Watcher NWT 150/750 + MII G2-8300

NSVS 3259747 Cep

57984.48637

0.0006 I DSLR

160/61 Walter F.

SERIV 121 Cep

58127.33164

0.0012 I DSLR

95/82 Sergey I.

58134.35826
LNS $58 / 200+$ Canon 350 D

Newton $200 \mathrm{~mm}(1: 5)+$ Canon 400D

Newton $200 \mathrm{~mm}(1: 5)+$ Canon 400D

$$
\text { Newton } 200 \mathrm{~mm}(1: 5)+\text { Canon }
$$
400D 


\begin{tabular}{|c|c|c|c|c|c|}
\hline Star & & & & & \\
\hline HJD-2400000 & Error & I/II Method & Points & Observer & Technique \\
\hline 58142.33971 & 0.0022 & I $\quad$ DSLR & $67 / 45$ & Sergey I. & $\begin{array}{l}\text { Newton } 200 \mathrm{~mm}(1: 5)+\text { Canon } \\
\text { 400D }\end{array}$ \\
\hline
\end{tabular}

SU Cep

57725.32669

U Cep

57853.45876

V0397 Cep

58044.27678

V0489 Cep

57508.51876

57517.45774

V0699 Cep

57723.28195

V0736 Cep

57722.26612

57983.45395

V0737 Cep

57887.38537

57888.43405

58062.45774

V0744 Cep
57750.24209

0.0003 II DSLR $\quad 148 / 51 \quad$ Walter F.

0.0008 II DSLR

832/394 Školník V.

0.0001 I CCD+Clear 228/87 Vrašták M.

0.0003 II CCD + Clear 222/125 Trnka J.

0.0005 I $\quad$ CCD + Clear 236/120 Trnka J.

0.0007 I $\quad C C D+$ Clear 155/87 Bragagnolo U.

Sky-Watcher REF 100/900 + QHY9

Pentacon $\quad 1.8 / 50+\mathrm{MII} \quad$ G2$1600,80 / 400+$ G1-0

Skywatcher NWT 200/1000 + SBIG ST-9E + comacorre Skywatcher NWT 200/1000+

SBIG ST-9E + comacorre

Canon $450 \mathrm{D}$

Tamron LNS 44/300 + Canon $600 \mathrm{D}$

Sky-Watcher REF 100/900 + QHY9

Tamron SP LNS 62.5/500+ Canon $350 \mathrm{D}$

0.0002 II DSLR

104/25 Sergey I.

111/60 Sergey I.

68/49 Gudmundsson

S.

Newton 200 mm (1:5) + Canon 400D

Newton $200 \mathrm{~mm}(1: 5)$ + Canon 400D

$400 \mathrm{~mm}$ LX200 SCT/SBIG STL11k CCD
0.0007 I $\quad$ CCD + Clear $\quad 175 / 39 \quad$ Bragagnolo U. Sky-Watcher REF 100/900 + QHY9

DATEL, NWT 200/800 + MII G2 8300 


\begin{tabular}{|c|c|c|c|c|c|c|}
\hline $\begin{array}{l}\text { Star } \\
\text { HJD-2400000 }\end{array}$ & Error & & Method & Points & Observer & Technique \\
\hline $\begin{array}{l}\text { V0796 Cep } \\
57853.50534\end{array}$ & 0.0002 & I & $\mathrm{CCD}+\mathrm{R}$ & $302 / 216$ & Šmelcer L. & $\begin{array}{l}\text { Celestron SCT } 355 / 2460+ \\
\text { MII G2-1600 }\end{array}$ \\
\hline $\begin{array}{l}\text { V0797 Cep } \\
57853.41951\end{array}$ & 0.0005 & II & $\mathrm{CCD}+\mathrm{R}$ & $271 / 103$ & Šmelcer L. & $\begin{array}{l}\text { Celestron SCT } 355 / 2460+ \\
\text { MII G2-1600 }\end{array}$ \\
\hline 57853.54630 & 0.0007 & I & $\mathrm{CCD}+\mathrm{R}$ & $271 / 210$ & Šmelcer L. & $\begin{array}{l}\text { Celestron SCT } 355 / 2460+ \\
\text { MII G2-1600 }\end{array}$ \\
\hline $\begin{array}{l}\text { V0808 Cep } \\
57779.60836\end{array}$ & 0.0003 & I & CCD + Clear & $294 / 145$ & Bragagnolo U. & $\begin{array}{l}\text { Sky-Watcher REF } 100 / 900+ \\
\text { QHY9 }\end{array}$ \\
\hline $\begin{array}{l}\text { V0817 Cep } \\
57997.45193\end{array}$ & 0.0004 & I & $\mathrm{CCD}+\mathrm{R}$ & $166 / 68$ & Vrašták M & $\begin{array}{l}\text { NWT 355/1600+MII G2-1600, } \\
80 / 400+\text { G1-0301 }\end{array}$ \\
\hline $\begin{array}{l}\text { V0830 Cep } \\
57915.42166\end{array}$ & 0.0003 & I & $\mathrm{CCD}+\mathrm{R}$ & $112 / 46$ & Hanžl D. & DATEL, NWT 200/800 + MII \\
\hline 57917.37338 & 0.0002 & I & $\mathrm{CCD}+\mathrm{R}$ & $146 / 20$ & Hanžl D. & $\begin{array}{l}\text { DATEL, NWT } 200 / 800+\text { MII } \\
\text { G2 } 8300\end{array}$ \\
\hline 57917.50343 & 0.0002 & II & $\mathrm{CCD}+\mathrm{R}$ & $146 / 103$ & Hanžl D. & $\begin{array}{l}\text { DATEL, NWT } 200 / 800+\text { MII } \\
\text { G2 } 8300\end{array}$ \\
\hline 57918.41393 & 0.0002 & II & $\mathrm{CCD}+\mathrm{R}$ & $126 / 22$ & Hanžl D. & $\begin{array}{l}\text { DATEL, NWT } 200 / 800+\text { MII } \\
\text { G2 } 8300\end{array}$ \\
\hline 57918.54329 & 0.0002 & I & $\mathrm{CCD}+\mathrm{R}$ & $126 / 110$ & Hanžl D. & $\begin{array}{l}\text { DATEL, NWT } 200 / 800+\text { MII } \\
\text { G2 } 8300\end{array}$ \\
\hline 57919.45465 & 0.0002 & I & $\mathrm{CCD}+\mathrm{R}$ & $139 / 67$ & Hanžl D. & $\begin{array}{l}\text { DATEL, NWT } 200 / 800+\text { MII } \\
\text { G2 } 8300\end{array}$ \\
\hline
\end{tabular}

V0835 Cep 57764.42687

V0854 Cep 57906.38586

V0855 Cep 58028.30785 58028.30790 58028.30794
0.0004 I $\quad$ CCD + Clear $\quad 283 / 141$ Walter F.

$0.0001 \mathrm{I} \quad \mathrm{CCD}+\mathrm{R} \quad 150 / 31 \quad$ Hanžl D.

$0.0002 \mathrm{I} \quad \mathrm{CCD}+\mathrm{R} \quad 128 / 72 \quad$ Mazanec J.

112/60 Mazanec J.

114/65 Mazanec J.
RL MARK 40/406, SBIG ST10XME

DATEL, NWT 200/800 + MII G2 8300

ORION, NWT 500/1500 + MII G2 402

ORION, NWT 500/1500 + MII G2 402

ORION, NWT 500/1500 + MII G2 402 


\section{Star \\ HJD-2400000 \\ V0883 Cep}

57968.39969

V0889 Cep

57994.37272

V0914 Cep

57980.46150

57981.42538

V0915 Cep

57667.40864

V0923 Cep

58001.49771

58001.49777

58001.49957

V0930 Cep

57964.38828

V0936 Cep

57735.43884

57735.43914

57735.43986

57751.27616

57751.27637

57751.27664

58005.35504
0.0002 I CCD + Clear $\quad 187 / 64$ Urbaník M.

$0.0003 \mathrm{I} \quad \mathrm{CCD}+\mathrm{R} \quad 133 / 54 \quad$ Hanžl D.

0.0003 II $\mathrm{CCD}+\mathrm{R} \quad 122 / 44 \quad$ Hanžl D.

$0.0004 \mathrm{I} \quad \mathrm{CCD}+\mathrm{R} \quad 71 / 19 \quad$ Hanžl D.

0.0003 I CCD +Clear 205/166 Jacobsen J.

0.0007 I DSLR 223/99 Walter F.

0.0008 I DSLR $\quad 211 / 96$ Walter F.

0.0003 I $\quad$ CCD + Clear $225 / 92$ Walter F.

0.0001 II CCD + Clear $\quad 159 / 75 \quad$ Vrašták M

$0.0003 \mathrm{I} \quad \mathrm{CCD}+\mathrm{V} \quad 300 / 228$ Mazanec J.

$0.0002 \mathrm{I} \quad \mathrm{CCD}+\mathrm{R} \quad 299 / 228$ Mazanec J.

$0.0004 \mathrm{I} \quad \mathrm{CCD}+\mathrm{I} \quad 295 / 225$ Mazanec J.

0.0004 II $\quad \mathrm{CCD}+\mathrm{I} \quad 236 / 127$ Mazanec J.

0.0002 II $\quad \mathrm{CCD}+\mathrm{R} \quad 236 / 127$ Mazanec J.

0.0003 II $\mathrm{CCD}+\mathrm{V} \quad 234 / 126$ Mazanec J.

0.0008 II $\mathrm{CCD}+\mathrm{R}$
172/16 Hanžl D.
Technique

Sky-Watcher NWT 150/750 + MII G2-8300

DATEL, NWT 200/800 + MII G2 8300

DATEL, NWT 200/800 + MII G2 8300

DATEL, NWT 200/800 + MII

G2 8300

EQ6, $80 \mathrm{~mm}$ refractor, SXVRH16

CZ Mirotar LNS 180/1000 Canon 350D

CZ Mirotar LNS 180/1000 + Canon 350D

CZ Kometensucher REF 200/1370 + SBIG ST7

NWT 240/1200+MII G2-1600, 80/400+G1-0300

ORION, NWT 500/1500

MII G2 402

ORION, NWT 500/1500+

MII G2 402

ORION, NWT 500/1500 + MII G2 402

ORION, NWT 500/1500 + MII G2 402

ORION, NWT 500/1500+ MII G2 402

ORION, NWT 500/1500 + MII G2 402

DATEL, NWT 200/800 + MII G2 8300 


\begin{tabular}{|c|c|c|c|c|c|}
\hline Star & & & & & \\
\hline HJD-2400000 & Error & I/II Method & Points & Observer & Technique \\
\hline 58005.53779 & 0.0004 & I $\quad \mathrm{CCD}+\mathrm{R}$ & $172 / 136$ & Hanžl D. & $\begin{array}{l}\text { DATEL, NWT } 200 / 800+\text { MII } \\
\text { G2 } 8300\end{array}$ \\
\hline
\end{tabular}

V0942 Cep

58182.28392

V0951 Cep

57995.33668

57995.33669

V0959 Cep

57966.37539

57974.38093

57987.41068

V0960 Cep

57966.48499

57974.50126

58052.32383

VW Cep

57922.46423

XX Cep

57690.43913

XY Cep

58027.36830

58052.33795

58052.33997
0.0007 I DSLR

0.0002 I CCD+Clear 201/77 Urbaník M.

$0.0002 \mathrm{I} \quad \mathrm{CCD}+\mathrm{R} \quad 88 / 23 \quad$ Hanžl D.

0.0003 II $\quad \mathrm{CCD}+$ Clear $\quad 316 / 74 \quad$ Urbaník M.

0.0005 I CCD+Clear 266/47 Urbaník M.

0.0005 I CCD + Clear $\quad 160 / 43 \quad$ Ehrenberger R.

0.0002 I CCD+Clear 313/267 Urbaník M.

0.0003 I CCD+Clear 266/214 Urbaník M.

0.0004 I CCD + Clear 107/75 Walter F., Hadík B.

$75 / 28$ Walter F.

0.0008 II DSLR

$0.0004 \mathrm{I} \quad \mathrm{CCD}+$ Clear $175 / 87$ Bragagnolo U

Sky-Watcher REF 100/900 QHY9

Tamron SP LNS 62.5/500 +

Canon 350 D

Sky-Watcher NWT 150/750 + MII G2-8300

Sky-Watcher NWT 150/750 + MII G2-8300

$\mathrm{CZ}$ Kometensucher REF 200/1370 + SBIG ST7

Sky-Watcher NWT 150/750 + MII G2-8300

Sky-Watcher NWT 150/750 + MII G2-8300

G2-8300

Tamron LNS 44/300 + Canon $600 \mathrm{D}$

CZ Mirotar LNS 180/1000 + Canon 350D dík B.

$\mathrm{CZ}$ Kometensucher 200/1370 + SBIG ST7 


\section{Star}

HJD-2400000

Error I/II Method

Points Observer

Technique

ASAS J022810-0659.4 Cet

57713.32385

0.0003

CCD + Clear 796/269 Lomoz F.

STN 254/1016+G2-8300

EE Cet

58026.54837

0.0001 II $\quad \mathrm{CCD}+\mathrm{R} \quad 110 / 69$ Mašek M.

NWT 150/600 + CCD MII G2-1600 + comacorector

SX CMa

57744.63232

0.001 I $\quad \mathrm{CCD}+\mathrm{R} \quad 84 / 56 \quad$ Mašek M.

FRAM, Nikkor LNS 106/300

+ G4-16000

AO CMi

57811.33038

0.0001 I CCD + Clear 98/65 Trnka J.

Skywatcher NWT 200/1000+

SBIG ST-9E + comacorre

AV CMi

58173.29730

0.0002 I CCD + Clear $\quad 89 / 29$ Mašek M.

NWT 150/600 + CCD MII

G2-1600 + comacorector

CX CMi

57798.53314

0.0007 II $\mathrm{CCD}+\mathrm{R}$

120/79 Smolka M.

NWT $250 / 1000+$ ATIK $314 \mathrm{~L}++\mathrm{ACC}$

EL CMi

57776.48794

0.0008 II $\quad$ CD + Clear $79 / 37 \quad$ Mašek M.

NWT 150/600 + CCD MII

G2-1600 + comacorector

ASAS J083128+1953.1 Cnc

\begin{tabular}{|c|c|c|c|c|c|c|c|}
\hline 57714.57414 & 0.0005 & I & $\mathrm{CCD}+\mathrm{V}$ & $135 / 80$ & Šmelcer L. & $\begin{array}{l}\text { Celestron SCT } \\
\text { MII G2-1600 }\end{array}$ & $355 / 2460$ \\
\hline 57715.44827 & 0.0006 & I & $\mathrm{CCD}+\mathrm{V}$ & $191 / 19$ & Šmelcer L. & $\begin{array}{l}\text { Celestron SCT } \\
\text { MII G2-1600 }\end{array}$ & $355 / 2460$ \\
\hline 57715.56158 & 0.0004 & II & $\mathrm{CCD}+\mathrm{V}$ & $191 / 150$ & Šmelcer L. & $\begin{array}{l}\text { Celestron SCT } \\
\text { MII G2-1600 }\end{array}$ & $355 / 2460$ \\
\hline 57752.36968 & 0.0007 & II & $\mathrm{CCD}+\mathrm{B}$ & $201 / 26$ & Šmelcer L. & $\begin{array}{l}\text { Celestron SCT } \\
\text { MII G2-4000 }\end{array}$ & $280 / 1765$ \\
\hline 57752.47296 & 0.0004 & $\mathrm{I}$ & $\mathrm{CCD}+\mathrm{B}$ & $201 / 132$ & Šmelcer L. & $\begin{array}{l}\text { Celestron SCT } \\
\text { MII G2-4000 }\end{array}$ & $280 / 1765$ \\
\hline 57783.29448 & 0.0013 & II & $\mathrm{CCD}+\mathrm{B}$ & $353 / 27$ & Šmelcer L. & $\begin{array}{l}\text { Celestron SCT } \\
\text { MII G2-4000 }\end{array}$ & $280 / 1765$ \\
\hline
\end{tabular}




\begin{tabular}{|c|c|c|c|c|c|c|c|c|}
\hline Star & & & & & & & & \\
\hline HJD-2400000 & Error & $\mathrm{I} / \mathrm{II}$ & Method & Points & Observer & Technique & & \\
\hline 57783.29785 & 0.0004 & II & $\mathrm{CCD}+\mathrm{V}$ & $374 / 49$ & Šmelcer L. & $\begin{array}{l}\text { Celestron SCT } \\
\text { MII G2-1600 }\end{array}$ & $355 / 2460$ & + \\
\hline 57783.39596 & 0.0004 & I & $\mathrm{CCD}+\mathrm{V}$ & $374 / 146$ & Šmelcer L. & $\begin{array}{l}\text { Celestron SCT } \\
\text { MII G2-1600 }\end{array}$ & $355 / 2460$ & + \\
\hline 57783.40023 & 0.0004 & $\mathrm{I}$ & $\mathrm{CCD}+\mathrm{B}$ & $353 / 136$ & Šmelcer L. & $\begin{array}{l}\text { Celestron SCT } \\
\text { MII G2-4000 }\end{array}$ & $280 / 1765$ & + \\
\hline 57783.51755 & 0.0006 & II & $\mathrm{CCD}+\mathrm{V}$ & $374 / 277$ & Šmelcer L. & $\begin{array}{l}\text { Celestron SCT } \\
\text { MII G2-1600 }\end{array}$ & $355 / 2460$ & + \\
\hline 57783.52278 & 0.0005 & II & $\mathrm{CCD}+\mathrm{B}$ & $353 / 269$ & Šmelcer L. & $\begin{array}{l}\text { Celestron SCT } \\
\text { MII G2- } 4000\end{array}$ & $280 / 1765$ & + \\
\hline 57799.29778 & 0.0003 & I & $\mathrm{CCD}+\mathrm{V}$ & $342 / 51$ & Šmelcer L. & $\begin{array}{l}\text { Celestron SCT } \\
\text { MII G2-1600 }\end{array}$ & $355 / 2460$ & + \\
\hline 57799.29896 & 0.0004 & $\mathrm{I}$ & $\mathrm{CCD}+\mathrm{B}$ & $364 / 71$ & Šmelcer L. & $\begin{array}{l}\text { Celestron SCT } \\
\text { MII G2- } 4000\end{array}$ & $280 / 1765$ & + \\
\hline 57799.41677 & 0.0005 & II & $\mathrm{CCD}+\mathrm{B}$ & $364 / 204$ & Šmelcer L. & $\begin{array}{l}\text { Celestron SCT } \\
\text { MII G2- } 4000\end{array}$ & $280 / 1765$ & + \\
\hline 57799.41770 & 0.0004 & II & $\mathrm{CCD}+\mathrm{V}$ & $342 / 181$ & Šmelcer L. & $\begin{array}{l}\text { Celestron SCT } \\
\text { MII G2-1600 }\end{array}$ & $355 / 2460$ & + \\
\hline 57799.51733 & 0.0003 & I & $\mathrm{CCD}+\mathrm{V}$ & $342 / 282$ & Šmelcer L. & $\begin{array}{l}\text { Celestron SCT } \\
\text { MII G2-1600 }\end{array}$ & $355 / 2460$ & + \\
\hline 57799.51746 & 0.0004 & $\mathrm{I}$ & $\mathrm{CCD}+\mathrm{B}$ & $364 / 306$ & Šmelcer L. & $\begin{array}{l}\text { Celestron SCT } \\
\text { MII G2- } 4000\end{array}$ & $280 / 1765$ & + \\
\hline 57800.28693 & 0.0004 & II & $\mathrm{CCD}+\mathrm{V}$ & $195 / 55$ & Šmelcer L. & $\begin{array}{l}\text { Celestron SCT } \\
\text { MII G2-1600 }\end{array}$ & $355 / 2460$ & + \\
\hline 57800.28954 & 0.0006 & II & $\mathrm{CCD}+\mathrm{B}$ & $412 / 56$ & Šmelcer L. & $\begin{array}{l}\text { Celestron SCT } \\
\text { MII G2-4000 }\end{array}$ & $280 / 1765$ & + \\
\hline 57800.38879 & 0.0004 & $\mathrm{I}$ & $\mathrm{CCD}+\mathrm{B}$ & $412 / 171$ & Šmelcer L. & $\begin{array}{l}\text { Celestron SCT } \\
\text { MII G2-4000 }\end{array}$ & $280 / 1765$ & + \\
\hline 57800.50763 & 0.0005 & II & $\mathrm{CCD}+\mathrm{B}$ & $412 / 296$ & Šmelcer L. & $\begin{array}{l}\text { Celestron SCT } \\
\text { MII G2-4000 }\end{array}$ & $280 / 1765$ & + \\
\hline 57800.60673 & 0.0013 & I & $\mathrm{CCD}+\mathrm{B}$ & $412 / 401$ & Šmelcer L. & $\begin{array}{l}\text { Celestron SCT } \\
\text { MII G2- } 4000\end{array}$ & $280 / 1765$ & + \\
\hline 57801.26321 & 0.0006 & $\mathrm{I}$ & $\mathrm{CCD}+\mathrm{B}$ & $94 / 26$ & Šmelcer L. & $\begin{array}{l}\text { Celestron SCT } \\
\text { MII G2-4000 }\end{array}$ & $280 / 1765$ & + \\
\hline 57825.43625 & 0.0008 & $\mathrm{I}$ & $\mathrm{CCD}+\mathrm{B}$ & $163 / 47$ & Šmelcer L. & $\begin{array}{l}\text { Celestron SCT } \\
\text { MII G2-4000 }\end{array}$ & $280 / 1765$ & + \\
\hline 57828.39039 & 0.0008 & II & $\mathrm{CCD}+\mathrm{B}$ & $267 / 116$ & Šmelcer L. & $\begin{array}{l}\text { Celestron SCT } \\
\text { MII G2-4000 }\end{array}$ & $280 / 1765$ & + \\
\hline 57828.48556 & 0.0005 & $\mathrm{I}$ & $\mathrm{CCD}+\mathrm{B}$ & $267 / 207$ & Šmelcer L. & $\begin{array}{l}\text { Celestron SCT } \\
\text { MII G2-4000 }\end{array}$ & $280 / 1765$ & + \\
\hline 57839.37860 & 0.0007 & I & $\mathrm{CCD}+\mathrm{B}$ & $234 / 75$ & Šmelcer L. & $\begin{array}{l}\text { Celestron SCT } \\
\text { MII G2-4000 }\end{array}$ & $280 / 1765$ & + \\
\hline 57839.48966 & 0.0007 & II & $\mathrm{CCD}+\mathrm{B}$ & $234 / 210$ & Šmelcer L. & $\begin{array}{l}\text { Celestron SCT } \\
\text { MII G2-4000 }\end{array}$ & $280 / 1765$ & + \\
\hline 57840.36561 & 0.0005 & II & $\mathrm{CCD}+\mathrm{B}$ & $204 / 82$ & Šmelcer L. & $\begin{array}{l}\text { Celestron SCT } \\
\text { MII G2-4000 }\end{array}$ & $280 / 1765$ & + \\
\hline 57853.43504 & 0.0007 & II & $\mathrm{CCD}+\mathrm{B}$ & $128 / 103$ & Šmelcer L. & $\begin{array}{l}\text { Celestron SCT } \\
\text { MII G2-4000 }\end{array}$ & $280 / 1765$ & + \\
\hline
\end{tabular}




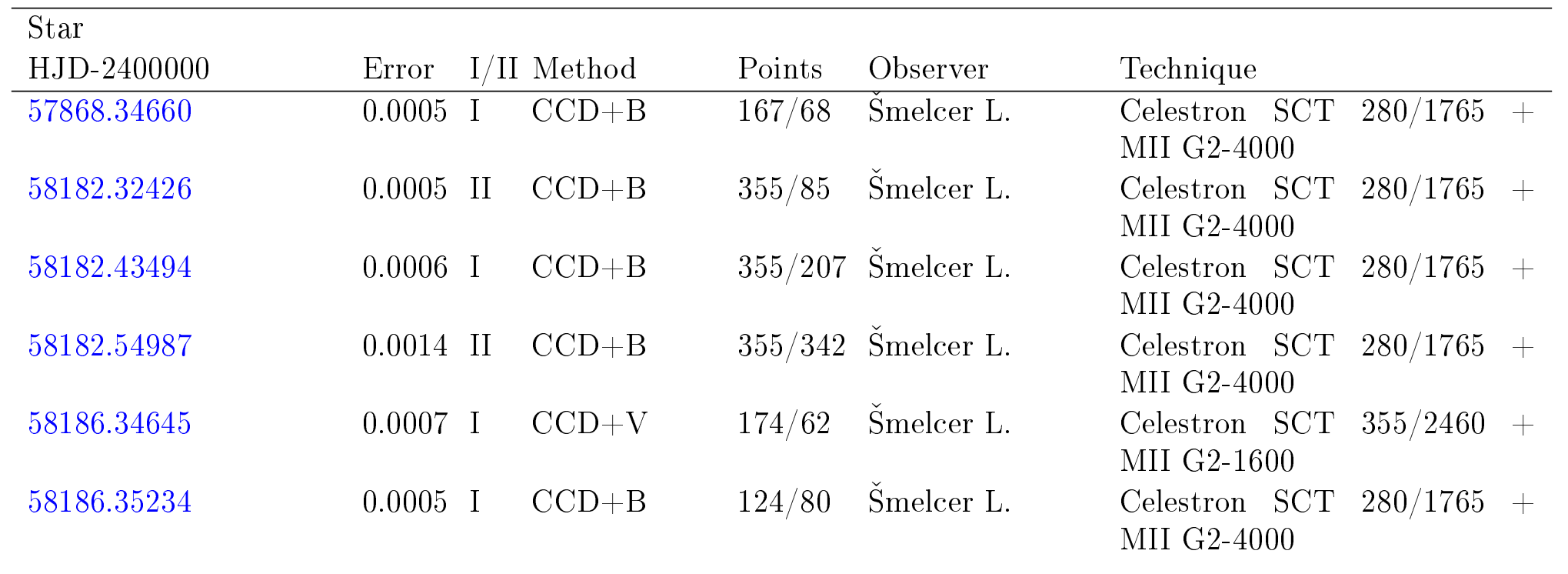

CSS J083735.4+093914 Cnc

58113.65063

$\mathrm{EH} \mathrm{Cnc}$

57762.47609

FF Cnc

58174.41910

HN Cnc

57839.30974

IR Cnc

57825.38741

IT Cnc

57435.38741

57783.41305

LL Cnc

57839.45625

LM Cnc

58179.48060
0.0008 I CCD + Clear 214/157 Lomoz F.

$0.0001 \mathrm{I} \quad \mathrm{CCD}+\mathrm{R} \quad 192 / 40 \quad$ Šmelcer L.

0.0001 I CCD + Clear $70 / 28$ Mašek M.

0.0002 I $\quad C C D+$ Clear $129 / 58$ Lehky M.

0.0003 I CCD + Clear $\quad 120 / 58 \quad$ Smolka M.

0.0006 II CCD + Clear 242/154 Jacobsen J.

0.0004 II DSLR

148/68 Nosál' P.

0.0006 I $\quad C C D+$ Clear $\quad 90 / 68$ Mašek M.
STN 254/1016+CCD-G2-8300

Celestron SCT 280/1765 + MII G2-4000

NWT $150 / 600+$ CCD MII G2-1600 + comacorector

HK25， NWT 250/1000 + SBIG ST-7 + C

NWT $250 / 1000+$ ATIK $314 \mathrm{~L}++\mathrm{ACC}$

EQ6, $80 \mathrm{~mm}$ refractor, SXVRH16

REF 102/500 + Canon 20D 


\begin{tabular}{|c|c|c|c|c|c|}
\hline \\
\hline HJD-2400000 & Error & I/II Method & Points & Observer & Technique \\
\hline 57800.37998 & 0.0002 & I $\quad$ CCD + Clear & $78 / 24$ & Walter F. & $\begin{array}{l}\text { MARK SCT 406/4060, SBIG } \\
\text { ST10XME }\end{array}$ \\
\hline 57800.38020 & 0.0003 & $\mathrm{CCD}+\mathrm{R}$ & $77 / 21$ & Walter F. & $\begin{array}{l}\text { MARK SCT 406/4060, SBIG } \\
\text { ST10XME }\end{array}$ \\
\hline 57800.38068 & 0.0003 & $\mathrm{CCD}+\mathrm{I}$ & $72 / 22$ & Walter F. & $\begin{array}{l}\text { MARK SCT 406/4060, SBIG } \\
\text { ST10XME }\end{array}$ \\
\hline 57827.37043 & 0.0005 & DSLR & $140 / 77$ & Nosál P. & $\begin{array}{l}\text { NWT } 150 / 600+\text { Canon 20D + } \\
\text { MPCC mk.III }\end{array}$ \\
\hline 57845.36066 & 0.0001 & $\mathrm{CCD}+$ Clear & $130 / 81$ & Trnka J. & $\begin{array}{l}\text { Skywatcher NWT } 200 / 1000+ \\
\text { SBIG ST-9E + comacorre }\end{array}$ \\
\hline
\end{tabular}

YY Cnc

57842.43003
0.0004 I CCD +Clear 407/262 Bragagnolo U.
Sky-Watcher REF 100/900 + QHY9

BPS BS 16033-028

Com

57892.38285

0.0008 I DSLR $\quad 58 / 33 \quad$ Nosál' P.

NWT $150 / 600+$ Canon 20D + MPCC mk.III

CC Com

57751.53964

0.0001 II $\mathrm{CCD}+$ Clear

531/115 Červinka L.

$\mathrm{R} 102 / 500+$ Atik 314L +

57751.65003

0.0001 I $\mathrm{CCD}+$ Clear

531/341 Červinka L.

R102/500 + Atik 314L +

57845.44113

0.0001 I CCD + Clear 75/33 Mašek M.

NWT $150 / 600+$ CCD MII G2-1600 + comacorector

EK Com

57846.38745

0.0001 I $\quad$ CCD + R $\quad 239 / 119$ Šmelcer L.

Celestron SCT 355/2460

MII G2-1600

LL Com

57843.34254

$0.0005 \mathrm{I} \quad \mathrm{CCD}+\mathrm{R} \quad 43 / 16 \quad$ Lehky $\mathrm{M}$.

HK25, NWT 250/1000

SBIG ST-7 + Rc

LO Com

58202.55899

0.0004 I CCD + Clear 155/127 Urbaník M.

Sky-Watcher REF $80 / 520$

MII G2-8300

LP Com

58202.55081

0.0006 II CCD+Clear 154/115 Urbaník M.

Sky-Watcher REF $80 / 520$

MII G2-8300 
Star

HJD-2400000

LR Com

57840.46482

MM Com

57853.37245

MR Com

57754.61071

NN Com

57800.51977

57800.52084

58149.64313

NV Com

57869.45739

RW Com

57752.51813

57827.40337

58202.52775

RZ Com

57776.50381

57776.67356

TZ CrA

57951.58895

V0634 CrA

58063.51059
0.0003 II $\quad$ CCD + Clear $56 / 35 \quad$ Mašek M.

$0.0004 \mathrm{I} \quad \mathrm{CCD}+\mathrm{R} \quad 119 / 65 \quad$ Lehky M.

0.0002 I CCD + Clear 465/174 Magris M.

Achromatic refractor 120/600,

CCD Orion Starshoo

HK25, NWT 250/1000 + SBIG ST-7 + Rc

NWT $150 / 600+$ CCD MII G2-1600 + comacorector

$\begin{array}{lllll}0.0003 & \text { I } & \text { CCD }+\mathrm{I} & 64 / 27 & \text { Walter F. } \\ 0.0003 \text { I } & \text { CCD }+ \text { Clear } & 57 / 27 & \text { Walter F. } \\ 0.0011 \text { I } & \text { CCD }+ \text { Clear } & 165 / 109 & \text { Nosál' P. }\end{array}$

0.0014 II DSLR 111/76 Nosál' P.

NWT $150 / 600+$ Canon 20D + MPCC mk.III

0.0002 II $\quad$ CCD + Clear $284 / 121$ Červinka L. $\quad$ R102/500 + Atik 314L +

0.0003 I DSLR 61/26 Nosál P. REF 102/500 + Canon 20D

0.0001 II CCD + Clear 159/90 Urbaník M. Sky-Watcher REF 80/520 +

MII G2-8300

0.0001 II $\quad$ CCD + Clear $856 / 246$ Červinka L. $\quad$ R102/500 + Atik 314L +

0.0001 I $\quad$ CCD + Clear $856 / 785$ Červinka L. R102/500 + Atik 314L +

$0 \quad \mathrm{I} \quad \mathrm{CCD}+\mathrm{R} \quad 166 / 107$ Mašek M. FRAM, Meade SCT 300/3000

+ MII G2-1600 


\begin{tabular}{|c|c|c|c|c|c|c|}
\hline $\begin{array}{l}\text { Star } \\
\text { HJD-2400000 }\end{array}$ & Error & $\mathrm{I} / \mathrm{I}$ & Method & Points & Observer & Technique \\
\hline AW CrB & & & & & & \\
\hline $\begin{array}{l}57883.39761 \\
\text { CL CrB }\end{array}$ & 0.0004 & I & $\mathrm{CCD}+$ Clear & $196 / 142$ & Červinka L. & NWT 200/1000 + Atik 314L + \\
\hline 57473.37852 & 0.0006 & I & $\mathrm{CCD}+$ Clear & $181 / 55$ & Jacobsen J. & $\begin{array}{l}\text { EQ6, } 80 \mathrm{~mm} \text { refractor, SXVR- } \\
\text { H16 }\end{array}$ \\
\hline 57892.45600 & 0.0002 & II & $\mathrm{CCD}+$ Clear & $122 / 70$ & Ehrenberger R. & $\begin{array}{l}\text { Quadruplet LNS } 65 / 420+\mathrm{MII} \\
\text { G2-8300 }\end{array}$ \\
\hline 57977.41472 & 0.0004 & I & $\mathrm{CCD}+$ Clear & $159 / 85$ & Bragagnolo U. & $\begin{array}{l}\text { Sky-Watcher REF } 100 / 900+ \\
\text { QHY9 }\end{array}$ \\
\hline
\end{tabular}

CSS J160438.0+281501 CrB

$57876.44963 \quad 0.0003$ I $\quad$ CCD + Clear $\quad 711 / 260$ Lomoz F.

NSVS $07826147 \mathrm{CrB}$ 57892.40378

$0 \quad$ I $\quad$ CCD + Clear $\quad 126 / 34 \quad$ Šmelcer L.

\section{RW CrB}

57799.52127

TW CrB

57853.57235

57853.57242

YY CrB

57823.54658

57943.66811
0.0014 II $\quad$ CCD + Clear $803 / 308$ Červinka L.

0.0001 I $\quad \mathrm{CCD}+\mathrm{R} \quad 166 / 118$ Šmelcer L.

0.0001 I $\quad$ CCD $+\mathrm{I} \quad 167 / 115$ Šmelcer L.
0.0004 I CCD + Clear 231/107 Červinka L.

0.0002 I Photometer + V62/33 Persha G.
SNT 254/1016+G2-8300

Celestron SCT 280/1765 + MII G2-4000

R102/500 + Atik 314L +

Celestron SCT 280/1765 + MII G2-4000

Celestron SCT 280/1765 + MII G2-4000

R102/500 + Atik 314L +

Meade RCT 250/2500 + Optec SSP-5a

\section{BI CVn}

57846.36316

\section{BO CVn}

57843.40342

57910.67398

0.0002 I CCD +Clear 181/100 Vrašták M.

0.0002 I CCD +Clear 489/89 Vrašták M

0.0002 I Photometer + V50/26 Persha G.
RF 80/400+G1-0301

Meade RCT 250/2500 + Optec SSP-5a

CSS J122541.6+352314 CVn

57845.40106

0.0003 I DSLR $\quad 146 / 78$ Nosál P.

NWT 150/600 + Canon 20D + MPCC mk.III 


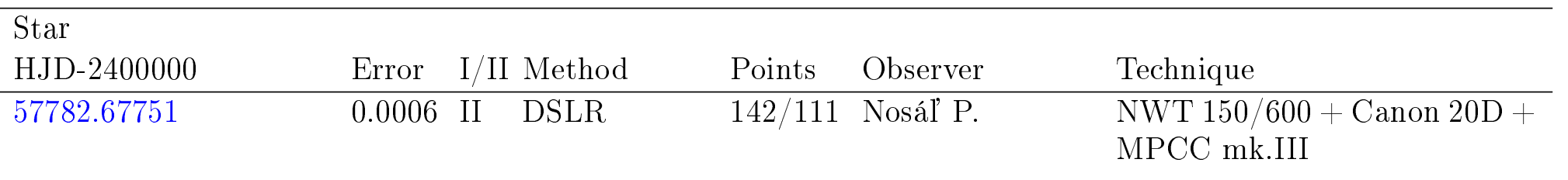

DH CVn

57845.44273

DL CVn

57760.63223

58112.69425

DR CVn

57844.32495

57844.32641

57844.32649

57844.32748

DU CVn

57760.63219

DY CVn

57799.56227

EF CVn

57841.57366

EG CVn

57901.52582

57902.40329

EI CVn

57902.41844

0.0006 I DSLR 146/108 Nosál P.

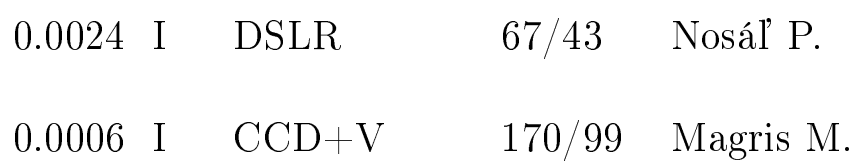

0.0006 II $\mathrm{CCD}+\mathrm{B} \quad 26 / 12 \quad$ Vrašták M.

0.0003 II $\mathrm{CCD}+\mathrm{V} \quad 26 / 13$ Vrašták M.

0.0004 II $\quad \mathrm{CCD}+\mathrm{R} \quad 26 / 14 \quad$ Vrašták M

0.0003 II $\quad \mathrm{CCD}+\mathrm{I} \quad 26 / 14 \quad$ Vrašták M

0.0016 I DSLR $\quad 58 / 36 \quad$ Nosál P.

0.0003 I $\quad \mathrm{CCD}+$ Clear $\quad 59 / 45$

Walter F., Hladík B.

$\mathrm{CZ}$ Kometensucher REF 200/1370 + SBIG ST7

0.0004 II $\quad \mathrm{CCD}+\mathrm{R} \quad 114 / 64 \quad$ Lehky M.

HK25, NWT 250/1000 + SBIG ST-7 + Rc

NWT $150 / 600+$ Canon 20D + MPCC mk.III

NWT 280/1500+MII G2-1600, $80 / 400+$ G1-0301

NWT 280/1500+MII G2-1600, 80/400+G1-0301

NWT 280/1500+MII G2-1600, $80 / 400+$ G1-0301

NWT 280/1500+MII G2-1600, $80 / 400+$ G1-0301

HK25, NWT 250/1000 SBIG ST-7 + BVRcIc HK25, NWT 250/1000 + SBIG ST-7 + BVRcIc

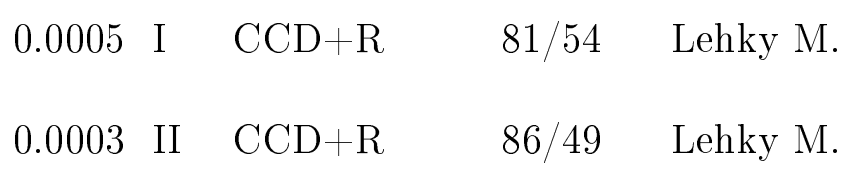

$0.0001 \mathrm{I} \quad \mathrm{CCD}+$ Clear $\quad 174 / 54 \quad$ Bragagnolo U.

Sky-Watcher REF 100/900 + QHY9 


\begin{tabular}{|c|c|c|c|c|c|}
\hline Star & & & & & \\
\hline HJD-2400000 & Error & I/II Method & Points & Observer & Technique \\
\hline 57853.48067 & 0.0008 & II $\quad$ DSLR & $195 / 132$ & Nosál' P. & $\begin{array}{l}\text { NWT } 150 / 600+\text { Canon 20D }+ \\
\text { MPCC mk.III }\end{array}$ \\
\hline
\end{tabular}

EV CVn

57757.54436

57844.38952

EX CVn

57782.57450

FU CVn

57847.36343

FY CVn

57802.60176

0.0009 II DSLR

FZ CVn

57802.56086

0.0006 I DSLR

129/31 Nosál P.

GM CVn

58199.38432

0.0002 I $\quad$ CCD + Clear

130/59 Nosál' P.

NWT 150/600 + Canon 20D + MPCC mk.III

ROTSE1 J122809.53+353339.1 CVn 57845.38994

0.0004 I DSLR

146/70 Nosál' P.

TSVSC1 TN-N033313030-4-67-2 Cyg

57645.30006

0.0001 I CCD + Clear 269/66 Lomoz F.

1SWASP J212212.08+353121.5 Cyg

57964.44888

0.0002 I CCD + Clear 174/107 Urbaník M.

2MASS J19375316+3931511 Cyg

57929.43180

I CCD +Clear 238/124 Urbaník M.

Achromatic refractor 120/600, CCD Orion Starshoo

NWT 150/600 + Canon 20D + MPCC mk.III

NWT $150 / 600+$ Canon 20D + MPCC mk.III

Sky-Watcher NWT 150/750 + MII G2-8300

NWT 150/600 + Canon 20D + MPCC mk.III

NWT 150/600+MII G2-8300

NWT 150/600 + Canon 20D + MPCC mk.III

STN 254/1016+G2-8300

Sky-Watcher NWT 150/750 + MII G2-8300 


\begin{tabular}{lllllll}
\hline Star & & & & & \\
HJD-2400000 & Error & I/II Method & Points & Observer & Technique & \\
\hline 57884.50020 & 0.0007 & I & CCD + R & $72 / 41$ & Mazanec J. & ORION, NWT $500 / 1500+$ \\
& & & & & & MII G2 402
\end{tabular}

ASAS J193049+4635.3 Cyg

57895.51991

0.001 I $\quad$ CCD + Clear $187 / 151$ Červinka L.

R102/500 + Atik 320E mono, $0.5 \mathrm{x} \mathrm{FC}$

ASAS J200524+4411.3 Cyg

57895.44203

0.0003 I CCD+Clear 143/61 Mašek M., Tylšar M.

NWT 250/1000 + CCD MII G2-8300

BR Cyg

57952.65757

CzeV1056 Cyg

57590.37965

57590.52576

57608.42091

57608.56737

57609.44782

57614.43529

57614.58087

57966.46714

57973.36127

57973.50765

57980.40184

57980.54844

CzeV261 Cyg

57915.44834

57916.45445
0.0002 I Photometer + V47/25 Persha G.

0.0002 II CCD +Clear 441/77 Búlek F.

0.0003 I CCD +Clear 441/325 Bílek F.

0.0001 I CCD +Clear 517/190 Bílek F.

0.0001 II CCD +Clear 517/440 Bílek F.

0.0002 II CCD+Clear 343/229 Bílek F.

0.0002 II CCD + Clear 561/235 Bílek F.

0.0002 I CCD +Clear 561/495 Bílek F.

0.0002 II CCD +Clear 452/229 Bílek F.

0.0003 I $\quad$ CCD + Clear $\quad 475 / 69 \quad$ Bílek F.

0.0003 II CCD+Clear 475/304 Bílek F.

0.0001 I $\quad$ CCD + Clear 501/153 Bílek F.

0.0002 II CCD + Clear 501/388 Búlek F.
Meade RCT 250/2500 + Optec SSP-5a

NWT 200/860 + CCD Atik $314 \mathrm{~L}+$

NWT 200/860 + CCD Atik $314 \mathrm{~L}+$

NWT 200/860 + CCD Atik $314 \mathrm{~L}+$

NWT 200/860 + CCD Atik $314 \mathrm{~L}+$

NWT 200/860 + CCD Atik $314 \mathrm{~L}+$

NWT 200/860 + CCD Atik $314 \mathrm{~L}+$

NWT 200/860 + CCD Atik $314 \mathrm{~L}+$

NWT 200/860 + CCD Atik $314 \mathrm{~L}+$

NWT 200/860 + CCD Atik $314 \mathrm{~L}+$

NWT 200/860 + CCD Atik $314 \mathrm{~L}+$

NWT 200/860 + CCD Atik $314 \mathrm{~L}+$

NWT 200/860 + CCD Atik $314 \mathrm{~L}+$

MNT $\quad 190 / 1000$ ATIK414EXM

Sky-Watcher NWT 150/750 + MII G2-8300 


\begin{tabular}{lcccll}
\hline $\begin{array}{l}\text { Star } \\
\text { HJD-2400000 }\end{array}$ & Error & I/II Method & Points & Observer & Technique \\
\hline $\begin{array}{l}\text { Czev750 Cyg } \\
57261.52289\end{array}$ & 0.001 & I & DSLR & $217 / 124$ Walter F. & $\begin{array}{l}\text { Zeiss MAK } 350 / 3300+\text { Canon } \\
350 \text { D }\end{array}$ \\
57265.55408 & 0.0006 & I & DSLR & $230 / 115$ Walter F. & $\begin{array}{l}\text { Zeiss MAK 350/3300 + Canon } \\
350 \text { D }\end{array}$
\end{tabular}

CzeV929 Cyg

58009.42721

DK Cyg

57214.45528

57984.51664

57984.51732

GSC 03543-00836 Cyg

57956.44976

57963.47909

KIC 11341950 Cyg

57626.46819

KIC 9472174 Cyg

57590.43751

57608.42030

57608.54903

57609.42693

57614.45930

57966.47512

57973.39185

57973.51983

57980.43568

57980.56334
0.0002 I CCD + Clear $187 / 41 \quad$ Walter F.

0.0002 II CCD +Clear 286/199 Ehrenberger R.

0.0005 II DSLR

0.0007 II DSLR

117/87 Walter F.

117/87 Walter F.

0.0003 I $\quad \mathrm{CCD}+\mathrm{R}$

0.0003 I $\quad \mathrm{CCD}+\mathrm{R}$

277/130 Vala J., Jíra S.

$220 / 94$ Vala J.

0.0003 I $\quad$ CCD + Clear $\quad 91 / 26$

Mašek M., Mrňák P.

MARK SCT 406/4060, SBIG ST10XME

Quadruplet LNS 65/420+MII G2-8300

Tamron SP LNS 62.5/500 Canon 350 D

Tamron SP LNS 62.5/500 + Canon 350 D

NWT 150/750 + MII G2-1600

NWT 150/750 + MII G2-1600

VSET, NWT 150/750 + CCD MII G2-1600

NWT 200/860 + CCD Atik $314 \mathrm{~L}+$

NWT 200/860 + CCD Atik $314 \mathrm{~L}+$

NWT 200/860 + CCD Atik $314 \mathrm{~L}+$

NWT 200/860 + CCD Atik $314 \mathrm{~L}+$

NWT 200/860 + CCD Atik $314 \mathrm{~L}+$

NWT 200/860 + CCD Atik $314 \mathrm{~L}+$

NWT 200/860 + CCD Atik $314 \mathrm{~L}+$

NWT 200/860 + CCD Atik $314 \mathrm{~L}+$

NWT 200/860 + CCD Atik $314 \mathrm{~L}+$

NWT 200/860 + CCD Atik $314 \mathrm{~L}+$ 


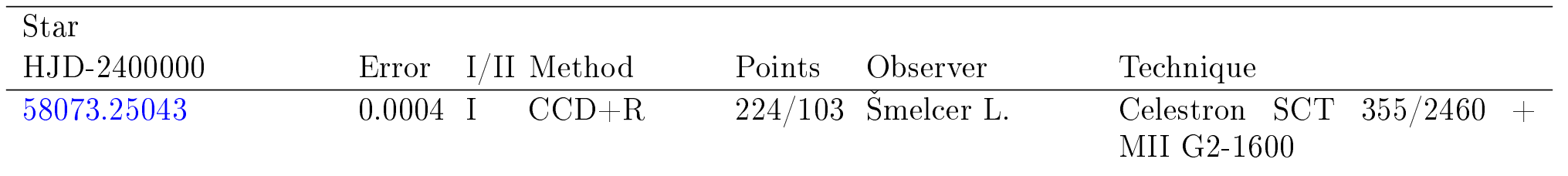

KIC 9533706 Cyg 57590.44705

57608.48819

57609.48307

57614.45531

KIC 9594768 Cyg 57608.50018

57609.39239

57614.50696

KIC 9649493 Cyg 57895.49927

57901.43549

KIC 9772642 Cyg 57901.43942

KIC 9832227 Cyg 57896.46065

57901.50147

57902.41688

57926.46488

57954.39859

57956.46726 57963.55381
0.0005 I CCD + Clear 311/178 Bílek F.

0.0003 II $\mathrm{CCD}+$ Clear 530/310 Bílek F.

0.0003 II CCD+Clear 353/292 Bílek F.

0.0003 II CCD + Clear 553/261 Bílek F.

0.0008 II CCD + Clear 520/321 Bílek F.

0.001 II CCD +Clear 343/137 Bílek F.

0.0008 I $\quad$ CCD +Clear 542/347 Bílek F.

0.0014 I $\quad$ CCD + Clear $186 / 129$ Červinka L.

0.001 I $\quad$ CCD + Clear $442 / 165$ Červinka L.

0.0011 I $\quad$ CCD + Clear $\quad 415 / 163$ Červinka L.

0.001 I CCD + Clear 210/109 Červinka L .

0.0007 I CCD + Clear $440 / 301$ Červinka L.

0.0009 I CCD + Clear 365/115 Červinka L.

0.0003 I $\quad$ CCD + Clear 170/95 Bílek F .

0.0007 I $\quad \mathrm{CCD}+\mathrm{R}$

0.0006 II $\mathrm{CCD}+\mathrm{R}$

0.0017 I $\mathrm{CCD}+\mathrm{R}$
144/71 Vala J., S. Jíra 285/152 Vala J., S. Jíra 232/192 CCD group in Upice observatory
NWT 200/860 + CCD Atik $314 \mathrm{~L}+$

NWT 200/860 + CCD Atik $314 \mathrm{~L}+$

NWT 200/860 + CCD Atik $314 \mathrm{~L}+$

NWT 200/860 + CCD Atik $314 \mathrm{~L}+$

NWT 200/860 + CCD Atik $314 \mathrm{~L}+$

NWT 200/860 + CCD Atik $314 \mathrm{~L}+$

NWT 200/860 + CCD Atik $314 \mathrm{~L}+$

R102/500 + Atik 320E mono, $0.5 \mathrm{x}$ FC

R102/500 + Atik 320E mono, $0.5 \mathrm{x}$ FC

R102/500 + Atik 320E mono, $0.5 \mathrm{x}$ FC

R102/500 + Atik 320E mono, $0.5 \mathrm{x}$ FC

R102/500 + Atik 320E mono, $0.5 \mathrm{x} F \mathrm{~F}$

R102/500 + Atik 320E mono, $0.5 \mathrm{x}$ FC

NWT 200/860 + CCD Atik $314 \mathrm{~L}+$

NWT 150/750 + MII G2-1600

NWT 150/750 + MII G2-1600

NWT 150/750 + MII G2-1600 


\begin{tabular}{|c|c|c|c|c|c|}
\hline Star & & & & & \\
\hline HJD-2400000 & Error & I/II Method & Points & Observer & Technique \\
\hline 57929.45551 & 0.0007 & I $\quad$ CCD + Clear & $236 / 158$ & Urbaník M. & $\begin{array}{l}\text { Sky-Watcher NWT 150/750 + } \\
\text { MII G2-8300 }\end{array}$ \\
\hline
\end{tabular}

KID 08719897 Cyg 57895.51049

\section{LN Cyg}

58041.33177

58041.33206

58041.33281

58041.33417

NSVS 3071474 Cyg

57900.47666

57900.47951

NSVS 3245311 Cyg 58047.31462

NSVS 5748348 Cyg 57954.40137

57971.37829

57973.44333

58101.22972

NSVS 5751296 Cyg 57954.40735

0.0002 I CCD+Clear 259/100 Urbaník M.

OO Cyg

57996.31088

57996.44250
0.0005 I CCD+Clear 138/111 Mašek M., Tylšar M.

NWT 250/1000 + CCD MII G2-8300

$0.0001 \mathrm{I} \quad \mathrm{CCD}+\mathrm{B} \quad 101 / 67 \quad$ Mazanec J.

0.0001 I $\quad \mathrm{CCD}+\mathrm{R} \quad 97 / 62 \quad$ Mazanec J.

$0.0002 \mathrm{I} \quad \mathrm{CCD}+\mathrm{V} \quad 82 / 53 \quad$ Mazanec J.

$0.0002 \quad \mathrm{I} \quad \mathrm{CCD}+\mathrm{I}$

85/58 Mazanec J.

$0.0003 \mathrm{I} \quad \mathrm{CCD}+\mathrm{R} \quad 360 / 188$ Lomoz F.

0.0001 I CCD + Clear 316/215 Lomoz F.

0.0004 I CCD+Clear 276/207 Urbaník M.

0.0002 I CCD+Clear 257/88 Urbaník M.

0.0002 I CCD+Clear 243/65 Urbaník M.

0.0009 I $\quad C C D+$ Clear 96/59 Urbaník M.

0.0004 I CCD+Clear 204/87 Urbaník M.
ORION, NWT 500/1500 MII G2 402

ORION, NWT 500/1500 + MII G2 402

ORION, NWT 500/1500 + MII G2 402

ORION, NWT 500/1500 + MII G2 402

Newton 300/1200+ST2000XM STN 254/1016+G2-8300

Sky-Watcher NWT 150/750 + MII G2-8300

Sky-Watcher NWT 150/750 + MII G2-8300

Sky-Watcher NWT 150/750 + MII G2-8300

Sky-Watcher NWT 150/750 + MII G2-8300

Sky-Watcher NWT 150/750 + MII G2-8300

Sky-Watcher NWT 150/750 + MII G2-8300

Sky-Watcher NWT 150/750 + MII G2-8300

Sky-Watcher NWT 150/750 + MII G2-8300 


\begin{tabular}{|c|c|c|c|c|c|}
\hline Star & & & & & \\
\hline H.JD-2400000 & Error & $\mathrm{I} / \mathrm{II}$ & Method & Points & Observer \\
\hline 57996.45325 & 0.0002 & $\mathrm{I}$ & CCD + Clear & $353 / 203$ & Urbaník M. \\
\hline PV Cyg & & & & & \\
\hline 57924.41168 & 0.0002 & I & CCD + Clear & $146 / 57$ & Urbaník M. \\
\hline QV Cyg & & & & & \\
\hline 57924.42933 & 0.0002 & I & CCD + Clear & $184 / 95$ & Urbaník M. \\
\hline SvkV132 Cyg & & & & & \\
\hline 57845.44515 & 0.0012 & II & CCD + Clear & $362 / 35$ & Urbaník M. \\
\hline 57845.59210 & 0.0004 & I & CCD + Clear & $362 / 274$ & Urbaník M. \\
\hline 57893.39572 & 0.0004 & I & CCD + Clear & $202 / 82$ & Urbaník M. \\
\hline 57913.43639 & 0.0004 & II & CCD + Clear & $218 / 118$ & Urbaník M. \\
\hline
\end{tabular}

V0348 Cyg 57915.39003

57915.39022

57915.39029

57919.36990

57919.37015

57919.37023

57919.51122

57919.51129

57919.51136

57931.44883

57931.44935

57931.44940
$0.0002 \mathrm{I} \quad \mathrm{CCD}+\mathrm{V} \quad 25 / 10 \quad$ Mazanec J.

$0.0002 \mathrm{I} \quad \mathrm{CCD}+\mathrm{R} \quad 27 / 12 \quad$ Mazanec J.

$0.0001 \mathrm{I} \quad \mathrm{CCD}+\mathrm{I} \quad 28 / 9 \quad$ Mazanec J.

$0.0003 \mathrm{I} \quad \mathrm{CCD}+\mathrm{V} \quad 94 / 14 \quad$ Mazanec J.

$0.0002 \mathrm{I} \quad \mathrm{CCD}+\mathrm{I} \quad 94 / 12 \quad$ Mazanec J.

0.0001 II $\quad \mathrm{CCD}+\mathrm{I} \quad 94 / 77 \quad$ Mazanec J.

0.0001 II $\mathrm{CCD}+\mathrm{R} \quad 95 / 78 \quad$ Mazanec J.

0.0001 II $\mathrm{CCD}+\mathrm{V} \quad 94 / 78 \quad$ Mazanec J.

0.0001 II $\mathrm{CCD}+\mathrm{V} \quad 96 / 48 \quad$ Mazanec J.

0.0001 II $\mathrm{CCD}+\mathrm{R} \quad 96 / 48 \quad$ Mazanec J.

0.0002 II $\mathrm{CCD}+\mathrm{I} \quad 97 / 48 \quad$ Mazanec J.
$0.0001 \mathrm{I} \quad \mathrm{CCD}+\mathrm{R} \quad 95 / 14 \quad$ Mazanec J.
Technique

Sky-Watcher NWT 150/750 + MII G2-8300

Sky-Watcher NWT 150/750 + MII G2-8300

Sky-Watcher NWT 150/750+ MII G2-8300

Sky-Watcher NWT 150/750 + MII G2-8300

Sky-Watcher NWT 150/750 + MII G2-8300

Sky-Watcher NWT 150/750 + MII G2-8300

Sky-Watcher NWT 150/750 MII G2-8300

ORION, NWT 500/1500 + MII G2 402

ORION, NWT 500/1500+ MII G2 402

ORION, NWT 500/1500

MII G2 402

ORION, NWT 500/1500+

MII G2 402

ORION, NWT 500/1500 + MII G2 402

ORION, NWT 500/1500 + MII G2 402

ORION, NWT 500/1500 + MII G2 402

ORION, NWT 500/1500 + MII G2 402

ORION, NWT 500/1500 MII G2 402

ORION, NWT 500/1500 + MII G2 402

ORION, NWT 500/1500 + MII G2 402

ORION, NWT 500/1500 + MII G2 402 


\begin{tabular}{|c|c|c|c|c|c|}
\hline Star & & & & & \\
\hline HJD-2400000 & Error & I/II Method & Points & Observer & Technique \\
\hline 57991.43833 & 0.0004 & I $\quad$ DSLR & $120 / 63$ & Walter F. & $\begin{array}{l}\text { Tamron SP LNS } 62.5 / 500+ \\
\text { Canon } 350 \text { D }\end{array}$ \\
\hline 57991.43851 & 0.0003 & DSLR & $128 / 62$ & Walter F. & $\begin{array}{l}\text { Tamron SP LNS } 62.5 / 500+ \\
\text { Canon } 350 \text { D }\end{array}$ \\
\hline
\end{tabular}

V0490 Cyg 57994.35007

57995.49043

58034.25844

V0496 Cyg 57996.39598

V0504 Cyg 58076.25455

V0505 Cyg 58091.27861

V0508 Cyg 57973.53970

57973.54000

57973.54009

57989.52302

57989.52319

57989.52394

V0700 Cyg 58019.35516

V0788 Cyg 57626.76649

\begin{tabular}{|c|c|c|c|c|}
\hline $0.0002 \mathrm{I}$ & $\mathrm{CCD}+$ Clear & $294 / 58$ & Urbaník M. & $\begin{array}{l}\text { Sky-Watcher NWT 150/750 } \\
\text { MII G2-8300 }\end{array}$ \\
\hline $0.0002 \mathrm{I}$ & $\mathrm{CCD}+$ Clear & $163 / 71$ & Urbaník M. & $\begin{array}{l}\text { Sky-Watcher NWT 150/750 } \\
\text { MII G2-8300 }\end{array}$ \\
\hline $0.0003 \mathrm{I}$ & $\mathrm{CCD}+$ Clear & $70 / 39$ & Urbaník M. & $\begin{array}{l}\text { Sky-Watcher NWT 150/750 + } \\
\text { MII G2-8300 }\end{array}$ \\
\hline
\end{tabular}

0.0001 II $\mathrm{CCD}+$ Clear $146 / 72$ Ehrenberger R. NWT 150/600+MII G2-8300

0.0004 I CCD +Clear 152/77 Bragagnolo U. Sky-Watcher REF 100/900 QHY9

0.0007 II CCD+Clear 276/90 Bragagnolo U. Sky-Watcher REF 100/900 QHY9

JST, NWT 400/2000 + MII G2-1600 + BVRcIc

JST, NWT 400/2000 + MII

G2-1600 + BVRcIc

JST, NWT 400/2000 + MII G2-1600 + BVRcIc

JST, NWT 400/2000 + MII

G2-1600 + BVRcIc

JST, NWT 400/2000 + MII G2-1600 + BVRcIc

JST, NWT 400/2000 + MII

G2-1600 + BVRcIc

$78 / 45$ Lehky M.
0.0001 I CCD +Clear 166/62 Bragagnolo U. Sky-Watcher REF 100/900 QHY9 


\begin{tabular}{|c|c|c|c|c|c|}
\hline Star & & & & & \\
\hline HJD-2400000 & Error & I/II Method & Points & Observer & Technique \\
\hline 57901.50350 & 0.0004 & I $\quad \mathrm{CCD}+$ Clear & $273 / 208$ & Červinka L. & $\begin{array}{l}\text { R102/500 + Atik 320E mono, } \\
0.5 x \text { FC }\end{array}$ \\
\hline
\end{tabular}

V0828 Cyg

58047.31140

V0836 Cyg

57918.48996

58026.62972

V0884 Cyg

57916.43282

V0891 Cyg

57906.42305

V0934 Cyg

57883.55986

V0981 Cyg

58073.33030

58073.33069

58073.33109

58073.33138

V1036 Cyg

58024.34756

V1048 Cyg 57989.37345

V1130 Cyg 57929.43178
0.0003 I CCD+Clear 272/203 Urbaník M.

0.0002 I CCD + Clear 246/93 Červinka L.

0.0004 II Photometer + V64/31 Persha G.

0.0003 I CCD+Clear 189/83 Urbaník M.

0.0014 I DSLR

217/24 Školník V.

Tamron LNS 44/300 + Canon

$600 \mathrm{D}$

Sky-Watcher NWT 150/750 + MII G2-8300

Sky-Watcher NWT 150/750 + MII G2-8300

Tamron 55-200@135mm, Atik $320 \mathrm{E}$ mono

Meade RCT 250/2500 + Optec SSP-5a

0.0009 II $\mathrm{CCD}+$ Clear $65 / 53 \quad$ Červinka L.

NWT 200/1000 + Atik 314L+

$0.0005 \mathrm{I} \quad \mathrm{CCD}+\mathrm{V} \quad 102 / 65 \quad$ Mazanec J.

$0.0003 \mathrm{I} \quad \mathrm{CCD}+\mathrm{R} \quad 118 / 74 \quad$ Mazanec J.

112/71 Mazanec J.

0.0005 I $\quad \mathrm{CCD}+\mathrm{B}$

0.0006 I $\quad \mathrm{CCD}+\mathrm{I}$

92/58 Mazanec J.

0.0004 I CCD+Clear 99/54 Urbaník M.

Sky-Watcher REF 80/520 + MII G2-8300

ORION, NWT 500/1500 + MII G2 402

ORION, NWT 500/1500 MII G2 402

ORION, NWT 500/1500 + MII G2 402

ORION, NWT 500/1500 + MII G2 402

Sky-Watcher NWT 150/750 + MII G2-8300

Sky-Watcher NWT 150/750 + MII G2-8300

Sky-Watcher NWT 150/750 + MII G2-8300 


\begin{tabular}{|c|c|c|c|c|c|}
\hline Star & & & & & \\
\hline HJD-2400000 & Error & I/II Method & Points & Observer & Technique \\
\hline 58036.31754 & 0.0001 & I $\quad$ CCD + Clear & $113 / 66$ & Urbaník M. & $\begin{array}{l}\text { Sky-Watcher NWT 150/750 + } \\
\text { MII G2-8300 }\end{array}$ \\
\hline
\end{tabular}

V1141 Cyg 57981.41356

V1171 Cyg 57951.55683

V1191 Cyg 58093.32975

V1256 Cyg 58027.37613

V1321 Cyg 57926.43063

V1425 Cyg 57984.47340

V1763 Cyg 57957.55355 57990.36753

V1815 Cyg 57705.34441

V1823 Cyg 57989.40626

V1901 Cyg 58028.34318

V2083 Cyg 57924.49504
0.0003 II CCD + Clear 105/62 Ehrenberger R. Quadruplet LNS 65/420+MII G2-8300

0.0004 II CCD+Clear 251/148 Bragagnolo U. Sky-Watcher REF 100/900 QHY9

0.0003 I CCD + Clear 293/189 Bragagnolo U. Sky-Watcher REF 100/900 + QHY9

0.0009 I $\quad$ CCD + Clear $\quad 77 / 52 \quad$ Urbaník M. Sky-Watcher NWT 150/750 + MII G2-8300

Sky-Watcher NWT 150/750 + MII G2-8300

LNS 58/200 + Canon 350 D

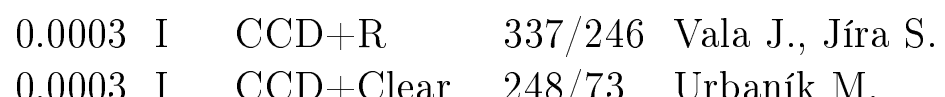

NWT 150/750 + MII G2-1600 Sky-Watcher NWT 150/750 + MII G2-8300

0.0005 I $\quad$ CCD + Clear $\quad 155 / 99$ Bragagnolo U. Sky-Watcher REF 100/900 + QHY9

0.0004 I CCD+Clear 69/48 Ehrenberger R. Quadruplet LNS 65/420+MII G2-8300

0.0008 I CCD+Clear 139/96 Urbaník M.

Sky-Watcher NWT 150/750+ MII G2-8300

Tamron SP LNS 62.5/500+ Canon 350 D 


\begin{tabular}{llllll}
\hline $\begin{array}{l}\text { Star } \\
\text { HJD-2400000 }\end{array}$ & Error & I/II Method & Points & Observer & Technique \\
\hline 58006.66358 & 0.0003 & I & Photometer+V70/31 & Persha G. & $\begin{array}{l}\text { Meade RCT 250/2500+ Optec } \\
\text { SSP-5a }\end{array}$
\end{tabular}

V2150 Cyg

58043.57919

V2154 Cyg

57951.40780

V2165 Cyg

57981.49997

V2282 Cyg

57996.45228

V2287 Cyg

57690.29072

57845.51061

V2364 Cyg

58017.36943

V2365 Cyg

57890.49934

V2366 Cyg

57895.43665

58030.37718

V2469 Cyg

57780.72725

57914.42001
0.0008 I $\quad$ Photometer + V69 $/ 40 \quad$ Persha G.

$0.0004 \mathrm{I} \quad \mathrm{CCD}+\mathrm{V} \quad 151 / 61 \quad$ Šuchaň J.

$0.0003 \mathrm{I} \quad \mathrm{CCD}+\mathrm{R} \quad 370 / 156 \quad$ Vrašták $\mathrm{M}$

0.0005 I $\quad$ CCD + Clear 118/35 Bragagnolo U.

Sky-Watcher REF 100/900 QHY9

0.0008 I CCD +Clear 85/53 Bragagnolo U. Sky-Watcher REF 100/900 QHY9

0.0003 I CCD +Clear 391/164 Urbaník M. Sky-Watcher NWT 150/750 + MII G2-8300

0.0002 II CCD + Clear $\quad 180 / 90 \quad$ Bragagnolo U. Sky-Watcher REF 100/900 + QHY9

0.0002 I $\quad$ CCD +B $\quad 166 / 72 \quad$ Vrašták M.

NWT 280/1500+MII G2-1600, $80 / 400+$ G1-0301

0.0011 II CCD +Clear 142/34 Červinka L. R102/500 + Atik 320E mono, $0.5 \mathrm{x} \mathrm{FC}$

0.0001 I CCD + Clear 330/204 Audejean M. 0.32-m f/6 Newtonian reflector

$+\mathrm{CCD}$

0.0007 I $\quad$ CCD + Clear $37 / 28 \quad$ Walter F.

0.0003 I CCD+Clear 206/83 Urbaník M.
MARK SCT 406/4060, SBIG ST10XME

Sky-Watcher NWT 150/750 + MII G2-8300 


\begin{tabular}{|c|c|c|c|c|c|}
\hline Star & & & & & \\
\hline HJD-2400000 & Error & I/II Method & Points & Observer & Technique \\
\hline 57948.48505 & 0.0002 & I $\quad$ CCD + Clear & $202 / 174$ & Urbaník M. & $\begin{array}{l}\text { Sky-Watcher NWT 150/750 + } \\
\text { MII G2-8300 }\end{array}$ \\
\hline
\end{tabular}

V2477 Cyg

57901.47661

57980.53426

57980.53442

V2490 Cyg

57988.55095

V2497 Cyg

57981.43515

V2544 Cyg 57973.41873

V2545 Cyg 57991.48871

V2546 Cyg 57991.45610

57991.45637

V2549 Cyg 57689.32176

57991.48524

57991.48529

V2628 Cyg 57964.40806

$\begin{array}{lllll}0.0002 \text { I } & \text { DSLR } & 210 / 167 \text { Školník V. } & \begin{array}{l}\text { Tamron LNS } 44 / 300+\text { Canon } \\ 600 \mathrm{D}\end{array} \\ 0.0003 \text { I } & \text { DSLR } & 45 / 20 & \text { Walter F. } & \begin{array}{l}\text { Tamron SP LNS } 62,5 / 500+ \\ \text { Canon 350D }\end{array} \\ 0.0004 \text { I } & \text { DSLR } & 48 / 22 & \text { Walter F. } & \begin{array}{l}\text { Tamron SP LNS } 62,5 / 500+ \\ \text { Canon 350D }\end{array}\end{array}$

0.0003 I CCD + Clear 198/80 Bragagnolo U. Sky-Watcher REF 100/900 + QHY9

0.0002 I CCD+Clear 311/159 Urbaník M.

Sky-Watcher NWT 150/750+ MII G2-8300

Sky-Watcher NWT 254/1200 + MII G2-402

0.0019 II DSLR $\quad 129 / 96 \quad$ Walter F.

Tamron SP LNS $62.5 / 500+$ Canon 350 D

Tamron SP LNS 62.5/500 + Canon 350 D

Tamron SP LNS 62.5/500+

Canon 350 D

138/80 Walter F.

138/80 Walter F.

Sky-Watcher REF 100/900 + QHY9

MARK SCT 406/4060, SBIG ST10XME

Tamron SP LNS 62.5/500 + Canon 350 D

Sky-Watcher NWT 150/750 + MII G2-8300 


\begin{tabular}{|c|c|c|c|c|c|c|c|}
\hline \multicolumn{8}{|l|}{ Star } \\
\hline HJD-2400000 & Error & I/II & Method & Points & Observer & & Technique \\
\hline 57996.52924 & 0.0016 & I & CCD + Clear & $352 / 297$ & Urbaník M. & & $\begin{array}{l}\text { Sky-Watcher NWT 150/750 + } \\
\text { MII G2-8300 }\end{array}$ \\
\hline \multicolumn{8}{|l|}{ V2703 Cyg } \\
\hline 57994.38517 & 0.0012 & I & CCD + Clear & $295 / 109$ & Urbaník M. & & $\begin{array}{l}\text { Sky-Watcher NWT 150/750 + } \\
\text { MII G2-8300 }\end{array}$ \\
\hline \multicolumn{8}{|c|}{ VSX J200002.9+522850 Cyg } \\
\hline 57948.45549 & 0.0007 & I & CCD + Clear & $207 / 135$ & Urbaník M. & & $\begin{array}{l}\text { Sky-Watcher NWT 150/750 + } \\
\text { MII G2-8300 }\end{array}$ \\
\hline \multicolumn{8}{|l|}{ WZ Cyg } \\
\hline 57973.48099 & 0.0002 & II & $\mathrm{CCD}+$ Clear & $198 / 63$ & $\begin{array}{l}\text { Walter } \\
\text { Mokrý A. }\end{array}$ & F., & $\begin{array}{lcc}\text { CZ } & \text { Kometensucher } & \text { REF } \\
200 / 1370+\text { SBIG ST7 } & \end{array}$ \\
\hline 57973.48470 & 0.0004 & II & DSLR & $242 / 97$ & $\begin{array}{l}\text { Walter } \\
\text { Mokrý A. }\end{array}$ & F., & $\begin{array}{l}\text { CZ Mirotar LNS } 180 / 1000+ \\
\text { Canon 350D }\end{array}$ \\
\hline
\end{tabular}

AL Del 57956.42356

0.0012 I $\quad$ CCD +Clear 68/28 Ehrenberger R.

Quadruplet LNS 65/420+MII G2-8300

FO Del

58031.34809

0.0002 I CCD + Clear 183/64 Audejean M.

0.32-m f/6 Newtonian reflector $+\mathrm{CCD}$

KO Del

58027.41911

0.0002 II CCD+Clear 151/116 Mašek M.

NWT 150/600 + CCD MII G2-1600 + comacorector

MZ Del

57995.43881

0.0021 I $\quad C C D+$ Clear $71 / 29 \quad$ Hladík B.

LNS 38/135 + ATIK 320E + MuniWin

OW Del

57967.44208

0.0001 I CCD +Clear 210/103 Nosál P.

REF $102 / 500+$ ATIK 16 IC mono

OZ Del

57966.39218

0.0011 I $\quad$ CCD + Clear $\quad 296 / 50 \quad$ Nosál P.

REF $102 / 500+$ ATIK 16 IC mono

PS Del 


\begin{tabular}{|c|c|c|c|c|c|c|}
\hline \multicolumn{7}{|l|}{ Star } \\
\hline HJD-2400000 & Error & $\mathrm{I} / \mathrm{II}$ & Method & Points & Observer & Technique \\
\hline 57992.40004 & 0.0002 & $\mathrm{I}$ & CCD+Clear & $223 / 103$ & Nosál P. & $\begin{array}{l}\text { REF } 102 / 500+\text { ATIK } 16 \text { IC } \\
\text { mono }\end{array}$ \\
\hline $\begin{array}{l}\text { VESPA V39 De } \\
57599.51117\end{array}$ & 0.0002 & I & CCD + Clear & $213 / 170$ & Lomoz F. & SNT $254 / 1016+$ G2-8300 \\
\hline $\begin{array}{l}\text { VESPA V47 D } \\
57599.45183\end{array}$ & 0.0009 & I & $\mathrm{CCD}+$ Clear & $213 / 84$ & Lomoz F. & STN 254/1016+G2-8300 \\
\hline VSX J202927.1 & Del & & & & & \\
\hline $\begin{array}{l}57964.39907 \\
57964.54894\end{array}$ & $\begin{array}{l}0.0001 \\
0.0001\end{array}$ & $\begin{array}{l}\text { II } \\
\text { I }\end{array}$ & $\begin{array}{l}\text { CCD }+ \text { Clear } \\
\text { CCD }+ \text { Clear }\end{array}$ & $\begin{array}{l}328 / 43 \\
328 / 249\end{array}$ & $\begin{array}{l}\text { Lomoz F. } \\
\text { Lomoz F. }\end{array}$ & $\begin{array}{l}\text { Newton } 300 / 1200+\text { ST2000XM } \\
\text { NWT } 300 / 1200+\quad \text { CCD- } \\
\text { ST2000XM }\end{array}$ \\
\hline
\end{tabular}

VSX J202950.3+063344 Del

57964.45335 0.0003 II $\quad$ CCD + Clear $334 / 121$ Lomoz F.

NWT $300 / 1200+$ CDDST2000XM

VSX J203145.6+061349 Del

57964.47233 $\quad 0.0007$ I $\quad$ CCD + Clear $\quad 388 / 196$ Lomoz F.

STN 254/1016+CCD-G2-8300

ASAS J044625-6620.8 Dor

$58060.66747 \quad 0.0019$ I $\quad$ CCD $+B \quad 76 / 45 \quad$ Mašek M.

FRAM, Nikkor LNS 106/300 + G4-16000

ASAS J045948-6508.4 Dor

58060.67728

$0.0011 \mathrm{I} \quad \mathrm{CCD}+\mathrm{B} \quad 77 / 49 \quad$ Mašek M.

FRAM, Nikkor LNS 106/300 + G4-16000

ASAS J054000-6828.7 Dor

58060.71874

0.0007 II $\mathrm{CCD}+\mathrm{B} \quad 75 / 62 \quad$ Mašek M.

FRAM, Nikkor LNS 106/300 + G4-16000

RW Dor

58060.62672

0.0001 II CCD +Clear 150/61 Mašek M.

FRAM, Meade SCT 300/3000 + MII G2-1600

AR Dra

57760.33919

0.0002 I CCD +Clear 463/389 Medulka T.

REF 90/500 + MII G1-300

AU Dra 


\begin{tabular}{|c|c|c|c|c|c|}
\hline Star & & & & & \\
\hline HJD-2400000 & Error & I/II Method & Points & Observer & Technique \\
\hline 57923.47627 & 0.0005 & II $\quad \mathrm{CCD}+\mathrm{V}$ & $55 / 39$ & Lehky M. & $\begin{array}{l}\text { JST, NWT } 400 / 2000+\text { MII } \\
\text { G2-1600 + BVRcIc }\end{array}$ \\
\hline 57923.47649 & 0.0003 & $\mathrm{CCD}+\mathrm{R}$ & $55 / 41$ & Lehky M. & $\begin{array}{l}\text { JST, NWT } 400 / 2000+\text { MII } \\
\text { G2- } 1600+\text { BVRcIc }\end{array}$ \\
\hline
\end{tabular}

AX Dra

57845.35236

BE Dra

57969.57095

BW Dra

57798.40598

BX Dra

57902.54271

57902.54285

\section{EF Dra}

57481.44863

57845.49121

57884.50313

57884.50327

57884.50342

57884.50354

FU Dra

57780.45685

57780.61006

57796.40674

57838.58045

57868.33232

57868.33242

57868.48588
0.0006 II CCD+Clear 124/89 Vrašták M.

RF 80/400+G1-0300

0.0002 I CCD + Clear 263/174 Bragagnolo U.

Sky-Watcher REF 100/900 QHY9

0.0001 I $\quad$ CCD + Clear $\quad 176 / 77$ Červinka L.

$\mathrm{R} 102 / 500+$ Atik 314L+

\begin{tabular}{|c|c|c|}
\hline $0.0005 \mathrm{I}$ & $\mathrm{CCD}+\mathrm{R}$ & $52 / 40$ \\
\hline $.0006 \mathrm{I}$ & $\mathrm{CCD}+\mathrm{I}$ & $50 / 39$ \\
\hline
\end{tabular}

HK25, NWT 250/1000 SBIG ST-7 + BVRcIc HK25, NWT 250/1000 + SBIG ST-7 + BVRcIc

$\begin{array}{lllll}0.0009 & \text { I } & \text { DSLR } & 133 / 64 & \text { Sergey I. } \\ 0.0003 & \text { II } & \text { CCD }+ \text { Clear } & 179 / 88 & \text { Vrašták M. } \\ 0.0003 & \text { II } & \text { CCD }+ \text { R } & 95 / 60 & \text { Lehky M. } \\ 0.0005 & \text { II } & \text { CCD }+B & 94 / 61 & \text { Lehky M. } \\ 0.0003 & \text { II } & \text { CCD }+ \text { I } & 95 / 60 & \text { Lehky M. } \\ 0.0003 & \text { II } & \text { CCD }+ \text { V } & 95 / 61 & \text { Lehky M. }\end{array}$

Newton 200 mm (1:5) + Canon $400 \mathrm{D}$

RF $80 / 400+$ G1-0300

JST, NWT 400/2000 + MII

G2-1600 + BVRcIc

JST, NWT 400/2000 + MII

G2-1600 + BVRcIc

JST, NWT 400/2000 + MII

G2-1600 + BVRcIc

JST, NWT 400/2000 + MII

G2-1600 + BVRcIc

\begin{tabular}{|c|c|c|c|c|}
\hline 0.0001 II & CCD +Clear & $82 / 90$ & Červinka L. & $\mathrm{R} 102 / 500+$ Atik $314 \mathrm{~L}+$ \\
\hline $0.0001 \mathrm{I}$ & CCD +Clear & $682 / 480$ & Cervinka L. & $\mathrm{R} 102 / 500+$ Atik $314 \mathrm{~L}+$ \\
\hline 0.0001 II & CCD + Clear & $97 / 48$ & Medulka T. & REF $90 / 500+$ MII G1-300 \\
\hline $0001 \mathrm{I}$ & $\mathrm{CCD}+$ & $77 / 50$ & $\begin{array}{l}\text { Mašek M., Tyl- } \\
\text { šar M. }\end{array}$ & $\begin{array}{l}\text { NWT 250/1000 + CCD MI } \\
\text { G2-8300 }\end{array}$ \\
\hline $0.0001 \mathrm{I}$ & $\mathrm{CCD}+\mathrm{R}$ & $218 / 38$ & Šmelcer L. & $\begin{array}{l}\text { Celestron SCT } 355 / 2460 \\
\text { MII G2-1600 }\end{array}$ \\
\hline $0.0001 \mathrm{I}$ & $\mathrm{CCD}+\mathrm{V}$ & $209 / 29$ & Šmelcer L. & $\begin{array}{l}\text { Celestron SCT } 355 / 2460 \\
\text { MII G2-1600 }\end{array}$ \\
\hline .0001 & $\mathrm{CCD}+\mathrm{R}$ & & & $\begin{array}{l}\text { Celestron SCT } 355 / 2460 \\
\text { MII G2-1600 }\end{array}$ \\
\hline
\end{tabular}




\begin{tabular}{llllllll}
\hline Star & & & & & & & \\
HJD-2400000 & Error & I/II Method & Points & Observer & Technique & \\
\hline 57868.48594 & 0.0001 & II & CCD + V & $209 / 145$ & Śmelcer L. & $\begin{array}{l}\text { Celestron SCT } \\
\text { MII G2-1600 }\end{array}$ &
\end{tabular}

GM Dra

57919.40445

GQ Dra

57923.46629

HZ Dra

57434.34255

IV Dra

57954.41174

LSPM J1112+7626

Dra

57735.50195

LZ Dra

57884.37159

57884.37192

57884.37203

57884.37223

57989.49180

NN Dra

57473.37585

57840.36267

57854.33544

57854.33566

57854.33574

57874.43566
0.0004 II CCD + Clear $\quad 160 / 75$ Červinka L.

0.0003 I DSLR $\quad 108 / 41 \quad$ Školník V.

0.0004 I CCD + Clear 509/225 Jacobsen J.

EQ6, $80 \mathrm{~mm}$ refractor, $\mathrm{mx} 716$

320 E mono

Tamron LNS 44/300 + Canon 600D

MARK SCT 406/4060, SBIG ST10XME

0.0003 I $\quad$ CCD + Clear $\quad 99 / 62$ Walter F.

257/132 Smolka M.

NWT $\quad 250 / 1000$

$314 \mathrm{~L}++$ MPCC

ATIK

0.0003 II $\mathrm{CCD}+\mathrm{R}$

0.0002 I $\quad$ CCD $+\mathrm{I} \quad 33 / 19 \quad$ Auer R. F.

NWT 300/1410 MI G2-1600

0.0002 I $\quad \mathrm{CCD}+\mathrm{R} \quad 33 / 19 \quad$ Auer R. F.

NWT 300/1410 MI G2-1600

0.0007 I $\quad \mathrm{CCD}+\mathrm{B} \quad 24 / 17$ Auer R. F.

NWT 200/810 MI G2-1600

0.0003 I $\quad \mathrm{CCD}+\mathrm{V} \quad 33 / 19 \quad$ Auer R. F.

NWT 300/1410 MI G2-1600

0.0001 I CCD + Clear 153/81 Bragagnolo U.

Sky-Watcher REF 100/900+ QHY9

0.0002 I $\quad$ CCD + Clear $\quad 191 / 47 \quad$ Jacobsen J.

EQ6, $100 \mathrm{~mm}$ refractor, Atik 314

0.0001 II $\quad \mathrm{CCD}+$ Clear $\quad 141 / 62 \quad$ Ehrenberger R.

Quadruplet LNS 65/420+MII G2-8300

0.0002 I $\quad \mathrm{CCD}+\mathrm{V} \quad 37 / 11 \quad$ Vrašták M.

NWT 280/1500+MII G2-1600, $80 / 400+$ G1-0301

0.0001 I $\quad \mathrm{CCD}+\mathrm{I} \quad 35 / 12 \quad$ Vrašták M.

NWT 280/1500+MII G2-1600, $80 / 400+$ G1-0301

$0.0001 \mathrm{I} \quad \mathrm{CCD}+\mathrm{R} \quad 38 / 12 \quad$ Vrašták M.

NWT 280/1500+MII G2-1600, $80 / 400+$ G1-0301

HK25, NWT 250/1000 + SBIG ST-7 + BVRcIc 


\begin{tabular}{|c|c|c|c|c|c|c|c|c|}
\hline Star & & & & & & & & \\
\hline HJD-2400000 & Error & $\mathrm{I} / \mathrm{I}$ & Method & Points & Observer & Technique & & \\
\hline 57891.46660 & 0.0002 & II & $\mathrm{CCD}+\mathrm{R}$ & $86 / 57$ & Śmelcer L. & $\begin{array}{l}\text { Celestron SCT } \\
\text { MII G2-1600 }\end{array}$ & $355 / 2460$ & \\
\hline 57891.46697 & 0.0005 & II & $\mathrm{CCD}+\mathrm{V}$ & $59 / 43$ & Šmelcer L. & $\begin{array}{l}\text { Celestron SCT } \\
\text { MII G2-1600 }\end{array}$ & $355 / 2460$ & + \\
\hline 57892.42600 & 0.0001 & I & $\mathrm{CCD}+\mathrm{V}$ & $70 / 39$ & Śmelcer L. & $\begin{array}{l}\text { Celestron SCT } \\
\text { MII G2-1600 }\end{array}$ & $355 / 2460$ & + \\
\hline 57892.42610 & 0.0001 & I & $\mathrm{CCD}+\mathrm{R}$ & $70 / 39$ & Šmelcer L. & $\begin{array}{l}\text { Celestron SCT } \\
\text { MII G2-1600 }\end{array}$ & $355 / 2460$ & + \\
\hline 57902.40797 & 0.0001 & I & $\mathrm{CCD}+\mathrm{R}$ & $220 / 51$ & Šmelcer L. & $\begin{array}{l}\text { Celestron SCT } \\
\text { MII G2-1600 }\end{array}$ & $355 / 2460$ & + \\
\hline 57906.43967 & 0.0001 & II & $\mathrm{CCD}+\mathrm{R}$ & $205 / 116$ & Šmelcer L. & $\begin{array}{l}\text { Celestron SCT } \\
\text { MII G2-1600 }\end{array}$ & $355 / 2460$ & + \\
\hline 57925.44381 & 0.0001 & I & $\mathrm{CCD}+\mathrm{R}$ & $155 / 93$ & Šmelcer L. & $\begin{array}{l}\text { Celestron SCT } \\
\text { MII G2-1600 }\end{array}$ & $355 / 2460$ & + \\
\hline
\end{tabular}

NSVS 2690221 Dra

57874.32407

57874.47890

NT Dra

57853.45846

NX Dra

57883.45030

OO Dra

57823.62126

OQ Dra

57408.34757

57854.34526

OX Dra

57689.54976

PR Dra

57715.60478

0.002 I $\quad \mathrm{CCD}+$ Clear

212/115 Audejean M.

0.32-m f/6 Newtonian reflector $+\mathrm{CCD}$

Skywatcher NWT 200/1000 + SBIG ST-9E + comacorre

HK25， NWT 250/1000 + SBIG ST-7 + BVRcIc

HK25， NWT 250/1000 + SBIG ST-7 + BVRcIc

0.0001 I $\quad$ CCD + Clear $360 / 151$ Trnka J.

0.0004 I CCD + Clear 286/114 Bragagnolo U.

Sky-Watcher REF 100/900 + QHY9

0.0005 I CCD + Clear 297/166 Jacobsen J.

0.0003 I CCD + Clear 191/102 Urbaník M.

EQ6, $100 \mathrm{~mm}$ refractor, Atik 314

Sky-Watcher NWT 150/750 + MII G2-8300

PT Dra
0.0001 I CCD +Clear 211/91 Medulka T. REF 90/500 + MII G1-300

0.0005 II CCD +Clear 197/87 Medulka T. REF 90/500 + MII G1-300 


\begin{tabular}{|c|c|c|c|c|c|}
\hline \multicolumn{6}{|l|}{ Star } \\
\hline HJD-2400000 & Error & I/II Method & Points & Observer & Technique \\
\hline 57799.47566 & 0.0005 & II $\quad \mathrm{CCD}+\mathrm{V}$ & $137 / 28$ & Lehky M. & $\begin{array}{l}\text { JST, NWT } 400 / 2000+\text { MII } \\
\text { G2-1600 + BVRcIc }\end{array}$ \\
\hline 57799.47639 & 0.0004 & $\mathrm{CCD}+\mathrm{R}$ & $144 / 26$ & Lehky M. & $\begin{array}{l}\text { JST, NWT } 400 / 2000+\text { MII } \\
\text { G2-1600 + BVRcIc }\end{array}$ \\
\hline 57799.60930 & 0.0004 & $\mathrm{CCD}+\mathrm{V}$ & $137 / 93$ & Lehky M. & $\begin{array}{l}\text { JST, NWT } 400 / 2000+\text { MII } \\
\text { G2-1600 + BVRcIc }\end{array}$ \\
\hline 57799.61013 & 0.0002 & $\mathrm{CCD}+\mathrm{R}$ & $144 / 93$ & Lehky M. & $\begin{array}{l}\text { JST, NWT } 400 / 2000+\text { MII } \\
\text { G2- } 1600+\text { BVRcIc }\end{array}$ \\
\hline
\end{tabular}

RZ Dra

57845.62785

TZ Dra

58095.19710

V0342 Dra

57435.33628

V0349 Dra

57924.43519

57995.38737

V0357 Dra

57434.32423

57969.39316

V0362 Dra

57392.35566

57902.43096

V0372 Dra

57991.35160

V0414 Dra

57988.37561
0.001 II CCD + Clear 497/459 Červinka L.

$\mathrm{R} 102 / 500+$ Atik 314L+

0.0003 I CCD + Clear $239 / 57$ Walter F.

$\mathrm{CZ} \quad$ Kometensucher $200 / 1370+$ SBIG ST7

0.0005 I CCD + Clear 264/105 Jacobsen J.

EQ6, $100 \mathrm{~mm}$ refractor, Atik 314

0.0003 I CCD+Clear 183/30 Bragagnolo U. Sky-Watcher REF 100/900 QHY9

0.0002 I CCD+Clear 203/101 Ehrenberger R. Quadruplet LNS 65/420+MII G2-8300

0.0029 I CCD + Clear 255/59 Jacobsen J. $\quad$ EQ6, 100 mm refractor, Atik 314

0.0003 I CCD +Clear 253/102 Bragagnolo U. Sky-Watcher REF 100/900 QHY9

EQ6, $80 \mathrm{~mm}$ refractor, SXVRH16

NWT 240/1200+MII G2-1600, $80 / 400+$ G1-0300

0.0001 I CCD +Clear 229/133 Bragagnolo U. Sky-Watcher REF 100/900 + QHY9 


\begin{tabular}{|c|c|c|c|c|c|}
\hline Star & & & & & \\
\hline HJD-2400000 & Error & I/II Method & Points & Observer & Technique \\
\hline 58024.49515 & 0.0005 & I $\quad$ CCD + Clear & $250 / 179$ & Bragagnolo U & $\begin{array}{l}\text { Sky-Watcher REF } 100 / 900+ \\
\text { QHY9 }\end{array}$ \\
\hline
\end{tabular}

V0436 Dra

$\begin{array}{lllllll}57902.39364 & 0.0003 & \text { II } & \text { CCD }+ \text { R } & 153 / 35 & \text { Hanžl D. } & \text { DATEL, NWT 200/800 + MII } \\ 57988.43151 & 0.0006 & \text { I } & \text { CCD }+ \text { R } & 185 / 61 & \text { Hanžl D. } & \text { DATEL, NWT 200/800 + MII } \\ & & & & & & \text { G2 8300 }\end{array}$

V0438 Dra

58054.32531

V0445 Dra

57884.35514

57884.51636

57901.39319

57901.55437

V0449 Dra

57434.38665

58009.48631

WX Dra

57829.54293

Z Dra

57739.24556

57739.24590
0.0004 I $\quad$ CCD + Clear $\quad 386 / 143$ Bragagnolo U.

Sky-Watcher REF 100/900 + QHY9

0.0004 II $\quad$ CCD + R $\quad 172 / 13$ Hanžl D.

$0.0003 \mathrm{I} \quad \mathrm{CCD}+\mathrm{R} \quad$ 172/119 Hanžl D.

0.0003 II $\mathrm{CCD}+\mathrm{R} \quad 168 / 42 \quad$ Hanžl D.

0.0003 I $\quad \mathrm{CCD}+\mathrm{R} \quad$ 168/150 Hanžl D.

0.0004 I CCD +Clear 247/144 Jacobsen J.

0.0005 I CCD + Clear 167/86 Bragagnolo U.

0.0001 I CCD +Clear 319/185 Trnka J.

$0.0001 \mathrm{I} \quad \mathrm{CCD}+\mathrm{R} \quad 144 / 42 \quad \check{\text { Śmelcer } \mathrm{L} .}$

0.0001 I $\quad \mathrm{CCD}+\mathrm{V} \quad 148 / 45 \quad$ Šmelcer L.
DATEL, NWT 200/800 + MII G2 8300

DATEL, NWT 200/800 + MII

G2 8300

DATEL, NWT 200/800 + MII

G2 8300

DATEL, NWT 200/800 + MII

G2 8300

EQ6, $80 \mathrm{~mm}$ refractor, SXVRH16

Sky-Watcher REF 100/900 QHY9

Skywatcher NWT 200/1000 + SBIG ST-9E + comacorre

Celestron SCT 355/2460 MII G2-1600

Celestron SCT 355/2460 + MII G2-1600

ASAS J210245+1159.9 Equ

57980.36172

0.0004 I CCD + Clear 272/55 Lomoz F.

SNT 254/1016+G2-8300

CSS J210116.1+115236 Equ 


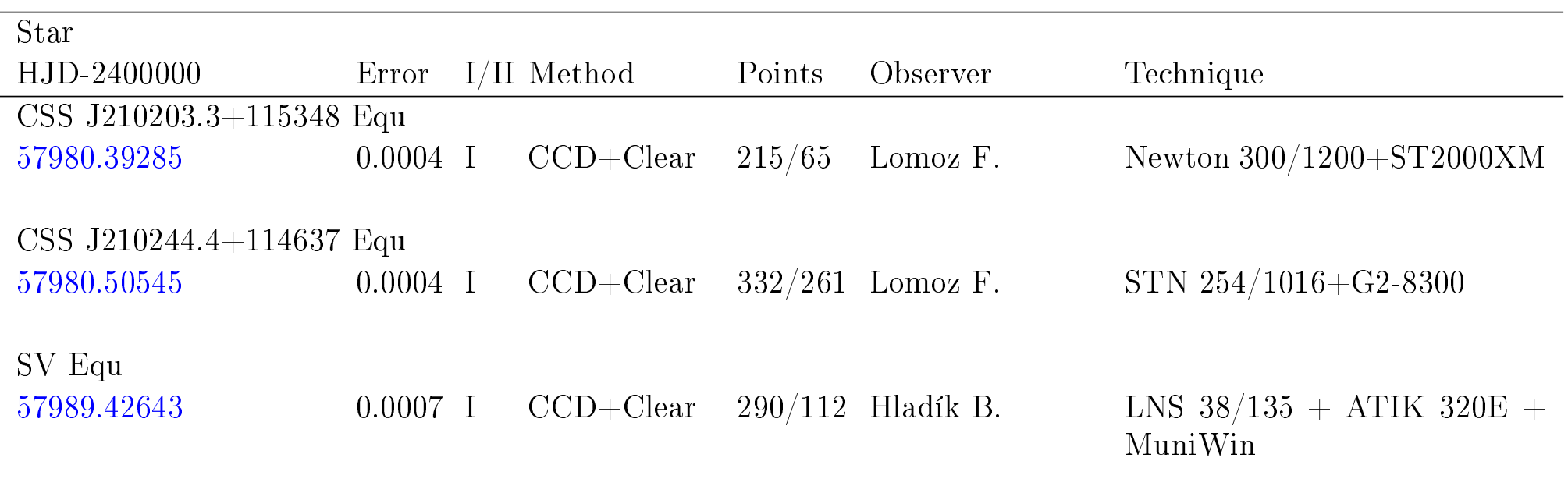

FX Eri

57747.73371

0.0004 II $\quad \mathrm{CCD}+\mathrm{R} \quad$ 178/87 Mašek M.

FRAM, Nikkor LNS 106/300

+ G4- 16000

GH Eri

57747.74382

$0.0001 \mathrm{I} \quad \mathrm{CCD}+\mathrm{R} \quad$ 179/101 Mašek M.

FRAM, Nikkor LNS 106/300 + G4-16000

GSC 07030-01448 Eri 57715.60894

$0.001 \quad \mathrm{I} \quad \mathrm{CCD}+\mathrm{R} \quad 102 / 67 \quad$ Mašek M.

FRAM, Nikkor LNS 106/300 + G4-16000

KZ Eri

58027.53655

0.0001 I CCD + Clear 80/44 Mašek M.

NWT 150/600 + CCD MII G2-1600 + comacorector

NSVS 17651112 Eri

57715.61818

0.0007 I $\quad \mathrm{CCD}+\mathrm{R} \quad$ 109/78 Mašek M.

FRAM, Nikkor LNS 106/300 + G4-16000

ASAS J063016+1835.0 Gem

$57726.58103 \quad 0.0002$ I $\quad$ CCD + Clear $\quad 330 / 166$ Trnka J.

Skywatcher NWT 200/1000 + SBIG ST-9E + comacorre

ASAS J063047+2728.4 Gem

57751.25581

0.0014 I DSLR

229/47 Červinka L.

$\mathrm{R} 102 / 500+$ Canon 1000D

ASAS J064543+1340.0 Gem

57752.37082

0.0003 I DSLR $\quad 163 / 52 \quad$ Nosál P.

NWT 150/600 + Canon 20D + MPCC mk.III 


\begin{tabular}{|c|c|c|c|c|c|}
\hline Star & & & & & \\
\hline HJD-2400000 & Error & I/II Method & Points & Observer & Technique \\
\hline 58144.27791 & 0.0005 & I $\quad$ CCD + Clear & $276 / 134$ & Urbaník M. & $\begin{array}{l}\text { Sky-Watcher NWT 150/750 + } \\
\text { MII G2-8300 }\end{array}$ \\
\hline
\end{tabular}

ASAS J072656+1945.7 Gem

$\begin{array}{lllll}57780.58172 & 0.0004 \mathrm{I} \quad \mathrm{CCD}+\text { Clear } & 109 / 89 \quad \text { Walter F. } & \begin{array}{l}\text { MARK SCT } 406 / 4060, \text { SBIG } \\ \text { ST10XME }\end{array}\end{array}$

CzeV656 Gem

57781.38375

0.0006 I $\quad$ CCD + Clear $375 / 201$ Šmelcer L.

Celestron SCT 280/1765

MII G2-4000

EG Gem

57783.47998

0.0009 II CCD + Clear $\quad 104 / 37$ Walter F .

MARK SCT 406/4060, SBIG ST10XME

FG Gem

57783.26851

0.0022 II $\quad$ CCD + Clear $\quad 421 / 98$ Červinka L.

$\mathrm{R} 102 / 500+$ Atik 314L +

GW Gem

57826.48032

0.0001 I CCD+Clear 191/94 Urbaník M.

Sky-Watcher NWT 150/750 + MII G2-8300

IV Gem

57752.47490

0.0022 II $\quad \mathrm{CCD}+\mathrm{I}$

63/29 Vrašták M

NWT 280/1500+MII G2-1600, $80 / 400+$ G1-0300

57752.47687

0.0016 II $\mathrm{CCD}+\mathrm{R}$

61/29 Vrašták M

57752.48124

$0.0012 \quad$ II $\quad \mathrm{CCD}+\mathrm{V} \quad 63 / 29 \quad$ Vrašták M

NWT 280/1500+MII G2-1600, $80 / 400+$ G1-0300

NWT 280/1500+MII G2-1600, $80 / 400+$ G1-0300

KK Gem

57726.58425

0.0002 I CCD + Clear $\quad 305 / 154$ Trnka J.

Skywatcher NWT 200/1000 + SBIG ST-9E + comacorre

KV Gem

57777.25591

0.0002 II CCD + Clear $327 / 75 \quad$ Šmelcer L.

Celestron SCT 280/1765

MII G2-4000

57780.30237

0.0001 I CCD + Clear 456/131 Šmelcer L.

Celestron SCT 280/1765 +

MII G2-4000

57780.48281

0.0001 II CCD + Clear $456 / 358$ Šmelcer L.

Celestron SCT 280/1765 +

MII G2-4000

57781.37856

0.0001 I $\quad$ CCD + Clear $344 / 188$ Šmelcer L.

Celestron SCT 280/1765 + MII G2-4000 


\begin{tabular}{|c|c|c|c|c|c|}
\hline $\begin{array}{l}\text { Star } \\
\text { HJD-2400000 }\end{array}$ & Error & I/II Method & Points & Observer & Technique \\
\hline 58178.38737 & 0.0002 & I $\quad \mathrm{CCD}+\mathrm{R}$ & $103 / 59$ & Mašek M. & $\begin{array}{l}\text { NWT } 150 / 600+\text { CCD MII } \\
\text { G2-1600+ comacorector }\end{array}$ \\
\hline
\end{tabular}

QW Gem

57739.35588

57739.35655

57739.35669

57739.35694

SvkV107 Gem

57715.55690

57780.34564

58132.45111

58134.42052

SX Gem

58181.30273

$\begin{array}{lllll}0.0004 & \text { II } & \text { CCD }+\mathrm{R} & 87 / 48 & \text { Lehky M. } \\ 0.0003 \text { II } & \text { CCD }+ \text { V } & 83 / 48 & \text { Lehky M. } \\ 0.0004 & \text { II } & \text { CCD }+ \text { I } & 89 / 48 & \text { Lehky M. } \\ 0.0003 \text { II } & \text { CCD }+ \text { B } & 77 / 49 & \text { Lehky M. }\end{array}$

0.0006 II CCD+Clear 101/74 Urbaník M.

0.0014 I CCD+Clear 131/96 Urbaník M.

0.0006 I CCD+Clear 174/125 Urbaník M.

0.0008 I CCD+Clear 92/69 Urbaník M.

0.0004 I CCD+Clear 150/67 Urbaník M.
JST, NWT 400/2000 + MII

G2-1600 + BVRcIc

JST, NWT 400/2000 + MII G2-1600 + BVRcIc

JST, NWT 400/2000 + MII

G2-1600 + BVRcIc

JST, NWT 400/2000 + MII G2-1600 + BVRcIc

Sky-Watcher NWT 150/750+ MII G2-8300

Sky-Watcher NWT 150/750 + MII G2-8300

Sky-Watcher NWT 150/750 + MII G2-8300

Sky-Watcher NWT 150/750

MII G2-8300

Sky-Watcher REF 80/520 + MII G2-8300

Sky-Watcher REF 80/520 + MII G2-8300

Sky-Watcher NWT 254/1200 + MII G2-402

Sky-Watcher NWT 254/1200 + MII G2-402

V0345 Gem 57065.40694

0.0006 II $\mathrm{CCD}+\mathrm{V}$

$47 / 25$

Gudmundsson S.

$300 \mathrm{~mm}$ LX200 SCT/SBIG STL11k CCD 


\begin{tabular}{|c|c|c|c|c|c|}
\hline Star & & & & & \\
\hline HJD-2400000 & Error & I/II Method & Points & Observer & $\begin{array}{l}\text { Technique } \\
\text { BFF 102/500 + ATIK 16IC }\end{array}$ \\
\hline 58149.35922 & 0.001 & I $\quad$ CCD+Clear & $193 / 93$ & Nosál' P. & $\begin{array}{l}\text { REF } 102 / 500+\text { ATIK 16IC } \\
\text { mono }\end{array}$ \\
\hline
\end{tabular}

V0380 Gem 58101.40396 58101.40403

V0388 Gem 57780.26887

V0389 Gem 57760.34729

V0390 Gem 57743.58972

V0401 Gem 57715.50550 58104.49158

V0402 Gem 57780.35247 57800.31479 57800.31558 57800.31571 57800.31577 57800.31590 58095.56049 58149.25923 58149.46112
0.0001 II $\mathrm{CCD}+\mathrm{R}$ 0.0001 II $\mathrm{CCD}+\mathrm{V}$ $55 / 19$ $55 / 19$

Vrašták M. Vrašták M.

$0.0001 \mathrm{I} \quad \mathrm{CCD}+\mathrm{R}$ 218/78 Šmelcer L. 0.0003 I $\quad C C D+V \quad 124 / 66 \quad$ Suchaň J. 0.0002 I CCD + Clear $\quad 135 / 64$ Lehky M.

0.0004 I CCD+Clear 99/34 Urbaník M. 0.0005 I CCD+Clear 139/101 Nosál P.

0.0002 I CCD +Clear 133/104 Urbaník M. $0.0001 \mathrm{I} \quad \mathrm{CCD}+\mathrm{V} \quad 178 / 77 \quad$ Mazanec J. $0.0002 \mathrm{I} \quad \mathrm{CCD}+\mathrm{I} \quad 163 / 67 \quad$ Mazanec J. $0.0001 \mathrm{I} \quad \mathrm{CCD}+\mathrm{R} \quad 164 / 70 \quad$ Mazanec J. 0.0002 I CCD+Clear 271/124 Urbaník M. $0.0002 \mathrm{I} \quad \mathrm{CCD}+\mathrm{B} \quad 178 / 78 \quad$ Mazanec J. 0.0003 II CCD+Clear 171/100 Urbaník M. 0.0004 I DSLR 203/18 Nosál P. 0.0003 II DSLR
NWT 355/1600+MII G2-1600 NWT 355/1600+MII G2-1600

Celestron SCT 355/2460 + MII G2-1600

Sky-Watcher NWT 250/1200 + Algol 0402

HK25, NWT 250/1000 + SBIG ST-7 + C

Sky-Watcher NWT 150/750 + MII G2-8300

REF $102 / 500+$ ATIK 16IC mono

Sky-Watcher NWT 150/750 + MII G2-8300

ORION, NWT 500/1500 + MII G2 402

ORION, NWT 500/1500 + MII G2 402

ORION, NWT 500/1500 + MII G2 402

Sky-Watcher NWT 150/750 + MII G2-8300

ORION, NWT 500/1500 + MII G2 402

Sky-Watcher NWT 150/750 + MII G2-8300

NWT 150/600 + Canon 20D + MPCC mk.III

NWT 150/600 + Canon 20D + MPCC mk.III 


\begin{tabular}{|c|c|c|c|c|c|}
\hline \\
\hline HJD-2400000 & Error & $\mathrm{I} / \mathrm{II}$ & Method & Points & Observer \\
\hline 57777.27118 & 0.0001 & II & CCD + Clear & $302 / 89$ & Śmelcer L. \\
\hline 57780.23456 & 0.0001 & I & CCD + Clear & $433 / 43$ & Šmelcer L. \\
\hline 57780.40924 & 0.0001 & II & CCD + Clear & $433 / 253$ & Šmelcer L. \\
\hline 57781.28023 & 0.0001 & $\mathrm{I}$ & CCD + Clear & $317 / 77$ & Šmelcer L. \\
\hline 57781.45494 & 0.0002 & II & CCD + Clear & $317 / 225$ & Šmelcer L. \\
\hline V0405 Gem & & & & & \\
\hline 57777.27017 & 0.0006 & I & CCD + Clear & $283 / 79$ & Šmelcer L. \\
\hline 57780.27939 & 0.0008 & II & CCD + Clear & $362 / 91$ & Šmelcer L. \\
\hline 57780.50426 & 0.0007 & $\mathrm{I}$ & CCD + Clear & $362 / 311$ & Šmelcer L. \\
\hline 57781.41982 & 0.0006 & I & CCD + Clear & $279 / 179$ & Šmelcer L. \\
\hline
\end{tabular}

V0416 Gem 57751.43359 57780.26365

57780.39300

57783.33805

57783.46721

57797.30469

57797.43179

57799.35059

57800.37906

V0428 Gem 57408.34413

V0456 Gem 58154.41805

$\begin{array}{lllll}0.0008 & \text { II } & \text { DSLR } & 65 / 54 & \text { Nosál P. } \\ 0.0006 & \text { I } & \text { DSLR } & 188 / 36 & \text { Nosál P. } \\ 0.0003 & \text { II } & \text { DSLR } & 188 / 128 & \text { Nosál' P. } \\ 0.0006 & \text { I } & \text { DSLR } & 221 / 95 & \text { Nosál' P. } \\ 0.0004 & \text { II } & \text { DSLR } & 221 / 193 & \text { Nosál' P. } \\ 0.0009 & \text { II } & \text { DSLR } & 156 / 38 & \text { Nosál' P. } \\ 0.0011 & \text { I } & \text { DSLR } & 156 / 127 & \text { Nosál' P. } \\ 0.0006 & \text { II } & \text { DSLR } & 202 / 91 & \text { Nosál' P. } \\ 0.0004 & \text { II } & \text { DSLR } & 173 / 89 & \text { Nosál' P. }\end{array}$

0.0002 I CCD + Clear $163 / 65$ Jacobsen J.

EQ6, $80 \mathrm{~mm}$ refractor, $\mathrm{mx} 716$

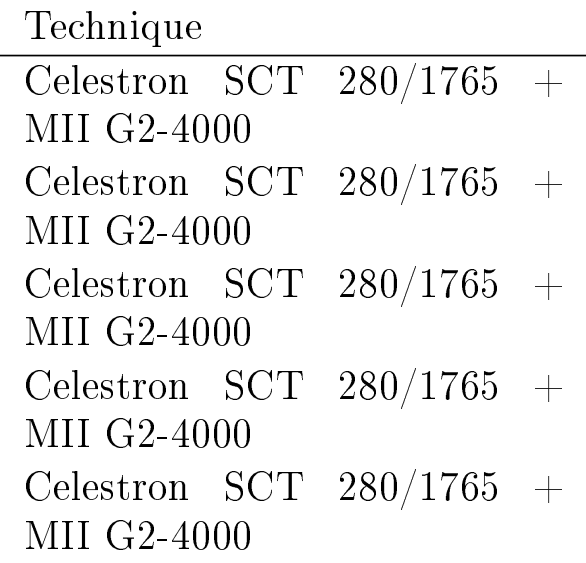

Celestron SCT 280/1765 + MII G2-4000

Celestron SCT 280/1765 + MII G2-4000

Celestron SCT 280/1765 + MII G2-4000

Celestron SCT 280/1765 + MII G2-4000

REF100/500 + Canon 20D

NWT $150 / 600+$ Canon 20D + MPCC mk.III

NWT 150/600 + Canon 20D + MPCC mk.III

NWT 150/600 + Canon 20D + MPCC mk.III

NWT 150/600 + Canon 20D + MPCC mk.III

NWT 150/600 + Canon 20D + MPCC mk.III

NWT $150 / 600+$ Canon 20D + MPCC mk.III

NWT 150/600 + Canon 20D + MPCC mk.III

NWT 150/600 + Canon 20D + MPCC mk.III 


\begin{tabular}{|c|c|c|c|c|c|c|}
\hline $\begin{array}{l}\text { Star } \\
\text { HJD-2400000 }\end{array}$ & Error & $\mathrm{I} / \mathrm{I}]$ & Method & Points & Observer & Technique \\
\hline WW Gem & & & & & & \\
\hline 57839.35396 & 0.0001 & $\mathrm{I}$ & $\mathrm{CCD}+\mathrm{R}$ & $387 / 162$ & Šmelcer L. & $\begin{array}{l}\text { Sky-Watcher NWT 254 } \\
+ \text { MII G2-402 }\end{array}$ \\
\hline YY Gem & & & & & & \\
\hline 57735.48124 & 0.0002 & $\mathrm{I}$ & $\mathrm{CCD}+$ Clear & $724 / 497$ & Červinka L. & $\mathrm{R} 102 / 500+$ Atik 314L + \\
\hline 57737.51762 & 0.0001 & II & $\mathrm{CCD}+$ Clear & $948 / 749$ & Červinka L. & $\mathrm{R} 102 / 500+$ Atik $314 \mathrm{~L}+$ \\
\hline 57737.51768 & 0.0001 & II & CCD + Clear & $945 / 747$ & Červinka L. & $\mathrm{R} 102 / 500+$ Atik 314L + \\
\hline 57739.55179 & 0.0001 & $\mathrm{I}$ & $\mathrm{CCD}+$ Clear & $698 / 611$ & Červinka L. & $\mathrm{R} 102 / 500+$ Atik 314L + \\
\hline
\end{tabular}

AK Her

57887.54350

57909.67107

BC Her

57986.35899

57986.35932

57986.35936
0.0023 II $\quad$ CCD + Clear $53 / 16 \quad$ Hladík B.

0.0001 I Photometer + V50/29 Persha G.
LNS $38 / 135$ + ATIK 320E + MuniWin

Meade RCT 250/2500 + Optec SSP-5a

NWT 355/1600+MII G2-1600, 80/400+G1-0301

NWT 355/1600+MII G2-1600, 80/400+G1-0301

NWT 355/1600+MII G2-1600, 80/400+G1-0301

R102/500 + Atik 314L +
CSS J160915.8+500223 Her

57798.67945
0.0014 I CCD + Clear $511 / 424$ Červinka L.

CSS J173809.9+253941 Her

57892.39594

0.0006 I CCD + Clear $\quad 258 / 59$ Lomoz F.

CzeV522 Her

56489.50266

56502.37470

57214.45633

57242.49681

57989.42907
$0.0002 \mathrm{I} \quad \mathrm{CCD}+\mathrm{R}$

0.0008 II $\mathrm{CCD}+\mathrm{R} \quad 166 / 54 \quad$ Lehky M.

0.0008 II $\quad \mathrm{CCD}+\mathrm{R} \quad 280 / 136$ Lehky M.

0.0007 I $\quad$ CCD $+\mathrm{R} \quad 248 / 228$ Lehky M.

0.0003 I $\quad$ CCD + R 209/168 Lehky M.

277/192 Lehky M.
HK25, NWT 250/1000

SBIG ST-7 + Rc

HK25, NWT 250/1000

SBIG ST-7 + Rc

HK25, NWT 250/1000

SBIG ST-7 + Rc

HK25, NWT 250/1000

SBIG ST-7 + Rc

HK25, NWT 250/1000 SBIG ST-7 + BVRcIc

DK Her 
Star

HJD-2400000

Error I/II Method

Points

Observer

Technique

FN Her

57840.56993

0.0001 I CCD + Clear $\quad 67 / 28 \quad$ Mašek M.

NWT 150/600 + CCD MII G2-1600 + comacorector

IX Her

57968.47345

$0.0005 \mathrm{I} \quad \mathrm{CCD}+\mathrm{R} \quad 55 / 35 \quad$ Lehky $\mathrm{M}$.

JST, NWT 400/2000 + MII

G2-1600 + BVRcIc

57968.47351

0.0008 I $\quad \mathrm{CCD}+\mathrm{I}$

52/32 Lehky M.

JST, NWT 400/2000 + MII

G2-1600 + BVRcIc

57968.47395

$0.0006 \mathrm{I} \quad \mathrm{CCD}+\mathrm{V} \quad 52 / 36 \quad$ Lehky $\mathrm{M}$.

JST, NWT 400/2000 + MII G2-1600 + BVRcIc

Linear 19421081 Her 57978.36436

0.0008 I $\quad$ CCD + Clear 211/59 Bragagnolo U.

Sky-Watcher REF 100/900 + QHY9

MS Her

57928.50745

0.0006 I $\quad$ CCD + R $\quad 181 / 113$ Lehky M.

HK25, NWT 250/1000 + SBIG ST-7 + BVRcIc

ROTSE1 J170250.39+241539.2 Her

$57860.56069 \quad 0.0016$ I DSLR 102/61 Nosál' P.

REF 102/500+ Canon 20D

ROTSE1 J172800.05+380720.6 Her

$57929.57518 \quad 0.0006$ I DSLR

409/233 Školník V.

Tamron LNS 44/300 + Canon $600 \mathrm{D}$

ROTSE1 J173413.59+440118.5 Her

57905.52928 0.0006 I $\quad$ CCD + R $\quad 71 / 54 \quad$ Lehky M.

HK25, NWT 250/1000 + SBIG ST-7 + BVRcIc

ROTSE1 J175527.44+440654.3 Her

57824.66795

0.0006 I $\quad$ CCD + Clear $\quad 477 / 379$ Červinka L.

$\mathrm{R} 102 / 500+$ Atik 314L +

SZ Her

57874.45894

57901.45671

$0 \quad$ I $\quad$ CCD + Clear $277 / 138$ Vrašták M.

NWT $150 / 750+$ G1-0301

0.0001 I DSLR $50 / 30 \quad$ Mašek M.

NWT 130/650 + DSLR Canon $1000 \mathrm{D}+$ comacorector

TX Her

57918.40385

0.0001 I $\quad$ CCD + Clear $\quad 252 / 160$ Červinka L.

Tamron 55-200@135mm, Atik $320 \mathrm{E}$ mono

U Her 
Star

HJD-2400000

Error I/II Method

Points Observer

Technique

UX Her

57902.45026

0.0001 I $\quad$ CCD + R 197/108 Lehky M.

JST, NWT 400/2000 + MII

G2-1600 + BVRcIc

57902.45038

$0 \quad \mathrm{I} \quad \mathrm{CCD}+\mathrm{B}$

173/104 Lehky M.

JST, NWT 400/2000 + MII

G2-1600 + BVRcIc

57902.45044

0.0001 I $\quad$ CCD + V 194/107 Lehky M.

JST, NWT 400/2000 + MII

G2-1600 + BVRcIc

57902.45098

$0.0001 \quad \mathrm{I} \quad \mathrm{CCD}+\mathrm{I}$

195/107 Lehky M.

JST, NWT 400/2000 + MII

G2-1600 + BVRcIc

V0338 Her

57824.58682

0.0001 I CCD + Clear $\quad 642 / 236$ Červinka L.

$\mathrm{R} 102 / 500+$ Atik 314L +

V0477 Her

57846.60231

0.0009 II DSLR

97/69 Nosál' P.

NWT 150/600 + Canon 20D + MPCC mk.III

V0502 Her

57845.54192

0.0001 I CCD + Clear 289/170 Trnka J.

Skywatcher NWT 200/1000 + SBIG ST-9E + comacorre

V0685 Her

57901.42719

0.0003 II $\quad \mathrm{CCD}+\mathrm{R} \quad 165 / 58 \quad$ Lehky M.

JST, NWT 400/2000 + MII

G2-1600 + BVRcIc

V0719 Her

57884.35333

0.0003 I $\quad$ CCD + Clear $\quad 40 / 19 \quad$ Červinka L.

NWT 200/1000 + Atik 314L +

V0819 Her

57924.42334

0.0009 I DSLR

265/81 Školník V.

Tamron LNS 44/300 + Canon 600D

V0829 Her 57825.58203

0.0002 I CCD + Clear $\quad 630 / 209$ Červinka L.

$\mathrm{R} 102 / 500+$ Atik 314L +

V0842 Her

57798.48523

$\begin{array}{lllll}0.0002 & \text { I } & \text { CCD }+ \text { Clear } & 744 / 133 & \text { Červinka L. } \\ 0.0003 & \text { II } & \text { CCD }+ \text { Clear } & 744 / 700 & \text { Červinka L. }\end{array}$

$\mathrm{R} 102 / 500+$ Atik 314L +

57798.69655

$\mathrm{R} 102 / 500+$ Atik 314L +

V0878 Her 57915.64960

0.0004 I Photometer + V50/20 Persha G.

Meade RCT 250/2500 + Optec SSP-5a 
Star

HJD-2400000

V1031 Her 57901.39968

V1033 Her 57978.38919

V1036 Her 57923.47102

57923.47108

57923.47126

V1040 Her 57892.43874

57892.43898

57892.43920

57907.47257

V1041 Her

57876.47057

57876.47080

57876.47114

57876.47131

V1045 Her 57947.44665

V1052 Her 57852.49612
0.0002 I CCD + Clear $\quad 219 / 87$ Urbaník M.

0.0001 I CCD + Clear 213/111 Bragagnolo U.

Sky-Watcher REF 100/900 + QHY9

Sky-Watcher NWT 150/750 + MII G2-8300

HK25, NWT 250/1000 + SBIG ST-7 + BVRcIc

HK25, NWT 250/1000 + SBIG ST-7 + BVRcIc HK25, NWT 250/1000 + SBIG ST-7 + BVRcIc

$\begin{array}{lllcl}0.0002 & \text { II } & \text { CCD }+ \text { I } & 82 / 32 & \text { Lehky M. } \\ 0.0002 & \text { II } & \text { CCD }+ \text { V } & 82 / 38 & \text { Lehky M. } \\ 0.0002 & \text { II } & \text { CCD }+ \text { R } & 82 / 31 & \text { Lehky M. } \\ 0.0002 \text { I } & \text { CCD }+ \text { Clear } & 98 / 43 & \text { Mašek M. }\end{array}$

JST, NWT 400/2000 + MII G2-1600 + BVRcIc

JST, NWT 400/2000 + MII G2-1600 + BVRcIc

JST, NWT 400/2000 + MII G2-1600 + BVRcIc

NWT 150/600 + CCD MII G2-1600 + comacorector

JST, NWT 400/2000 + MII G2-1600 + BVRcIc

JST, NWT 400/2000 + MII G2-1600 + BVRcIc

JST, NWT 400/2000 + MII G2-1600 + BVRcIc

JST, NWT 400/2000 + MII G2-1600 + BVRcIc

Sky-Watcher REF 100/900 + QHY9

NWT $150 / 600+$ Canon 20D + MPCC mk.III 


\begin{tabular}{|c|c|c|c|c|c|}
\hline Star & & & & & \\
\hline HJD-2400000 & Error & I/II Method & Points & Observer & Technique \\
\hline 57847.53507 & 0.0006 & I $\quad$ CCD + Clear & $166 / 73$ & Urbaník M. & $\begin{array}{l}\text { Sky-Watcher NWT 150/750 + } \\
\text { MII G2-8300 }\end{array}$ \\
\hline
\end{tabular}

V1055 Her

57929.44413

57968.39073

V1061 Her 57890.42948

V1062 Her 57905.51487

V1064 Her 57892.42731

V1073 Her 57906.41323

57906.41324

V1097 Her 57868.55380

57868.55384

57868.55406

57973.38111

57973.38189

57973.38254

V1100 Her 57987.33949

\begin{tabular}{|c|c|c|}
\hline 0.0004 II & $\mathrm{CCD}+\mathrm{R}$ & $80 / 51$ \\
\hline $0.0007 \mathrm{I}$ & $\mathrm{CCD}+\mathrm{R}$ & $71 / 42$ \\
\hline
\end{tabular}

0.0001 I CCD+Clear 226/119 Urbaník M.

0.0015 II $\mathrm{CCD}+\mathrm{R} \quad 71 / 45 \quad$ Lehky M.

0.0002 I CCD+Clear 195/156 Urbaník M.

Sky-Watcher NWT 150/750 + MII G2-8300

HK25， NWT 250/1000 + SBIG ST-7 + BVRcIc

Sky-Watcher NWT 150/750 + MII G2-8300

HK25, NWT 250/1000 + SBIG ST-7 + BVRcIc HK25, NWT 250/1000 SBIG ST-7 + BVRcIc

Sky-Watcher NWT 254/1200 + MII G2-402

Sky-Watcher NWT 254/1200 + MII G2-402

\begin{tabular}{|c|c|c|}
\hline $.0001 \mathrm{I}$ & $\mathrm{CCD}+\mathrm{V}$ & $113 / 31$ \\
\hline 0001 & $\mathrm{CCD}+\mathrm{R}$ & $103 / 32$ \\
\hline
\end{tabular}$$
+ \text { MII G2-402 }
$$

HK25, NWT 250/1000 SBIG ST-7 + BVRcIc HK25, NWT 250/1000 + SBIG ST-7 + BVRcIc HK25, NWT 250/1000 SBIG ST-7 + BVRcIC HK25, NWT 250/1000 SBIG ST-7 + BVRcIc HK25， NWT 250/1000 SBIG ST-7 + BVRcIc HK25, NWT 250/1000 SBIG ST-7 + BVRcIc 


\begin{tabular}{llllllll}
\hline $\begin{array}{l}\text { Star } \\
\text { HJD-2400000 }\end{array}$ & Error & I/II Method & Points & Observer & Technique & \\
\hline 58042.32691 & 0.001 & II & CCD + V & $35 / 15$ & Lehky M. & $\begin{array}{l}\text { HK25, NWT 250/1000 } \\
\text { SBIG ST-7 + BVRcIc }\end{array}$ \\
58042.32731 & 0.0005 & II & CCD + I & $38 / 19$ & Lehky M. & $\begin{array}{l}\text { HK25, NWT 250/1000 } \\
\text { HBIG ST-7 + BVRcIc }\end{array}$
\end{tabular}

V1101 Her 57951.46029

V1103 Her 57884.48912

57884.48993

V1105 Her 57906.48651

V1106 Her 57989.33729

V1134 Her 57907.42627

V1148 Her 57873.34629

V1172 Her 57839.60338

V1198 Her 57946.46448

V1207 Her 57827.56785 57908.39381
0.0004 I CCD + Clear 120/103 Bragagnolo U.

Sky-Watcher REF 100/900 QHY9

\begin{tabular}{|c|c|c|c|c|}
\hline $0.0001 \mathrm{II}$ & $\mathrm{CCD}+$ Clear & $236 / 98$ & $\begin{array}{l}\text { Walter F., Bo- } \\
\text { ková S. }\end{array}$ & $\begin{array}{lcc}\text { CZ } & \text { Kometensucher } & \text { REF } \\
200 / 1327+\text { SBIG ST-7 } & \end{array}$ \\
\hline $0.0002 \mathrm{II}$ & DSLR & $219 / 96$ & $\begin{array}{l}\text { Walter F., Bo- } \\
\text { ková S. }\end{array}$ & $\begin{array}{l}\text { CZ Mirotar LNS 180/1000 + } \\
\text { Canon 350d }\end{array}$ \\
\hline
\end{tabular}

0.0006 II CCD + Clear 92/40 Ehrenberger R. Quadruplet LNS 65/420+MII G2-8300

0.0003 I $\quad$ CCD + R $\quad 173 / 58 \quad$ Lehky M.

HK25, NWT 250/1000 + SBIG ST-7 + BVRcIc

0.0004 II CCD+Clear 264/73 Nosál P.

REF $102 / 500+$ ATIK 16IC mono

Meade LX200 f/10 with focal reducer + ST8

0.0001 I CCD+Clear 85/34 Walter F., Hrádek L.

$\mathrm{CZ} \quad$ Kometensucher $200 / 1370+$ SBIG ST7

0.0004 II CCD + Clear $\quad 196 / 71 \quad$ Bragagnolo U. Sky-Watcher REF 100/900 QHY9

NWT $150 / 600+$ Canon 20D + MPCC mk.III

Sky-Watcher NWT 150/750 + MII G2-8300 


\begin{tabular}{|c|c|c|c|c|c|}
\hline Star & & & & & \\
\hline HJD-2400000 & Error & I/II Method & Points & Observer & Technique \\
\hline 57907.53363 & 0.002 & I $\quad$ CCD +Clear & $125 / 119$ & Ehrenberger R. & $\begin{array}{l}\text { Quadruplet LNS } 65 / 420+\mathrm{MII} \\
\text { G2-8300 }\end{array}$ \\
\hline
\end{tabular}

V1215 Her

57874.47300

V1233 Her

57868.37333

57884.36927

57891.35652

57912.47664

V1302 Her 57824.65204

V1309 Her 57824.61432

V1321 Her 57983.47888

V1401 Her 57906.52506

V1408 Her 57890.40887

SY Hor 58155.63950
0.0016 I CCD + Clear $66 / 46 \quad$ Ruocco N.

0.0002 I CCD+Clear 275/140 Urbaník M.

0.0003 I $\quad$ CCD + Clear 231/58 Urbaník M.

0.0002 I CCD+Clear 270/48 Urbaník M.

0.0003 I $\quad$ CCD + Clear $\quad 183 / 111$ Walter F.

0.0004 II CCD + Clear 636/483 Červinka L.

0.0003 I $\quad$ CCD + Clear $\quad 644 / 343$ Červinka L.

0.0004 I CCD + Clear $352 / 187$ Bragagnolo U.

Sky-Watcher REF 100/900 + QHY9

NWT 150/600 + CCD MII G2-1600 + comacorector

Sky-Watcher NWT 150/750 + MII G2-8300

CH Hya 57893.63869

0.0003 II $\quad \mathrm{CCD}+\mathrm{R} \quad 39 / 9 \quad$ Quinones C.

Celestron, SCT, 350/3572

SBIG ST-7 + reducer 0

$\mathrm{H}$. 
Star

HJD-2400000

Error I/II Method

Points Observer

Technique

CSS J081348.6+042637 Hya

57799.53015

0.0003 I CCD + Clear 517/396 Lomoz F.

SNT 254/1016+G2-8300

DF Hya

57811.47303

0.0003 II DSLR

149/109 Hladík B., Walter F.

LNS 62.5/500 + Canon 350d,

57811.47479

0.0001 II CCD + Clear 262/207 Walter F., Hladík B., Malinak 60s, 1600iso J.

$\mathrm{CZ}$ Kometensucher REF $200 / 1237$ + SBIG ST7

CSS J143924.5-263816

Hya

58171.81608

0.0003 I CCD + Clear $147 / 72$ Mašek M.

FRAM, Meade SCT 300/3000 + MII G2-1600

V0474 Hya

57447.30332

0.0002 I CCD + Clear $\quad 193 / 53 \quad$ Jacobsen J.

EQ6, $80 \mathrm{~mm}$ refractor, SXVRH16

WY Hya

58083.81743

$0 \quad$ I $\quad$ CCD + Clear

$279 / 194$

Colazo C., Me- Telescopio STC $16+$ CCD lia R., Starck M. QHY-174

AA Hyi

57727.70885

0.0005 I $\quad$ CCD $+\mathrm{R} \quad 77 / 54 \quad$ Mašek M.

FRAM, Nikkor LNS 106/300 + G4-16000

ASAS J033710-7053.9 Hyi

57746.73450

$0.0007 \mathrm{I} \quad \mathrm{CCD}+\mathrm{R} \quad 87 / 45 \quad$ Mašek M.

FRAM, Nikkor LNS 106/300 + G4-16000

NSV 1174 Hyi

57746.78502

0.0002 II $\mathrm{CCD}+\mathrm{R}$

87/68 Mašek M.

FRAM, Nikkor LNS 106/300

58058.64553

$0.0003 \mathrm{I} \quad \mathrm{CCD}+\mathrm{B} \quad 72 / 34 \quad$ Mašek M.

+ G4-16000

FRAM, Nikkor LNS 106/300

+ G4-16000

NSV 140 Hyi

57727.73446

$0.0002 \mathrm{I} \quad \mathrm{CCD}+\mathrm{R} \quad 77 / 64 \quad$ Mašek M.

FRAM, Nikkor LNS 106/300

+ G4-16000

UV Hyi

57746.74863

0.0002 I $\quad$ CCD + R $\quad 83 / 49 \quad$ Mašek M.

FRAM, Nikkor LNS 106/300 + G4-16000 
Star

HJD-2400000

Error I/II Method

Points

Observer

AR Lac

57991.68516

0.0002 II Photometer + V130/74 Persha G.

Meade RCT 250/2500 + Optec SSP-5a

AW Lac 58085.35239

0.0003 II CCD + Clear 296/191 Bragagnolo U.

Sky-Watcher REF 100/900 + QHY9

CzeV108 Lac

57692.38161

57726.24502

57740.30460

CzeV1251 Lac

58026.32030

0.0012 I $\quad \mathrm{CCD}+\mathrm{V}$

413/251 Červinka L.

218/77 Walter F.

149/34 Walter F.

0.0013 I CCD + Clear $\quad$ 109/48 Walter F

0.002 II DSLR

0.0012 II DSLR

$\mathrm{CCD}+\mathrm{V}$

CzeV758 Lac

57625.44650

57626.56261

CzeV762 Lac

57625.41137

57626.40303

0.0004 II CCD+Clear 227/106 Bílek F.

0.0004 I CCD +Clear 228/183 Bílek F.

0.0005 II CCD + Clear 217/76 Bílek F

0.0006 I CCD + Clear 219/77 Bílek F

ES Lac

57692.30160

0.0017 I DSLR

0.0009 II DSLR

223/38 Walter F.

132/43 Walter F.
Technique
57726.26626

NWT 200/860 + CCD Atik $314 \mathrm{~L}+$

NWT 200/860 + CCD Atik $314 \mathrm{~L}+$

CZ Mirotar SCT 180/1000 + Canon 350d

CZ Mirotar SCT 180/1000 + Canon 350d

Kometensucher Zeiss REF $200 / 1370+$ SBIG ST7

R102/500 + Atik 320E mono, $0.5 \mathrm{x} \mathrm{FC}$

NWT 200/860 + CCD Atik $314 \mathrm{~L}+$

NWT 200/860 + CCD Atik $314 \mathrm{~L}+$

CZ Mirotar SCT 180/1000 Canon 350d

CZ Mirotar SCT 180/1000 +

HBHA 4705-03 Lac 57625.31461

0.0001 I CCD + Clear 227/16 Bílek F.

0.0002 II CCD + Clear 227/134 Bílek F Canon 350d

NWT 200/860 + CCD Atik $314 \mathrm{~L}+$

NWT 200/860 + CCD Atik $314 \mathrm{~L}+$ 


\begin{tabular}{|c|c|c|c|c|c|}
\hline \multicolumn{6}{|l|}{ Star } \\
\hline HJD-2400000 & Error & I/II Method & Points & Observer & Technique \\
\hline 57626.34664 & 0.0003 & I $\quad$ CCD + Clear & $218 / 36$ & Bílek F. & $\begin{array}{l}\text { NWT 200/860 + CCD Atik } \\
314 \mathrm{~L}+\end{array}$ \\
\hline 57626.51779 & 0.0002 & $\mathrm{CCD}+$ Clear & $218 / 149$ & Bílek F. & $\begin{array}{l}\text { NWT } 200 / 860+\text { CCD Atik } \\
314 \mathrm{~L}+\end{array}$ \\
\hline
\end{tabular}

IP Lac 57993.53656

LY Lac 57906.45096 57923.44807

LZ Lac 57907.47935

SvkV014 Lac 57996.41323

SW Lac 57958.49868

57958.49869

TW Lac 57763.30758

V0364 Lac 57714.39608 57714.39712 V0430 Lac 58027.48073 58027.48124 58044.52629
0.0004 II CCD + Clear $69 / 22$ Červinka L.

R102/500 + Atik 320E mono, $0.5 \mathrm{x} F \mathrm{C}$

$\begin{array}{lllll}0.0002 \text { I } & \text { CCD }+ \text { Clear } & 146 / 82 & \begin{array}{l}\text { Mašek M., Tyl- } \\ \text { šar M. }\end{array} & \begin{array}{l}\text { NWT 250/1000 + CCD MII } \\ \text { G2-8300 }\end{array} \\ 0.0001 \text { I } & \text { CCD }+ \text { Clear } & 143 / 84 & \begin{array}{l}\text { Mašek M., Tyl- } \\ \text { NWT 250/1000 + CCD MII } \\ \text { Sar M2-8300 }\end{array}\end{array}$

0.0003 II CCD+Clear 147/104 Mašek M., Tylšar $M$.

NWT 250/1000 + CCD MII G2-8300

$0.0001 \mathrm{I} \quad \mathrm{CCD}+\mathrm{R} \quad 120 / 54 \quad$ Vrašták M

NWT 355/1600+MII G2-1600, $80 / 400+$ G1-0301

Sky-Watcher NWT 254/1200 + MII G2-402

Sky-Watcher NWT 254/1200 + MII G2-402

Celestron SCT 280/1765 + MII G2-4000

Celestron SCT $355 / 2460+$ MII G2-1600

Celestron SCT 355/2460 + MII G2-1600

JST, NWT 400/2000 + MII G2-1600 + BVRcIc JST, NWT 400/2000 + MII G2-1600 + BVRcIc JST, NWT 400/2000 + MII G2-1600 + BVRcIc 


\begin{tabular}{|c|c|c|c|c|c|}
\hline $\begin{array}{l}\text { Star } \\
\text { HJD-2400000 }\end{array}$ & Error & $\mathrm{I} / 1$ & Method & Points & Observer \\
\hline $\begin{array}{l}\text { V0441 Lac } \\
57923.43811\end{array}$ & 0.0009 & II & CCD + Clear & $196 / 80$ & Červinka I \\
\hline $\begin{array}{l}\text { V0492 Lac } \\
58027.31337\end{array}$ & 0.0004 & II & $\mathrm{CCD}+\mathrm{R}$ & $149 / 27$ & Lehky M. \\
\hline 58027.31393 & 0.0004 & II & $\mathrm{CCD}+\mathrm{I}$ & $124 / 25$ & Lehky M. \\
\hline 58027.44249 & 0.0004 & $\mathrm{I}$ & $\mathrm{CCD}+\mathrm{I}$ & $124 / 77$ & Lehky M. \\
\hline 58027.44264 & 0.0004 & $\mathrm{I}$ & $\mathrm{CCD}+\mathrm{R}$ & $149 / 79$ & Lehky M. \\
\hline 58027.57074 & 0.0006 & II & $\mathrm{CCD}+\mathrm{I}$ & $124 / 119$ & Lehky M. \\
\hline 58027.57148 & 0.0004 & II & $\mathrm{CCD}+\mathrm{R}$ & $149 / 135$ & Lehky M. \\
\hline 58042.25731 & 0.0006 & II & $\mathrm{CCD}+\mathrm{V}$ & $130 / 9$ & Lehky M. \\
\hline 58042.38607 & 0.0005 & $\mathrm{I}$ & $\mathrm{CCD}+\mathrm{V}$ & $130 / 73$ & Lehky M. \\
\hline 58043.28700 & 0.0005 & II & $\mathrm{CCD}+\mathrm{V}$ & $148 / 19$ & Lehky M. \\
\hline 58043.41541 & 0.0009 & $\mathrm{I}$ & $\mathrm{CCD}+\mathrm{V}$ & $148 / 94$ & Lehky M. \\
\hline 58044.31846 & 0.0005 & II & $\mathrm{CCD}+\mathrm{V}$ & $195 / 29$ & Lehky M. \\
\hline 58044.44616 & 0.0006 & $\mathrm{I}$ & $\mathrm{CCD}+\mathrm{V}$ & $195 / 114$ & Lehky M. \\
\hline 58095.32869 & 0.0004 & II & $\mathrm{CCD}+\mathrm{V}$ & $159 / 87$ & Lehky M. \\
\hline
\end{tabular}

V0505 Lac 58026.25686

VX Lac 57645.31880

AL Leo 57800.49401 58080.65671
$0.0006 \mathrm{I} \quad \mathrm{CCD}+\mathrm{V} \quad 501 / 54 \quad$ Červinka L.

R102/500 + Atik 320E mono, $0.5 \mathrm{x}$ FC

JST, NWT 400/2000 + MII G2-1600 + BVRcIc

JST, NWT 400/2000 + MII G2-1600 + BVRcIc

JST, NWT 400/2000 + MII G2-1600 + BVRcIc

JST, NWT 400/2000 + MII G2-1600 + BVRcIc

JST, NWT 400/2000 + MII

G2-1600 + BVRcIc

JST, NWT $400 / 2000+$ MII G2-1600 + BVRcIc

JST, NWT 400/2000 + MII

G2-1600 + BVRcIc

JST, NWT 400/2000 + MII G2-1600 + BVRcIc

JST, NWT 400/2000 + MII G2-1600 + BVRcIc

JST, NWT 400/2000 + MII G2-1600 + BVRcIc

JST, NWT 400/2000 + MII G2-1600 + BVRcIc

JST, NWT 400/2000 + MII G2-1600 + BVRcIc

JST, NWT 400/2000 + MII G2-1600 + BVRcIc

0.0001 I $\quad$ CCD + Clear $\quad 183 / 99 \quad$ Červinka L. $\quad$ R102/500 + Atik 314L + 


\begin{tabular}{|c|c|c|c|c|c|}
\hline $\begin{array}{l}\text { Star } \\
\text { HJD-2400000 }\end{array}$ & Error & I/II Method & Points & Observer & Technique \\
\hline AM Leo & & & & & \\
\hline 58112.58577 & 0.0002 & $\mathrm{CCD}+\mathrm{V}$ & $228 / 123$ & Magris M. & $\begin{array}{l}\text { Achromatic refractor 120/600, } \\
\text { CCD Orion Starshoo }\end{array}$ \\
\hline
\end{tabular}

AP Leo

57829.53803

58199.43877
0.0002 II DSLR

0.0001 I $\quad C C D+$ Clear
203/111 Hladík B., Walter $\mathrm{F}$

225/179 Urbaník M.
TamronSP LNS 62.5/500 + Canon 350d, 50s, 1600is Sky-Watcher MAK 102/1300 + MII G2-8300

ASAS $1110916+0223.3$ Leo 57829.47702 0.0002 I

$\mathrm{CCD}+$ Clear

291/125 Walter F., Hla-

$\mathrm{CZ}$ Kometensucher dík B., Novotný P. 200/1237 + SBIG ST7

ASAS J103034+2516.9 Leo 58163.33375

0.0003 II $\mathrm{CCD}+\mathrm{R}$ 0.0002 I $\quad \mathrm{CCD}+\mathrm{R}$ 0.0002 I $\mathrm{CCD}+$ Clear

58175.35518

ASAS J103929+1641.8 Leo 57839.54169 0.0006 I DSLR

776/558 Školník V.

677/116 Lomoz F.

SNT254/1016+G2-8300

SNT254/1016+G2-8300

SNT254/1016+G2-8300

Tamron LNS 44/300 + Canon 600D

BZ Leo

57844.43727

0.0002 I $\quad C C D+$ Clear

488/187 Lomoz F.

STN 254/1016+G2-8300

57844.57147

0.0002 II $\mathrm{CCD}+$ Clear

488/387 Lomoz F.

SNT 254/1016+G2-8300

CE Leo

57772.62308

0.0002 I DSLR

109/47 Nosál' P.

NWT 150/600 + Canon 20D + MPCC mk.III

57844.38412

0.0001 II $\mathrm{CCD}+$ Clear

$110 / 44$

Mašek M., Tylšar M.

NWT 250/1000 + CCD MII

57845.44574

$0.0001 \mathrm{I} \quad \mathrm{CCD}+$ Clear

216/156 Mašek M., Tylšar M.

NWT 250/1000 + CCD MII G2-8300

CSS J114242.5+233229 Leo

57844.38907

57845.46263

CzeV370 Leo

57841.38208

58171.58809
0.0002 I $\quad$ CCD + Clear $\quad 110 / 50$

0.0003 I $\mathrm{CCD}+$ Clear
Mašek M., Tylšar $M$.

214/168 Mašek M., Tylšar M.
NWT 250/1000 + CCD MII G2-8300

NWT 250/1000 + CCD MII G2-8300
0.0003 II CCD + Clear $184 / 69$ Mašek M.

0.0005 II CCD + Clear 70/43 Mašek M.
NWT 150/600 + CCD MII G2-1600 + comacorector NWT 150/600 + CCD MII G2-1600 + comacorector 
Star

HJD-2400000

Error I/II Method

Points Observer

Technique

DO Leo

57853.50745

0.0011 I CCD + Clear 314/248 Lomoz F.

SNT 254/1016+G2-8300

ET Leo

57840.32324

$0.002 \quad \mathrm{I} \quad \mathrm{CCD}+$ Clear

82/22 Hladík B.

+ ATIK 320E + MuniWin

0.0007 I $\quad$ CCD $+\mathrm{V} \quad$ 254/123 López O.

NWT 203/1000 + Webcam $(\mathrm{ICX} 098 \mathrm{AK})+$ Baader IR $/ \mathrm{Cu}$

\section{EX Leo}

57839.47542

0.001 I DSLR

839/396 ک̌̉kolník V.

Tamron LNS 44/300 + Canon $600 \mathrm{D}$

FM Leo

58181.49659

$0.0003 \mathrm{I} \quad \mathrm{CCD}+\mathrm{R} \quad 82 / 39 \quad$ Mašek M.

NWT 150/600 + CCD MII

G2-1600 + comacorector

FS Leo

57781.57144

0.0007 II CCD + Clear $\quad 95 / 38 \quad$ Mašek M.

NWT $150 / 600+$ CCD MII

58177.53276

0.0003 I CCD + Clear $70 / 48 \quad$ Mašek M.

G2-1600 + comacorector

NWT 150/600 + CCD MII G2-1600 + comacorector

GSC 00853-00371 Leo

57829.36933

0.0005 II $\quad \mathrm{CCD}+$ Clear $22 / 4$

Salvaggio

F., $\quad$ C9.25 + ST8-XME

Banfi

M.,

Papini R.,

GV Leo

57840.31944

0.0001 II $\mathrm{CCD}+\mathrm{R}$

87/12 Lehky M.

57840.31965

0.0002 II $\mathrm{CCD}+\mathrm{B}$

83/12 Lehky M.

57840.31967

0.0001 II $\mathrm{CCD}+\mathrm{V}$

85/12 Lehky M.

57840.31977

0.0002 II $\mathrm{CCD}+\mathrm{I}$

86/12 Lehky M.

57840.45286

$0.0002 \mathrm{I} \quad \mathrm{CCD}+\mathrm{R} \quad 87 / 60 \quad$ Lehky $\mathrm{M}$.

57840.45293

0.0002 I $\quad \mathrm{CCD}+\mathrm{I}$

86/58 Lehky M.

57840.45330

0.0002 I $\quad \mathrm{CCD}+\mathrm{V}$

85/57 Lehky M.

57840.45333

0.0003 I $\quad \mathrm{CCD}+\mathrm{B}$

83/55 Lehky M.

JST, NWT 400/2000 + MII

G2-1600 + BVRcIc

JST, NWT 400/2000 + MII

G2-1600 + BVRcIc

JST, NWT 400/2000 + MII

G2-1600 + BVRcIc

JST, NWT 400/2000 + MII

G2-1600 + BVRcIc

JST, NWT 400/2000 + MII

G2-1600 + BVRcIc

JST, NWT 400/2000 + MII

G2-1600 + BVRcIc

JST, NWT 400/2000 + MII

G2-1600 + BVRcIc

JST, NWT 400/2000 + MII

G2-1600 + BVRcIc

NWT 150/600 + CCD MII G2-1600 + comacorector 


\begin{tabular}{|c|c|c|c|c|c|}
\hline Star & & & & & \\
\hline HJD-2400000 & Error & I/II Method & Points & Observer & Technique \\
\hline 57844.45402 & 0.0001 & I $\quad$ CCD + Clear & $89 / 74$ & Mašek M. & $\begin{array}{l}\text { NWT } 150 / 600+\text { CCD MII } \\
\text { G2-1600 + comacorector }\end{array}$ \\
\hline
\end{tabular}

GW Leo

$\begin{array}{lllllll}57798.54062 & 0.0012 & \text { II } & \text { CCD }+ \text { R } & 72 / 26 & \text { Lehky M. } & \begin{array}{l}\text { JST, NWT } 400 / 2000+\text { MII } \\ \text { G2- } 1600+\text { BVRcIc }\end{array} \\ 57798.54170 & 0.001 & \text { II } & \text { CCD }+ \text { V } & 73 / 26 & \text { Lehky M. } & \begin{array}{l}\text { JST, NWT } 400 / 2000+\text { MII } \\ \text { G2-1600 + BVRcIc }\end{array}\end{array}$

HI Leo

57798.56162

0.0001 II CCD + Clear 87/56 Mašek M.

NWT 150/600 + CCD MII G2-1600 + comacorector

HS Leo

57853.40352

57853.57465

0.0002 I $\quad$ CCD + Clear $\quad 78 / 28 \quad$ Banfi M.

Celestron $8+$ Moravian 8300

0.0006 II $\mathrm{CCD}+$ Clear $78 / 74 \quad$ Banfi $\mathrm{M}$.

Celestron $8+$ Moravian 8300

LINEAR 1438520 Leo 58199.35835

$\begin{array}{rlrl}0.0006 \text { I CCD }+ \text { Clear 78/15 } & \text { Salvaggio F., } 12+\text { STX6303 } \\ & \text { Papini } & \text { Marchini A. }\end{array}$

MP Leo

57844.40914

0.0002 I CCD + Clear 85/51 Mašek M.

NWT 150/600 + CCD MII G2-1600 + comacorector

NSVS 7541088 Leo

58175.34357

0.0002 I CCD + Clear $752 / 256$ Lomoz F.

SNT254/1016+G2-8300

NT Leo

57776.67913

$0.0004 \mathrm{I} \quad \mathrm{CCD}+\mathrm{R} \quad 100 / 63 \quad$ Mašek M.

NWT 150/600 + CCD MII G2-1600 + comacorector

UCAC4 532-048414

Leo

58176.42790

0.004 I $\quad C C D+$ Clear 85/19 Mašek M.

NWT 150/600 + CCD MII

G2-1600 + comacorector

UX Leo

58185.74731

0.0011 II $\quad \mathrm{CCD}+\mathrm{R} \quad 220 / 29 \quad$ Mašek M.

FRAM, Meade SCT 300/3000

+ MII G2-1600

VZ Leo

58176.49822

0.0002 I CCD + Clear 84/50 Mašek M.

NWT 150/600 + CCD MII 


\begin{tabular}{|c|c|c|c|c|c|}
\hline $\begin{array}{l}\text { Star } \\
\text { HJD-2400000 }\end{array}$ & Error & I/II Method & Points & Observer & Technique \\
\hline WY Leo & & & & & \\
\hline 57839.34552 & 0.0011 & CCD + Clear & $236 / 74$ & Urbaník M. & $\begin{array}{l}\text { Sky-Watcher NWT 150/750 + } \\
\text { MII G2-8300 }\end{array}$ \\
\hline 57839.34740 & 0.0008 & $\mathrm{CCD}+$ Clear & $295 / 54$ & Trnka J. & $\begin{array}{l}\text { Skywatcher NWT 200/1000 + } \\
\text { SBIG ST-9E + comacorre }\end{array}$ \\
\hline $\begin{array}{l}\text { XY Leo } \\
57706.59258\end{array}$ & - & Clopo & & 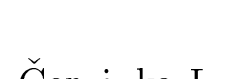 & D102/500 \\
\hline
\end{tabular}

1SWASP J060334.68-212558.6 Lep

57722.69377

0.0013

$\mathrm{CCD}+\mathrm{R} \quad 100 / 69 \quad$ Mašek M.

0.0006 I $\quad \mathrm{CCD}+\mathrm{R} \quad 96 / 54 \quad$ Mašek M.

FRAM, Nikkor LNS 106/300 + G4-16000

ASAS J060247-2227.7 Lep

57722.66479

0.0006 I $\quad$ CCD + R $\quad 88 / 25 \quad$ Mašek M.

FRAM, Nikkor LNS 106/300

+ G4-16000

RS Lep

57722.68153

$0.0001 \quad \mathrm{I} \quad \mathrm{CCD}+\mathrm{R} \quad 92 / 63 \quad$ Mašek $\mathrm{M}$.

Lep

FRAM, Nikkor LNS 106/300 + G4-16000

CSS J055444
57722.61132

FRAM, Nikkor LNS 106/300

+ G4-16000

AG LMi

57407.33576

0.0002 I CCD +Clear 270/115 Jacobsen J.

EQ6, $100 \mathrm{~mm}$ refractor, Atik 314

57716.51082

0.0001 I CCD +Clear 105/65 Medulka T.

REF 90/500 + MII G1-300

57814.36081

58200.32111

0.0001 I $\quad \mathrm{CCD}+$ Clear

156/74 Ehrenberger R.

0.0001 I $\quad C C D+$ Clear 211/99 Urbaník M.

RT LMi

57739.68386

0.0005 II CCD + Clear $\quad 152 / 71$ Bragagnolo U.

Sky-Watcher REF 100/900 + QHY9
Tamron LNS 44/300 + Canon 600D 


\begin{tabular}{|c|c|c|c|c|c|}
\hline $\begin{array}{l}\text { Star } \\
\text { HJD-2400000 }\end{array}$ & Error & I/II Method & Points & Observer & Technique \\
\hline WZ LMi & & & & & \\
\hline 58166.40429 & 0.0004 & $\mathrm{CCD}+\mathrm{V}$ & $75 / 44$ & Vrašták M & NWT 355/1600+MII G2-1600 \\
\hline 58166.40489 & 0.0003 & $\mathrm{CCD}+\mathrm{R}$ & $81 / 47$ & Vrašták M & NWT 355/1600+MII G2-1600 \\
\hline $\begin{array}{l}\text { XY LMi } \\
58118.50021\end{array}$ & 0.0006 & $\mathrm{CCD}+\mathrm{V}$ & $110 / 51$ & Magris M. & $\begin{array}{l}\text { REF } 120 / 600+\text { Orion } 3 \mathrm{G} \\
\mathrm{CCD}+0.5 \mathrm{x} \text { reducer }\end{array}$ \\
\hline
\end{tabular}

OS Lup

57892.68934
$0 \quad$ I $\quad$ CCD + Clear 757/399 Girardini C., Meade LX200 SC + CCD
Colazo
C.,
Starck M.

SX Lup

58187.75929

0.0001 I $\quad C C D+$ Clear $144 / 57$ Mašek M.

FRAM, Meade SCT 300/3000

+ MII G2-1600

2MASS J06261950+2936271 Lyn

58178.33787

0.0006 I CCD + Clear 168/86 Lomoz F.

SNT254/1016+G2-8300

2MASS J06320975+2954573 Lyn

58178.31191

0.0004 I CCD + Clear 231/82 Lomoz F.

NWT 300/1200+ST2000XM

BH Lyn

57715.50162

0.0001 I CCD + Clear 203/82 Šmelcer L.

Celestron SCT 280/1765 + MII G2-4000

57853.45020

$0.0003 \mathrm{I} \quad \mathrm{CCD}+\mathrm{R} \quad$ 169/97 Hanžl D.

DATEL, NWT 200/800 + MII G2 8300

58186.55645

$0.0002 \mathrm{I} \quad \mathrm{CCD}+\mathrm{R} \quad 92 / 37 \quad$ Šmelcer $\mathrm{L}$.

Celestron SCT 355/2460 + MII G2-1600

CC Lyn

57788.54734

0.0008 II Photometer + V43/23 Persha G.

Meade RCT 250/2500 + Optec SSP-5a

CF Lyn

58181.47643

0.0003 I CCD+Clear 297/157 Urbaník M.

Sky-Watcher NWT 80/520 MII G2-8300

CS Lyn 
Star

HJD-2400000

CzeV242 Lyn

57828.38423

57828.53235

EL Lyn

57828.44283

$0.0001 \mathrm{I} \quad \mathrm{CCD}+\mathrm{R}$

359/205 ̌melcer L.

$0.0003 \mathrm{I} \quad \mathrm{CCD}+\mathrm{R} \quad 307 / 132 \quad$ Šmelcer L.

0.0006 II $\mathrm{CCD}+\mathrm{R} \quad 307 / 272$ Šmelcer L.

$0.0004 \mathrm{I} \quad \mathrm{CCD}+\mathrm{R}$

300/166 Hanžl D.

57735.53966

FO Lyn

57782.54380

FP Lyn

57775.62850

NSVS 4732433 Lyn

58113.41326

NSVS 4751449 Lyn

57752.43603

$\begin{array}{llll}0.0002 \text { I } \quad \text { CCD }+ \text { Clear } 170 / 65 & \begin{array}{l}\text { Mašek M } \\ \text { šar M. }\end{array} \\ 0.0002 \text { I } \quad \text { CCD }+ \text { Clear } & 232 / 133 \text { Trnka J. }\end{array}$

0.0002 I DSLR $\quad 139 / 36 \quad$ Walter F.

0.0003 I CCD + Clear 227/149 Walter F

Set sekce, NWT 150/750 + G2-1600

DATEL, NWT 200/800 + MII

G2 8300

Celestron SCT 355/2460

MII G2-1600

Celestron SCT 355/2460 +

MII G2-1600

Celestron SCT 355/2460 + MII G2-1600

NWT 150/750, Canon 450 D

RV Lyn

58106.41413

58106.41431

RZ Lyn

57800.45426

0.0002 I CCD + Clear 95/49 Lehky M.

$\begin{array}{lllll}0.0001 & \text { I } & \text { CCD }+\mathrm{V} & 95 / 59 & \text { Vrašták M } \\ 0.0001 & \text { I } & \text { CCD }+\mathrm{R} & 92 / 57 & \text { Vrašták M }\end{array}$

SX Lyn

57752.25904

0.0003 I CCD + Clear $\quad 150 / 81$ Červinka L.

UU Lyn 57774.53357

58149.29443
0.0001 I CCD + Clear $\quad 139 / 47$ Bragagnolo U.

0.0002 I $\quad$ CCD + R $\quad 105 / 51 \quad$ Hanžl D.
NWT 355/1600+MII G2-1600

NWT 355/1600+MII G2-1600

$\mathrm{R} 102 / 500+$ Atik 314L +

Skywatcher NWT 200/1000+ SBIG ST-9E + comacorre

HK25, NWT 250/1000 + SBIG ST-7 + C

Sky-Watcher REF 100/900 + QHY9

DATEL, NWT 200/800 + MII G2 8300 
Star

HJD-2400000

TZ Lyr

57887.42008

DT Lyr

57920.46478

DU Lyr

57920.39555

FL Lyr

57929.49090

IP Lyr

57721.23812

57721.23883

57841.56803

57841.56858

57924.54345

57924.54376

57924.54411

KIC 2972514 Lyr

57906.50151

57907.50857

57907.50875
0.0106 II $\mathrm{CCD}+$ Clear $71 / 18$ Ruocco N.

0.0004 I CCD + Clear 91/63 Urbaník M.

0.0003 I CCD + Clear 111/19 Urbaník M.

0.0002 I DSLR

63/38 Mašek M.

Zeiss Sonnar LNS 64/180+ DSLR Canon 1000D
Meade LX200 f/10 with focal reducer $+\mathrm{ST} 8$

Sky-Watcher NWT 150/750 + MII G2-8300

Sky-Watcher NWT 150/750 + MII G2-8300

$\begin{array}{lllll}0.0005 & \text { I } & \text { CCD }+\mathrm{V} & 29 / 20 & \text { Lehky M. } \\ 0.0003 \text { I } & \text { CCD }+ \text { R } & 33 / 24 & \text { Lehky M. } \\ 0.0004 \text { II } & \text { CCD }+\mathrm{V} & 55 / 18 & \text { Lehky M. } \\ 0.0004 \text { II } & \text { CCD }+ \text { R } & 59 / 23 & \text { Lehky M. } \\ 0.0003 \text { I } & \text { CCD }+ \text { I } & 26 / 14 & \text { Lehky M. } \\ 0.0002 \text { I } & \text { CCD }+ \text { R } & 25 / 14 & \text { Lehky M. } \\ 0.0002 \text { I } & \text { CCD }+\mathrm{V} & 26 / 13 & \text { Lehky M. }\end{array}$

JST, NWT 400/2000 + MII

G2-1600 + BVRcIc

JST, NWT 400/2000 + MII

G2-1600 + BVRcIc

JST, NWT $400 / 2000+$ MII

G2-1600 + BVRcIc

JST, NWT 400/2000 + MII

G2-1600 + BVRcIc

JST, NWT $400 / 2000+$ MII

G2-1600 + BVRcIc

JST, NWT 400/2000 + MII

G2-1600 + BVRcIc

JST, NWT 400/2000 + MII

G2-1600 + BVRcIc

NWT 250/1000 + ATIK 314L $+\mathrm{MPCC}$

MNT $\quad 190 / 1000$

ATIK414EXM

NWT 250/1000 + ATIK 314L

$+\mathrm{MPCC}$

KID 02694741 Lyr

57907.48110

0.0003 I $\quad$ CCD + Clear 222/109 Lomoz F.

SNT 254/1016+G2-8300 


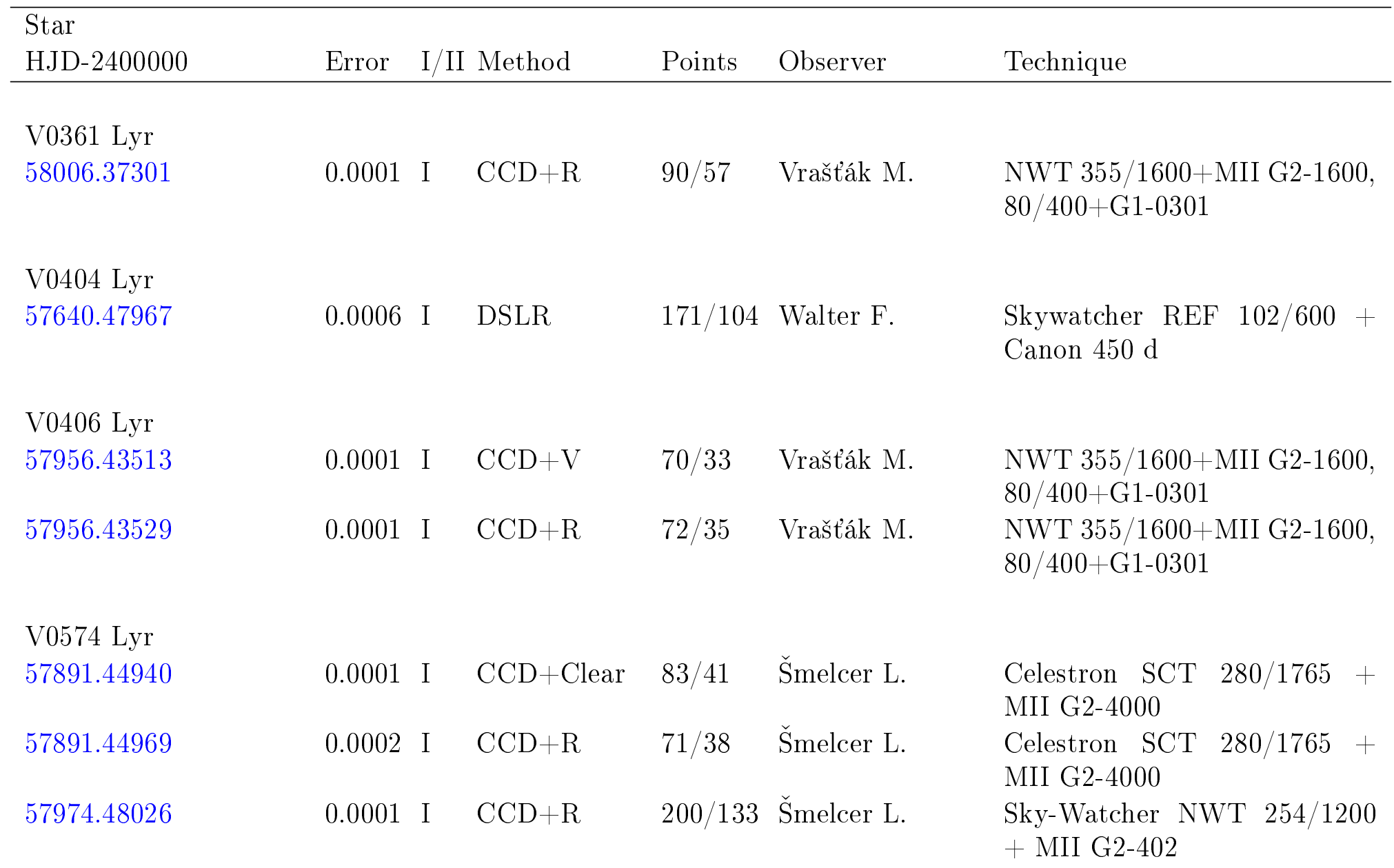

V0576 Lyr 57996.32866

V0589 Lyr 57700.28502

V0648 Lyr 57879.46440
0.0004 I CCD + Clear 150/64 Bragagnolo U.

Sky-Watcher REF 100/900 + QHY9

0.0004 I CCD +Clear 165/101 Bragagnolo U. Sky-Watcher REF 100/900 + QHY9

0.0019 II $\quad$ CCD + Clear $\quad 64 / 23 \quad$ Ruocco N.

Meade LX200 f/10 with focal reducer + ST8

ASAS J044516-7060.0 Men

58060.63110

0.0015 I $\mathrm{CCD}+\mathrm{B} \quad 77 / 33$ Mašek M.
FRAM, Nikkor LNS 106/300 + G4-16000 


\begin{tabular}{|c|c|c|c|c|c|}
\hline $\begin{array}{l}\text { Star } \\
\text { HJD-2400000 }\end{array}$ & Error & I/II Method & Points & Observer & Technique \\
\hline FW Mon & & & & & \\
\hline 57435.34155 & 0.0005 & $\mathrm{CCD}+$ Clear & $207 / 107$ & Jacobsen J. & EQ6, $80 \mathrm{~mm}$ refractor, $\mathrm{mx} 716$ \\
\hline GDS J065320 & Mon & & & & \\
\hline 57800.33237 & 0.0022 & $\mathrm{CCD}+$ Clear & $101 / 65$ & Mašek M. & $\begin{array}{l}\text { NWT } 150 / 600+\text { CCD MII } \\
\text { G2-1600 + comacorector }\end{array}$ \\
\hline
\end{tabular}

GDS J0657299-002350 Mon

$57800.27819 \quad 0.0004$ I $\quad$ CCD + Clear $100 / 31$ Mašek M.

NWT 150/600 + CCD MII G2-1600 + comacorector

GH Mon

57841.31837

0.0001 I $\quad$ CCD + Clear $\quad 84 / 49 \quad$ Trnka J.

Skywatcher NWT 200/1000+ SBIG ST-9E + comacorre

MX Mon

58126.76036

0.0014 I CCD + Clear 50/21 Mašek M.

FRAM, Nikkor LNS 106/300

+ G4-16000

NN Mon

58080.54666

0.0001 I CCD + Clear 57/24 Mašek M.

NWT 150/600 + CCD MII G2-1600

58155.36001

0.0001 I CCD + Clear 118/48 Mašek M.

NWT 150/600 + CCD MII

58171.32717

0.0008 II $\quad$ CD + Clear 106/37 Mašek M.

G2-1600 + comacorector

NWT 150/600 + CCD MII G2-1600 + comacorector

NS Mon

57764.33723

0.0003 I CCD+Clear 242/95 Urbaník M.

Sky-Watcher REF 80/520 + MII G2-8300

V0521 Mon 57800.30250

0.0003 II CCD +Clear 102/46 Mašek M.

NWT $150 / 600+$ CCD MII G2-1600 + comacorector

V0753 Mon 58126.76410

0.0005 II CCD +Clear 75/26 Mašek M.

FRAM, Nikkor LNS 106/300 + G4-16000

V0864 Mon 58126.76279

0.0004 II CCD +Clear 74/24 Mašek M.

FRAM, Nikkor LNS 106/300 + G4-16000 


\begin{tabular}{|c|c|c|c|c|c|c|}
\hline $\begin{array}{l}\text { Star } \\
\text { HJD-2400000 }\end{array}$ & Error & $\mathrm{I} / \mathrm{I}$ & Method & Points & Observer & Technique \\
\hline V0936 Mon & & & & & & \\
\hline 58155.33415 & 0.0005 & I & CCD + Clear & $92 / 36$ & Mašek M. & $\begin{array}{l}\text { NWT } 150 / 600+\text { CCD MII } \\
\text { G2-1600 + comacorector }\end{array}$ \\
\hline 58171.38293 & 0.0005 & II & $\mathrm{CCD}+$ Clear & $106 / 63$ & Mašek M. & $\begin{array}{l}\text { NWT } 150 / 600+\text { CCD MII } \\
\text { G2-1600 + comacorector }\end{array}$ \\
\hline V0958 Mon & & & & & & \\
\hline 57725.56237 & 0.0005 & I & DSLR & $324 / 167$ & Walter F. & $\begin{array}{l}\text { Tamron SP LNS } 62.5 / 500+ \\
\text { Canon 350d }\end{array}$ \\
\hline 57749.41839 & 0.0007 & I & DSLR & $196 / 68$ & Walter F. & $\begin{array}{l}\text { Tamron SP LNS } 62.5 / 500+ \\
\text { Canon 350d }\end{array}$ \\
\hline 57749.56759 & 0.0014 & II & DSLR & $196 / 166$ & Walter F. & $\begin{array}{l}\text { Tamron SP LNS } 62.5 / 500+ \\
\text { Canon 350d }\end{array}$ \\
\hline V0984 Mon & & & & & & \\
\hline 57715.76041 & 0.0003 & II & $\mathrm{CCD}+\mathrm{R}$ & $157 / 111$ & Mašek M. & $\begin{array}{l}\text { FRAM, Nikkor LNS } 106 / 300 \\
\text { + G4- } 16000\end{array}$ \\
\hline
\end{tabular}

LL Mus 57743.78419 $0.0004 \mathrm{I} \quad \mathrm{CCD}+\mathrm{R} \quad 53 / 31 \quad$ Mašek M.

FRAM, Nikkor LNS 106/300 + G4-16000

V0501 Oph 57923.49358 57924.46144 57926.39668 V0590 Oph 57931.45498 57938.49044 57948.40949 57960.39846

\begin{tabular}{|c|c|c|c|}
\hline 0002 & II & $\mathrm{CCD}+$ Clear & $91 / 47$ \\
\hline 00 & II & CCD + Clear & $129 / 65$ \\
\hline & & $\mathrm{CCD}+$ Clear & $97 / 24$ \\
\hline
\end{tabular}

138/72 Nosál P.

127/88 Nosál P.

123/43 Nosál P.

96/39 Nosál' P.
NWT 150/600 + CCD MII G2-1600 + comacorector NWT 150/600 + CCD MII G2-1600 + comacorector NWT 150/600 + CCD MII G2-1600 + comacorector
NWT 150/600 + Canon 20D + MPCC mk.III

NWT 150/600 + Canon 20D + MPCC mk.III

NWT 150/600 + Canon 20D + MPCC mk.III

NWT 150/600 + Canon 20D + MPCC mk.III 


\begin{tabular}{|c|c|c|c|c|c|c|}
\hline Star & & & & & & \\
\hline HJD-2400000 & Error & $\mathrm{I} / \mathrm{Il}$ & Method & Points & Observer & Technique \\
\hline 57917.52254 & 0.0003 & II & $\mathrm{CCD}+\mathrm{B}$ & $68 / 49$ & Lehky M. & $\begin{array}{l}\text { JST, NWT } 400 / 2000+\text { MII } \\
\text { G2-1600 + BVRcIc }\end{array}$ \\
\hline 57917.52285 & 0.0003 & II & $\mathrm{CCD}+\mathrm{R}$ & $74 / 52$ & Lehky M. & $\begin{array}{l}\text { JST, NWT } 400 / 2000+\text { MII } \\
\text { G2-1600 + BVRcIc }\end{array}$ \\
\hline 57917.52318 & 0.0001 & II & $\mathrm{CCD}+\mathrm{V}$ & $70 / 49$ & Lehky M. & $\begin{array}{l}\text { JST, NWT } 400 / 2000+\text { MII } \\
\text { G2-1600 + BVRcIc }\end{array}$ \\
\hline V2635 Oph & & & & & & \\
\hline 57902.47385 & 0.0003 & II & $\mathrm{CCD}+$ Clear & $136 / 40$ & Nosál' P. & $\begin{array}{l}\text { REF } 102 / 500+\text { ATIK 16IC } \\
\text { Mono }\end{array}$ \\
\hline
\end{tabular}

CSS J045746.0+095252 Ori

\begin{tabular}{|c|c|c|c|c|c|}
\hline 58042.58717 & $0.0011 \mathrm{I}$ & CCD + Clear & $128 / 60$ & Mašek M. & $\begin{array}{l}\text { NWT } 150 / 600+\text { CCD MII } \\
\text { G2-1600+ comacorector }\end{array}$ \\
\hline \multicolumn{6}{|l|}{ FF Ori } \\
\hline 58179.32902 & $0.0002 \mathrm{I}$ & $\mathrm{CCD}+$ Clear & $73 / 36$ & Mašek M. & $\begin{array}{l}\text { NWT } 150 / 600+\text { CCD MII } \\
\text { G2-1600 + comacorector }\end{array}$ \\
\hline \multicolumn{6}{|l|}{ V0343 Ori } \\
\hline 57776.34872 & $0.0004 \mathrm{I}$ & $\mathrm{CCD}+\mathrm{V}$ & $159 / 72$ & Šmelcer L. & $\begin{array}{l}\text { Celestron SCT } 355 / 2460 \\
\text { MII G2-1600 }\end{array}$ \\
\hline 57776.34874 & $0.0003 \mathrm{I}$ & $\mathrm{CCD}+\mathrm{R}$ & $143 / 66$ & Šmelcer L. & $\begin{array}{l}\text { Celestron SCT } 355 / 2460+ \\
\text { MII G2-1600 }\end{array}$ \\
\hline
\end{tabular}

V1202 Ori

57776.34176

0.0001 I CCD + Clear $\quad$ 156/92 Mašek M.

NWT 150/600 + CCD MII G2-1600 + comacorector

58042.58255

0.0001 II CCD + Clear 130/58 Mašek M.

NWT 150/600 + CCD MII

G2-1600 + comacorector

V1626 Ori

58175.37087

0.0002 I CCD + Clear 114/58 Mašek M.

NWT $150 / 600+$ CCD MII G2-1600 + comacorector

V1633 Ori

57725.53628

0.0003 II DSLR $\quad 370 / 165$ Walter F.

CZ Mirotar SCT 180/1000+ Canon 350d

V1833 Ori

57405.36501

0.0007 I CCD +Clear 249/207 Jacobsen J.

EQ6, $80 \mathrm{~mm}$ refractor, SXVRH16 


\begin{tabular}{|c|c|c|c|c|c|}
\hline Star & & & & & \\
\hline HJD-2400000 & Error & I/II Method & Points & Observer & Technique \\
\hline 58043.59268 & 0.0013 & I $\quad$ DSLR & $84 / 51$ & $\begin{array}{l}\text { Walter F., Kalá- } \\
\text { šek J. }\end{array}$ & $\begin{array}{l}\text { CZ Mirotar LNS 180/1000 + } \\
\text { Canon 350D (4) }\end{array}$ \\
\hline 58043.59361 & 0.0014 & DSLR & $62 / 38$ & $\begin{array}{l}\text { Walter F., Kalá- } \\
\text { šek J. }\end{array}$ & $\begin{array}{l}\text { CZ Mirotar LNS } 180 / 1000+ \\
\text { Canon 350D (4) }\end{array}$ \\
\hline
\end{tabular}

V1848 Ori

57355.72373

V1853 Ori

57774.41180

V2163 Ori

57749.51234

V2759 Ori

57407.31684

57707.63040

57777.46919

57780.46144

57782.45719

57828.35297

V2762 Ori

57775.29818

V2793 Ori

57725.57700
$0.0001 \quad$ II $\quad \mathrm{CCD}+\mathrm{I}$

0.0006 I CCD + Clear 95/56 Bragagnolo U.

0.0002 I CCD + Clear $276 / 132$ Walter F.

0.0004 I $\quad$ CCD + Clear $121 / 80 \quad$ Jacobsen J.

0.0005 I $\mathrm{CCD}+$ Clear $\quad 123 / 40$ Bragagnolo U.

0.0004 I CCD+Clear 176/102 Urbaník M.

0.0002 I CCD+Clear 221/114 Urbaník M.

0.0002 I $\quad C C D+$ Clear $53 / 27$ Urbaník M.

0.0003 I CCD+Clear 291/169 Urbaník M.

0.0001 I CCD + Clear 85/42 Mašek M.

0.0007 II DSLR

360/213 Walter F.

$1.54 \mathrm{~m}$, Warner + Swasey, NWT, 1540/7480 + Apogee

Sky-Watcher REF 100/900 + QHY9

Kometensucher Zeiss REF 200/1370 + SBIG ST7

EQ6, $80 \mathrm{~mm}$ refractor, $\mathrm{mx} 716$

Sky-Watcher REF 100/900 + QHY9

Sky-Watcher REF 80/520 +

MII G2-8300

Sky-Watcher NWT150/750 +

MII G2-8300

Sky-Watcher NWT 150/750 + MII G2-8300

Sky-Watcher NWT 150/750 + MII G2-8300

NWT 150/600 + CCD MII G2-1600 + comacorector

CZ Mirotar SCT 180/1000 + Canon 350d

ASAS J220925+0808.0 Peg

57981.44393

0.0019 I DSLR

115/52 Walter F.

Tamron SP LNS 62.5/500 +

Canon 350 D 


\begin{tabular}{|c|c|c|c|c|c|}
\hline Star & & & & & \\
\hline HJD-2400000 & Error & I/II Method & Points & Observer & Technique \\
\hline 57981.44079 & 0.0002 & I $\quad$ DSLR & $105 / 45$ & Walter F. & $\begin{array}{l}\text { Tamron SP LNS } 62.5 / 500 \\
\text { Canon } 350 \text { D }\end{array}$ \\
\hline
\end{tabular}

BB Peg

57731.30523

BY Peg

58026.37139

57715.30584

CC Peg

57715.30576
$0.0001 \mathrm{I} \quad \mathrm{CCD}+\mathrm{R}$

215/131 Šmelcer L.

0.0003 I $\quad \mathrm{CCD}+\mathrm{R}$

175/98 Šmelcer L.

0.0003 I CCD + Clear 194/107 Šmelcer L.

0.0002 I CCD+Clear 116/52 Urbaník M.
CSS $222617.1+152225$ Peg

57632.51118

$0.0006 \mathrm{I}$

$\mathrm{CCD}+$ Clear

375/231 Lomoz F.

SNT 254/1016+G2-8300

CSS J215124.3+095921 Peg

57626.43156

$0.0003 \mathrm{I}$

$\mathrm{CCD}+$ Clear

322/100 Lomoz F.

118/47 Nosál' P.

0.0008 I DSLR

57988.44901

CSS J215258.2+141035 Peg

57988.50524

0.0006 I DSLR

147/101 Nosál P.

NWT 150/600 + Canon 20D + MPCC mk.III

CSS J215305.0+143358 Peg

57988.50361

0.0023 I DSLR

147/100 Nosál' P.

NWT 150/600 + Canon 20D + MPCC mk.III

CSS J225026.0 352056 Peg 57655.33811

0.0004 I CCD +Clear 333/83 Lomoz F.

SNT 254/1016+G2-8300

CSS J225239.3+345309 Peg 57655.37298 0.0009 I CCD + Clear 496/152 Lomoz F.

134/99 Nosál P.

Celestron SCT 280/1765 + MII G2-4000

Celestron SCT 280/1765 + MII G2-4000

STN 254/1016+G2-8300

NWT 150/600 + Canon 20D + MPCC mk.III

CSS J225846.2+262348 Peg

57715.36454

0.0032 I DSLR 
\begin{tabular}{l} 
Star \\
HJD-2400000 \\
\hline 57715.27451 \\
CzeV444 Peg
\end{tabular}

57715.33832

57715.34130

EU Peg

57715.27639

58025.35483

HS $2231+2441$ Peg 57980.35413

57980.40984

57980.46536

KW Peg

57981.51455

57981.51477

NSVS $11706081 \mathrm{Peg}$ 57988.43705

NSVS 11707553 Peg 57626.39760

NSVS 11725339 Peg 57981.53095

NSVS 8970952 Peg 57715.35269

$\begin{array}{lllll}0.0003 & \text { I } & \text { DSLR } & 137 / 37 & \text { Nosál’ P. } \\ 0.0001 & \text { I } & \text { CCD }+R & 117 / 74 & \text { Hanžl D. }\end{array}$

0.0011 II CCD + Clear 119/89 Šmelcer L.

0.0011 II $\quad \mathrm{CCD}+\mathrm{R} \quad 79 / 65 \quad$ Šmelcer L.

\begin{tabular}{lllll} 
Error & I/II & Method & Points & Observer \\
\hline 0.0012 & I & DSLR & $137 / 36$ & Nosál' P.
\end{tabular}

Technique

NWT 150/600 + Canon 20D + MPCC mk.III

Celestron SCT 280/1765 + MII G2-4000

Celestron SCT 280/1765 + MII G2-4000

NWT 150/600 + Canon 20D + MPCC mk.III

DATEL, NWT 200/800 + MII G2 8300

Sky-Watcher NWT 254/1200 + MII G2-402

0.0003 II $\mathrm{CCD}+\mathrm{R} \quad 228 / 94 \quad$ Šmelcer L.

Sky-Watcher NWT 254/1200 + MII G2-402

$0.0001 \mathrm{I} \quad \mathrm{CCD}+\mathrm{R} \quad 228 / 151$ Šmelcer L.

Sky-Watcher NWT 254/1200

+ MII G2-402

Sky-Watcher NWT 254/1200 + MII G2-402

Sky-Watcher NWT 254/1200 + MII G2-402

0.0017 I DSLR 149/55 Nosál’ P.

NWT $150 / 600+$ Canon 20D + MPCC mk.III

0.0003 I CCD + Clear 250/59 Lomoz F.

SNT 254/1016+G2-8300

0.0018 I DSLR $\quad 120 / 102$ Walter F.

Tamron SP LNS 62.5/500 + Canon 350 D

NWT $150 / 600+$ Canon 20D + MPCC mk.III 
Star

HJD-2400000

Error I/II Method

Points Observer

Technique

NSVS 9010274 Peg

57755.29098

0.0004 I CCD + Clear $\quad 171 / 89 \quad$ Urbaník M.

Sky-Watcher REF 80/520 + MII G2-8300

UX Peg

57728.27448

0.0002 I $\quad$ CCD + Clear 212/121 Bragagnolo U.

Sky-Watcher REF 100/900 + QHY9

V0357 Peg

58021.61738

V0404 Peg

57981.36227

V0436 Peg

57988.45425

V0449 Peg

57715.31424

57715.31591

V0462 Peg

57988.40902

V0463 Peg

58019.36993

V0473 Peg

57989.39648

V0477 Peg

57997.31926

57997.47861
0.007 I DSLR

151/11 Školník V.

0.0001 I Photometer + V60/32 Persha G.

Meade RCT 250/2500 + Optec SSP-5a

Tamron LNS 44/300 + Canon $600 \mathrm{D}$

REF $102 / 500+$ ATIK 16 IC mono

Celestron SCT 280/1765 + MII G2-4000

Celestron SCT 280/1765 + MII G2-4000

NWT $150 / 600+$ Canon 20D + MPCC mk.III

REF $102 / 500+$ ATIK 16 IC mono

NWT $150 / 600+$ Canon 20D + MPCC mk.III

REF $102 / 500+$ ATIK 16 IC mono

NWT 150/600 + Canon 20D + MPCC mk.III 


\begin{tabular}{|c|c|c|c|c|c|}
\hline Star & & & & & \\
\hline HJD-2400000 & Error & I/II Method & Points & Observer & Technique \\
\hline 57755.32214 & 0.0002 & I $\quad$ CCD + Clear & $171 / 126$ & Urbaník M. & $\begin{array}{l}\text { Sky-Watcher REF } 80 / 520+ \\
\text { MII G2-8300 }\end{array}$ \\
\hline
\end{tabular}

V0628 Peg

57715.21293

57715.21535

V0669 Peg 57755.32008

BP Per 57720.32444

CzeV1280 Per 58072.28721

CzeV686 Per 58072.34142

58084.32420

DK Per

57966.53012

57966.53021

57966.53028

IK Per

57645.53165

IQ Per

58106.39045

IU Per

57644.50843

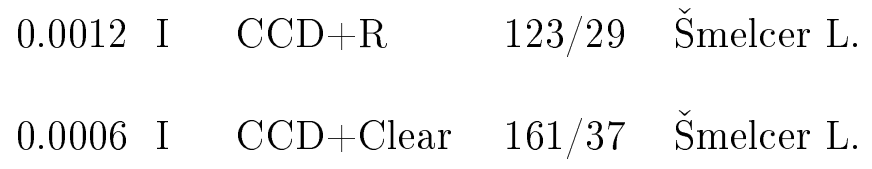

0.0006 I CCD+Clear 162/115 Urbaník M.
Celestron SCT 280/1765 + MII G2-4000

Celestron SCT 280/1765 + MII G2-4000

Sky-Watcher REF 80/520 + MII G2-8300

HK25, NWT 250/1000 SBIG ST-7 + Rc

NWT 250/1000 + CCD MII G2-8300
0.0003 I CCD+Clear 137/101 Mašek M., Tylšar M.

$0.001 \quad \mathrm{I} \quad \mathrm{CCD}+$ Clear $\quad 45 / 17$
Mašek M., Tylšar M.

NWT 250/1000 + CCD MII G2-8300

NWT 250/1000 + CCD MII G2-8300

JST, NWT 400/2000 + MII G2-1600 + BVRcIc JST, NWT 400/2000 + MII G2-1600 + BVRcIc JST, NWT 400/2000 + MII G2-1600 + BVRcIc

0.0001 I $\quad \mathrm{CCD}+\mathrm{V} \quad 32 / 16 \quad$ Lehky M.

0.0001 I $\quad \mathrm{CCD}+\mathrm{I}$

$0.0001 \mathrm{I} \quad \mathrm{CCD}+\mathrm{R}$
33/17 Lehky M.

35/17 Lehky M.

\section{KN Per}




\begin{tabular}{|c|c|c|c|c|c|}
\hline Star & & & & & \\
\hline HJD-2400000 & Error & I/II Method & Points & Observer & Technique \\
\hline 57755.41814 & 0.0009 & I $\quad$ CCD + Clear & $168 / 102$ & Bragagnolo U. & $\begin{array}{l}\text { Sky-Watcher REF } 100 / 900+ \\
\text { QHY9 }\end{array}$ \\
\hline
\end{tabular}

KR Per

57706.40958

58112.31528

KW Per

57782.22577

NP Per

58026.61466

58026.61507

NSVS 4147261 Per 57750.40671

NSVS 4220985 Per 57645.62812

NSVS 4307145 Per 57799.36275

NZ Per

57755.26998

QW Per

58045.38815

RT Per

57726.34832

58111.55398

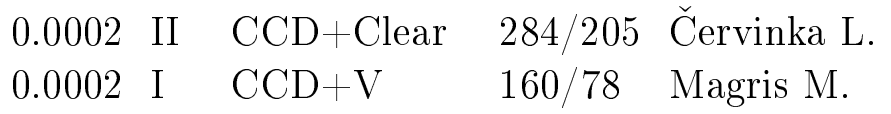

$0.0002 \mathrm{I} \quad \mathrm{CCD}+\mathrm{R} \quad 219 / 10 \quad$ Vrašták M

0.0004 I DSLR

0.0004 I DSLR

$70 / 25$

Walter F., Kalášek J.

$83 / 31$

Walter F., Kalášek J.

CZ Mirotar LNS 180/1000+ Canon 350D (4)

CZ Mirotar LNS 180/1000 + Canon 350D (4)

NWT 280/1500+MII G2-1600, $80 / 400+$ G1-0300

0.0004 I $\quad$ CCD + Clear $\quad 139 / 60 \quad$ Bragagnolo U.

Sky-Watcher REF 100/900 + QHY9

0.0008 I $\quad$ CCD + Clear 344/306 Červinka L.

R102/500 + Atik 314L +

0.0018 I DSLR

128/90 Sergey I.

Lens Jupiter-21 + Canon 400D

$0.0004 \mathrm{I} \quad \mathrm{CCD}+$ Clear $91 / 33 \quad$ Magris $\mathrm{M}$.

Achromatic refractor 120/600, CCD Orion Starshoo

NWT 355/1600+MII G2-1600, $80 / 400+$ G1-0301

$0.0001 \mathrm{I} \quad \mathrm{CCD}+\mathrm{R} \quad 72 / 35 \quad$ Vrašták M.

NWT 200/1000 + Atik 314L+ Achromatic refractor 120/600,

RY Per

57730.26597
0.0003 II CCD + Clear $225 / 179$ Červinka L.

$0.0003 \mathrm{I} \quad \mathrm{CCD}+\mathrm{V} \quad 261 / 206$ Magris $\mathrm{M}$.
CCD Orion Starshoo

Sky-Watcher REF 80/520 + MII G2-8300 


\begin{abstract}
Star
HJD-2400000

57994.36114

SERIV 103 Per
\end{abstract}

57994.40601

SERIV 105 Per

57997.38057

SERIV 106 Per

57997.49702

SERIV 107 Per

58019.44377

V0432 Per

57750.40468

V0723 Per

57799.35804

V0737 Per

57722.34726

V0740 Per

57780.37385

57780.37393

V0871 Per

57714.42064

V0873 Per

57995.57289

57995.57290
0.0023 I DSLR

0.0007 I DSLR

98/47 Sergey I.

0.0021 I DSLR

106/85 Sergey I.

0.0005 I DSLR $\quad 126 / 85$ Sergey I.

0.0002 II CCD + Clear $141 / 58$ Bragagnolo U.

0.0003 I CCD + Clear 234/145 Bragagnolo U.

0.0002 I CCD + Clear 318/150 Lehky M.

0.0002 I DSLR $\quad 256 / 87$ Walter F.

0.0001 I CCD + Clear 289/122 Walter F.

$0.0002 \mathrm{I} \quad \mathrm{CCD}+\mathrm{R} \quad 640 / 105$ Šmelcer L.
Sky-Watcher REF 100/900 QHY9

Sky-Watcher REF 100/900 + QHY9

Technique

Newton $200 \mathrm{~mm}(1: 5)+$ Canon $400 \mathrm{D}$

Newton 200 mm (1:5) + Canon 400D

Newton 200 mm (1:5) + Canon 400D

Newton 200 mm (1:5) + Canon 400D

Newton 200 mm (1:5) + Canon 400D

HK25, NWT 250/1000

SBIG ST-7 + Rc

CZ Mirotar SCT 180/1000, Canon 350d

Kometensucher Zeiss REF 200/1370 + SBIG ST7

Celestron SCT 280/1765 + MII G2-4000

JST, NWT 400/2000 + MII G2-1600 + BVRcIc

JST, NWT 400/2000 + MII

G2-1600 + BVRcIc 


\begin{tabular}{|c|c|c|c|c|c|}
\hline \multicolumn{6}{|l|}{ Star } \\
\hline HJD-2400000 & Error & I/II Method & Points & Observer & Technique \\
\hline 57995.57306 & 0.0001 & I $\quad \mathrm{CCD}+\mathrm{V}$ & $39 / 24$ & Lehky M. & $\begin{array}{l}\text { JST, NWT 400/2000 + MII } \\
\text { G2-1600 + BVRcIc }\end{array}$ \\
\hline 57995.57321 & 0.0001 & $\mathrm{CCD}+\mathrm{B}$ & $42 / 26$ & Lehky M. & $\begin{array}{l}\text { JST, NWT } 400 / 2000+\text { MII } \\
\text { G2- } 1600+\text { BVRcIc }\end{array}$ \\
\hline
\end{tabular}

V0887 Per

57690.64377

58093.44313
0.0004 I CCD + Clear $167 / 63 \quad$ Bragagnolo U.

0.0004 II CCD +Clear 221/124 Bragagnolo U.
Sky-Watcher REF 100/900 QHY9

Sky-Watcher REF 100/900 + QHY9

JST, NWT 400/2000 + MII G2-1600 + BVRcIc

JST, NWT 400/2000 + MII G2-1600 + BVRcIc

JST, NWT 400/2000 + MII G2-1600 + BVRcIc

JST, NWT 400/2000 + MII G2-1600 + BVRcIc

JST, NWT 400/2000 + MII G2-1600 + BVRcIc

JST, NWT 400/2000 + MII G2-1600 + BVRcIc

JST, NWT 400/2000 + MII G2-1600 + BVRcIc

JST, NWT 400/2000 + MII G2-1600 + BVRcIc

JST, NWT 400/2000 + MII G2-1600 + BVRcIc

JST, NWT 400/2000 + MII

G2-1600 + BVRcIc

JST, NWT 400/2000 + MII G2-1600 + BVRcIc

JST, NWT 400/2000 + MII G2-1600 + BVRcIc

JST, NWT 400/2000 + MII G2-1600 + BVRcIc

JST, NWT 400/2000 + MII G2-1600 + BVRcIc

JST, NWT 400/2000 + MII G2-1600 + BVRcIc

JST, NWT 400/2000 + MII G2-1600 + BVRcIc

JST, NWT 400/2000 + MII G2-1600 + BVRcIc

JST, NWT 400/2000 + MII G2-1600 + BVRcIc 


\begin{tabular}{|c|c|c|c|c|c|}
\hline Star & & & & & \\
\hline HJD-2400000 & Error & $\mathrm{I} / \mathrm{I}$ & Method & Points & Observer \\
\hline 56167.36002 & 0.0024 & $\mathrm{I}$ & $\mathrm{CCD}+\mathrm{I}$ & $72 / 13$ & Lehky M. \\
\hline 56167.36151 & 0.0014 & $\mathrm{I}$ & $\mathrm{CCD}+\mathrm{I}$ & $74 / 15$ & Lehky M. \\
\hline 56167.36384 & 0.0011 & $\mathrm{I}$ & $\mathrm{CCD}+\mathrm{R}$ & $71 / 15$ & Lehky M. \\
\hline 56167.36489 & 0.0009 & $\mathrm{I}$ & $\mathrm{CCD}+\mathrm{V}$ & $68 / 12$ & Lehky M. \\
\hline 56167.60216 & 0.0022 & II & $\mathrm{CCD}+\mathrm{B}$ & $70 / 63$ & Lehky M. \\
\hline 56167.60920 & 0.0025 & II & $\mathrm{CCD}+\mathrm{R}$ & $79 / 74$ & Lehky M. \\
\hline 56167.61092 & 0.0016 & II & $\mathrm{CCD}+\mathrm{I}$ & $74 / 68$ & Lehky M. \\
\hline 56167.61183 & 0.001 & II & $\mathrm{CCD}+\mathrm{V}$ & $68 / 64$ & Lehky M. \\
\hline 56180.35858 & 0.0017 & II & $\mathrm{CCD}+\mathrm{B}$ & $66 / 15$ & Lehky M. \\
\hline 56180.36698 & 0.0015 & II & $\mathrm{CCD}+\mathrm{R}$ & $82 / 21$ & Lehky M. \\
\hline 56180.36803 & 0.001 & II & $\mathrm{CCD}+\mathrm{V}$ & $82 / 19$ & Lehky M. \\
\hline 56180.36854 & 0.0019 & II & $\mathrm{CCD}+\mathrm{I}$ & $82 / 19$ & Lehky M. \\
\hline 56180.37069 & 0.0015 & II & $\mathrm{CCD}+\mathrm{R}$ & $79 / 20$ & Lehky M. \\
\hline 56180.37253 & 0.0016 & II & $\mathrm{CCD}+\mathrm{I}$ & $76 / 20$ & Lehky M. \\
\hline 56180.60213 & 0.001 & I & $\mathrm{CCD}+\mathrm{V}$ & $82 / 74$ & Lehky M. \\
\hline 56180.61017 & 0.0019 & I & $\mathrm{CCD}+\mathrm{I}$ & $82 / 76$ & Lehky M. \\
\hline
\end{tabular}

Technique

JST, NWT $400 / 2000+$ MII G2-1600 + BVRcIc

JST, NWT 400/2000 + MII G2-1600 + BVRcIc

JST, NWT 400/2000 + MII G2-1600 + BVRcIc

JST, NWT 400/2000 + MII G2-1600 + BVRcIc

JST, NWT 400/2000 + MII G2-1600 + BVRcIc

JST, NWT $400 / 2000+$ MII

G2-1600 + BVRcIc

JST, NWT 400/2000 + MII G2-1600 + BVRcIc

JST, NWT 400/2000 + MII

G2-1600 + BVRcIc

JST, NWT 400/2000 + MII G2-1600 + BVRcIc

JST, NWT 400/2000 + MII G2-1600 + BVRcIc

JST, NWT 400/2000 + MII G2-1600 + BVRcIc

JST, NWT $400 / 2000+$ MII G2-1600 + BVRcIc

JST, NWT 400/2000 + MII G2-1600 + BVRcIc

JST, NWT $400 / 2000+$ MII G2-1600 + BVRcIc

JST, NWT 400/2000 + MII G2-1600 + BVRcIc

JST, NWT 400/2000 + MII G2-1600 + BVRcIc

VSX J013555.9+541142 Per

$\begin{array}{llllll}55834.52746 & 0.0013 & \text { I } & \text { CCD }+ \text { B } & 106 / 92 & \text { Lehky M. } \\ 55834.52993 & 0.0007 & \text { I } & \text { CCD }+ \text { R } & 107 / 96 & \text { Lehky M. } \\ 55834.53117 & 0.0012 & \text { I } & \text { CCD }+\mathrm{V} & 121 / 105 & \text { Lehky M. } \\ 56155.45884 & 0.0029 & \text { I } & \text { CCD }+ \text { B } & 39 / 13 & \text { Lehky M. } \\ 56155.45924 & 0.0014 & \text { I } & \text { CCD }+ \text { R } & 62 / 29 & \text { Lehky M. } \\ 56155.45968 & 0.0014 & \text { I } & \text { CCD }+\mathrm{I} & 67 / 31 & \text { Lehky M. } \\ 56155.46121 & 0.0012 & \text { I } & \text { CCD }+ \text { V } & 70 / 34 & \text { Lehky M. }\end{array}$

JST, NWT 400/2000 + MII G2-1600 + BVRcIc

JST, NWT 400/2000 + MII G2-1600 + BVRcIc

JST, NWT 400/2000 + MII G2-1600 + BVRcIc

JST, NWT 400/2000 + MII

G2-1600 + BVRcIc

JST, NWT 400/2000 + MII G2-1600 + BVRcIc

JST, NWT 400/2000 + MII G2-1600 + BVRcIc

JST, NWT 400/2000 + MII G2-1600 + BVRcIc 


\begin{tabular}{|c|c|c|c|c|c|}
\hline \multicolumn{6}{|l|}{ Star } \\
\hline HJD-2400000 & Error & $\mathrm{I} / \mathrm{II}$ & Method & Points & Observer \\
\hline 56155.46311 & 0.0007 & I & $\mathrm{CCD}+\mathrm{R}$ & $69 / 35$ & Lehky M. \\
\hline 56159.54946 & 0.0022 & II & $\mathrm{CCD}+\mathrm{V}$ & $61 / 47$ & Lehky M. \\
\hline 56159.55167 & 0.0029 & II & $\mathrm{CCD}+\mathrm{B}$ & $46 / 37$ & Lehky M. \\
\hline 56159.55360 & 0.0018 & II & $\mathrm{CCD}+\mathrm{R}$ & $68 / 55$ & Lehky M. \\
\hline 56159.55714 & 0.0027 & II & $\mathrm{CCD}+\mathrm{I}$ & $66 / 53$ & Lehky M. \\
\hline 56167.45198 & 0.0012 & $\mathrm{I}$ & $\mathrm{CCD}+\mathrm{V}$ & $75 / 36$ & Lehky M. \\
\hline 56167.45291 & 0.0011 & $\mathrm{I}$ & $\mathrm{CCD}+\mathrm{I}$ & $69 / 30$ & Lehky M. \\
\hline 56167.45368 & 0.001 & $\mathrm{I}$ & $\mathrm{CCD}+\mathrm{R}$ & $77 / 38$ & Lehky M. \\
\hline 56167.46294 & 0.0018 & $\mathrm{I}$ & $\mathrm{CCD}+\mathrm{B}$ & $58 / 28$ & Lehky M. \\
\hline 56180.52600 & 0.0021 & I & $\mathrm{CCD}+\mathrm{B}$ & $44 / 31$ & Lehky M. \\
\hline 56180.52714 & 0.0011 & $\mathrm{I}$ & $\mathrm{CCD}+\mathrm{V}$ & $80 / 54$ & Lehky M. \\
\hline 56180.52728 & 0.0011 & $\mathrm{I}$ & $\mathrm{CCD}+\mathrm{I}$ & $84 / 57$ & Lehky M. \\
\hline 56180.52833 & 0.0008 & $\mathrm{I}$ & $\mathrm{CCD}+\mathrm{R}$ & $86 / 61$ & Lehky M. \\
\hline
\end{tabular}

XZ Per

57763.48215

57763.48233

$0.0001 \mathrm{I} \quad \mathrm{CCD}+\mathrm{R} \quad 85 / 28 \quad$ Šmelcer L.

Celestron SCT 280/1765 + MII G2-4000

0.0001 I $\quad$ CCD + Clear $\quad 92 / 28 \quad$ Šmelcer L.

Celestron SCT 280/1765 + MII G2-4000

YZ Phe

57696.54639

0.0001 II $\quad \mathrm{CCD}+\mathrm{V} \quad 76 / 53 \quad$ Tornatore $\mathrm{M}$.

Celestron SCT 355,6/1816 + Sbig ST7 + Reductor f

RZ PsA

57721.59217

$0.0001 \mathrm{I} \quad \mathrm{CCD}+\mathrm{R} \quad 88 / 39 \quad$ Mašek M.

FRAM, Nikkor LNS 106/300 + G4-16000 


\begin{tabular}{|c|c|c|c|c|c|}
\hline Star & & & & & \\
\hline HJD-2400000 & Error & I/II Method & Points & Observer & Technique \\
\hline 57615.64563 & 0.0002 & $\mathrm{I} \quad \mathrm{CCD}+\mathrm{R}$ & $182 / 71$ & Quinones C. & $\begin{array}{l}\text { 1.54m, Warner }+ \text { Swasey, } \\
\text { NWT, } 1540 / 7480+\text { Apogee }\end{array}$ \\
\hline
\end{tabular}

2MASS J23334239-012939.6 Psc

\begin{tabular}{|c|c|c|c|c|c|c|}
\hline 57642.55232 & 0.0003 & $\mathrm{I}$ & CCD + Clear & $571 / 437$ & Lomoz F. & SNT $254 / 1016+$ G2-8300 \\
\hline \multicolumn{7}{|c|}{ ASAS J005428+1150.7 Psc } \\
\hline 57728.24881 & 0.0003 & $\mathrm{I}$ & DSLR & $110 / 55$ & Nosál' P. & $\begin{array}{l}\text { NWT } 150 / 600+\text { Canon } 20 \mathrm{D}+ \\
\text { MPCC mk.III }\end{array}$ \\
\hline \multicolumn{7}{|l|}{ CP Psc } \\
\hline 57996.55896 & 0.0003 & I & $\mathrm{CCD}+\mathrm{I}$ & $48 / 26$ & Lehky M. & $\begin{array}{l}\text { JST, NWT } 400 / 2000+\text { MII } \\
\text { G2-1600 + BVRcIc }\end{array}$ \\
\hline 57996.55918 & 0.0003 & $\mathrm{I}$ & $\mathrm{CCD}+\mathrm{B}$ & $46 / 26$ & Lehky M. & $\begin{array}{l}\text { JST, NWT } 400 / 2000+\text { MII } \\
\text { G2-1600 + BVRcIc }\end{array}$ \\
\hline 57996.55926 & 0.0003 & $\mathrm{I}$ & $\mathrm{CCD}+\mathrm{R}$ & $46 / 26$ & Lehky M. & $\begin{array}{l}\text { JST, NWT } 400 / 2000+\text { MII } \\
\text { G2-1600 + BVRcIc }\end{array}$ \\
\hline 57996.55960 & 0.0002 & $\mathrm{I}$ & $\mathrm{CCD}+\mathrm{V}$ & $38 / 23$ & Lehky M. & $\begin{array}{l}\text { JST, NWT } 400 / 2000+\text { MII } \\
\text { G2-1600 + BVRcIc }\end{array}$ \\
\hline 58085.48041 & 0.0001 & $\mathrm{I}$ & $\mathrm{CCD}+$ Clear & $254 / 141$ & Bragagnolo U. & $\begin{array}{l}\text { Sky-Watcher REF 100/900 + } \\
\text { QHY9 }\end{array}$ \\
\hline
\end{tabular}

CzeV526 Psc

57646.55446

0.0006 I CCD + Clear $87 / 50 \quad$ Mašek M.

NWT $150 / 600+$ CCD MII

58044.34799

0.0009 II CCD + Clear 157/52 Mašek M.

G2-1600 + comacorector

NWT $150 / 600+$ CCD MII

G2-1600 + comacorector

EE Psc

58035.52961 0.0007 I $\quad$ CCD + R $114 / 36$ Hanžl D.

DATEL, NWT 200/800 + MII G2 8300

ET Psc

57728.29933

0.0006 I DSLR

115/91 Nosál' P.

NWT $150 / 600+$ Canon 20D + MPCC mk.III

58041.51385

0.0012 I CCD + Clear 211/23 Hladík B.

LNS $38 / 135+$ ATIK 320E + MuniWin

EW Psc

57968.56426

0.0008 II CCD + Clear $111 / 74 \quad$ Nosál P.

58041.52205

$0.0004 \mathrm{I} \quad \mathrm{CCD}+\mathrm{V} \quad 92 / 70$

Gudmundsson

S.

0.0006 II $\mathrm{CCD}+\mathrm{V} \quad 73 / 22 \quad$ Gudmundsson

REF $102 / 500+$ ATIK 16 IC mono

$400 \mathrm{~mm} \quad \mathrm{LX} 200 \mathrm{SCT} / \mathrm{SBIG}$ STL11k CCD

$400 \mathrm{~mm}$ LX200 SCT/SBIG STL11k CCD 


\begin{tabular}{|c|c|c|c|c|c|}
\hline Star & & & & & \\
\hline HJD-2400000 & Error & I/II Method & Points & Observer & Technique \\
\hline 58042.48873 & 0.0005 & I $\quad \mathrm{CCD}+\mathrm{V}$ & $73 / 58$ & $\begin{array}{l}\text { Gudmundsson } \\
\text { S. }\end{array}$ & $\begin{array}{l}300 \mathrm{~mm} \text { LX200 SCT/SBIG } \\
\text { STL11k CCD }\end{array}$ \\
\hline 58043.45180 & 0.0005 & $\mathrm{CCD}+\mathrm{V}$ & $62 / 34$ & $\begin{array}{l}\text { Gudmundsson } \\
\text { S. }\end{array}$ & $\begin{array}{l}400 \mathrm{~mm} \text { LX200 SCT/SBIG } \\
\text { STL11k CCD }\end{array}$ \\
\hline
\end{tabular}

FU Psc

$58041.41447 \quad 0.0002$ I $\quad$ CCD + Clear $\quad 317 / 135$ Nosál P.

REF $102 / 500+$ ATIK 16IC mono

GSC 05251-00240 Psc

57642.42637

57642.58776

57642.58790

57666.43877

GT Psc

57726.25327

0.0013 I DSLR

0.0004 II $\mathrm{CCD}+$ Clear

0.0003 I $\mathrm{CCD}+$ Clear

0.0003 I $\mathrm{CCD}+$ Clear

0.0003 II $\mathrm{CCD}+$ Clear

$590 / 225$ Lomoz F.

590/516 Lomoz F.

590/517 Lomoz F.

411/338 Lomoz F.

95/37 Nosál P.

NWT 150/600 + Canon 20D +

MPCC mk.III

HL Psc

57659.58587

58044.26994

0.001 I DSLR

0.0004 II $\mathrm{CCD}+$ Clear

199/174 Nosál P.

243/42 Ehrenberger R.

HN Psc

57729.25784

0.0007 II DSLR

108/34 Nosál P.

0.0003 II $\quad \mathrm{CCD}+\mathrm{V} \quad 88 / 53 \quad$ Šuchaň J.

57994.52016

58097.32904

0.0002 I $\quad$ CCD + Clear $\quad 97 / 29$

0.0004 I $\quad$ CCD + Clear $\quad 97 / 81$

Gudmundsson

S.

Gudmundsson

S.

2MASS J23334239-0129396 Psc

57642.55233

0.0003

EW Psc

58041.40721

0.0007 II $\quad \mathrm{CCD}+\mathrm{V}$

$92 / 29$

Gudmundsson S.
STN 254/1016+G2-8300

SNT 254/1016+G2-8300

SNT 254/1016+G2-8300

STN 254/1016+G2-8300
NWT 150/600 + Canon 20D + MPCC mk.III

NWT 150/600+MII G2-8300

NWT 150/600 + Canon 20D + MPCC mk.III

Sky-Watcher NWT 250/1200 + Algol 0402

$400 \mathrm{~mm}$ LX200 SCT/SBIG STL11k CCD

$400 \mathrm{~mm}$ LX200 SCT/SBIG STL11k CCD

SNT 254/1016+G2-8300

$400 \mathrm{~mm}$ LX200 SCT/SBIG STL11k CCD 
Star

HJD-2400000

Error I/II Method

Points Observer

Technique

ASAS J072040-4509.4 Pup

57770.81282

$0.0012 \mathrm{I} \quad \mathrm{CCD}+\mathrm{R} \quad 51 / 43 \quad$ Mašek M.

FRAM, Nikkor LNS 106/300

+ G4-16000

ASAS J081347-4034.2 Pup

58085.68843

$0.0015 \mathrm{I} \quad \mathrm{CCD}+\mathrm{R} \quad 87 / 34 \quad$ Mašek M.

FRAM, Nikkor LNS 106/300

+ G4-16000

ASAS J082010-4237.4 Pup
58085.70145
0.0013
$\mathrm{CCD}+\mathrm{R}$
87/42 Mašek M.
FRAM, Nikkor LNS 106/300
+ G4-16000

EN Pup

58084.70821

SW Pup

57748.67785

V0654 Pup

57771.79510

BE Scl

58083.62626

0.0001 I $\quad$ CCD + Clear $\quad 209 / 91$

Colazo C., Me- Telescopio STC $16+$ CCD lia R., Starck M. QHY-174

AO Ser

57981.35618

57981.35619

$0.0001 \mathrm{I} \quad \mathrm{CCD}+\mathrm{V} \quad 86 / 37 \quad$ Śmelcer L.

$0.0001 \mathrm{I} \quad \mathrm{CCD}+\mathrm{R} \quad 88 / 37 \quad \check{\text { Śmelcer } \mathrm{L} .}$

AQ Ser

58179.63077

0.0002 I CCD + Clear 76/47 Mašek M.
FRAM, Nikkor LNS 106/300

+ G4-16000

FRAM, Nikkor LNS 106/300

+ G4-16000
Sky-Watcher NWT 254/1200 + MII G2-402

Sky-Watcher NWT 254/1200

+ MII G2-402

NWT 150/600 + CCD MII G2-1600 + comacorector

\section{AS Ser}




\begin{tabular}{|c|c|c|c|c|c|}
\hline Star & & & & & \\
\hline HJD-2400000 & Error & I/II Method & Points & Observer & Technique \\
\hline 58178.62921 & 0.0004 & II $\quad \mathrm{CCD}+$ Clear & $69 / 35$ & Mašek M. & $\begin{array}{l}\text { NWT } 150 / 600+\text { CCD MII } \\
\text { G2-1600 + comacorector }\end{array}$ \\
\hline
\end{tabular}

ASAS J154033+0218.0 Ser

$\begin{array}{lllllll}57845.62088 & 0.0005 & \text { I } & \text { CCD }+ \text { Clear } & 54 / 37 & \text { Mašek M. } & \begin{array}{l}\text { NWT } 150 / 600+\text { CCD MII } \\ \text { G2-1600+ comacorector }\end{array} \\ 58178.67576 & 0.0006 & \text { I } & \text { CCD }+ \text { Clear } & 69 / 56 & \text { Mašek M. } & \begin{array}{l}\text { NWT 150/600 + CCD MII } \\ \text { G2-1600+ comacorector }\end{array}\end{array}$

CSS J153817.8+015243 Ser

$57841.52508 \quad 0.0012$ I $\quad$ CCD + Clear $\quad 80 / 19 \quad$ Mašek M.

NWT $150 / 600+$ CCD MII G2-1600 + comacorector

LINEAR 14832957

Ser

57841.52489

0.0009 I CCD +Clear 79/19 Mašek M.

NWT 150/600 + CCD MII G2-1600 + comacorector

NSVS 10653195 Ser

$\begin{array}{llllll}57884.42347 & 0.0001 & \text { I } & \text { CCD }+\mathrm{R} & 138 / 60 & \text { Śmelcer L. } \\ 57884.42349 & 0.0001 & \text { I } & \text { CCD }+ \text { V } & 135 / 58 & \text { Śmelcer L. } \\ 57891.43229 & 0.0001 & \text { II } & \text { CCD }+\mathrm{V} & 75 / 25 & \text { Šmelcer L. } \\ 57891.43235 & 0.0001 & \text { II } & \text { CCD }+ \text { R } & 76 / 27 & \text { Šmelcer L. }\end{array}$

Sky-Watcher NWT 254/1200 + MII G2-402

Sky-Watcher NWT 254/1200 + MII G2-402

Sky-Watcher NWT 254/1200 + MII G2-402

Sky-Watcher NWT 254/1200 + MII G2-402

OU Ser

57891.45195

0.0008 I $\quad$ CDD + Clear $\quad 132 / 64$ Hladík B.

57891.45350

0.0001 I $\quad C C D+R \quad$ 248/129 Mašek M.

57895.45882

0.0002 II $\quad \mathrm{CCD}+\mathrm{R} \quad 84 / 54 \quad$ Mašek M.

58180.64874

0.0002 II $\quad \mathrm{CCD}+\mathrm{R} \quad$ 203/135 Mašek M.

LNS 38/135 + ATIK 320E + MuniWin

NWT 150/600 + CCD MII G2-1600 + comacorector

NWT 150/600 + CCD MII $\mathrm{G} 2-1600+$ comacorector NWT $150 / 600+$ CCD MII G2-1600 + comacorector

TYC 5112-252-1 Ser 57935.51523

0.0004 II CCD+Clear 463/408 Červinka L.

57938.44899

0.0003 I $\quad$ CCD +B $\quad 137 / 95 \quad$ Šmelcer L.

57941.37896

0.0004 I $\quad C C D+B \quad 94 / 16 \quad$ Śmelcer L.

57966.77958

0.0006 I $\quad \mathrm{CCD}+\mathrm{V} \quad$ 231/198 Mašek M.

Tamron 55-200@135mm, Atik $320 \mathrm{E}$ mono

Celestron SCT 280/1765 + MII G2-4000

Celestron SCT 280/1765 + MII G2-4000

FRAM, Meade SCT 300/3000 + MII G2-1600 


\begin{tabular}{|c|c|c|c|c|c|}
\hline Star & & & & & \\
\hline HJD-2400000 & Error & I/II Method & Points & Observer & Technique \\
\hline 57966.78003 & 0.0003 & I $\quad \mathrm{CCD}+\mathrm{R}$ & $187 / 149$ & Mašek M. & $\begin{array}{l}\text { FRAM, Meade SCT 300/3000 } \\
+ \text { MII G2-1600 }\end{array}$ \\
\hline 57980.45666 & 0.0008 & $\mathrm{CCD}+\mathrm{B}$ & $174 / 146$ & Šmelcer L. & $\begin{array}{l}\text { Celestron SCT } 280 / 1765+ \\
\text { MII G2-4000 }\end{array}$ \\
\hline 57981.43553 & 0.001 & $\mathrm{CCD}+\mathrm{B}$ & 199/136 & Šmelcer L. & $\begin{array}{l}\text { Celestron SCT 280/1765 + } \\
\text { MII G2-4000 }\end{array}$ \\
\hline
\end{tabular}

V0385 Ser

57839.63088

0.0003 I $\quad C C D+$ Clear 63/51 Mašek M.

NWT $150 / 600+$ CCD MII

G2-1600 + comacorector

V0413 Ser

57926.49267

0.0011 I CCD + Clear 159/101 Červinka L.

Tamron 55-200@135mm, Atik $320 \mathrm{E}$ mono

V0554 Ser

57935.51266

0.003 II CCD + Clear 250/218 Červinka L.

Tamron 55-200@135mm, Atik $320 \mathrm{E}$ mono

CSS J102008.0-095827 Sex

\begin{tabular}{|c|c|c|c|c|}
\hline 57798.52029 & 0.001 & CCD +Clear & 402/244 Lomoz F. & SNT 254/1016+G2-8300 \\
\hline 58173.50422 & $0.0006 \mathrm{I}$ & CCD+Clear & 328/169 Lomoz F. & Newton $300 / 1200+$ ST2000XM \\
\hline 58173.50511 & $0.0004 \mathrm{I}$ & CCD + Clear & 479/282 Lomoz F. & SNT254/1016+G2-8300 \\
\hline
\end{tabular}

CSS J102120.2-094738 Sex

$\begin{array}{lllllll}57798.51068 & 0.001 & \text { I } & \text { CCD +Clear } & 299 / 170 & \text { Lomoz F. } & \text { SNT 254/1016+G2-8300 } \\ 58173.42443 & 0.0011 & \text { I } & \text { CCD +Clear } & 329 / 73 & \text { Lomoz F. } & \text { SNT254/1016+G2-8300 }\end{array}$

ASAS J191751+1822.7 Sge

$57923.36635 \quad 0.0002$ I $\quad$ CCD + Clear $\quad 261 / 39$ Urbaník M.

Sky-Watcher NWT 150/750 + MII G2-8300

BR Sge

57967.39686

0.0004 I $\quad$ CCD + Clear $87 / 18 \quad$ Smolka M.

MNT $\quad 190 / 1000$

ATIK414EXM

DM Sge

57923.43736

0.0001 I CCD+Clear 229/163 Urbaník M.

Sky-Watcher NWT 150/750 + MII G2-8300 


\begin{tabular}{|c|c|c|c|c|c|}
\hline Star & & & & & \\
\hline HJD-2400000 & Error & I/II Method & Points & Observer & Technique \\
\hline 57965.42327 & 0.0003 & I $\quad \mathrm{CCD}+$ Clear & $181 / 69$ & Urbaník M. & $\begin{array}{l}\text { Sky-Watcher NWT 150/750 + } \\
\text { MII G2-8300 }\end{array}$ \\
\hline
\end{tabular}

AH Tau

57722.29992

57722.30010

57722.30029

57722.30038

57769.37343

57777.35800

BV Tau

58178.28495

CF Tau

58149.37262

58149.37301

CU Tau

57718.43374

58095.26191

58095.26220

NSVS 6733292 Tau

57718.39324

TY Tau

57781.27448

V0781 Tau

58182.63213

$\begin{array}{lllll}0.0001 & \text { I } & \text { CCD }+ \text { B } & 38 / 21 & \text { Lehky M. } \\ 0.0001 & \text { I } & \text { CCD }+\mathrm{V} & 36 / 20 & \text { Lehky M. } \\ 0.0001 & \text { I } & \text { CCD }+ \text { R } & 39 / 22 & \text { Lehky M. } \\ 0.0001 & \text { I } & \text { CCD }+ \text { I } & 36 / 22 & \text { Lehky M. } \\ 0.0001 & \text { II } & \text { CCD }+ \text { Clear } & 108 / 51 & \text { Banfi M. } \\ 0 & \text { II } & \text { CCD }+ \text { Clear } & 148 / 63 & \text { Banfi MV. }\end{array}$

0.0005 I CCD+Clear 207/73 Urbaník M.

\begin{tabular}{|c|c|c|}
\hline $0.0001 \mathrm{I}$ & $\mathrm{CCD}+\mathrm{R}$ & $130 / 58$ \\
\hline $0.0002 \mathrm{I}$ & $\mathrm{CCD}+\mathrm{V}$ & $127 / 57$ \\
\hline
\end{tabular}

0.0005 II CCD +Clear 283/233 Červinka L.

0.0002 I $\quad \mathrm{CCD}+\mathrm{V} \quad 237 / 66 \quad$ Šmelcer L.

$0.0002 \mathrm{I} \quad \mathrm{CCD}+\mathrm{R} \quad 228 / 66 \quad$ Šmelcer $\mathrm{L}$.

0.0012 I CCD + Clear $282 / 224$ Červinka L.

$\mathrm{R} 102 / 500+$ Atik 314L +

0.0003 II CCD +Clear 79/43 Mašek M.

NWT $150 / 600+$ CCD MII G2-1600 + comacorector

Sky-Watcher NWT 254/1200 + MII G2-402

Sky-Watcher NWT 254/1200 + MII G2-402

R102/500 + Atik 314L +

Sky-Watcher NWT 254/1200 + MII G2-402

Sky-Watcher NWT 254/1200 + MII G2-402 


\begin{abstract}
Star
HJD-2400000

57773.29902

57773.29903

V1234 Tau
\end{abstract}

58104.48136

58104.48217

58104.48247

V1237 Tau

58043.54266

58043.55183

58043.55323

V1241 Tau

58106.31784

V1260 Tau

57730.40512

V1370 Tau

57722.63743

57752.47936

57766.36947

57772.42215

58073.41039

58073.55635

WY Tau

57743.43974

58095.36235

$\begin{array}{lllrl}0.0007 & \mathrm{I} & \mathrm{CCD}+\mathrm{V} & 112 / 88 & \text { Mazanec J. } \\ 0.0005 \mathrm{I} & \mathrm{CCD}+\mathrm{R} & 122 / 96 & \text { Mazanec J. } \\ 0.0021 \text { I } & \mathrm{CCD}+\mathrm{I} & 114 / 89 & \text { Mazanec J. }\end{array}$

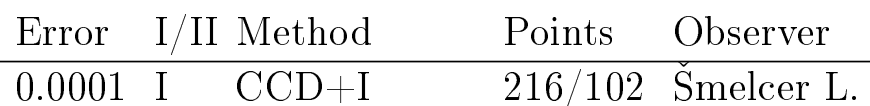

$0.0001 \mathrm{I} \quad \mathrm{CCD}+\mathrm{R} \quad 221 / 92 \quad \check{\text { Śmelcer } \mathrm{L} .}$

114/89 Mazanec J.
Technique

Celestron SCT 280/1765 +

MII G2-4000

Celestron SCT 280/1765

MII G2-4000

ORION, NWT 500/1500

MII G2 402

ORION, NWT 500/1500 +

MII G2 402

ORION, NWT 500/1500 MII G2 402

ORION, NWT 500/1500 + MII G2 402

ORION, NWT 500/1500 +

MII G2 402

ORION, NWT 500/1500 + MII G2 402

NWT 150/600 + CCD MII G2-1600 + comacorector

Sky-Watcher REF 80/520

MII G2-8300

0.0003 I CCD + Clear $\quad$ 160/91 Urbaník M.

0.0004 I CCD + Clear 392/283 Bragagnolo U. Sky-Watcher REF 100/900 + QHY9 0.0004 I $\quad$ CCD + Clear $41 / 18 \quad$ Urbaník M.

Sky-Watcher REF 80/520 + MII G2-8300

$300 \mathrm{~mm} \quad$ LX200 $\mathrm{SCT} / \mathrm{SBIG}$

$0.0003 \mathrm{I} \quad \mathrm{CCD}+\mathrm{V} \quad 87 / 45 \quad$ Gudmundsson

$\mathrm{S}$.

STL11k CCD

0.0003 II $\mathrm{CCD}+\mathrm{V} \quad 74 / 61 \quad$ Gudmundsson

$\mathrm{S}$.

$300 \mathrm{~mm}$ LX200 SCT/SBIG

STL11k CCD

205/37 Nosál P.

NWT 150/600 + Canon 20D + MPCC mk.III

205/127 Nosál' P. MPCC mk.III

HK25, NWT 250/1000

SBIG ST-7 + C

HK25, NWT 250/1000

SBIG ST-7 + Rc 
Star

HJD-2400000

Error I/II Method

Points

Observer

61/14 Mazanec J.

ORION, NWT 500/1500 MII G2 402

AB Tri

58085.38930

0.0024 I CCD + Clear 288/230 Vrašták M.

Pentacon 2,8/80+MII G2-1600

AK Tri

58027.56702

0.0005 II DSLR

92/25 Nosál' P.

NWT $150 / 600+$ Canon 20D + MPCC mk.III

AL Tri

58065.36862

58065.50200

0.0014 II DSLR

215/79 Nosál' P.

215/174 Nosál' P.

0.0018 I DSLR

BI Tri

58095.42509

58095.42580

58095.42590

58095.42626

BX Tri

57705.37614

57718.28255

57718.37654

57718.47448

57725.50386

57735.42729

57783.29395

57783.39134

58041.52343

58041.61807
0.0003 II $\mathrm{CCD}+\mathrm{R} \quad 162 / 125$ Mazanec J.

0.0004 II $\quad \mathrm{CCD}+\mathrm{V} \quad$ 156/118 Mazanec J.

0.0004 II $\quad$ CCD + I $\quad 161 / 124$ Mazanec J.

0.0005 II $\quad \mathrm{CCD}+\mathrm{B} \quad$ 158/121 Mazanec J.
Technique
NWT 150/600 + Canon 20D + MPCC mk.III

NWT 150/600 + Canon 20D + MPCC mk.III

ORION, NWT 500/1500 + MII G2 402

ORION, NWT 500/1500 MII G2 402

ORION, NWT 500/1500 + MII G2 402

ORION, NWT 500/1500 + MII G2 402

$300 \mathrm{~mm} \quad$ LX200 SCT/SBIG STL11k CCD

S.

$300 \mathrm{~mm}$ LX200 SCT/SBIG

STL11k CCD

S.

0.0004 II CCD + Clear 105/47 Gudmundsson

$\mathrm{S}$.

$300 \mathrm{~mm}$ LX200 SCT/SBIG

STL11k CCD

$300 \mathrm{~mm}$ LX200 SCT/SBIG

STL11k CCD

$\mathrm{S}$.

STN 254/1016+G2-8300

$300 \mathrm{~mm}$ LX200 SCT/SBIG

STL11k CCD

STN 254/1016+G2-8300

STN 254/1016+G2-8300

STN 254/1016+CCD-G2-8300

STN 254/1016+CCD-G2-8300 


\begin{tabular}{|c|c|c|c|c|c|}
\hline \multicolumn{6}{|l|}{ Star } \\
\hline HJD-2400000 & Error & I/II Method & Points & Observer & Technique \\
\hline 58101.24069 & 0.0001 & I $\quad \mathrm{CCD}+\mathrm{R}$ & $125 / 46$ & Šmelcer L. & $\begin{array}{l}\text { Sky-Watcher NWT 254/1200 } \\
+ \text { MII G2-402 }\end{array}$ \\
\hline 58101.24083 & 0.0002 & $\mathrm{CCD}+\mathrm{V}$ & $116 / 40$ & Šmelcer L. & $\begin{array}{l}\text { Sky-Watcher NWT 254/1200 } \\
\text { + MII G2-402 }\end{array}$ \\
\hline 58101.33584 & 0.0002 & $\mathrm{CCD}+\mathrm{R}$ & $125 / 112$ & Šmelcer L. & $\begin{array}{l}\text { Sky-Watcher NWT 254/1200 } \\
+ \text { MII G2-402 }\end{array}$ \\
\hline 58101.33647 & 0.0003 & $\mathrm{CCD}+\mathrm{V}$ & $116 / 99$ & Šmelcer L. & $\begin{array}{l}\text { Sky-Watcher NWT 254/1200 } \\
\text { + MII G2-402 }\end{array}$ \\
\hline 58113.27807 & 0.0004 & $\mathrm{CCD}+\mathrm{V}$ & $399 / 17$ & Šmelcer L. & $\begin{array}{l}\text { Sky-Watcher NWT 254/1200 } \\
\text { + MII G2-402 }\end{array}$ \\
\hline 58113.37680 & 0.0002 & $\mathrm{CCD}+\mathrm{V}$ & $399 / 150$ & Šmelcer L. & $\begin{array}{l}\text { Sky-Watcher NWT 254/1200 } \\
+ \text { MII G2-402 }\end{array}$ \\
\hline 58113.47133 & 0.0002 & $\mathrm{CCD}+\mathrm{V}$ & $399 / 279$ & Šmelcer L. & $\begin{array}{l}\text { Sky-Watcher NWT 254/1200 } \\
+ \text { MII G2-402 }\end{array}$ \\
\hline
\end{tabular}

BZ Tri

57716.32378

0.0003 I DSLR 231/68 Nosál P.

NWT 150/600 + Canon 20D + MPCC mk.III

CR Tri

58117.32636

$0.0001 \mathrm{I} \quad \mathrm{CCD}+\mathrm{R} \quad 65 / 15 \quad$ Mazanec J.

ORION, NWT 500/1500 MII G2 402

58117.32643

$0.0002 \mathrm{I} \quad \mathrm{CCD}+\mathrm{I} \quad 64 / 15 \quad$ Mazanec J.

ORION, NWT 500/1500 + MII G2 402

CSS J021749.2+331202 Tri

57661.37704

CzeV481 Tri

57661.55420

57725.45107

57783.32450

58027.59838

58041.55332

NSVS 6524869 Tri

57716.40909

0.0002 I $\quad C C D+$ Clear

0.0001 II $\mathrm{CCD}+$ Clear

392/284 Lomoz F.

299/76 Lomoz F.

244/107 Lomoz F.

NSVS 6551825 Tri

57716.26378

57716.50277
0.0008 I DSLR

87/48 Nosál' P.

0.0002 II CCD +Clear 495/246 Lomoz F.

0.0002 II $\mathrm{CCD}+$ Clear

0.0013 I DSLR

231/126 Nosál P.

228/29 Nosál P.

228/190 Nosál P.
STN 254/1016+G2-8300

Newton $300 / 1200+$ ST2000XM

STN 254/1016+G2-8300

SNT 254/1016+G2-8300

NWT 150/600 + Canon 20D + MPCC mk.III

STN 254/1016+CCD-G2-8300

NWT 150/600 + Canon 20D + MPCC mk.III

NWT 150/600 + Canon 20D + MPCC mk.III

NWT 150/600 + Canon 20D + MPCC mk.III 


\begin{tabular}{|c|c|c|c|c|c|c|}
\hline \multicolumn{7}{|l|}{ Star } \\
\hline HJD-2400000 & Error & $\mathrm{I} / \mathrm{I} \mathrm{l}$ & Method & Points & Observer & Technique \\
\hline 57707.25114 & 0.0003 & II & CCD + Clear & $444 / 162$ & Červinka L. & R102/500 + Atik 314L + \\
\hline 57774.26473 & 0.0001 & I & $\mathrm{CCD}+$ Clear & $114 / 37$ & Bragagnolo U. & $\begin{array}{l}\text { Sky-Watcher REF 100/900+ } \\
\text { QHY9 }\end{array}$ \\
\hline 58065.39687 & 0.0009 & II & DSLR & $216 / 100$ & Nosál' P. & $\begin{array}{l}\text { NWT } 150 / 600+\text { Canon 20D + } \\
\text { MPCC mk.III }\end{array}$ \\
\hline 58111.33924 & 0.0001 & I & $\mathrm{CCD}+\mathrm{V}$ & $232 / 86$ & Magris M. & $\begin{array}{l}\text { Achromatic refractor 120/600, } \\
\text { CCD Orion Starshoo }\end{array}$ \\
\hline
\end{tabular}

VSX J022549.6+294157 Tri

$\begin{array}{lllllll}57716.29554 & 0.001 & \text { I } & \text { DSLR } & 194 / 45 & \text { Nosál P. } & \begin{array}{l}\text { NWT 150/600 + Canon 20D + } \\ \text { MPCC mk.III }\end{array} \\ 57716.48706 & 0.001 & \text { II } & \text { DSLR } & 194 / 156 & \text { Nosál P. } & \begin{array}{l}\text { NWT 150/600 + Canon 20D + } \\ \text { MPCC mk.III }\end{array}\end{array}$

VZ Tri

58027.58275

0.0003 I DSLR $\quad 91 / 35 \quad$ Nosál P.

NWT 150/600 + Canon 20D + MPCC mk.III

WW Tri

58041.43200

58041.43201

0.0005 II CCD+Clear 179/88 Vrašták M.

NWT 240/1200+MII G2-1600, $80 / 400+$ G1-0300

0.0005 II CCD + Clear 179/88 Vrašták M.

NWT 240/1200+MII G2-1600, $80 / 400+$ G1-0300

X Tri

57737.27294

0.0001 I $\quad$ CCD + Clear $\quad 166 / 85 \quad$ Červinka L.

$\mathrm{R} 102 / 500+$ Atik $314 \mathrm{~L}+$

AA UMa

57721.57240

57847.50230

AW UMa

57881.64150

DW UMa

57840.28883

57840.42535

57840.56224
0.0001 I $\quad$ CCD + Clear $\quad 224 / 127$ Červinka L.

0.0008 I DSLR $\quad 189 / 140$ Sergey I.

0.0002 II Photometer + V59/26 Persha G.
R102/500 + Atik 314L +

Lens Jupiter-21 + Canon 400D

Meade RCT 250/2500 + Optec SSP-5a

HK25, NWT 250/1000

SBIG ST-7 + Rc

HK25, NWT 250/1000 +

SBIG ST-7 + Rc

HK25, NWT 250/1000 + SBIG ST-7 + Rc 


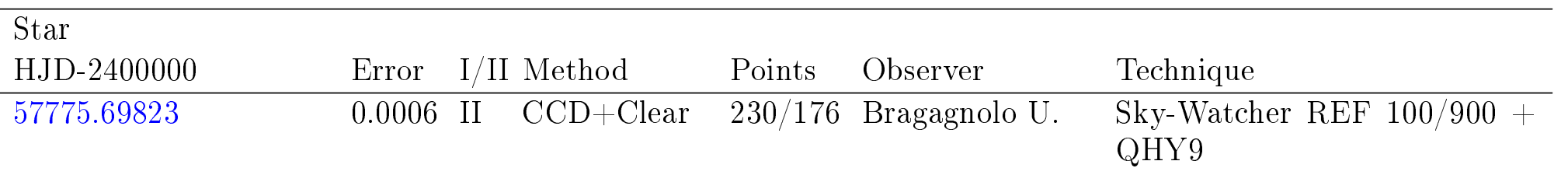

GZ UMa

58118.43774

HX UMa

57780.59249

II UMa

58197.71948

KM UMa

57839.32611

57839.32655

57839.32681

57839.32781

57839.50089

57839.50097

57839.50121

57839.50129

LINEAR 2945200

UMa

57780.66919

LP UMa

57840.32517

57840.48432
$0.0005 \mathrm{I} \quad \mathrm{CCD}+\mathrm{V} \quad 142 / 83 \quad$ Magris $\mathrm{M}$.

148/59 Nosál' P.

0.0019 I DSLR

NWT 150/600 + Canon 20D + MPCC mk.III

0.0007 II Photometer + V53/26 Persha G.

Meade RCT 250/2500 + Optec SSP-5a

$\mathrm{CCD}+0.5 \mathrm{x}$ reducer

$\begin{array}{lllll}0.0002 & \text { I } & \text { CCD }+\mathrm{I} & 116 / 16 & \text { Lehky M. } \\ 0.0002 \text { I } & \text { CCD }+ \text { R } & 113 / 15 & \text { Lehky M. } \\ 0.0002 & \text { I } & \text { CCD }+\mathrm{V} & 115 / 16 & \text { Lehky M. } \\ 0.0003 \text { I } & \text { CCD }+\mathrm{B} & 110 / 17 & \text { Lehky M. } \\ 0.0003 \text { II } & \text { CCD }+ \text { R } & 113 / 75 & \text { Lehky M. } \\ 0.0005 \text { II } & \text { CCD }+ \text { I } & 116 / 80 & \text { Lehky M. } \\ 0.0003 \text { II } & \text { CCD }+\mathrm{V} & 115 / 78 & \text { Lehky M. } \\ 0.0004 \text { II } & \text { CCD }+B & 110 / 76 & \text { Lehky M. }\end{array}$

JST, NWT 400/2000 + MII G2-1600 + BVRcIc

JST, NWT 400/2000 + MII G2-1600 + BVRcIc

JST, NWT 400/2000 + MII

G2-1600 + BVRcIc

JST, NWT 400/2000 + MII G2-1600 + BVRcIc

JST, NWT 400/2000 + MII

G2-1600 + BVRcIc

JST, NWT 400/2000 + MII G2-1600 + BVRcIc

JST, NWT 400/2000 + MII

G2-1600 + BVRcIc

JST, NWT 400/2000 + MII G2-1600 + BVRcIc

NWT $150 / 600+$ Canon 20D + MPCC mk.III

HK25, NWT 250/1000 + SBIG ST-7 + Rc

HK25, NWT 250/1000 + SBIG ST-7 + Rc 
Star

HJD-2400000

Error I/II Method

Points

Observer

167/78 Sergey I.

Lens Jupiter-21 + Canon 400D

57864.42813

0.0009 II DSLR

0.0011 I DSLR

165/99 Sergey I.

Lens Jupiter-21 + Canon 400D

57864.46459

NSVS 697648 UMa

57750.66345

57799.51360

57799.66881

NT UMa

57780.52595

NV UMa

57799.45170

OT UMa

57799.57158

$0.0002 \mathrm{I} \quad \mathrm{CCD}+$ Clear $\quad 178 / 67$ Lehky M.

0.0005 I CCD + Clear 228/115 Bragagnolo U.

0.0005 I CCD+Clear 408/126 Bragagnolo U.

0.0007 II CCD + Clear 408/337 Bragagnolo U.

0.0004 I CCD + Clear 86/44 Lehky M.

0.0003 II CCD + Clear 420/211 Bragagnolo U.

PZ UMa

57773.48074

57797.51730

57797.51813

57797.64724

57797.64740

57809.46611

57809.59953

57809.60020

57858.32272
$0.0001 \mathrm{I} \quad \mathrm{CCD}+$ Clear $32 / 16 \quad$ Walter F.

0.0002 II $\mathrm{CCD}+\mathrm{I}$

0.0002 II $\mathrm{CCD}+\mathrm{R}$

$0.0001 \mathrm{I} \quad \mathrm{CCD}+\mathrm{I}$

$0.0002 \mathrm{I} \quad \mathrm{CCD}+\mathrm{R}$

0.0002 I $\quad \mathrm{CCD}+$ Clear

0.0001 II $\quad \mathrm{CCD}+\mathrm{I}$

0.0001 II CCD + Clear 204/116 Walter F.

0.0002 I CCD + Clear $164 / 20$ Urbaník M.

173/78 Vrašták M

172/78 Vrašták M

173/157 Vrašták M

172/155 Vrašţák M

204/7 Walter F.

212/118 Walter F.
Sky-Watcher REF 100/900 + QHY9

Mertz, REF 160/1785 + SBIG ST-2000XM + C

HK25, NWT 250/1000 +

SBIG ST-7 + C

Sky-Watcher REF 100/900 + QHY9

Sky-Watcher REF 100/900 + QHY9

Sky-Watcher REF 100/900 + QHY9

MARK SCT 406/4060, SBIG ST10XME

NWT 280/1500+MII G2-1600, $80 / 400+$ G1-0300

NWT 280/1500+MII G2-1600, $80 / 400+$ G1-0300

NWT 280/1500+MII G2-1600, $80 / 400+$ G1-0300

NWT 280/1500+MII G2-1600, $80 / 400+$ G1-0300

MARK SCT 406/4060, SBIG ST10XME

MARK SCT 406/4060, SBIG ST10XME

MARK SCT 406/4060, SBIG ST10XME

Sky-Watcher NWT 150/750 + MII G2-8300 


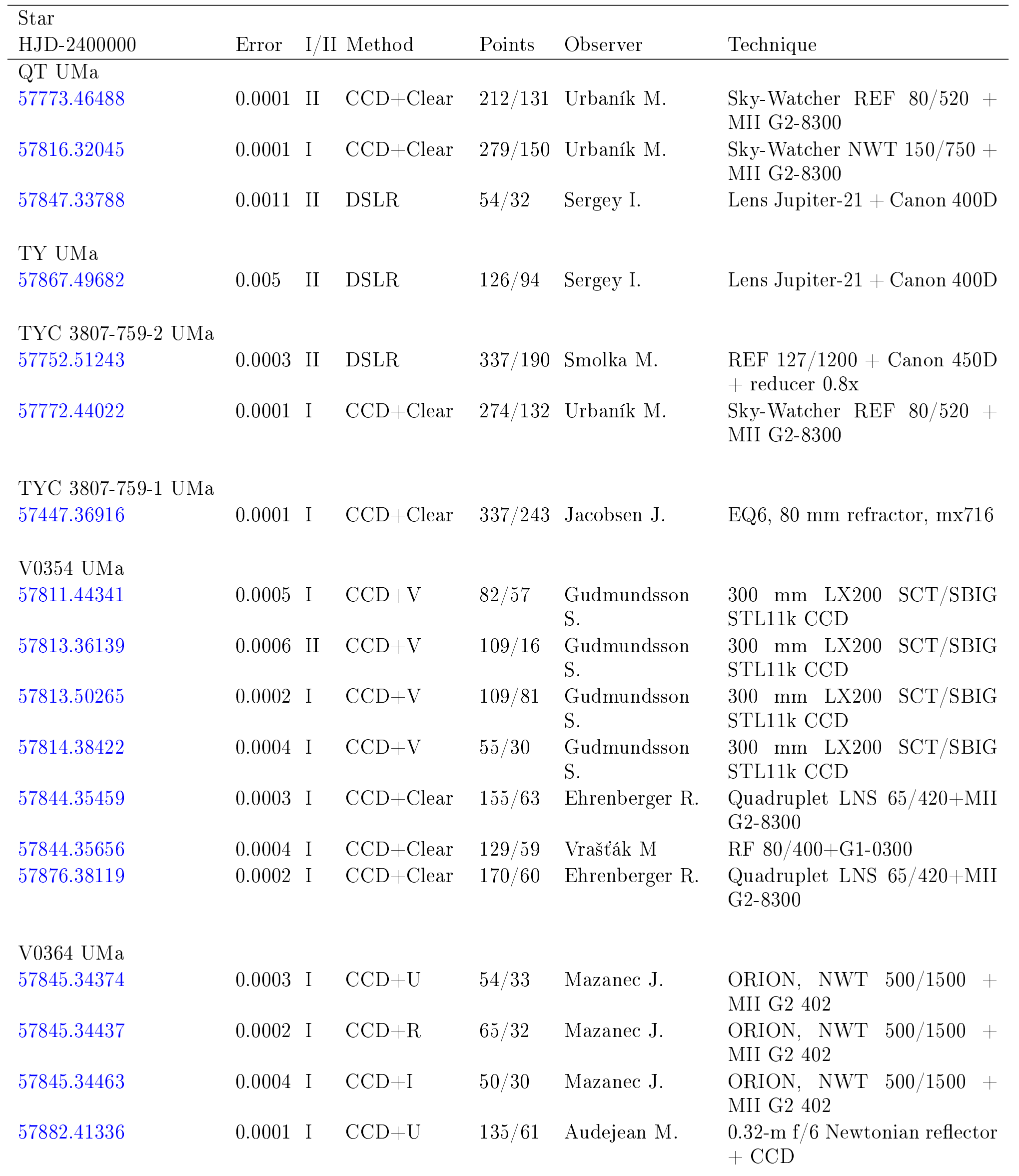




\begin{tabular}{|c|c|c|c|c|c|c|}
\hline $\begin{array}{l}\text { Star } \\
\text { HJD-2400000 }\end{array}$ & Error & & Method & Points & Observer & Technique \\
\hline VSX J094636. & UMa & & & & & \\
\hline 57848.51498 & 0.0016 & I & DSLR & $196 / 145$ & Sergey I. & Lens Jupiter-21 + Canon 400D \\
\hline VV UMa & & & & & & \\
\hline 57773.61688 & 0.0001 & I & CCD + Clear & $390 / 190$ & Bragagnolo U. & $\begin{array}{l}\text { Sky-Watcher REF } 100 / 900+ \\
\text { QHY9 }\end{array}$ \\
\hline 57799.39405 & 0.0007 & II & $\mathrm{CCD}+$ Clear & $273 / 156$ & Červinka L. & $\mathrm{R} 102 / 500+$ Atik $314 \mathrm{~L}+$ \\
\hline 58118.67963 & 0.0003 & I & $\mathrm{CCD}+\mathrm{V}$ & $132 / 67$ & Magris M. & $\begin{array}{l}\text { REF } 120 / 600+\text { Orion } 3 \mathrm{G} \\
\mathrm{CCD}+0.5 \mathrm{x} \text { reducer }\end{array}$ \\
\hline W UMa & & & & & & \\
\hline 54844.61896 & 0.0003 & I & $\mathrm{CCD}+\mathrm{V}$ & $53 / 32$ & $\begin{array}{l}\text { Gudmundsson } \\
\text { S. }\end{array}$ & $\begin{array}{l}300 \mathrm{~mm} \text { LX200 SCT/ssp-3 } \\
\text { Photometer }\end{array}$ \\
\hline 54865.47352 & 0.0002 & II & $\mathrm{CCD}+\mathrm{V}$ & $88 / 34$ & $\begin{array}{l}\text { Gudmundsson } \\
\text { S. }\end{array}$ & $\begin{array}{l}300 \mathrm{~mm} \text { LX200 SCT/ssp-3 } \\
\text { Photometer }\end{array}$ \\
\hline 54872.47935 & 0.0002 & II & $\mathrm{CCD}+\mathrm{V}$ & $68 / 33$ & $\begin{array}{l}\text { Gudmundsson } \\
\text { S. }\end{array}$ & $\begin{array}{l}300 \mathrm{~mm} \text { LX200 SCT/ssp-3 } \\
\text { Photometer }\end{array}$ \\
\hline 54873.48243 & 0.0005 & II & $\mathrm{CCD}+\mathrm{V}$ & $38 / 23$ & $\begin{array}{l}\text { Gudmundsson } \\
\text { S. }\end{array}$ & $\begin{array}{l}300 \mathrm{~mm} \text { LX200 SCT/SBIG } \\
\text { STL11k CCD }\end{array}$ \\
\hline 57704.35694 & 0.0002 & II & $\mathrm{CCD}+\mathrm{V}$ & $1201 / 544$ & 4 Jacobsen J. & $\begin{array}{l}\text { EQ6,102 } \mathrm{mm} \text { refractor, Atik } \\
314\end{array}$ \\
\hline 57706.35875 & 0.0002 & II & $\mathrm{CCD}+\mathrm{V}$ & $454 / 327$ & Jacobsen J. & $\begin{array}{l}\text { EQ6,102 mm refractor, Atik } \\
314\end{array}$ \\
\hline 57841.31177 & 0.0001 & $\mathrm{I}$ & CCD+Clear & $253 / 33$ & Vrašták M & RF 80/400+G1-0301 \\
\hline 58180.61527 & 0.0001 & I & Photometer + I & $\mathrm{V} 39 / 17$ & Persha G. & $\begin{array}{l}\text { Meade RCT 250/2500 + Optec } \\
\text { SSP-5a }\end{array}$ \\
\hline
\end{tabular}

XY Uma 58112.48970 $0.0001 \mathrm{I} \quad \mathrm{CCD}+\mathrm{V} \quad 214 / 105 \quad$ Magris M. Achromatic refractor 120/600, CCD Orion Starshoo

ZZ UMa 57824.34269 0.0001 I CCD + Clear 297/167 Červinka L. R102/500 + Atik 314L +

RT UMi 58173.29682
0.0009 I DSLR $\quad 136 / 31 \quad$ Sergey I.
Newton $200 \mathrm{~mm}(1: 5)+$ Canon 400D
Kometensucher Zeiss $200 / 1370+$ SBIG ST7 


\begin{tabular}{|c|c|c|c|c|c|c|}
\hline \multirow{3}{*}{$\begin{array}{l}\text { Star } \\
\text { HJD-2400000 } \\
57776.47911\end{array}$} & \multirow{3}{*}{$\begin{array}{l}\text { Error } \\
0.0003\end{array}$} & \multirow{2}{*}{\multicolumn{2}{|c|}{ I/II Method }} & \multirow{3}{*}{$\begin{array}{l}\text { Points } \\
201 / 34\end{array}$} & \multirow{2}{*}{$\begin{array}{l}\text { Observer } \\
\text { Walter F }\end{array}$} & \multirow[b]{2}{*}{ Technique } \\
\hline & & & & & & \\
\hline & & I & $\mathrm{CCD}+$ Clear & & Walter F. & $\begin{array}{ll}\text { Kometensucher Zeiss } & \text { REF } \\
200 / 1370+\text { SBIG ST7 } & \end{array}$ \\
\hline 57776.47914 & 0.0005 & I & DSLR & $357 / 66$ & Walter F. & $\begin{array}{l}\text { CZ Mirotar SCT 180/1000, } \\
\text { Canon 350d }\end{array}$ \\
\hline 57917.52982 & 0.0004 & II & $\mathrm{CCD}+$ Clear & $177 / 115$ & Bragagnolo U. & $\begin{array}{l}\text { Sky-Watcher REF 100/900 + } \\
\text { QHY9 }\end{array}$ \\
\hline 58080.58177 & 0.0003 & I & DSLR & $331 / 181$ & $\begin{array}{l}\text { Walter F., Začal } \\
\text { M. }\end{array}$ & $\begin{array}{l}\text { Zeiss MCT } 350 / 3300+\text { Canon } \\
\text { 350d }\end{array}$ \\
\hline
\end{tabular}

WW UMi

57392.30956

0.0008 I CCD +Clear 229/118 Jacobsen J.

EQ6, $100 \mathrm{~mm}$ refractor, Atik 57841.36374

0.0008 I $\quad$ CCD + Clear $\quad 107 / 42 \quad$ Ehrenberger R. 314

Quadruplet LNS 65/420+MII G2-8300

ASAS J081131-4634.7 Vel

$\begin{array}{llllll}58085.70818 & 0.0007 \text { I } & \text { CCD }+ \text { R } & 87 / 45 & \text { Mašek M. } & \begin{array}{l}\text { FRAM, Nikkor LNS 106/300 } \\ + \text { G4-16000 }\end{array} \\ 58125.73067 & 0.0007 \text { I } & \text { CCD }+ \text { R } & 69 / 51 & \text { Mašek M. } & \begin{array}{l}\text { FRAM, Nikkor LNS 106/300 } \\ + \text { G4-16000 }\end{array}\end{array}$

ASAS J082030-4326.7 Vel

57748.67657

0.0002 I $\mathrm{CCD}+\mathrm{R}$

133/49 Mašek M.

FRAM, Nikkor LNS 106/300

58085.70624

0.0003 I $\quad \mathrm{CCD}+\mathrm{R} \quad 87 / 44 \quad$ Mašek M.

+ G4-16000

FRAM, Nikkor LNS 106/300

+ G4-16000

ASAS J083036-4556.9 Vel

58085.66109

$0.0004 \mathrm{I} \quad \mathrm{CCD}+\mathrm{R} \quad$ 86/19 Mašek M.

FRAM, Nikkor LNS 106/300 + G4- 16000

ASAS J084015-4607.2 Vel

$\begin{array}{llllll}57750.74718 & 0.0004 \text { I } & \text { CCD }+ \text { R } & 39 / 31 & \text { Mašek M. } & \begin{array}{l}\text { FRAM, Nikkor LNS 106/300 } \\ + \text { G4-16000 }\end{array} \\ 58085.74545 & 0.0001 \text { I } & \text { CCD }+ \text { Clear } & 87 / 67 & \text { Mašek M. } & \begin{array}{l}\text { FRAM, Nikkor LNS 106/300 } \\ + \text { G4-16000 }\end{array} \\ 58125.72512 & 0.0003 \text { I } & \text { CCD }+ \text { R } & 67 / 46 & \text { Mašek M. } & \begin{array}{l}\text { FRAM, Nikkor LNS 106/300 } \\ + \text { G4-16000 }\end{array}\end{array}$

AY Vel

57748.73019

0.0006 I $\quad C C D+R \quad$ 141/106 Mašek M.

FRAM, Nikkor LNS 106/300

+ G4-16000 


\begin{tabular}{|c|c|c|c|c|c|}
\hline Star & & & & & \\
\hline HJD-2400000 & Error & I/II Method & Points & Observer & Technique \\
\hline 57748.71805 & 0.0001 & I $\quad \mathrm{CCD}+\mathrm{R}$ & $120 / 85$ & Mašek M. & $\begin{array}{l}\text { FRAM, Nikkor LNS } 106 / 300 \\
+ \text { G4-16000 }\end{array}$ \\
\hline
\end{tabular}

BP Vel

58085.66421

58125.67598

0.0002 I $\quad C C D+$ Clear $87 / 20 \quad$ Mašek M.

0.0002 I $\quad \mathrm{CCD}+\mathrm{R} \quad 69 / 19 \quad$ Mašek M.

BU Vel

58085.74508

0.0005 II CCD +Clear 87/67 Mašek M.

BW Vel

57750.68975

0.0005 I $\quad \mathrm{CCD}+\mathrm{R}$

25/11 Mašek M.

EQ Vel

57750.76532

$0.0005 \mathrm{I} \quad \mathrm{CCD}+\mathrm{R} \quad 36 / 30 \quad$ Mašek M.

58085.65041

$0.0004 \mathrm{I} \quad \mathrm{CCD}+\mathrm{R}$

87/13 Mašek M.

GDS J0845421-461151 Vel

57750.72699

$0.0014 \mathrm{I} \quad \mathrm{CCD}+\mathrm{R} \quad 38 / 27 \quad$ Mašek M.

GDS J0851081-443915 Vel

57750.67271

0.0016 I $\quad \mathrm{CCD}+\mathrm{R} \quad 31 / 6 \quad$ Mašek M.

NSV 4126 Vel

58085.74228

58125.69589

$0.0001 \mathrm{I} \quad \mathrm{CCD}+\mathrm{R}$

0.0002 I $\quad \mathrm{CCD}+\mathrm{R}$

69/31 Mašek M.

87/65 Mašek M.

AH Vir

57800.67857

$0.0001 \mathrm{I} \quad \mathrm{CCD}+\mathrm{B} \quad 87 / 55 \quad$ Mašek M.

ASAS J115055+0615.6 Vir

58149.61506

0.0006 I DSLR

100/60 Nosál P.
FRAM, Nikkor LNS 106/300

+ G4-16000

FRAM, Nikkor LNS 106/300 + G4-16000

FRAM, Nikkor LNS 106/300

+ G4-16000

NWT 150/600 + CCD MII

G2-1600 + comacorector

FRAM, Nikkor LNS 106/300 + G4-16000

FRAM, Nikkor LNS 106/300 + G4-16000

FRAM, Nikkor LNS 106/300

FRAM, Nikkor LNS 106/300

+ G4-16000

FRAM, Nikkor LNS 106/300 FRAM, Nikkor LNS 106/300 + G4-16000 


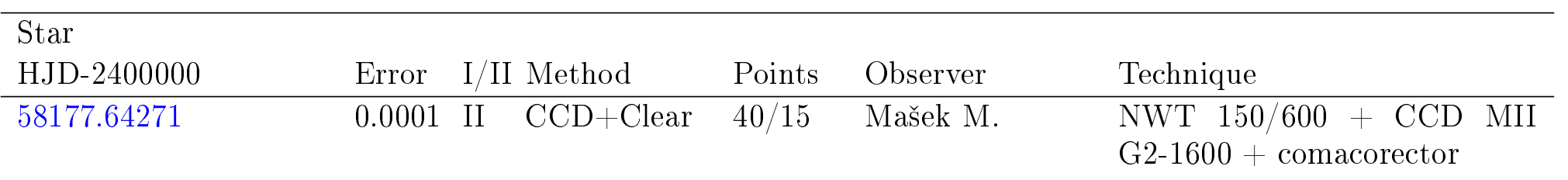

BF Vir

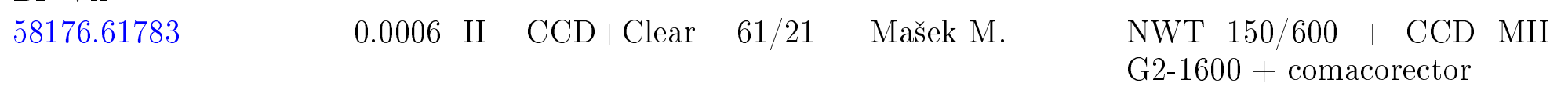

CSS J130429.3-082421 Vir

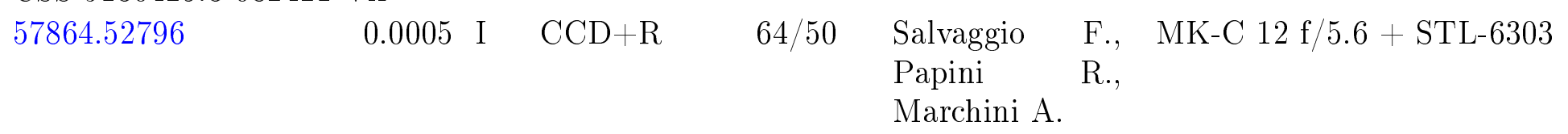

CSS J130508.9-085339 Vir

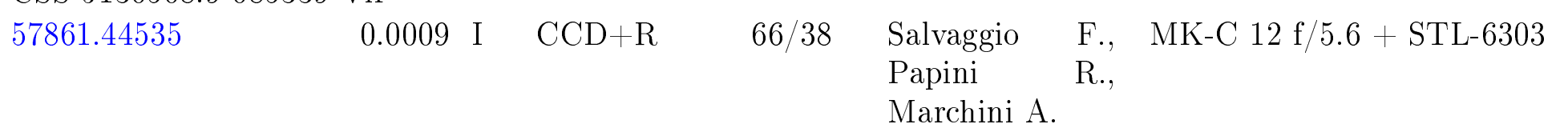

CSS J130518.0-093455 Vir

$\begin{array}{lllll}57857.49396 & 0.0006 \text { I } & \mathrm{CCD}+\mathrm{R} \quad 60 / 41 & \begin{array}{l}\text { Salvaggio } \\ \text { Marchini } \\ \end{array} & \text { F., } \\ & \text { Papini R. }\end{array}$

CSS J130557.4-124840 Vir

$\begin{array}{rlllll}57837.60282 & 0.001 & \text { I } & \text { CCD }+ \text { Clear } 40 / 31 & \begin{array}{l}\text { F. Salvaggio, } \\ \text { A. Marchini, R. }\end{array} & \text { STL-6303 } 12 \mathrm{f} / 5.6+\mathrm{GM}-2000+ \\ & & & \end{array}$
Papini

HW Vir

57841.43130

57841.49007

57841.54836

57878.37364

58186.51305

IK Vir

57814.55484

0.0012 I DSLR

0.0002 II $\quad \mathrm{CCD}+\mathrm{R}$

$0 \quad \mathrm{I} \quad \mathrm{CCD}+\mathrm{R}$

0.0002 II $\mathrm{CCD}+\mathrm{R}$

$0 \quad \mathrm{I} \quad \mathrm{CCD}+\mathrm{R}$

$0 \quad$ I $\quad \mathrm{CCD}+$ Clear

215/59 Šmelcer L.

215/106 Šmelcer L.

215/173 Šmelcer L.

90/73 ̌melcer L.

245/33 ̌̌melcer L.
Celestron SCT 355/2460 + MII G2-1600

Celestron SCT 355/2460 + MII G2-1600

Celestron SCT 355/2460 + MII G2-1600

Celestron SCT 355/2460 + MII G2-1600

Celestron SCT 280/1765 + MII G2-4000

NWT 150/600 + Canon 20D + MPCC mk.III

PS Vir

57799.60653

0.0001 I $\quad C C D+$ Clear $\quad 92 / 59 \quad$ Mašek M.

NWT $150 / 600+$ CCD MII 


\begin{tabular}{|c|c|c|c|c|c|}
\hline \multicolumn{6}{|l|}{ Star } \\
\hline HJD-2400000 & Error & I/II Method & Points & Observer & Technique \\
\hline 58173.59884 & 0.0001 & II $\quad$ CCD + Clear & $66 / 18$ & Mašek M. & $\begin{array}{l}\text { NWT } 150 / 600+\text { CCD MII } \\
\text { G2-1600 + comacorector }\end{array}$ \\
\hline 58174.61490 & 0.0001 & CCD + Clear & $96 / 49$ & Mašek M. & $\begin{array}{l}\text { NWT } 150 / 600+\text { CCD MII } \\
\text { G2-1600 + comacorector }\end{array}$ \\
\hline
\end{tabular}

PY Vir

\begin{tabular}{|c|c|c|c|c|c|}
\hline 58172.54479 & $0.0003 \mathrm{II}$ & $\mathrm{CCD}+$ Clear & $107 / 14$ & Mašek M. & $\begin{array}{l}\text { NWT } 150 / 600+\text { CCD MII } \\
\text { G2-1600 + comacorector }\end{array}$ \\
\hline \multicolumn{6}{|l|}{ V0610 Vir } \\
\hline 57811.65312 & $0.0001 \mathrm{I}$ & $\mathrm{CCD}+$ Clear & $276 / 230$ & Lomoz F. & STN 254/1016+G2-8300 \\
\hline 57840.37415 & 0.0002 II & $\mathrm{CCD}+$ Clear & $251 / 126$ & Lomoz F. & STN 254/1016+G2-8300 \\
\hline 57840.54204 & $0.0002 \mathrm{I}$ & $\mathrm{CCD}+$ Clear & $288 / 177$ & $\begin{array}{l}\text { Walter F., Hla- } \\
\text { dík B. }\end{array}$ & $\begin{array}{l}\text { REF } 200 / 1370+\text { SBIG ST7 + } \\
\text { MuniWin }\end{array}$ \\
\hline 58166.48211 & $0.0001 \mathrm{I}$ & CCD + Clear & $212 / 94$ & Lomoz F. & Newton $300 / 1200+$ ST2000XM \\
\hline
\end{tabular}

ASAS J201418+2704.8 Vul

58026.35280

0.0002 I $\quad \mathrm{CCD}+\mathrm{R} \quad 89 / 67 \quad$ Mašek M.

NWT 150/600 + CCD MII G2-1600 + comacorector

BK Vul

57994.52273

0.0004 I CCD + Clear $153 / 44$ Červinka L.

R102/500 + Atik 320E mono, $0.5 \mathrm{x} \mathrm{FC}$

BT Vul

57969.41427

0.0003 I $\quad$ CCD + Clear $\quad 32 / 8$

Walter F., Lya-

$\mathrm{CZ}$ Kometensucher

REF chová K., Hla- 200/1370 + SBIG ST7 dík B.

58085.24388

0.0003 II CCD+Clear 158/95 Bragagnolo U.

Sky-Watcher REF 100/900 + QHY9

BU Vul

57739.22750

$0.0001 \mathrm{I} \quad \mathrm{CCD}+\mathrm{R} \quad 110 / 47 \quad$ Šmelcer L.

Celestron SCT 280/1765 +

57739.22795

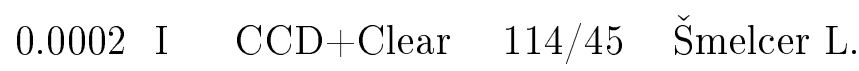

MII G2-4000

Celestron SCT 280/1765 +

MII G2-4000

CD Vul

58073.27742

$0.0001 \mathrm{I} \quad \mathrm{CCD}+\mathrm{R} \quad 321 / 161$ Šmelcer L.

Celestron SCT 355/2460 + MII G2-1600

EU Vul 


\begin{tabular}{|c|c|c|c|c|c|c|}
\hline $\begin{array}{l}\text { Star } \\
\text { HJD-2400000 }\end{array}$ & Error & $\mathrm{I} / \mathrm{II}$ & Method & Points & Observer & Technique \\
\hline GP Vul & & & & & & \\
\hline 58042.30616 & 0.0001 & $\mathrm{I}$ & $\mathrm{CCD}+\mathrm{R}$ & $332 / 130$ & Šmelcer L. & $\begin{array}{l}\text { Sky-Watcher NWT 254/1200 } \\
+ \text { MII G2-402 }\end{array}$ \\
\hline IM Vul & & & & & & \\
\hline 57714.29093 & 0.0002 & II & $\mathrm{CCD}+\mathrm{R}$ & $423 / 236$ & Šmelcer L. & $\begin{array}{l}\text { Celestron SCT } 280 / 1765+ \\
\text { MII G2-4000 }\end{array}$ \\
\hline UCAC4 $588-123574$ & & & & & & \\
\hline Vul & & & & & & \\
\hline 57994.50571 & 0.0006 & $\mathrm{I}$ & CCD + Clear & $148 / 23$ & Červinka L. & $\begin{array}{l}\mathrm{R} 102 / 500+\text { Atik } 320 \mathrm{E} \text { mono, } \\
0.5 \mathrm{x} \text { FC }\end{array}$ \\
\hline V0392 Vul & & & & & & \\
\hline 57956.43331 & 0.0003 & $\mathrm{I}$ & CCD + Clear & $364 / 178$ & $\begin{array}{l}\text { Souza de Joode } \\
\text { M., Lamberská } \\
\text { I. }\end{array}$ & NWT 150/750 + SBIG ST7 \\
\hline
\end{tabular}


Table 2: List of observers and corresponding amount of obtained times of minima.

\begin{tabular}{|c|c|c|}
\hline$\#$ & Observer(s) name(s) & Minima times total \\
\hline 1 & Lehký M. & 294 \\
\hline 2 & Bílek F. & 241 \\
\hline 3 & Šmelcer L. & 233 \\
\hline 4 & Mašek M. & 164 \\
\hline 5 & Urbaník M. & 157 \\
\hline 6 & Walter F. & 130 \\
\hline 7 & Bragagnolo U. & 117 \\
\hline 8 & Nosál’ P. & 114 \\
\hline 9 & Červinka L. & 93 \\
\hline 10 & Mazanec J. & 92 \\
\hline 11 & Vrašt’ák M. & 81 \\
\hline 12 & Lomoz F. & 72 \\
\hline 13 & Hanžl D. & 47 \\
\hline 14 & Sergey I. & 32 \\
\hline 15 & Gudmundsson S. & 30 \\
\hline 16 & Jacobsen J. & 26 \\
\hline 17 & Ehrenberger R. & 24 \\
\hline 18 & Hladík B. & 24 \\
\hline 19 & Magris M. & 19 \\
\hline 20 & Tylšar M. & 19 \\
\hline 21 & Persha G. & 19 \\
\hline 22 & Školník V. & 15 \\
\hline 23 & Smolka M. & 13 \\
\hline 24 & Audejean M. & 12 \\
\hline 25 & Trnka J. & 12 \\
\hline 26 & Medulka T. & 10 \\
\hline 27 & Šuchaň J. & 8 \\
\hline 28 & Salvaggio F. & 7 \\
\hline 29 & Papini R. & 7 \\
\hline 30 & Marchini A. & 6 \\
\hline 31 & Colaco C. & 6 \\
\hline 32 & Vala J. & 6 \\
\hline 33 & Starck M. & 5 \\
\hline 34 & Quiňones C. & 5 \\
\hline 35 & Auer R. F. & 4 \\
\hline 36 & Melia R. & 4 \\
\hline 37 & Ruocco N. & 4 \\
\hline
\end{tabular}


Tabulka 2 - continued from previous page

\begin{tabular}{llc}
\hline$\#$ & Observer & Minima times total \\
\hline 38 & Kalášek J. & 4 \\
39 & Jíra S. & 4 \\
40 & Banfi M. & 4 \\
41 & Girardici C. & 2 \\
42 & Tornatore M. & 2 \\
43 & Boková S., & 2 \\
44 & Malinak J. & 2 \\
45 & Novotný P. & 2 \\
46 & Mokrý A. & 2 \\
47 & Banfi M. & 2 \\
48 & Castillo M. & 1 \\
49 & Durantini L. & 1 \\
50 & Hrádek L. & 1 \\
51 & Kubica T. & 1 \\
52 & Lamberská I. & 1 \\
53 & López O. & 1 \\
54 & Lyachová K. & 1 \\
55 & Mrňák P. & 1 \\
56 & Pavlíková E. & 1 \\
57 & Souza de Joode M. & 1 \\
58 & Vilchis E. & 1 \\
59 & Začal M. & 1 \\
& & \\
\hline
\end{tabular}

\section{Acknowledgements}

We are grateful to all the observers for their contributions, and we would like to give special thanks to Václav Přibík for technical support of the server var.astro.cz and Anton Paschke for maintaining the $\mathrm{O}-\mathrm{C}$ gateway which was used to get light ephemeris of observed stars. This research was made using the SIMBAD database, maintained by CDS, Strasbourg, France. We often used the International Variable Star Index (VSX) database, operated at AAVSO, Cambridge, Massachusetts. We would like to thank the Pierre Auger Collaboration for the use of its facilities. The operation of the robotic telescope FRAM is supported by the EU grant GLORIA (No. 283783 in FP7-Capacities program) and by the grant of the Ministry of Education of the Czech Republic (MSMTCR LM2015038). The data calibration and analysis related to FRAM telescope is supported by the Ministry of Education of the Czech Republic MSMT-CR (LG15014 and

\footnotetext{
${ }^{\dagger}$ Referred to in Table 1 as CCD Group in Úpice Observatory
} 
CZ.02.1.01/0.0/0.0/16_013/0001402).

\section{Reference}

Brát, L., Mikulášek, Z., Pejcha, O., 2012, Minima Timing of eclipsing binaries, http://var2.astro.cz/library/1350745528_ebfit.pdf

Chrastina, M., Mikulášek, Z., Zejda, M., 2014, CoSka, 43, 422, 2014CoSka..43..422C

Hroch, F., 1998, Proc. 29th conf. Variable Star Research, held 7. - 9. November 1997, Brno, Czech Republic, ed. Jiří Dušek and Miloslav Zejda p. 30, 1998stel.conf...30H

Mikulášek, Z., 2015, A\&A, 584, 8, 2015A\&A...584A...8M

Motl, D., 2007, C-Munipack, http://c-munipack.sourceforge.net/

Stetson, P. B., 1991, DAOPHOT II: Next Generation. MIDAS User Manual

Stetson, P. B., 1987, PASP, 99, 191, 1987PASP...99..191S

Pojmanski, G., 2005, ASAS-3, http://www.astrouw.edu.pl/asas/?page=main 